\title{
Phospholamban - \\ Identification of novel interaction partners
}

\author{
Dissertation \\ for the award of the degree \\ "Doctor rerum naturalium" \\ of the Georg-August-Universität Göttingen \\ within the doctoral program Molecular medicine \\ of the Georg-August University School of Science (GAUSS)
}

Submitted by

Daniel Kownatzki-Danger (né Kownatzki)

Born in Hamburg, Germany

Göttingen, January 2020 



\section{Thesis Committee}

Prof. Dr. Stephan E. Lehnart (Supervisor)

Clinic for Cardiology and Pneumology, University Medical Center, Göttingen

Prof. Dr. Peter Rehling

Department of Cellular Biochemistry, Göttingen

Prof. Dr. Blanche Schwappach-Pignataro

Department of Molecular Biology, University Medical Center, Göttingen

\section{Members of the Examination Board}

Referee:

Prof. Dr. Stephan E. Lehnart (Supervisor)

Clinic for Cardiology and Pneumology, University Medical Center, Göttingen

$2^{\text {nd }}$ Referee:

Prof. Dr. Peter Rehling

Department of Cellular Biochemistry, Göttingen

\section{Further members of the Examination Board}

Prof. Dr. Dörthe M. Katschinski

Institute of Cardiovascular Physiology, University Medical Center, Göttingen

Prof. Dr. Henning Urlaub

Bioanalytical Mass Spectrometry, Max Planck Institute for biophysical Chemistry, Göttingen

Prof. Dr. Ralph Kehlenbach

Department of Molecular Biology, University Medical Center, Göttingen

Date of oral examination: $5^{\text {th }}$ June 2020 

To my family 



\section{Contents}

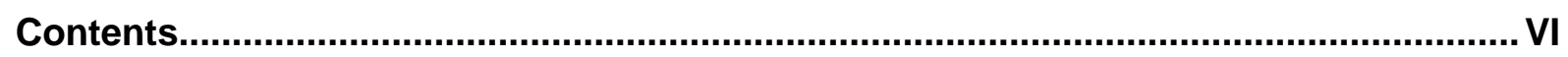

List of Publications ..............................................................................................

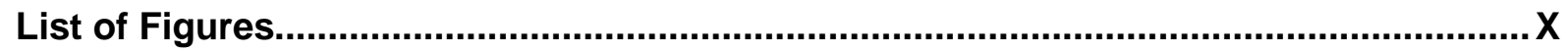

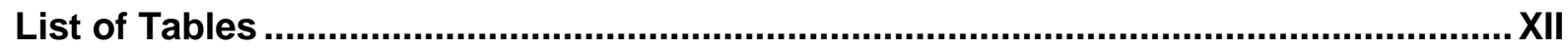

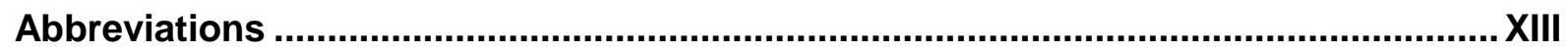

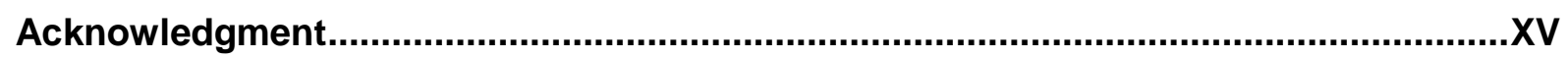

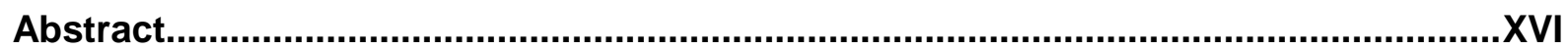

1 Introduction .................................................................................................... 1

1.1 SERCA2a: Sarco/endoplasmic reticulum $\mathrm{Ca}^{+2}$ ATPase 2a.............................. 1

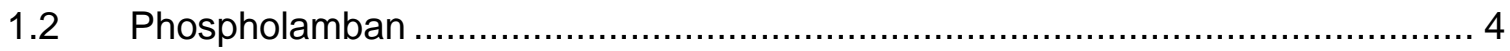

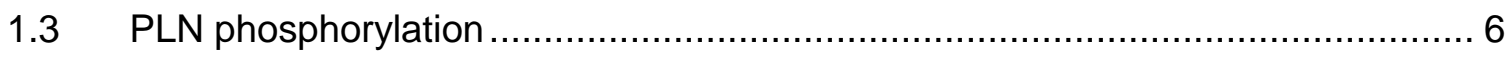

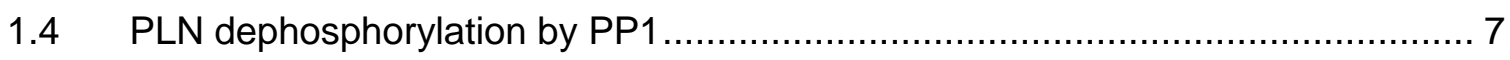

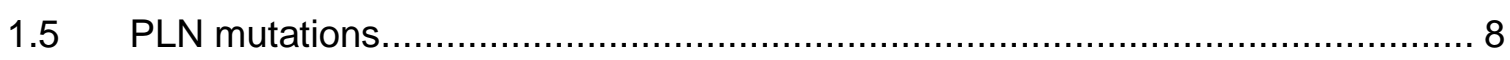

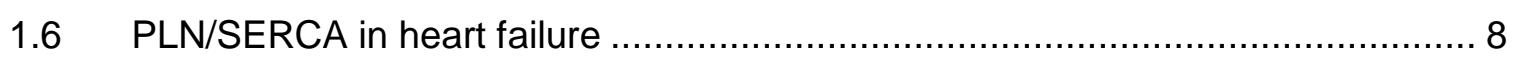

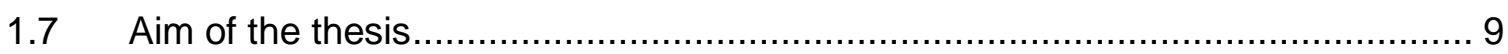

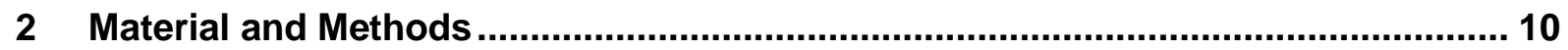

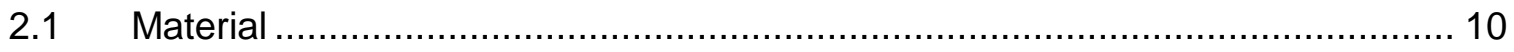

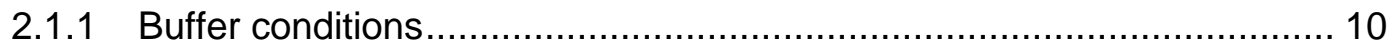

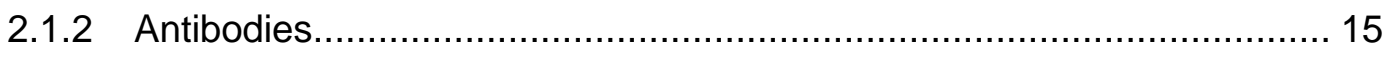

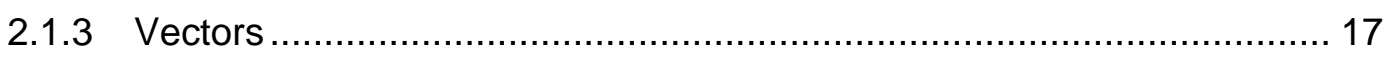

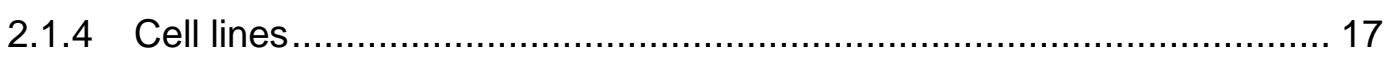

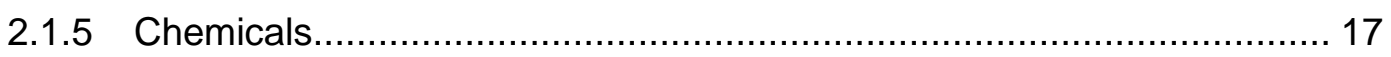

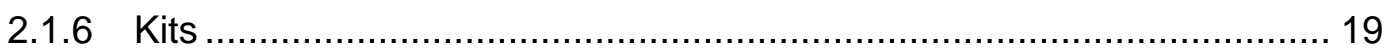

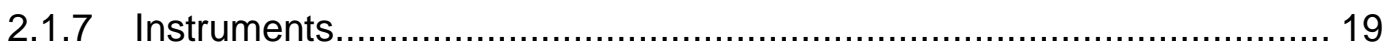

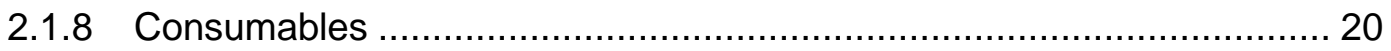

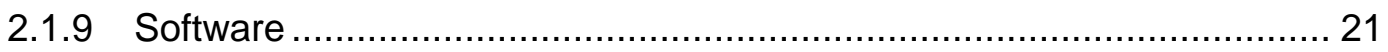

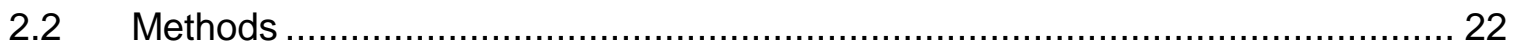

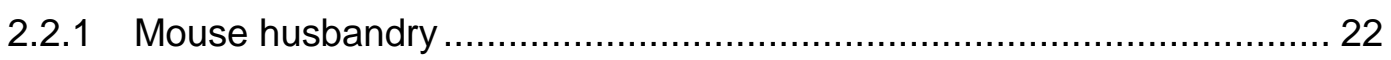

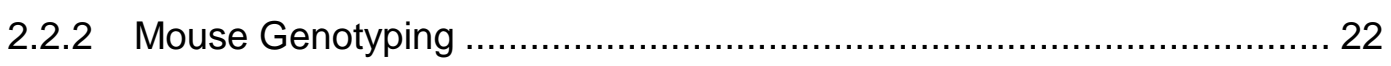

2.2.3 Cloning of V5-APEX2-PLN and V5-APEX2-PLNA(1-29) ...................... 23 
2.2.4 Adenoviral constructs for transduction ............................................ 24

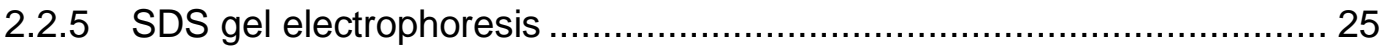

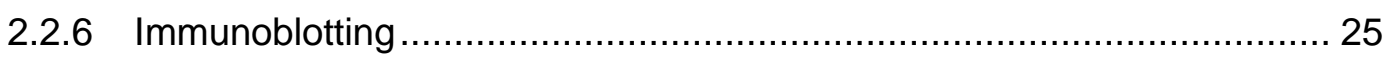

2.2.7 Co-Immunoprecipitation of phospholamban interacting proteins ............ 25

2.2.8 Cell cultivation of HEK293A ............................................................ 26

2.2.9 Isolation of neonatal rat cardiomyocytes ........................................... 26

2.2.10 NRCM cultivation in SILAC medium ............................................ 27

2.2.11 Human induced pluripotent stem cell differentiation ........................... 28

2.2.12 Transfection of HEK293A cells and NRCM ...................................... 28

2.2.13 Adenoviral transduction of NRCMs ................................................. 29

2.2.14 APEX2 Proximity Labeling ............................................................... 29

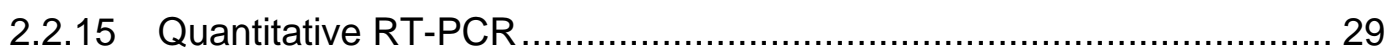

2.2.16 Cell lysis, enrichment and elution of biotinylated proteins ................... 30

2.2.17 Transverse aortic constriction (TAC) to generate pressure overload in mice 31

2.2.18 Isolation of adult mouse ventricular cardiomyocytes ......................... 31

2.2.19 Sample preparation of mouse heart tissue for biochemistry ................ 32

2.2.20 Sample preparation for confocal and STED immunofluorescence imaging 32

2.2.21 Histology of ventricular heart tissue ............................................... 33

2.2.22 Confocal microscopy ............................................................. 34

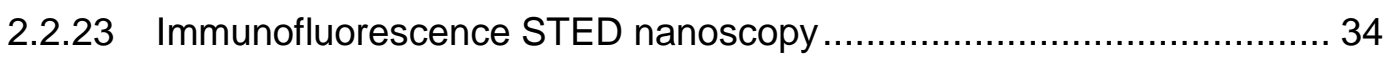

2.2.24 Membrane enrichment from isolated ventricular cardiomyocytes......... 34

2.2.25 Membrane solubilization for blue native gel electrophoresis ............... 35

2.2.26 Blue native gel electrophoresis ..................................................... 35

2.2 .27 2D BNE/SDS-PAGE .................................................................... 36

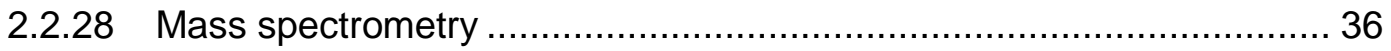

2.2.29 APEX2 proximity labeling and nanoLC-MS/MS analysis for quantitative mass spectrometry .............................................................................. 36

2.2.30 APEX2 proximity labeling data processing ................................. 37

2.2.31 Gene enrichment analysis .................................................... 38

2.2.32 Complexome profiling and nanoLC-MS/MS analysis ........................ 38

2.2.33 Complexome profiling data processing ......................................... 38

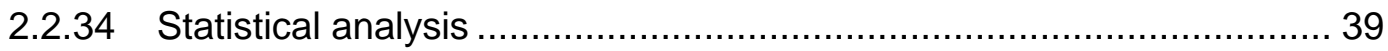

3 Identification of novel phospholamban protein interactions by complexome profiling. 


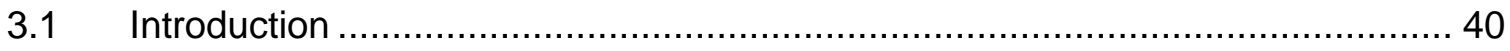

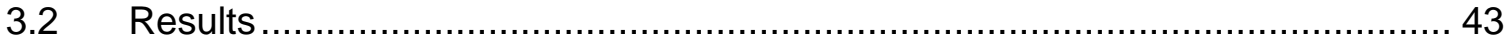

3.2.1 Isolation and solubilization of cardiac membranes for BN-PAGE .......... 43

3.2.2 Complexome profiling analysis of native cardiac membranes ............... 47

3.2.3 SLMAP forms high molecular weight complexes ............................... 55

3.2.4 SLMAP localization and PLN proximity in mouse ventricular

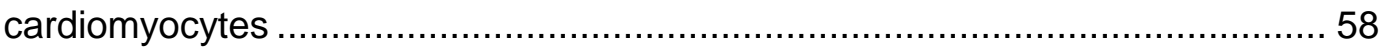

3.2.5 SLMAP expression is altered in PLN KO mouse hearts and cardiac

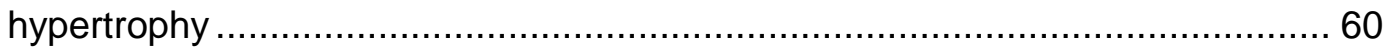

3.2.6 SLMAP in human iPSC-derived cardiomyocytes ................................ 63

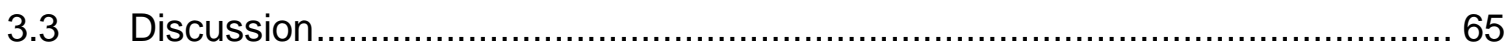

3.3.1 Isolation and solubilization of cardiac membranes for BN-PAGE .......... 65

3.3.2 Complexome Profiling analysis of native cardiac membranes................66 66

3.3.3 SLMAP proximity to PLN in cardiomyocytes ......................................68

3.3.4 SLMAP expression changes in cardiac hypertrophy .............................68

3.3.5 SLMAP expression in human iPSC-derived cardiomyocytes ................ 69

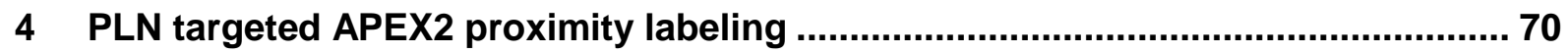

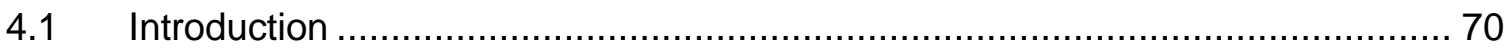

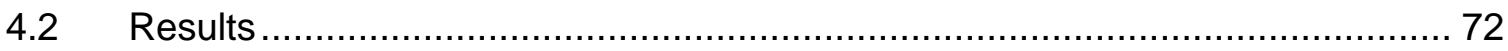

4.2.1 APEX2-PLN proximity labeling in HEK293A cells .............................. 72

4.2.2 Neonatal rat cardiomyocyte cell model .......................................... 75

4.2.3 Ratiometric proximity proteomics in NRCMs .................................... 78

4.2.4 Adenoviral transduction for proximity labeling in NRCMs ...................... 79

4.2.5 PLN specific ratiometric APEX2 proximity labeling in NRCMs .............. 82

4.2.6 Analysis of ratiometric PLN targeted APEX2 proteomic data ................. 85

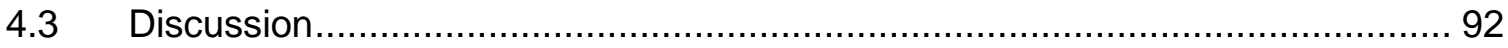

4.3.1 PLN targeted APEX2 proximity labeling in HEK293A and NRCM ......... 92

4.3.2 APEX2-PLN targeting in neonatal rat cardiomyocytes ......................... 93

4.3.3 Quantitative proteomic analysis of NRCMs ...................................... 93

4.3.4 Analysis of ratiometric PLN targeted APEX2 proteomic data ................ 93

4.3.5 Gene enrichment analysis of APEX2-PLN enriched proteins ................. 94

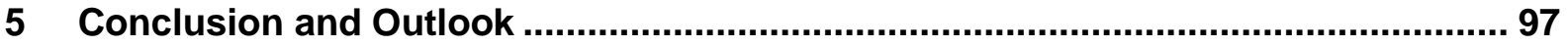

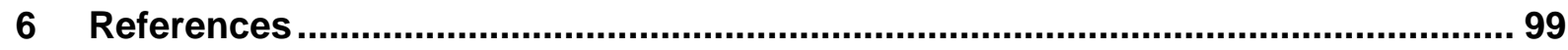

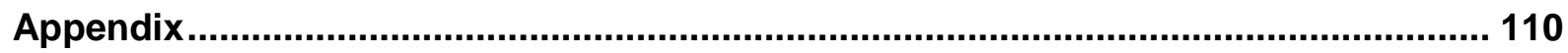




\section{List of Publications}

Alsina KM, Hulsurkar M, Brandenburg S, Kownatzki-Danger D, Lenz C, Urlaub H, Abu-Taha I, Kamler M, Chiang DY, Lahiri SK, Reynolds JO, Quick AP, Scott L Jr, Word TA, Gelves MD, Heck AJR, Li N, Dobrev D, Lehnart SE, Wehrens XHT. Loss of Protein Phosphatase 1 Regulatory Subunit PPP1R3A Promotes Atrial Fibrillation. Circulation. 2019;140:681-693. DOI: 10.1161/CIRCULATIONAHA.119.039642

Publications not included in thesis

Brandenburg S, Pawlowitz J, Fakuade FE, Kownatzki-Danger D, Kohl T, Mitronova GY, Scardigli M, Neef J, Schmidt C, Wiedmann F, Pavone FS, Sacconi L, Kutschka I, Sossalla S, Moser T, Voigt N and Lehnart SE (2018) Axial Tubule Junctions Activate Atrial $\mathrm{Ca}^{2+}$ Release Across Species. Front. Physiol. 9:1227. doi: 10.3389/fphys.2018.01227 


\section{List of Figures}

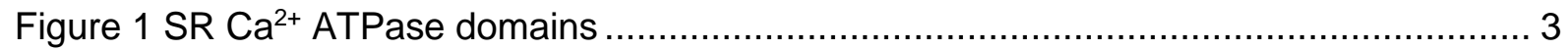

Figure 2 Scheme of the E1/E2 model for SERCA ............................................................. 4

Figure 3 PLN sequence homology for various species ............................................. 5

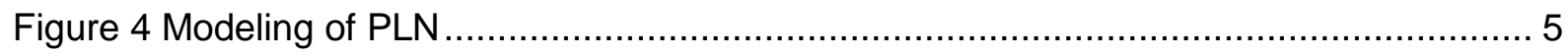

Figure 5 Adenoviral vector constructs for transduction of NRCMs .................................. 24

Figure 6 Complexome profiling workflow for cardiac membranes .................................... 41

Figure 7 Topology of the sarcolemmal membrane-associated protein SLMAP1-3 isoforms. 42

Figure 8 Membrane isolation from mouse ventricular cardiomyocytes ............................... 44

Figure 9 Membrane isolation and solubilization from ventricular cardiomyocytes ............... 46

Figure 10 BN-PAGE gel for complexome profiling and mass calibration........................... 48

Figure 11 Complexome profiling of OXPHOS complexes .............................................. 50

Figure 12 Complexome profiling of mouse ventricular cardiomyocyte membranes .............. 51

Figure 13 Complexome profiling of kinase and phosphatase distributions ......................... 53

Figure 14 Complexome profiling shows novel 'SR Ca ${ }^{2+}$ cycling complex' ........................... 55

Figure 15 SLMAP isoforms form high molecular weight complexes .................................. 57

Figure 16 SLMAP shows a transversal striated pattern in mouse ventricular tissue............. 59

Figure 17 SLMAP is co-purified with PLN by affinity purification ..................................... 60

Figure 18 Mouse ventricular tissue expression of SLMAP in WT and PLN KO mouse hearts

Figure 19 SLMAP protein expression is increased in mouse ventricular tissue after TAC.... 62 Figure 20 SLMAP protein expression is unchanged in PLN KO ventricular tissue after TAC 63 Figure 21 SLMAP is expressed in human iPSC-derived cardiomyocytes.

Figure 22 Proximity proteomics of canine phospholamban for the identification of novel interaction partners

Figure 23 Confocal co-immunofluorescence imaging of APEX2-PLN and APEX2-PLNA(1-29) in HEK293A cells

Figure 24 PLN based proximity labeling in transfected HEK293A cells 73

Figure 25 Affinity purification optimization of biotinylated proteins after proximity labeling in HEK293A

Figure 26 Confocal co-immunofluorescence imaging of endogenous PLN and SERCA2a in NRCMs 76

Figure 27 Confocal co-immunofluorescence imaging of APEX2-PLN and APEX2-PLN $\triangle(1-29)$ in NRCMs.

Figure 28 PLN specific proximity labeling in plasmid transfected NRCMs. 78 
Figure 29 Stable isotope labeling of amino acids in cell culture (SILAC) incorporation in NRCMs

Figure 30 Analysis of adenoviral particle concentrations for NRCM transduction. 80

Figure 31 Confocal co-immunofluorescence imaging of adenoviral APEX2-PLN and APEX2PLN $\Delta(1-29)$ expression in NRCMs 81

Figure 32 PLN targeted APEX2 proximity labeling in NRCMs for ratiometric proteomics ..... 82

Figure 33 Ratiometric SILAC APEX2 proximity proteomics in NRCMs 84

Figure 34 Analysis of ratiometric PLN targeted APEX2 proteomic data 86

Figure 35 Protein enrichment after APEX2-PLN proximity labeling in NRCMs. 88

Figure 36 Gene enrichment analysis of APEX2-PLN enriched proteins. 90 


\section{List of Tables}

Table 1 Distribution of SERCA isoforms in mammalian tissue ............................................ 2

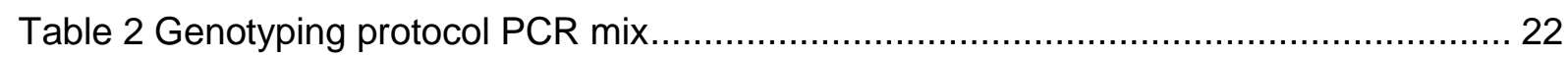

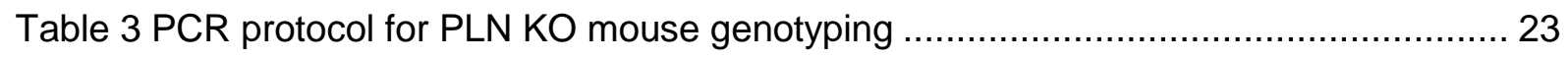

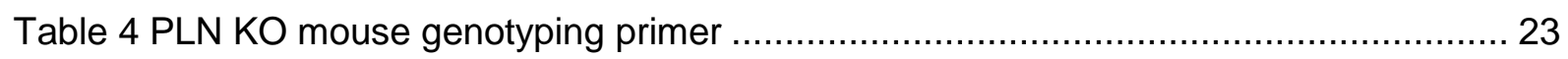

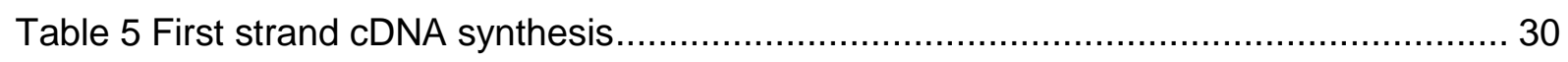

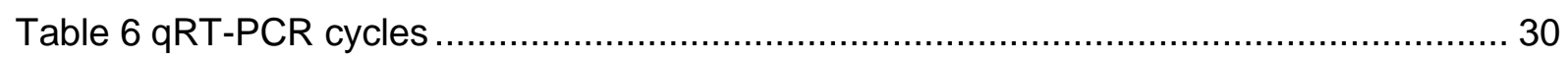

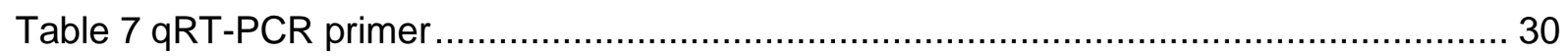

Table 8 Deparaffinization and rehydration of paraffin embedded histological sections......... 34

Table 9 APEX2-PLN enriched proteins after PLN targeted proximity labeling................... 110

Table 10 Gene family enriched proteins after PLN targeted APEX2 proximity labeling in NRCM 


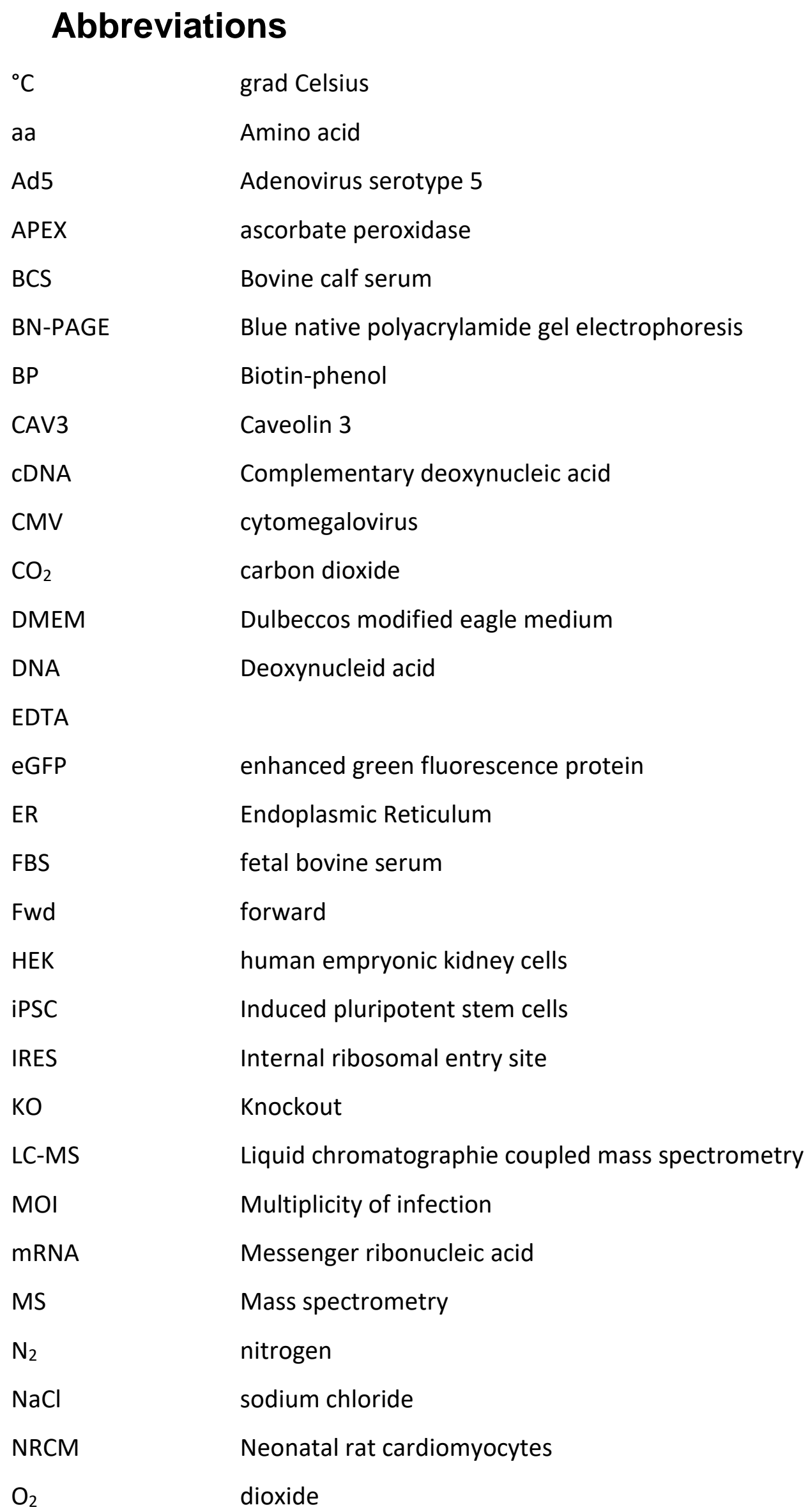




$\begin{array}{ll}\text { OXPHOS } & \text { Oxidate phosphorylation } \\ \text { PBS } & \text { Phosphate buffer saline } \\ \text { PCR } & \text { Polymerase chain reaction } \\ \text { Pen/Strep } & \text { penicillin/streptomycin } \\ \text { PFA } & \text { Paraformaldehyde } \\ \text { PLM } & \text { phospholemman } \\ \text { PLN } & \text { Phospholamban } \\ \text { qRT-PCR } & \text { Quantitative real-time polymerase chain reaction } \\ \text { Rev } & \text { reverse } \\ \text { RT } & \text { room temperature } \\ \text { RyR2 } & \text { Ryanodine receptor 2 } \\ \text { SeC } & \text { second } \\ \text { SERCA } & \text { Sarcoendoplasmic reticulum Calcium ATPase } \\ \text { SILAC } & \text { Stable isotop labeling by amino acids in cell culture } \\ \text { SLMAP } & \text { Sarcolemmal membrane-associated protein } \\ \text { SR } & \text { Sarcoplasmic reticulum } \\ \text { STED } & \text { Stimulated Emission Depletion } \\ \text { TAC } & \text { transvers aortic constriction } \\ \text { VCM } & \text { Ventricular cardiomyocytes }\end{array}$




\section{Acknowledgment}

I would like to express my gratitude to my supervisor Prof. Dr. Stephan E. Lehnart, for giving me the opportunity to do my Ph.D. in his group - for his guidance and support during this time. I have learned a lot throughout my time, and I am thankful for this experience.

I wish to thank my thesis advisory committee members Prof. Dr. Blanche Schwappach and Prof. Dr. Peter Rehling, for their helpful and interesting discussions and giving inputs from a different perspective. Especially, I would like to thank Prof. Dr. Blanche Schwappach for giving me additional scientific input and for the great collaboration throughout the last years.

In addition, I want to thank Prof. Dr. Dörthe Katschinski, Prof. Dr. Henning Urlaub, and Prof. Dr. Ralph Kehlenbach, for joining my extended examination board.

I want to thank all my co-workers from the Lehnart group. I truly had a great time working in the lab. Thank you for the great support over the last years. Moreover, thanks to all for the helpful discussions, all interesting ideas, and critical thinking concerning my experiments. Many thanks to Brigitte, Birgit, and Christiane for your support and technical assistance in and outside the lab.

Special thanks to the Proteomic Service Unit, Dr. Christof Lenz, Prof. Dr. Henning Urlaub, Lisa Neuenroth, and Thierry Wasselin, for your great support, helpful discussions, and setting up complexome profiling, which made up for a big part of my thesis.

Great thanks to Dr. Lukas Cyganek and the Stem Cell Unit for your help, support, and providing stem cells for my thesis.

I especially thank Eva, Jonas, Dennis, and Sören for creating a genuinely nice and happy environment over the last years. I really enjoyed it!

I am extremely grateful to my friends 'Bergsteiger' and 'Familien-Kommune'.

I am very grateful to my parents, parents-in-law, and my family, for your constant loving support, always encouraging me and being there for me.

Finally, and most importantly, I am extremely grateful to Katharina for loving me and supporting me throughout the past years, whether in Kiel, Kranenburg, or Göttingen.

Thank you, Henri Johann, for being there and bringing so much joy in our lives. 


\section{Abstract}

Cardiac contraction and relaxation depend on intracellular $\mathrm{Ca}^{2+}$ release and sequestration in the sarcoplasmic reticulum (SR). The small tail-anchored protein phospholamban (PLN) is essential for normal stress-adaptation of heart function. The dephosphorylated PLN form binds to the $\mathrm{Ca}^{2+}$ ATPase SERCA2a and potentially to other important SER proteins and thereby regulates the reuptake of $\mathrm{Ca}^{2+}$ into the SR. To elucidate the PLN associated signalosome in endogenous SR domains, we developed a PLN-specific proximity assay and applied complexome profiling on native cardiac membranes.

Here, a mass spectrometry-based method described as complexome profiling, originally established to identify OXPHOS protein supercomplexes and assembly factors, is used to elucidate the composition of essential protein complexes important for the intracellular $\mathrm{Ca}^{2+}$ cycling in ventricular cardiomyocytes. Digitonin solubilized, enriched membrane fractions of isolated ventricular cardiomyocytes from wildtype and phospholamban knockout mice were loaded and separated by blue native gradient gel electrophoresis (BN-PAGE). Gel lanes were cut, and trypsin digested followed by mass spectrometry (LC-MS/MS).

Hierarchical clustering and analysis of migration patterns confirmed distinct groups of comigrating proteins, most prominently OXPHOS complexes, which were used for quantitative validation. Importantly, at higher molecular weight, a novel SR $\mathrm{Ca}^{2+}$ cycling complex comprised of the RyR2 calcium release channel, SERCA2a, and each regulatory protein subunits were identified. Furthermore, the sarcolemmal membrane-associated protein (SLMAP) was identified as a potential interacting protein of PLN.

Combined with phospholamban knockout, complexome profiling enabled the close-to-native analysis of previously unknown macromolecular protein complexes comprised of dual RyR2SERCA2a transporters, changes in abundance due to phospholamban deficiency.

Additionally, for proteomic mapping, APEX2, a genetically engineered peroxidase, was fused $\mathrm{N}$-terminally to generate APEX2-PLN. Adenoviral expression and biotin-phenol treatment were used to label proteins in nanometric proximity of PLN by biotinylation in living neonatal rat cardiomyocytes (NRCMs). APEX2-biotinylated proteins were enriched by pulldown, processed, and analyzed by mass spectrometry (LC-MS/MS). Additionally, for ratiometric analysis, NRCMs were cultivated with stable isotope labeled amino acids (SILAC). A truncated APEX2-PLN $\Delta$ (1-29) construct was used as SR-targeted control and enhanced green fluorescent protein (eGFP) as a negative control.

APEX2-PLN expression in NRCMs was confirmed by Western blot (WB) and fluorescence microscopy through the bicistronically expressed eGFP. Confocal analysis showed that 
APEX2-PLN co-localizes with endogenous SERCA2a similar to endogenous PLN. The medium and heavy labeled amino acids (SILAC) were incorporated at a rate of $>95 \%$, thus enabling global quantitative proteomic analysis. 14-3-3 proteins were identified as significantly enriched gene family for APEX2-PLN biotinylation.

A PLN-specific strategy for proximity labeling was successfully developed in live NRCM and verified for known interaction partners. Proteomic proximity analysis identified previously unknown PLN protein-protein interactions in the neonatal heart. Furthermore, these potential interactors will be exploited in the adult heart, i.e. by combining the proximity data with highresolution microscopy. 


\section{Introduction}

Calcium $\left(\mathrm{Ca}^{2+}\right)$ is a universal second messenger and involved in diverse cellular processes and signaling pathways ${ }^{1}$. In the heart, $\mathrm{Ca}^{2+}$ plays a central role in cardiomyocyte excitation, contraction, and relaxation ${ }^{2}$. An increase of the cytosolic $\mathrm{Ca}^{2+}$ concentration leads to the activation of the contraction apparatus, whereas its deceleration initiates the relaxation phase. Upon depolarization of the plasma membrane, $\mathrm{Ca}^{2+}$ enters the cytosol through L-type $\mathrm{Ca}^{2+}$ channels (dihydropyridine receptors; DHPRs). The increased local $\mathrm{Ca}^{2+}$ concentration stimulates the opening of $\mathrm{Ca}^{2+}$ release channels (Ryanodine receptors; $\mathrm{RyR}$ ) and the release of $\mathrm{Ca}^{2+}$ from the sarcoplasmic reticulum (SR). The increased cytosolic $\mathrm{Ca}^{2+}$ concentration leads to troponin $\mathrm{C}$ binding and the activation of muscle contraction. The relaxation phase is initiated by the removal of $\mathrm{Ca}^{2+}$ from the cytosol by plasma-membrane $\mathrm{Ca}^{2+}$-ATPases (PMCAs) or $\mathrm{Na}^{+} / \mathrm{Ca}^{2+}$ exchangers (NCXs), which are located in the plasma membrane, or by the sarco/endoplasmic reticulum $\mathrm{Ca}^{2+}$-ATPase $2 \mathrm{a}$ (SERCA2a), located in the SR. More than $70 \%$ of the $\mathrm{Ca}^{2+}$ removal from the cytosol is through SERCA2a. Its activity is determined by the regulatory protein phospholamban (PLN), which in turn is regulated through the B-adrenergic signaling pathway. Dephosphorylated PLN inhibits SERCA2a activity, while its phosphorylation by PKA or CamK2 releases the inhibitory effect. Hence, SERCA2a determines not only the rate of $\mathrm{Ca}^{2+}$ removal but also the size of the $\mathrm{Ca}^{2+}$ store in the SR increasing the contractility proportional ${ }^{3}$. This makes SERCA2a a key protein and regulator of muscle contractility.

\subsection{SERCA2a: Sarco/endoplasmic reticulum $\mathrm{Ca}^{+2}$ ATPase 2a}

SERCA belongs similar to the Na,K-ATPase and the H,K-ATPase to the family of P-type ATPases, which are highly conserved in structure ${ }^{4}$. The SERCA family includes several isoforms, which are encoded by three homologous genes SERCA1, SERCA2, and SERCA3 $(\text { Table } 1)^{5}$. The gene of SERCA1 encodes for two isoforms by alternative splicing, SERCA1a and SERCA1b. They represent the adult and fetal isoform in fast-twitch skeletal muscle, respectively6. The SERCA2 gene encodes for a transcript that gives rise to three different isoforms by alternative splicing: SERCA2a, SERCA2b, and SERCA2c. SERCA2a and SERCA2b are mainly expressed in slow-twitch skeletal muscle cells and cardiac muscle cells, while SERCA2c has been detected in epithelial, mesenchymal and hematopoietic cell lines ${ }^{7,6}$ as well as in cardiac muscle ${ }^{8}$. SERCA3 gives at protein level rise to isoform $3 a, 3 b$, and $3 c$ and is present in a large variety of non-muscle cell types, especially in the hematopoietic cell lineages ${ }^{9,10}$. 
Table 1 Distribution of SERCA isoforms in mammalian tissue

\begin{tabular}{|c|c|c|c|c|c|c|c|c|}
\hline \multirow[b]{3}{*}{ SERCA isoform } & \multicolumn{4}{|c|}{ Skeletal muscle } & & & & \\
\hline & \multicolumn{2}{|c|}{ Fast twitch } & \multicolumn{2}{|c|}{ Slow twitch } & \multicolumn{2}{|c|}{ Cardiac muscle } & \multirow{2}{*}{$\begin{array}{l}\text { Smooth } \\
\text { muscle }\end{array}$} & \multirow{2}{*}{$\begin{array}{c}\text { Non-muscle } \\
\text { cells }\end{array}$} \\
\hline & Fetal & Adult & Fetal & Adult & Fetal & Adult & & \\
\hline SERCA1a & - & +++++ & - & - & - & - & - & - \\
\hline SERCA1b & +++ & - & - & - & - & - & - & - \\
\hline SERCA2a & + & - & + & +++ & + & ++++ & + & - \\
\hline SERCA2b & + & + & + & + & + & + & + & + \\
\hline SERCA2C & & & & & & + & & + \\
\hline SERCA3a & - & - & - & - & - & - & - & + \\
\hline SERCA3b & - & - & - & - & - & - & - & + \\
\hline SERCA3C & - & - & - & - & - & - & - & + \\
\hline
\end{tabular}

Based on the crystal structure of SERCA1a, $\mathrm{Ca}^{2+}$ transport is well described ${ }^{11,12,13}$. Recently the first crystal structure of SERCA2a has been resolved, showing high similarity to SERCA1a, which indicates a conserved mechanism ${ }^{14}$. In general, SERCA is composed of three cytoplasmic domains, namely the nucleotide binding domain $(\mathrm{N})$, the phosphorylation domain $(P)$, the actuator domain $(A)$, and one transmembrane domain (M) (Figure 1).

The N-domain contains the binding site for adenosine triphosphate (ATP). In SERCA1 and SERCA2, but not in SERCA3, an interaction site for the reversible inhibitor phospholamban is located in the N-domain. The P-domain is the functional core of the SR $\mathrm{Ca}^{2+}$-ATPase and contains the sequence motive DKTGTLT in which $D$ is the reversibly phosphorylated asparagine (Asp351). This sequence is conserved in all P-type ATPases ${ }^{15}$. The A-domain facilitates the conformational change for $\mathrm{Ca}^{2+}$ transport. The $\mathrm{M}$-domain is composed of ten membrane-spanning helices (M1-M10) and is the least conserved domain amongst P-type ATPases with a sequence homology of $18 \%$. It contains the $\mathrm{Ca}^{2+}$ translocation site, which is coordinated by the polar and ionic side chains of the transmembrane helices M4, M5, M6, and $\mathrm{M} 8$ and allows the localization of two bound $\mathrm{Ca}^{2+}$ ions ${ }^{16}$. They are centrally located and surrounded on the one side by M1-3 on the opposite side by M8-10. M6 and M7 are separated by a long cytosolic loop, which mediates the interaction of the $\mathrm{P}$ and $\mathrm{M}$-domain and forms another interaction site of phospholamban. 


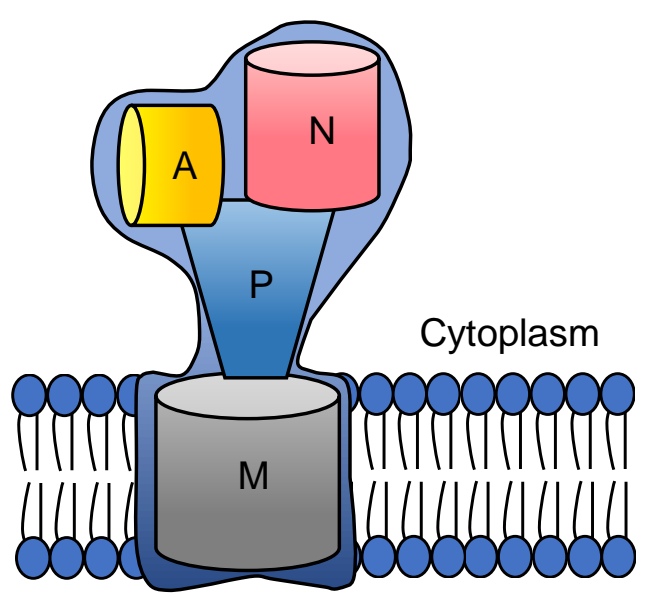

SR lumen

\section{Figure $1 \mathrm{SR} \mathrm{Ca}^{2+}$ ATPase domains}

The SR $\mathrm{Ca}^{2+}$ ATPase consists of three cytosolic and one transmembrane domain. The nucleotide binding $(\mathrm{N})$-domain is shown in red, the phosphorylation $(\mathrm{P})$-domain in blue, the actuator $(\mathrm{A})$-domain in yellow, and the membrane (M)-domain in grey. Through the binding of two $\mathrm{Ca}^{2+}$ ions within the $\mathrm{M}$ domain and ATP binding to the N-domain, the resulting phosphorylation of Asp351 in the P-domain initiates a conformational change and the release of $\mathrm{Ca}^{2+}$ into the SR lumen.

The reaction of ATP-binding, hydrolysis and phosphorylation of the P-domain and the energy transfer to the ion binding site is mostly the same in all P-type ATPases ${ }^{17}$. The extrusion of $\mathrm{Ca}^{2+}$ from the cytosol is facilitated by SERCA and initiates the muscle relaxation phase. The mechanistic transport of two $\mathrm{Ca}^{2+}$ ions per cycle is described at different conformational stages (Figure 2). In the $\mathrm{E} 1$ state, SERCA can bind two $\mathrm{Ca}^{2+}$ ions to its high-affinity binding site in the M-domain, which is accessible from the cytosol (2 $\left.\mathrm{Ca}^{2+}-\mathrm{E} 1-\mathrm{ATP}\right)$. Through ATP binding to the $\mathrm{N}$-domain, the two $\mathrm{Ca}^{2+}$ ions are retained and occluded within the protein. As a result, the Asp351 within the P-domain can be phosphorylated by the transfer of the $\mathrm{Y}$-phosphate from ATP $\left(2 \mathrm{Ca}^{2+}-\mathrm{E} 1 \sim \mathrm{P}\right)$. At this state, the phosphoryl group can still be transferred back to the ADP. From this intermediate stage, it gets into a low $\mathrm{Ca}^{2+}$ affinity and ADP insensitive stage 2 $\mathrm{Ca}^{2+}-\mathrm{E} 2-\mathrm{P}$. In the rate-limiting transition from E1-P to E2-P, the P-domain reorientates together with the A-domain rotation towards the phosphorylation site and protects the phosphoryl-group from hydrolysis, and ADP dissociation. This rate limiting transition is accompanied by the disruption of the high affinity $\mathrm{Ca}^{2+}$ binding site and the release of $\mathrm{Ca}^{2+}$ into the SR lumen. The phosphorylated Asp351 is now accessible for the hydrolysis of the E2-P. Inorganic phosphate dissociates, and SERCA is regenerated to $\mathrm{E} 1$ for the next $\mathrm{Ca}^{2+}$ transport cycle $\mathrm{e}^{17,5,18}$. 


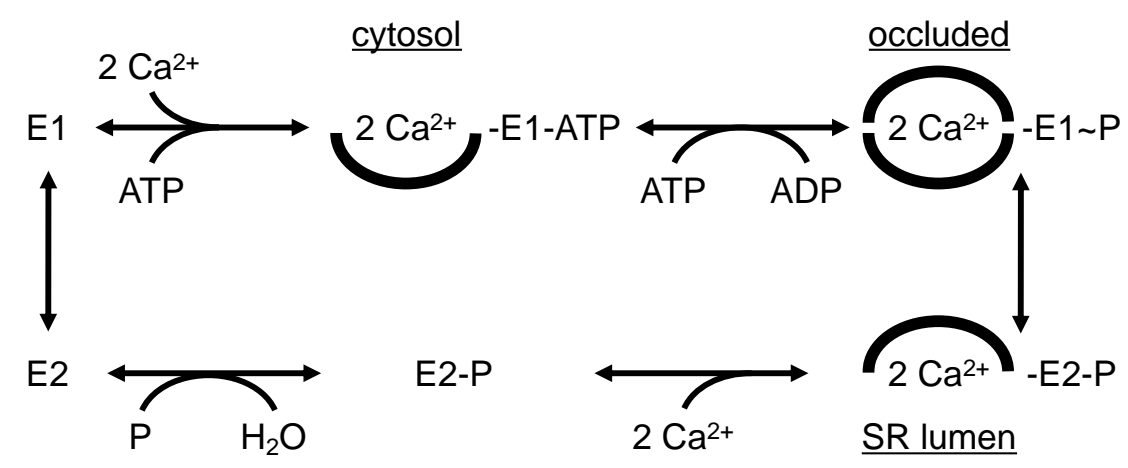

Figure 2 Scheme of the E1/E2 model for SERCA

Detailed explanation in the text. Scheme adapted from Wuytack et al. ${ }^{15}$

In the heart, SERCA2a controls the rate of $\mathrm{Ca}^{2+}$ transport into the SR and the $\mathrm{SR} \mathrm{Ca}^{2+}$ load, which determines the relaxation and contraction of a myocyte, respectively ${ }^{19}$.

Studies in genetically defined models elucidated the role of SERCA2a in $\mathrm{Ca}^{2+}$ homeostasis and cardiac contractility. Transgene mice overexpressing SERCA2a showed increased $\mathrm{Ca}^{2+}$ transport and enhanced contractility and relaxation ${ }^{20,21,22}$. No cardiac pathology was observed, suggesting toleration of the overexpressed SERCA2a. In contrast, with SERCA2a-deficiency, mice were not viable ${ }^{23}$. A heterozygous null SERCA2a mouse showed a $35 \%$ reduced SERCA2a protein expression but no cardiac pathology ${ }^{24}$. But under challenging conditions, heterozygous mice showed in a heart failure model with induced ventricular hypertrophy impaired intracellular $\mathrm{Ca}^{2+}$ homeostasis and decreased rates of cardiac contractility ${ }^{25}$. This suggests the necessity of two functional SERCA2a gene copies for normal heart function.

\subsection{Phospholamban}

Phospholamban (PLN) is a small 52 amino acids long tail-anchored membrane protein, which is mainly expressed in the heart ${ }^{26}$. It was discovered as 22000 Dalton protein when SR vesicles isolated from heart muscle showed increased calcium uptake after the addition of cyclic adenosine monophosphate (CAMP) and protein kinase A (PKA) ${ }^{27}$. This was related to the stimulatory effect mediated by the phosphorylation of PLN, which is colocalized with SERCA2a in SR membranes ${ }^{28}$. The analysis of $\mathrm{Ca}^{2+}$ kinetics demonstrated that dephosphorylated PLN diminishes the apparent affinity of SERCA2a for $\mathrm{Ca}^{2+} .{ }^{29}$

PLN is present in two different physiological states and forms monomers and pentamers. The active dephosphorylated form is a substantial fraction of the monomer that binds to and inhibits SERCA2a. The pentameric PLNs are mainly phosphorylated and are thought to be inactive or less active than monomeric PLN ${ }^{30}$. Pentameric PLN can bind to SERCA2a as well and is 
The cytosolic domain $I$ is further divided into domain $I_{a}$ (aa 1-20) and $I_{b}$ (aa 21-30). Domain $I_{a}$ consists of an alpha-helix and contains the phosphorylation sites, domain $\mathrm{I}_{\mathrm{b}}$ is suggested to be relatively unstructured ${ }^{33}$. Domain II (aa 31-52) forms an alpha-helix in the SR membrane (Figure 4). This domain II contains leucine and isoleucine residues, building an essential leucine-zipper motif for the pentamer assembly ${ }^{33}$. PLN pentamers are SDS resistant, and only the increase of detergent concentrations and boiling dissociates them into monomers. In immunoblotting, the PLN monomer shows a molecular weight of $6.1 \mathrm{kDa}$ and the pentamer of $22 \mathrm{kDa}^{34}$.

\subsection{PLN phosphorylation}

The region of PLN interacting with SERCA2a may involve amino acids 2 to 18 . This association is disrupted by phosphorylation of Ser10, Ser16, or Thr17 in PLN by the cAMP dependent protein kinase $C(P K C)$, the cAMP dependent protein kinase $A(P K A)$ or $\mathrm{Ca}^{2+}$-calmodulindependent protein kinase (CaMKII), respectively. This is due to the positive charge of the PLN cytosolic domain, and is partially neutralized by the phosphate group in these sites. Phosphorylation of PLN by the cAMP dependent protein kinase at Ser16 is associated with a local twist of the alpha-helix at positions 12 to 16 , resulting in conformation changes in the recognition unit of the protein. Also, domain II affect the $\mathrm{Ca}^{2+}$ ATPase activity by lowering its affinity for $\mathrm{Ca}^{2+} .33$

Prevention of Ser16 phosphorylation by mutagenesis (Ser16Ala) resulted in a reduced response to B-adrenergic stimulation in a mouse model ${ }^{35}$. At the same, no phosphorylated Thr17 could be detected. Animals with the Thr17 substitution for alanine, Ser16 phosphorylation was reactive for B-adrenergic stimulation. Maximum inhibition of SERCA2a was observed in mice overexpressing this PLN forms ${ }^{35}$.

The cAMP-dependent phosphorylation of PLN activates the SR $\mathrm{Ca}^{2+}$ by SERCA2a dissociation and contributes to the relaxation phase. The resulting increased $\mathrm{Ca}^{2+}$ levels accumulated by the SR would, in turn, lead to higher $\mathrm{Ca}^{2+}$ levels for the release from the SR during the contraction by binding to the contractile proteins. The critical role of PLN in the facilitation of Badrenergic stimulation responses was shown in PLN-deficient mice ${ }^{36}$. The treatment of these cardiomyocytes showed an attenuated response to B-adrenergic stimulation, indicating that PLN is a key target of this pathway. Also, other cardiac phosphoproteins like L-type $\mathrm{Ca}^{2+}$ channel and phospholemman (PLM) in the sarcolemma, troponin I and troponin C-proteins in the myofilaments and the $\mathrm{Ca}^{2+}$ release channel RyR2 have been shown to contribute to the stimulatory effects of B-adrenergic stimulation in the heart.

Compared to PLN-deficient mice, transgenic mice that overexpress PLN by 2-fold and 4-fold in the heart showed decreased rates of contraction and relaxation. These increased inhibitory 
effects were abolished by ß-adrenergic stimulation with the ß-agonist, which resulted in PLN phosphorylation and the relived inhibitory effects on SERCA2 $\mathrm{a}^{37}$. Interestingly, mice with 4fold higher PLN protein expression showed elevated levels of adrenaline and noradrenaline, suggesting a compensatory adaption, which would enhance the phosphorylation of PLN and the activation of SERCA2a.

\subsection{PLN dephosphorylation by PP1}

The main phosphatase dephosphorylating PLN is the protein phosphatase 1 (PP1). This enzyme accounts for $70 \%$ of PLN phosphatase activity towards the PKA phosphorylation site Ser16 and CamK2 phosphorylation site Thr1738. The PP1 activity is significantly increased during human heart failure ${ }^{39}$. Its overexpression in mice to a level similar to failing human hearts resulted in depressed contractility and diminished response to B-adrenergic stimulation, which resulted in hypertrophy, heart failure, and early death ${ }^{40}$.

PP1 is a holoenzyme containing the catalytic domain, including its phosphatase activity and a regulatory subunit, which targets the PP1 to different subcellular locations ${ }^{41}$. Its endogenous inhibitor I-1 and I-2 additionally regulate PP1 activity. I-1 gets activated on phosphorylation at Thr35 PKA resulting in an attenuated PP1 activity. Constitutively active I-2 showed in a cardiac-specific mouse model depressed PP1 activity, increased $\mathrm{Ca}^{2+}$ transients, and enhanced contractile parameters ${ }^{42}$. Additionally, increased PLN phosphorylation at Ser16 but not at Thr17 was found, suggesting a site-specific preference of PP1 for PLN. Therefore, PP1 is through PLN a crucial negative regulator of cardiac function.

Furthermore, it has been suggested that the heat shock protein 20 (Hsp20/HSPB6) regulates cardiac contraction via the regulation of PP1 and PLN ${ }^{43}$. Interestingly, cardiac overexpression of Hsp20 showed enhanced contractility ${ }^{43}$, whereas the knockdown of Hsp20 by microRNA320 showed depressed contractility ${ }^{44}$. These data suggest that Hsp20 directly binds to PP1 and inhibit its activity. As a direct consequence, PLN showed enhanced phosphorylation along with reduced PP1 protein expression in Hsp20 transgenic mice. Also, the increase of Hsp20 levels preserved contractile parameters and was suggested to be cardio protective ${ }^{43}$. Additionally, the HS-1 associated protein X-1 (HAX-1) is described as a direct interaction partner of $\mathrm{PLN}^{45}$. The proposed minimal binding region is from amino acids 16 to 22 , which includes the phosphorylation sides of PLN Ser16 and Thr17. It has been suggested that the binding between PLN and HAX-1 may control PLN activity ${ }^{45}$. Similar to the PLN/SERCA2a binding, the interaction with HAX-1 is diminished upon PLN phosphorylation.

Together these results support the beneficial effects of PLN phosphorylation and its regulation at multiple levels by modulating cardiac contractility and survival. 


\subsection{PLN mutations}

Several human mutations in the PLN gene have been identified to date. Two of these mutations are associated with increases in PLN inhibition of SERCA2, lowering its affinity for $\mathrm{Ca}^{2+}$. This is partially explained by the decreased phosphorylation of PLN by a cAMP-dependent protein kinase, preventing the release from SERCA2a. This chronic inhibition of SERCA2a by i.e. PLN mutation R14del or R9C leads to dilated cardiomyopathy (DCM) and premature death in families carrying this mutations ${ }^{46,47}$. The same phenotype was also found in transgenic mouse models carrying this mutations ${ }^{46,47}$. It is suggested that the endogenous PLNR14del mutant fails to co-localize with SERCA2a, resulting in enhanced contractility in mice due to sustained SERCA2a activity ${ }^{47}$. PLNR14del protein was shown to miss localize to the sarcolemma, where it interacts with $\mathrm{Na}, \mathrm{K}-\mathrm{ATPase}$, and causes additional cardiac remodeling.

\subsection{PLN/SERCA in heart failure}

In human and experimental heart failure in mice, the protein level and the activity of SERCA2a were found to be decreased, contributing to failing cardiac conditions ${ }^{48,49}$. Furthermore, the protein level of PLN remains unchanged and this results in an increased ratio of PLN:SERCA2a with more inhibited SERCA2a fractions. Additionally, phosphorylated PLN levels are decreased, resulting in a reduced SERCA2a activity ${ }^{49}$. This reduced PLN phosphorylation might be an effect of receptor downregulation or desensitization of the Badrenergic pathway ${ }^{50}$. Additional activation of phosphatases may also lead to higher PLN dephosphorylation and failing cardiac conditions ${ }^{40,51}$. The decrease in HAX-1, I-I or PKA phosphorylation contributes to reduced SERCA2a activity, whereas Hsp2O is significantly increased in failing hearts suggesting compensatory mechanisms.

In summary, the function of the PLN/SERCA2a modulators underlie regulatory mechanisms under physiological and pathophysiological conditions. There is a huge effort to reverse the contractile failure by i.e. SERCA2a gene therapy ${ }^{52}$ to restore the PLN/SERCA ratio and reestablish normal heart function. 


\subsection{Aim of the thesis}

Phospholamban is a central regulator for $\mathrm{Ca}^{2+}$ uptake into the $\mathrm{SR}$ and for cardiomyocyte contractility. During heart failure, $\mathrm{Ca}^{2+}$ handling is altered by reduced expression of SERCA2a while the PLN level is unchanged. Hence, the rate of relaxation and contractility is reduced. We hypothesize that PLN binds potentially to other important SER proteins and thereby regulates the reuptake of $\mathrm{Ca}^{2+}$ into the $\mathrm{SR}$.

This study was designed to explore novel interacting proteins of phospholamban by two different unbiased proteomic approaches. Here we used the bottom-up complexome profiling approach to identify protein-protein interactions in the native environment. The advances of the native membrane analysis by blue native gel electrophoresis were coupled with mass spectrometry analysis to identify migration profiles for multiple detected proteins. The overlap of migration profiles and the alignment by hierarchical clustering will allow the identification of novel cardiac protein complexes and assemblies, as well as the identification of previously unknown interactors.

In parallel, an alternative PLN targeted APEX2 proximity labeling assay was used to identify potential interactions in living neonatal rat cardiomyocytes. This approach will allow the labeling of proteins in the proximity of PLN by the covalent transfer of biotin-phenol and the extraction of labeled proteins after cell lysis. Besides the known interactors of phospholamban, we expect the enrichment of previously unknown.

Taken together, the identification of novel phospholamban interactors will elucidate new insights into the interactome and the regulatome of cardiac contractility. Furthermore, these interactions will be confirmed by complementary approaches to elucidate their functional role. 


\section{Material and Methods}

\section{$2.1 \quad$ Material}

\subsubsection{Buffer conditions}

CBFHH buffer

$\begin{array}{ll}\mathrm{NaCl} & 37 \mathrm{mM} \\ \mathrm{KCL} & 5.4 \mathrm{mM} \\ \mathrm{KH}_{2} \mathrm{PO}_{4} & 0.44 \mathrm{mM} \\ \mathrm{Na}_{2} \mathrm{HPO}_{4} \times 2 \mathrm{H}_{2} \mathrm{O} & 33.5 \mathrm{mM} \\ \text { Glucose }_{\text {HEPES }} & 5.6 \mathrm{mM} \\ \mathrm{MgSO}_{4} & 20 \mathrm{mM} \\ & 0.8 \mathrm{mM}\end{array}$

In 500 mL ddH $\mathrm{d}_{2} \mathrm{O}$, pH 7.4

NRCM cultivation medium

$\begin{array}{lc}\text { FBS } & 10 \%(\mathrm{v} / \mathrm{v}) \\ \text { 5-Bromo-2'-deoxyuridine } & 10 \mathrm{mM} \\ \text { Pen/Strep } & 1 \%(\mathrm{v} /) \\ \text { In } \mathbf{5 0 0} \text { mL DMEM-1 g/L D-glucose (Thermo Fisher) }\end{array}$

HEK293A cell culture medium

$\begin{array}{lc}\text { FBS } & 10 \%(\mathrm{v} / \mathrm{v}) \\ \text { L-glutamine } & 2 \mathrm{mM} \\ \text { Pen/Strep } & 1 \%(\mathrm{v} /) \\ \text { In } \mathbf{5 0 0 ~} \mathrm{mL} \text { DMEM-low glucose (Sigma-Aldrich) }\end{array}$

APEX2 biotinylation Quenching buffer

$\begin{array}{ll}\text { Trolox } & 5 \mathrm{mM} \\ \text { Sodium azide } & 10 \mathrm{mM} \\ \text { Sodium ascorbate } & 10 \mathrm{mM}\end{array}$

In 50 mL 1x PBS (Gibco), pH 7.4 
APEX2 biotinylation RIPA Quenching buffer

$\begin{array}{ll}\text { Trolox } & 5 \mathrm{mM} \\ \text { Sodium azide } & 10 \mathrm{mM} \\ \text { Sodium ascorbate } & 10 \mathrm{mM} \\ \text { Tris } \mathrm{HCl}, \mathrm{pH} \mathrm{7.4} & 50 \mathrm{mM} \\ \mathrm{NaCl} & 150 \mathrm{mM} \\ \text { Triton-X-100 } & 1 \%(\mathrm{v} / \mathrm{v}) \\ \text { Sodium doxycholate } & 0.5 \%(\mathrm{w} / \mathrm{v}) \\ \text { Sodium dodecylsulfate } & 0.2 \%(\mathrm{v} / \mathrm{v}) \\ \text { In } \mathbf{1 0 ~} \mathbf{m L} \text { dd }_{2} \mathrm{O} \text { pH 7.4, tablet Complete mini EDTA protease inhibitor (Roche) }\end{array}$

Biotin pulldown Urea buffer

Tris $\mathrm{HCl}, \mathrm{pH} 8$

Urea $2 \mathrm{mM}$

In $100 \mathrm{~mL} \mathrm{ddH} \mathrm{H}_{2} \mathrm{O}, \mathrm{pH} 8$

Biotin elution buffer

Biotin $2 \mathrm{mM}$

Sodium dodecylsulfate $\quad 2 \%(\mathrm{v} / \mathrm{v})$

In $1 \mathrm{~mL} \mathrm{ddH} \mathrm{H}_{2} \mathrm{O}, \mathrm{pH} 8$

iPSC culture medium

Thiazovivin $2 \mu \mathrm{M}$

In 500 mL StemMACS iPS-Brew stem cell culture media, Miltenyi Biotec

Cardio differentiation medium

Human recombinant albumin $\quad 0.5 \mathrm{mg} / \mathrm{mL}$

L-ascorbic acid 2-phosphate $\quad 0.2 \mathrm{mg} / \mathrm{mL}$

In 500 mL RPMI 1640 cell culture medium with Glutamax and HEPES (Thermo Fisher)

Cardio culture medium

B27

L-ascorbic acid 2-phosphate

In 500 mL RPMI 1640 cell culture medium with Glutamax and HEPES (Thermo Fisher) 
RIPA buffer

\begin{tabular}{|c|c|}
\hline Tris HCl, pH 7.4 & $50 \mathrm{mM}$ \\
\hline $\mathrm{NaCl}$ & $150 \mathrm{mM}$ \\
\hline NP40 & $1 \%(v / v)$ \\
\hline Sodium doxycholate & $0.5 \%(w / v)$ \\
\hline Sodium dodecylsulfate & $0.2 \%(\mathrm{v} / \mathrm{v})$ \\
\hline
\end{tabular}

5x SDS sample buffer

$\begin{array}{ll}\text { Tris } \mathrm{HCl}, \mathrm{pH} \mathbf{7 . 4} & 191.6 \mathrm{mM} \\ \text { Glycine } & 1.92 \mathrm{M} \\ \text { Sodium dodecylsulfate } & 34.69 \mathrm{mM} \\ \text { B-mercapto ethanol } & 5 \%(\mathrm{v} / \mathrm{v}) \\ \text { In } \mathbf{5 0 ~} \mathrm{mL} \text { dd } \mathrm{H}_{2} \mathrm{O} \text { pH } 8.3 & \end{array}$

CHAPS co-immunoprecipitation buffer

$\begin{array}{ll}\text { Tris HCl, pH 7.4 } & 50 \mathrm{mM} \\ \text { NaCl } & 150 \mathrm{mM} \\ \text { CHAPS } & 1 \%(\mathrm{w} / \mathrm{v}) \\ \text { EDTA } & 1 \mathrm{mM} \\ \text { 1x tablet Complete mini (Roche) } & \ln 10 \mathrm{~mL} \\ \text { 1x tablet PhosSTOP (Roche) } & \ln 10 \mathrm{~mL}\end{array}$

In $50 \mathrm{~mL} \mathrm{ddH} \mathrm{H}_{2} \mathrm{O}$, pH 7.4

Blue native homogenization buffer

\begin{tabular}{ll} 
Sucrose & $250 \mathrm{mM}$ \\
Tris HCl, pH 7.4 & $10 \mathrm{mM}$ \\
EDTA & $1 \mathrm{mM}$ \\
\hline 1x tablet Complete mini (Roche) & In $10 \mathrm{~mL}$ \\
1x tablet PhosSTOP (Roche) & In $10 \mathrm{~mL}$ \\
In $\mathbf{5 0 ~ m L ~ p H ~ 7 . 4 ~}$ &
\end{tabular}

Solubilization buffer A

$\begin{array}{ll}\mathrm{NaCl} & 50 \mathrm{mM} \\ \text { Imidazole } & 50 \mathrm{mM} \\ \text { EDTA } & 1 \mathrm{mM} \\ \text { Aminocaproic acid } & 1 \mathrm{mM} \\ \text { In } \mathbf{5 0 ~ m L ~ p H ~ 7 . 4} & \end{array}$


Blue native anode buffer

Imidazole

$25 \mathrm{mM}$

In 1 L pH 7.0

Blue native cathode buffer $B$

$\begin{array}{ll}\text { Tricine } & 50 \mathrm{mM} \\ \text { Imidazole } & 7.5 \mathrm{mM} \\ \text { Coomassie Blue G-250 } & 0.02 \%(\mathrm{w} / \mathrm{v})\end{array}$

In $1 \mathrm{~L} \mathrm{pH} 7.0$ (do not adjust)

Blue native cathode buffer $B / 10$

Tricine $50 \mathrm{mM}$

Imidazole

$7.5 \mathrm{mM}$

Coomassie Blue G-250

$0.002 \%(w / v)$

In $1 \mathrm{~L} \mathrm{pH} 7.0$ (do not adjust)

Coomassie Blue solution

Coomassie Blue G-250

Aminocaproic acid

$5 \%(\mathrm{w} / \mathrm{v})$

In $1 \mathrm{~mL}$

$500 \mathrm{mM}$

Mouse heart perfusion buffer

$\begin{array}{ll}\mathrm{NaCl} & 120.4 \mathrm{mM} \\ \mathrm{KCl} & 14.7 \mathrm{mM} \\ \mathrm{KH} 2 \mathrm{PO} 4 & 0.6 \mathrm{mM} \\ \mathrm{Na2HPO} 4 \text { x 2 H2O } & 1.2 \mathrm{mM} \\ \text { MgSO4 x 7 H20 } & 1.2 \mathrm{mM} \\ \text { HEPES } & 10 \mathrm{mM} \\ \text { NaHCO3 } & 4.6 \mathrm{mM} \\ \text { Taurin } & 30 \mathrm{mM} \\ \text { 2,3-Butanedione monoxime } & 10 \mathrm{mM} \\ \text { Flucose } & 5.5 \mathrm{mM}\end{array}$

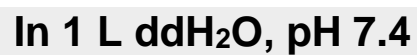

Mouse heart digestion buffer

Collagenase type II

$2 \mathrm{mg} / \mathrm{mL}$

$\mathrm{CaCl}_{2}$

$40 \sim \mathrm{M}$

In $50 \mathrm{~mL}$ perfusion buffer, $\mathrm{pH} 7.4$ 
Mouse heart STOP buffer

Bovine calf serum

$10 \%(\mathrm{v} / \mathrm{v})$

$\mathrm{CaCl} 2$

$12.5 \mu \mathrm{M}$

In $50 \mathrm{~mL}$ perfusion buffer, pH 7.4

Blocking buffer (immunofluorescence)

Bovine calf serum

$10 \%(\mathrm{v} / \mathrm{v})$

Triton-X-100

$0.2 \%(v / v)$

In 50 mL PBS (Gibco) 


\subsubsection{Antibodies}

\begin{tabular}{|c|c|c|c|c|c|c|c|c|}
\hline \multicolumn{9}{|c|}{ Primary antibodies } \\
\hline Protein & Species & Clonality & Clone & Company & Catalog no. & $\begin{array}{l}\text { Dilution } \\
\text { WB }\end{array}$ & $\begin{array}{c}\text { Dilution } \\
\text { IF } \\
\text { (NRCM/ } \\
\text { iPSC) }\end{array}$ & $\begin{array}{c}\text { Dilution } \\
\text { IF } \\
\text { (Histo) }\end{array}$ \\
\hline a-actinin & Mouse & Monoclonal & & Santacruz & A7811 & $1: 1000$ & & \\
\hline CAV3 & Mouse & Monoclonal & & $\begin{array}{l}\text { BD } \\
\text { Bioscience }\end{array}$ & 610421 & & & $1: 250$ \\
\hline CAV3 & Rabbit & Polyclonal & & Abcam & Ab2912 & $1: 2000$ & & \\
\hline GAPDH & Mouse & Monoclonal & & Biotrend & 5G4Mab6C5 & $1: 160000$ & & \\
\hline GFP & Mouse & Monoclonal & & Proteintech & HRP-66002 & $1: 5000$ & & \\
\hline Myc & Rabbit & Polyclonal & & $\begin{array}{l}\text { Sigma- } \\
\text { Aldrich }\end{array}$ & C3956 & & $1: 500$ & \\
\hline PLN & Mouse & Monoclonal & 2D12 & Abcam & Ab2865 & $1: 2000$ & $1: 500$ & $1: 250$ \\
\hline SERCA2a & Rabbit & Polyclonal & & Badrilla & $A 010-20$ & $1: 2000$ & $1: 500$ & $1: 250$ \\
\hline SLMAP & Mouse & Monoclonal & $2 \mathrm{~A} 7$ & Santa cruz & Sc-100957 & & $1: 250$ & \\
\hline SLMAP & Rabbit & Polyclonal & & $\begin{array}{l}\text { Sigma- } \\
\text { Aldrich }\end{array}$ & HPA002357 & $1: 1000$ & $1: 50$ & $1: 50$ \\
\hline V5 & Mouse & Monoclonal & $\begin{array}{l}\text { H00007871- } \\
\text { M08 }\end{array}$ & Invitrogen & R960-25 & $1: 1000$ & $1: 500$ & \\
\hline VDAC1 & Rabbit & Polyclonal & & Abcam & Ab15895 & $1: 2000$ & & \\
\hline
\end{tabular}




\begin{tabular}{|c|c|c|c|c|c|c|c|}
\hline \multicolumn{8}{|c|}{ Secondary antibodies } \\
\hline Protein & Species & Clonality & Company & Catalog no. & $\begin{array}{l}\text { Dilution } \\
\text { WB }\end{array}$ & $\begin{array}{c}\text { Dilution } \\
\text { IF } \\
\text { (VCM) }\end{array}$ & $\begin{array}{c}\text { Dilution } \\
\text { IF } \\
\text { (Histo) }\end{array}$ \\
\hline $\begin{array}{l}\text { IRDye } 800 \mathrm{CW} \\
\text { Donkey anti- } \\
\text { Mouse IgG }\end{array}$ & Donkey & Polyclonal & LI-COR & $\begin{array}{l}\text { P/N926- } \\
32212\end{array}$ & $1: 10000$ & & \\
\hline $\begin{array}{l}\text { IRDye 680RD } \\
\text { Donkey anti- } \\
\text { Mouse IgG }\end{array}$ & Donkey & Polyclonal & LI-COR & $\begin{array}{l}\text { P/N926- } \\
68072\end{array}$ & $1: 10000$ & & \\
\hline $\begin{array}{l}\text { IRDye } 800 \mathrm{CW} \\
\text { Donkey anti- } \\
\text { Rabbit }\end{array}$ & Donkey & Polyclonal & LI-COR & $\begin{array}{l}\text { P/N926- } \\
32213\end{array}$ & $1: 10000$ & & \\
\hline $\begin{array}{l}\text { IRDye 680RD } \\
\text { Donkey anti- } \\
\text { Rabbit IgG }\end{array}$ & Donkey & Polyclonal & LI-COR & $\begin{array}{l}\text { P/N926- } \\
68073\end{array}$ & $1: 10000$ & & \\
\hline $\begin{array}{l}\text { Goat anti- } \\
\text { mouse Alexa } \\
\text { Fluor } 633\end{array}$ & Goat & Polyclonal & $\begin{array}{l}\text { Thermo } \\
\text { Fisher }\end{array}$ & A-21052 & & $1: 1000$ & \\
\hline $\begin{array}{l}\text { Goat anti-rabbit } \\
\text { Alexa Fluor } 514\end{array}$ & Goat & Polyclonal & $\begin{array}{l}\text { Thermo } \\
\text { Fisher }\end{array}$ & A-31558 & & $1: 1000$ & \\
\hline $\begin{array}{l}\text { Goat anti-rabbit } \\
\text { STAR 635P }\end{array}$ & Goat & Polyclonal & Abberior & $2-0012-007-2$ & & $1: 1000$ & $1: 300$ \\
\hline $\begin{array}{l}\text { Goat anti- } \\
\text { mouse STAR } \\
635 \mathrm{P}\end{array}$ & Goat & Polyclonal & Abberior & $2-0002-007-5$ & & $1: 1000$ & $1: 300$ \\
\hline $\begin{array}{l}\text { Goat anti-rabbit } \\
\text { STAR } 580\end{array}$ & Goat & Polyclonal & Abberior & $2-0012-005-8$ & & $1: 1000$ & $1: 300$ \\
\hline $\begin{array}{l}\text { Goat } \quad \text { anti- } \\
\text { mouse } \\
580\end{array}$ & Goat & Polyclonal & Abberior & $2-0002-005-1$ & & $1: 1000$ & $1: 300$ \\
\hline
\end{tabular}




\subsubsection{Vectors}

\begin{tabular}{|l|l}
\hline DNA vector & Source \\
\hline pcDNA3-V5-APEX & Gift from Prof. Peter Rehling \\
\hline pdDNA3-YFP-PLN & Gift from Prof. Viacheslav Nikolaev \\
\hline pcDNA3-V5-APEX2-PLN & Cloning \\
\hline pcDNA3-V5-APEX2-PLN $\Delta(1-29)$ & Cloning \\
\hline pEF.myc.ER-E2-Crimson & $\begin{array}{l}\text { Gift from Benjamin Glick (Addgene plasmid } \\
\# 38770)\end{array}$ \\
\hline
\end{tabular}

\subsubsection{Cell lines}

Cell lines

Human embryonic kidney 293A cells $\quad$ CRL 1573, ATCC

Neonatal rat cardiomyocytes

WT1.14 /UMGi014-C.14)

Primary cell isolation

Stem Cell unit, University Medical Center, Göttingen

\subsubsection{Chemicals}

\begin{tabular}{ll}
\hline Name & Company \\
\hline 4-(2-hydroxyethyl)-1-piperazineethanesulfonic acid & Sigma \\
\hline Acetic Acid & Roth \\
\hline Acrylamide 2x & SERVA \\
\hline Acrylamide/Bis-Acrylamide solution & Roth \\
\hline Agarose & Lonza \\
\hline aminocaproic acid & Sigma \\
\hline Ammonium persulfate & Sigma \\
\hline Ampicillin & Roth \\
ampicillin & Roth \\
Biotin (d-) & SUPELCO \\
Biotinphenol & Iris Biotech \\
Bis-Acrylamide 2x & SERVA \\
Bovine Serum Albumin & Gibco \\
Bromdesoxyuridin & Sigma \\
Bromophenol blue & Sigma \\
\hline calcium chloride & Sigma \\
CHAPS & Roth \\
Collagenase Type 2 & Worthington \\
Coomassie Blue G-250 & SERVA \\
Di-sodiumhydrogen phosphate & Sigma \\
Digitonin & SERVA \\
Dimethylsulfoxide & Sigma \\
Dithiotreitol & Sigma \\
\hline Dulbeccos modified eagle medium & Biochrome \\
\hline & \\
\hline
\end{tabular}


EDTA 0.5 M solution

\section{EGTA}

Ethanol

EZ Blue Gel staining

Fetal Bovine Serium

Fetal Calf Serum

Glucose

Glycerol / glycerin

Glycin

HEPES

Hydrochloric acid fuming $\mathbf{3 7} \%$

Hydrogen peroxide

Imidazole

Isopropyl $\beta$-D-1-thiogalactopyranoside

kanamycin

L-Glutathione reduced

LB agar agar

LB Agar powder

\section{LB Medium}

Lysozym from chicken egg

Magensium sulfate monohydrate

Methanol

Milk, powdered blotting grade

Nonidet P40

ortho-Phosphoric acid $89 \%$

Penicillin-Streptomycin

Phenylmethylsulfonyl fluoride

Phosphate bufferd saline

PhosSTOP tablet

Pierce Bovine Serum Albumin Standard

Potassium chloride

Potassium dihydrogenphosphate

Protease Inhibotor tablet

rat tail collagen type 1

Roti ${ }^{\circledR}$ Block

SILAC DMEM Flex Media

Sodium ascorbate

Sodium azide

Sodium chloride

Sodium chloride

Sodium deoxycholate

Sodium dodecylsulfate

Sodium dodecylsulfate ( $20 \%$ solution)

Sodium hydroxide solution

B-mercaptoethanol

Sucrose
AppliChem

Sigma

Merck Millipore

Sigma

Invitrogen

Thermo Fisher

Sigma

Roth

Roth

Roth

Roth

Sigma

Sigma

Sigma

Roth

Sigma

Applichem

AppliChem

AppliChem

Sigma

Sigma

Roth

Sigma

USB

Merck Millipore

Sigma

Sigma

Gibco, Life technologies

Roche

Thermo Fisher

Sigma

Sigma

Roche

Corning

Roth

Thermo Fisher

Sigma

Merck Millipore

Roth

Sigma

Sigma

Roth

Roth

Merck Millipore

Sigma

Roth 
SYBR Green

Tetramethylethylenediamine

Tricine

Tris(hydroxymethyl)aminomethane

Triton-X-100

Trolox

Trypsin

Tween-20

Urea
Quantan Biosciences

Roth

Sigma

Roth

Sigma

Sigma

Sigma

Sigma

Roth

\subsubsection{Kits}

Name

cDNA synthesis

Dynabeads protein $\mathbf{G}$

midi plasmid isolation

mini Plasmid isolation

Nucleo Spin Gel and PCR clean-up

Pierce $^{\mathrm{TM}}$ BCA Protein Assay Kit

Pierce $^{\mathrm{TM}}$ Biotinylated Protein

Interaction Pull-Down Kit

QIAShredder kit

RNA extraction kit

\section{Company}

Quanta Biosciences

Thermo Fisher

Qiagen

Qiagen

Macherey-Nagel

Thermo Fisher

Life technologies

Qiagen

Qiagen

\subsubsection{Instruments}

Name

Absorption/fluorescence plate reader

Blotting Station

Breeding cabinet (E.coli), Inforst HAT

Breeding cabinet (cell culture), HERAcell 150i

Centrifuge

Confocal microscope

DNA Camera Hero Doc plus

Potter homogenizer RW20 digital

Electrophoresis Power Supply

Magnet-stirrer RCT standard

Confocal Microscope LSM880

pH Electrode

Photometer

Pipetboy

Pipetts

Rotating wheel PDR-35

Shaker table Duomax 1030

STED microscope

\section{Company}

Teacan

AA Hoeffer

Ecotron

Thermo Fisher

Sorva

Zeiss

Herolab

IKA

Bio-Rad

IKA

Zeiss

Hannah Instruments

Eppendorf

Integra

Eppendorf/Gilson

Grant-bio

Heidolph

Leica 
Table top centrifuge HERAEUS Pico 17

Table top centrifuge HEAREUS Fresco

Thermocycler, labcycler

Thermomixer

Ultra turrax D1

Ultracentrifuge

Vortexer

Waterbath

WB-detection cabinet
Thermo Fisher

Thermo Fisher

SensoQuest

Eppendorf

Miccra

Beckman coulter

Scientific industries

Memmert

Li-Cor

\subsubsection{Consumables}

\begin{tabular}{|c|c|}
\hline Name & Company \\
\hline Eppendorf Tubes $1.5 \mathrm{ml}$ & Eppendorf \\
\hline Eppendorf Tubes $2.0 \mathrm{ml}$ & Eppendorf \\
\hline Reaction tube $15 \mathrm{ml}$ & Sarstedt \\
\hline Reaction tube $50 \mathrm{ml}$ & Sarstedt \\
\hline PCR tube $200 \mu l$ & Sarstedt \\
\hline Pipett tips $10 \mu \mathrm{l}$ & Sarstedt \\
\hline Pipett tips $200 \mu l$ & Sarstedt \\
\hline Pipett tips $1000 \mu l$ & Sarstedt \\
\hline Pipette 5 ml & Sarstedt \\
\hline Pipette 10 ml & Sarstedt \\
\hline Pipette 25 ml & Sarstedt \\
\hline Pipette 50 ml & Sarstedt \\
\hline Gel Loader Tips & Sarstedt \\
\hline Pipett filter tips $10 \mu l$ & Sarstedt \\
\hline Pipett filter tips $200 \mu \mathrm{l}$ & Sarstedt \\
\hline Pipett filter tips $1000 \mu l$ & Sarstedt \\
\hline Cellculutre flask T25 & Sarstedt \\
\hline Cell culture flask T75 & Sarstedt \\
\hline Cell culture flask T175 & Sarstedt \\
\hline 6-well plate & Greiner \\
\hline 12-well plate & Greiner \\
\hline 24-well plate & Greiner \\
\hline 96-well plate & Greiner \\
\hline Immobilon-P PVDF Membrane $0.45 \mu \mathrm{m}$ & Merck Millipore \\
\hline Immobilon-FL PVDF Membrane 0.45 m & Merck Millipore \\
\hline Sterile filter & Milipore \\
\hline Sterile filter for buffer & Milipore \\
\hline Cannula, 27G & Braun \\
\hline Cannula, 20G & Braun \\
\hline Cell scraper & Sarstedt \\
\hline Low profile 96 well plate & Bio-Rad \\
\hline syringe $1 \mathrm{ml}$ & Braun \\
\hline
\end{tabular}


ultracentrifuge tube I

ultracentrifuge tube II

\subsubsection{Software}

\begin{tabular}{ll} 
Name & Company \\
\hline Word & Microsoft Inc. \\
\hline Powerpoint & Microsoft Inc. \\
\hline Excel & Microsoft Inc. \\
\hline Outlook & Microsoft Inc. \\
\hline Fijilmage & https://imagej.net/Fiji \\
\hline Prism (Version 7.03) & Graphpad \\
\hline Cluster3.0 & Open source clustering software \\
\hline Mendeley & www.mendeley.com \\
\hline Bio-Rad CFX Manager & Bio-Rad \\
\hline
\end{tabular}




\subsection{Methods}

\subsubsection{Mouse husbandry}

The phospholamban germline knockout (PLN KO) mouse (C57BI/6N) was backcrossed for ten generations in the B6N background. Mice were housed at the Max Planck Institute (MPI) for experimental medicine Göttingen and the central animal facility (ZTE) of the University Medical Center Göttingen. The cages were part of a ventilated system (IVC racks), equipped with woodchips and nesting material. The rooms were kept at $20{ }^{\circ} \mathrm{C}$ to $24{ }^{\circ} \mathrm{C}$ and $45 \%$ to $65 \%$, with a light/dark cycle of $12 \mathrm{~h}$ dark and $12 \mathrm{~h}$ light. Mice had access to water and food ad libitum. Mice for breeding were genotyped using ear punch tissue from P21. For organizing the breeding at the MPI, the PYRAT software was used. All animal procedures were reviewed by the Institutional Animal Care and Use Committee by the University Medical Center Göttingen and approved by veterinarian state authority (LAVES, Oldenburg, Germany) in compliance with the humane care and use of laboratory animals (33.9-42502-04-16/2102). Unless indicated otherwise, male mice aged 12-16 weeks were used for experimental procedures.

\subsubsection{Mouse Genotyping}

For mouse genotyping, the ear punch tissue was lysed according to the manufacturer's protocol (DirectPCR Lysis Reagent Ear, Peqlab). $1 \mu$ l of crude lysate was used for polymerase chain reaction (PCR) in a Thermocycler (Sensoquest) according to protocols below (Table 2,Table 3). The obtained PCR products were supplemented with 10x loading dye and separated on a $2 \%$ agarose gel by gel electrophoresis for $40 \mathrm{~min}$ at $110 \mathrm{~V}$ in $1 \mathrm{x}$ TAE buffer. The expected PCR product sizes are given in Table 4.

Table 2 Genotyping protocol PCR mix

\begin{tabular}{|c|c|}
\hline & PLN KO \\
\hline Manoo Taq 5x buffer & $5 \mu l$ \\
\hline $\mathrm{MgCl} 2$ (50 mM) & $1.5 \mu l$ \\
\hline dNTP's (10 mM each) & $0.5 \mu \mathrm{l}$ \\
\hline Primer Fwd (PLN1F) (10 $\mu \mathrm{M})$ & 0.75 \\
\hline Primer Fwd II (NEO3) (10 $\mu \mathrm{M})$ & 0.75 \\
\hline Primer Rev (IMU8) (10 $\mu \mathrm{M})$ & $1 \mu l$ \\
\hline DNA & $1 \mu l$ \\
\hline $\mathrm{H}_{2} \mathrm{O}$ & $14.3 \mu \mathrm{l}$ \\
\hline 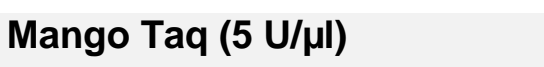 & $0.2 \mu \mathrm{l}$ \\
\hline Total reaction volume & $25 \mu l$ \\
\hline
\end{tabular}


Table 3 PCR protocol for PLN KO mouse genotyping

\begin{tabular}{lll} 
& Temperature & Time \\
\hline Preincubation & $95^{\circ} \mathrm{C}$ & $2 \mathrm{~min}$ \\
Denaturation & $95^{\circ} \mathrm{C}$ & $1 \mathrm{~min}$ \\
Annealing & $58^{\circ} \mathrm{C}$ & $30 \mathrm{sec}$ \\
Elongation & $72^{\circ} \mathrm{C}$ & $1 \mathrm{~min}$ \\
Number of cycles & $35 \mathrm{x}$ & \\
Final elongation & $72^{\circ} \mathrm{C}$ & $7 \mathrm{~min}$ \\
End & $4^{\circ} \mathrm{C}$ & hold
\end{tabular}

Table 4 PLN KO mouse genotyping primer

$\begin{array}{lll}\text { Gene } & \text { Primer sequence } & \text { Expected sizes } \\ \text { PIn wt fwd I } & \text { 5'-CCAGTCTGGGGAATAAATGG-3' } & \text { WT: } 900 \text { bp } \\ \text { PIn Neo fwd II } & \text { 5'-TCCTCGTGCTTTACGGTATC-3' } & \text { Het: } 400 \text { and } 900 \text { bp } \\ \text { PIn rev } & \text { 5'-TGTGGGTTTGCAAAGTTAGGC-3' } & \text { KO: } 400 \text { bp }\end{array}$

\subsubsection{Cloning of V5-APEX2-PLN and V5-APEX2-PLN $\Delta(1-29)$}

The V5 epitope tagged APEX2 pcDNA3 construct was a gift from Prof. Peter Rehling (Institute for Biochemistry, Göttingen). The wildtype canine phospholamban (PLN) cDNA sequence was a gift from Prof. Viacheslav Nikolaev (UKE, Hamburg). The PLN cDNA was amplified according to the manufacturer's protocol (In-Fusion HD Cloning Kit, Clonetech) using the following primers:

Fwd-5'-GGGGCAGCGGCTCGAGCGAGAAAGTTCAATACCTCAC-3'

Rev-5'-TAGATGCATGCTCGAGTCAGAGAAGCATGAC-3'

The pcDNA3-V5-APEX2 vector was linearized using Xhol (NEB) restriction enzyme digestion. The PLN PCR product and the linearized vector were cleaned up using the Nucleo Spin Gel and PCR clean-up (Macherey-Nagel) following the manufacturer's protocols. The PLN PCR fragment was cloned into the linearized V5-APEX2 vector by ligation (In-Fusion HD Cloning Kit, Clonetech). The vector was transformed into Stellar competent cells (Clonetech) and plated on LB-agar plates with $100 \mu \mathrm{g} / \mathrm{ml}$ ampicillin for overnight at $37^{\circ} \mathrm{C}$. Ampicillin resistant clones were picked and amplified in $5 \mathrm{~mL}$ LB medium containing ampicillin overnight at $37^{\circ} \mathrm{C}$. Plasmids were isolated using the QIAprep Miniprep kit (Qiagen) according to the 
manufacturer's protocol. Plasmids were sequenced by Microsynth Seqlab GmbH (Göttingen) using standard primers.

CMV-fwd: 5'-CGCAAATGGGCGGTAGGCGTG-3'

BGH-rev: 5'-TAGAAGGCACAGTCGAGG-3'

The DNA plasmid carrying the deletion of the cytosolic domain of PLN (pcDNA3-V5-APEX2PLN $\Delta(1-29)$ ) was amplified by InFusion-PCR according to manufacturer's protocol (In-Fusion HD Cloning Kit, Clonetech) using the following primer pair:

Fwd-5'-AACCTATTTATCAATTTCTGTCTCATC-3'

Rev-5'-ATTGATAAATAGGTTGCTCGAGCCGCTGCCCCC-3'

The resulting construct was ligated, amplified and sequenced as described.

\subsubsection{Adenoviral constructs for transduction}

Adenoviral vectors and particles were custom made by Sirion Biotech (Martinsried, Germany). For the generation of adenviral vectors, pcDNA3-V5-APEX2-PLN and pcDNA3-V5-APEX2$\mathrm{PLN} \Delta$ (1-29) were cloned into the adenoviral shuttle plasmid pO6A5-CMV. By recombination, these constructs were transferred into the adenoviral expression vector SIR-BAC-Ad5. After restriction enzyme digestion with Pacl, vector DNA was transfected into HEK293 cells for adenoviral particle production. The expression constructs were designed for constitutive expression under the CMV promoter with eGFP as expression control separated by an internal ribosomal entry side (IRES) sequence (Figure 5). Purified adenoviral particles serotype V Ad5APEX2-PLN-IRES-eGFP and Ad5-APEX2-PLNA(1-29)-IRES-eGFP were used for the transduction of neonatal rat cardiomyocytes (NRCMs).

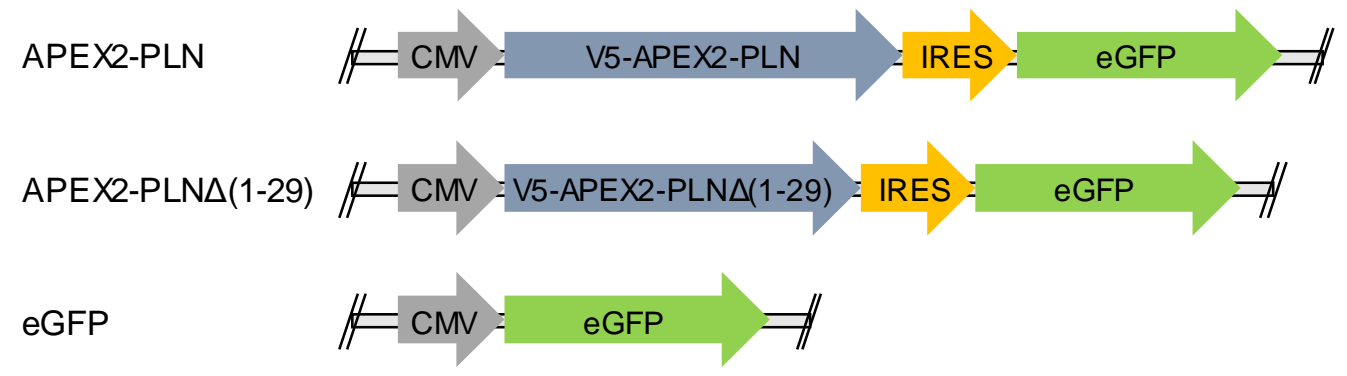

Figure 5 Adenoviral vector constructs for transduction of NRCMs

For APEX2 proximity labeling in NRCMs, adenoviral vectors carrying APEX2-PLN and APEX2-PLNA(129) were generated by Sirion Biotech (Martinsried, Germany). The expression constructs were designed for constitutive expression under the CMV promoter with eGFP as expression control separated by an internal ribosomal entry side (IRES) sequence. Adenoviral particles subclass V (Ad5) were used for transduction to target APEX2 constructs into NRCMs. 


\subsubsection{SDS gel electrophoresis}

Samples for detection in immunoblotting were lysed and homogenized in RIPA buffer containing protease and phosphatase inhibitors (Roche). Depending on the sample, mechanical disruption was performed by three times $10 \mathrm{sec}$ with the Miccra D-1 ultra turrax (heart tissue), 50 strokes using a potter homogenizer at $2000 \mathrm{rpm}$ (isolated cardiomyocytes) or 10 strokes with a 27-gauge needle (cultivated HEK or NRCMs). The homogenates were solubilized for $1 \mathrm{~h}$ at $4{ }^{\circ} \mathrm{C}$ with rotation, followed by centrifugation at $13000 \mathrm{xg}$ at $4{ }^{\circ} \mathrm{C}$. After collecting the supernatant, protein concentrations were determined using Pierce BCA protein Assay Kit (Thermo Fisher). For immunoblotting, $10 \mu \mathrm{g}$ of protein was supplemented with $5 \mathrm{x}$ SDS sample buffer and subjected on $4-20 \%$ gradient Tris-Glycine-SDS gel (Thermo Fisher) or $10 \%$ Tris-SDS acrylamide gels. The samples were resolved at constant $200 \mathrm{~V}$ for approximately $50 \mathrm{~min}$ or at $80 \mathrm{~V}$ for $30 \mathrm{~min}$ and $120 \mathrm{~V}$ for $90 \mathrm{~min}$, respectively. A Tris-Glycine buffer was used as anode and cathode buffer. For molecular weight estimation, a pre-stained protein marker was used (Thermo Fisher).

\subsubsection{Immunoblotting}

For immunoblotting, blue native or SDS gel was equilibrated in transfer buffer and proteins were transferred onto polyvinylidene fluoride (PVDF) membranes $(0.45 \mathrm{~mm}$, Immobilon-FL, Merck Millipore). In short, PDVF membranes were activated in methanol and equilibrated in transfer buffer. The protein transfer was performed using an electrophoretic transfer cell at constant $100 \mathrm{~V}$ for $1 \mathrm{~h}$ for SDS gels and $2 \mathrm{~h}$ for BN gels at $4{ }^{\circ} \mathrm{C}$. After blotting, PVDF membranes were blocked in 5\% non-at milk (w/v) in TBS-T for $1 \mathrm{~h}$ at room temperature. Primary antibodies were diluted in 5\% milk in TBS-T and membranes were incubated overnight at $4{ }^{\circ} \mathrm{C}$. After washing the PVDF membranes $3 x$ for $10 \mathrm{~min}$ with TBS-T, they were incubated with anti-mouse or anti-rabbit fluorescent secondary antibodies, diluted 1:10000 in 1x Rotiblock in TBS-T (Roth), for a minimum period of $1 \mathrm{~h}$ at room temperature with agitation. Fluorescence signals were captured with the Odyssey CLX imaging system (LI-COR). For analysis of biotinylated proteins, PVDF membranes were incubated with fluorescent-labeled streptavidin (RD680, LI-COR) for $1 \mathrm{~h}$ at RT in the dark. Signal intensities were analyzed by Image Studio Lite Version 5.2 (LI-COR) for quantification in Microsoft Excel.

\subsubsection{Co-Immunoprecipitation of phospholamban interacting proteins}

Ventricular tissue from PLN WT and KO mice was collected after perfusion with isotonic $\mathrm{NaCl}$ solution for 2 min using the Langendorff perfusion system. After that, samples were snap frozen in liq. $\mathrm{N}_{2}$, and stored at $-80^{\circ} \mathrm{C}$ until further analysis. For co-immunoprecipitation, 
ventricular tissue was thawed on ice and homogenized in non-denaturing Co-IP lysis buffer using the D-1 ultra turrax (Miccra). Homogenates were solubilized for $1 \mathrm{~h}$ at $4{ }^{\circ} \mathrm{C}$ with rotation, followed by a centrifugation step at $5000 \mathrm{xg}$ for $20 \mathrm{~min}$ at $4^{\circ} \mathrm{C}$. Supernatant of lysates were collected and protein concentration was determined using the Pierce BCA-protein assay (Thermo Fisher). For the antibody-lysate binding, lysates containing $500 \mu \mathrm{g}$ of protein were incubated with $4 \mu \mathrm{l}$ of anti-PLN primary antibody (ab2865, abcam) in a total volume of $1 \mathrm{~mL}$ Co-IP lysis buffer for overnight at $4{ }^{\circ} \mathrm{C}$ with rotation. For immunoprecipitation, $30 \mu \mathrm{l}$ of $50 \%$ slurry magnetic beads (Dynabeads Protein G, Thermo Fisher) were washed with $500 \mu \mathrm{l}$ Co-IP lysis buffer and incubated with the antibody-lysate mix for $1.5 \mathrm{~h}$ at $4{ }^{\circ} \mathrm{C}$ with rotation. After that, samples were placed on a magnet (DynaMag2 magnet, Thermo Fisher) and beads-antibodyantigen complex was washed three times with ice-cold $1 \mathrm{~mL}$ Co-IP lysis buffer for $10 \mathrm{~min}$ each at $4{ }^{\circ} \mathrm{C}$ with rotation. To avoid co-elution of proteins bound to the tube wall, the beads-antibodyantigen complex was resuspended in $200 \mu \mathrm{l}$ Co-IP lysis buffer and transferred into a new tube. For elution of the antibody-antigen complex, $50 \mu \mathrm{l}$ of 2x SDS sample buffer were added. After vigorous mixing for $10 \mathrm{~min}$ at $\mathrm{RT}$, the supernatant was collected, and samples were analyzed by SDS-PAGE and immunoblotting.

\subsubsection{Cell cultivation of HEK293A}

HEK293A cells (ATCC) were cultivated in T75 flasks (Greiner) in Dulbecco's Modified Eagle's Medium (DMEM low glucose, Sigma) supplemented with 10\% (v/v) fetal bovine serum (FBS, Thermo Fisher) $2 \mathrm{mM}$ L-glutamine GlutaMAX (Sigma-Aldrich) and penicillin/streptomycin (Sigma-Aldrich). Cells were cultivated under humidified conditions with $5 \% \mathrm{CO}_{2}$ at $37^{\circ} \mathrm{C}$ (Heracell VIOS 250i, Thermo Fisher) and passaged every 3-4 days using trypsin/EDTA (Sigma) digestion.

\subsubsection{Isolation of neonatal rat cardiomyocytes}

For the isolation and purification of neonatal rat cardiomyocytes (NRCMs) hearts from 0 to 3 days old wistar rats were collected and washed in cooled $\mathrm{CBFHH}$ buffer. After removing the atria, ventricles were washed in $\mathrm{CBFHH}$ buffer and cut in half. By using a combination of enzymatic and mechanical dissociation cardiomyocytes were dissociated from the ventricles (Neonatal Heart Dissociation Kit, Miltenyi Biotec) according to the manufacturer's protocol. In short, minced ventricular tissue was applied to a stainless-steel filter mesh (pore size $250 \mu \mathrm{m}$, Thermo Fisher) to remove larger particles. Cells in the resulting suspension were pelleted by centrifugation at $60 \times \mathrm{g}$ for $20 \mathrm{~min}$ at $4{ }^{\circ} \mathrm{C}$ (HERAEUS Multifuge X1R, Thermo Fisher) and resuspended in 1x PBS supplemented with Pen/Strep. For the specific enrichment of 
cardiomyocytes, a two-layer Percoll (GE Healthcare) density gradient with $63 \%$ and $40.5 \%$ was overlayered with the cell suspension and centrifuged at $3000 \mathrm{xg}$ for $30 \mathrm{~min}$ at room temperature without deceleration (HERAEUS Multifuge X1R, Thermo Fisher). Cardiomyocytes were separated from fibroblasts based on their different density and harvested from the Percoll interface between $63 \%$ and $40.5 \%$ using a $10 \mathrm{~mL}$ glass pipette. The enriched cardiomyocytes were suspended in prewarmed NRCM cultivation medium without FBS (Thermo Fisher) and immediately pelleted at $1200 \mathrm{xg}$ for $5 \mathrm{~min}$ at room temperature. Finally, cardiomyocytes were suspended in warm NRCM cultivation medium supplemented with heat inactivated $10 \%$ (vol/vol) FBS.

To determine the cell concentration, $50 \mu$ of cell suspension was mixed with $50 \mu$ trypan blue solution (Sigma-Aldrich) and cardiomyocytes were counted using a Neubauer improved chamber (LO-Labor Optik). NRCMs were seeded on collagen coated 6-well culture plates $\left(5 \times 10^{5}\right.$ cells/well) or glass cover slides (12-well culture plates, $2.5 \times 10^{5}$ cells/well) for the APEX proximity labeling or immune fluorescence staining, respectively, and cultivated under humidified conditions with $5 \% \mathrm{CO}_{2}$ at $37^{\circ} \mathrm{C}$. The $\mathrm{NRCM}$ cell culture medium was replaced every second day.

\subsubsection{NRCM cultivation in SILAC medium}

For ratiometric stable isotope labeling with amino acids in cell culture (SILAC), SILAC DMEM Flex Media lacking lysine and arginine (Thermo Fisher) was supplemented with $10 \%$ heat inactivated FBS, $100 \mu \mathrm{M}$ BRDU, $0.11 \mathrm{~g} / \mathrm{L}$ sodium pyruvate, $1 \mathrm{~g} / \mathrm{L}$ glucose, $100 \mathrm{U} / \mathrm{mL}$ penicillin/streptomycin mix and the respective radiolabeled amino acids $\mathrm{L}$-arginine and $\mathrm{L}$ lysine. Heavy SILAC media contained L-arginine $\left[{ }^{13} \mathrm{C}_{6},{ }^{15} \mathrm{~N}_{4}\right] \mathrm{HCl}($ Arg-10) and L-lysine $\left[{ }^{13} \mathrm{C}_{6},{ }^{15} \mathrm{~N}_{2}\right] \mathrm{HCl}$ (Lys-8) (Eurisotop); medium SILAC media contained L-arginine $\left[{ }^{13} \mathrm{C}_{6}\right] \mathrm{HCL}$ (Arg6) and L-lysine-4,4,5,5-d4 (Lys-4) (Eurisotop); light SILAC media contained light L-arginine (Arg-0) and L-lysine (Lys-0) using DMEM-media (Biochrome). After isolation and purification of NRCMs (as described above), the cells were suspended in the respective SILAC cultivation media without FBS and immediately pelleted at $1200 \times \mathrm{g}$ for $5 \mathrm{~min}$ at room temperature. The pelleted NRCMs were suspended in SILAC cultivation media supplemented with FBS, and $5 \times 10^{5}$ cells were seeded for APEX2 proximity labeling on collagen coated 6 -well culture plates. NRCMs were cultivated under humidified conditions with $5 \% \mathrm{CO}_{2}$ at $37^{\circ} \mathrm{C}$ for 13 days in $2 \mathrm{~mL}$ SILAC cell culture medium to achieve an incorporation of radiolabeled L-arginine and L-lysine of $>95 \%$. 


\subsubsection{Human induced pluripotent stem cell differentiation}

Differentiation and cultivation of human induced pluripotent stem cells (iPSC) was performed by Dr. Lukas Cyganek and the Stem Cell Unit (University Medical Center, Göttingen). Directed differentiation of human iPS cells into a ventricular phenotype was performed via WNT signaling modulation, as described previously ${ }^{53}$. The differentiation of iPS cells was initiated at 80-90\% confluence on Matrigel coated 6-well-plates with cardio differentiation medium and sequential treatment with $4 \mu \mathrm{M}$ CHIR99021 (Merck Millipore) for $48 \mathrm{~h}$, followed by $5 \mu \mathrm{M}$ IWP2 (Merck Millipore) for additional $48 \mathrm{~h}$. Medium was changed to cardio culture medium at day 8 . To prevent detachment and enhance selection, differentiated cultures were digested with collagenase B (Worthington Biochemical) and 0.25\% Trypsin/EDTA at day 12-15 (Thermo Fisher) and replated in lower cell density on Matrigel coated plates. Cardiomyocyte selection was performed using cardio selection medium for 5 days and a medium change every second day. For further maturation and cultivation, iPSC derived cardiomyocytes were cultured in cardio culture medium with medium change every 2-3 days. For protein analysis, cultured IPSC derived cardiomyocytes were washed with 1x PBS, harvested with a cell scraper (Sarstedt), pelleted at $13000 \mathrm{xg}$ for $10 \mathrm{~min}$ at $4{ }^{\circ} \mathrm{C}$, and frozen in liquid $\mathrm{N}_{2}$ and stored at $-80^{\circ} \mathrm{C}$ for further analysis. For immunofluorescence and immunoblot analysis the human IPSC lines isWT1.14 (UMGi014-C.14) were used.

\subsubsection{Transfection of HEK293A cells and NRCM}

For transfection of HEK293A cells and NRCM, cells were seeded in 6-well culture plates at a density of $2 \times 10^{5}$ cells per well $24 \mathrm{~h}$ prior to plasmid transfection. After reaching a confluency of approximately $70 \%$, the cells were plasmid transfected with Lipofectamine 3000 (Thermo Fisher). Therefore, $2 \mu \mathrm{g}$ of plasmid DNA and P3000 (2 $\mu \mathrm{l} / \mu \mathrm{g}$ DNA) were added to DMEM medium without supplements. In parallel, Lipofectamine $3000(1 \mu \mathrm{l} / \mu \mathrm{g}$ DNA) was added to DMEM medium without supplements and vortexed for 2-3 sec. Both DNA/P3000 and Lipofectamine 3000 containing DMEM were mixed in a ratio of 1:1 and incubated for $10 \mathrm{~min}$ at room temperature. Cells were washed once with 1x PBS, before the DNA-Lipofectamine complex was added. After $24 \mathrm{~h}$ of incubation, the cells were harvested for further experiments. In preparation for immunofluorescence staining, cells were seeded in 12-well culture plates on glass cover slides $\left(\varnothing 18 \mathrm{~mm}\right.$ ) with a density of $1 \times 10^{5}$ cells per well and, after $24 \mathrm{~h}$, transfected with $1 \mu \mathrm{g}$ plasmid DNA following the protocol described above. 


\subsubsection{Adenoviral transduction of NRCMs}

For NRCM transduction, adenoviral particles were diluted in $1 \mathrm{~mL}$ cultivation medium without FBS with the respective multiplicity of infection (MOI) and applied to the cells. After $2 \mathrm{~h}, 1 \mathrm{~mL}$ of cultivation medium with FBS was added and NRCMs were cultured for $48 \mathrm{~h}$ under humidified conditions with $5 \% \mathrm{CO}_{2}$ at $37^{\circ} \mathrm{C}$. The adenoviral transduction was monitored by eGFP expression using a fluorescence microscope (Axio Vert.A1, Zeiss).

For testing of the required multiplicity of infection, NRCMs were cultivated for 6 days before transduction. For ratiometric APEX2 proximity labeling, NRCMs were cultivated for 13 days in heavy, medium and light SILAC-medium before adenoviral transduction using a MOI of 10 and allowing for $48 \mathrm{~h}$ of expression.

\subsubsection{APEX2 Proximity Labeling}

APEX2 proximity labeling in HEK293A and NRCMs was conducted according to the published protocol by Hung et al. ${ }^{54}$. Therefore, HEK293A cells were transfected with $2 \mu \mathrm{g}$ and NRCMs with $4 \mu \mathrm{g}$ of plasmid for $24 \mathrm{~h}$ or NRCMs were transduced with adenoviral particles at a MOI of 10 for $48 \mathrm{~h}$ as described above. Prior to biotinylation, cells were incubated in $1 \mathrm{~mL}$ cultivation medium supplemented with $500 \mu \mathrm{M}$ biotin-phenol for $30 \mathrm{~min}$ at $37^{\circ} \mathrm{C}$. To initiate the biotinylation, a final concentration of $1 \mathrm{mM} \mathrm{H}_{2} \mathrm{O}_{2}$ was added with agitation for $1 \mathrm{~min}$ at room temperature. The reaction was stopped by washing the cells immediately with $2 \mathrm{~mL}$ quenching buffer thrice before a final washing step with ice cold 1x PBS. The cells were harvested on ice in $250 \mu$ I RIPA quenching buffer using a cell scraper, frozen in liquid $\mathrm{N}_{2}$ and stored at $-80{ }^{\circ} \mathrm{C}$ until proceeding with cell lysis and enrichment of biotinylated proteins.

\subsubsection{Quantitative RT-PCR}

For quantitative real-time PCR, NRCMs were transduced with adenoviral particles APEX2PLN or APEX2-PLNA(1-29) for $48 \mathrm{~h}$ as described above. Subsequently, cells were harvested in 1x PBS using a cell scraper, pelleted and lysed using the QIAShredder homogenizer (Qiagen), and total RNA was isolated by using the RNeasy Mini Kit (Qiagen) according the manufactures protocol. RNA was reverse transcribed by qScript cDNA Super Mix (Quanta Biosciences) following the incubation protocol in Table 5. Quantitative real-time (qRT)-PCR was conducted in triplicate in 96-well plates (Bio-Rad) using the PerfeCTa SYBR Green Supermix (Quanta Biosciences) and the CFX96 (Bio-Rad) with the reported cycles ( Table 6). For relative quantifications, a mix of all samples was diluted 1:10 in multiple steps. Relative mRNA transcript levels (normalized to GAPDH) were calculated using the Bio-Rad 
CFX Manager software version 3.1 and the CT (cycle number) method and are reported as fold change. The primers used are listed in Table 7.

Table 5 First strand cDNA synthesis

\begin{tabular}{lll} 
& Temperature & Time \\
\hline Pre-incubation & $25^{\circ} \mathrm{C}$ & $5 \mathrm{~min}$ \\
cDNA synthesis & $42^{\circ} \mathrm{C}$ & $30 \mathrm{~min}$ \\
Inactivation & $85^{\circ} \mathrm{C}$ & $5 \mathrm{~min}$ \\
End & $4^{\circ} \mathrm{C}$ & hold
\end{tabular}

Table 6 qRT-PCR cycles

\begin{tabular}{lll} 
& Temperature & Time \\
\hline Preincubation & $95^{\circ} \mathrm{C}$ & $60 \mathrm{sec}$ \\
Denaturation & $95^{\circ} \mathrm{C}$ & $10 \mathrm{sec}$ \\
\hline Annealing & $60^{\circ} \mathrm{C}$ & $30 \mathrm{sec}$ \\
Number of cycles & $39 \mathrm{x}$ & \\
Melt curve & $65^{\circ} \mathrm{C}-95^{\circ} \mathrm{C}$ & $0.5^{\circ} \mathrm{C} / 5 \mathrm{sec}$ \\
End & $4{ }^{\circ} \mathrm{C}$ & hold
\end{tabular}

Table 7 qRT-PCR primer

$\begin{array}{ll}\text { Gene } & \text { Primer sequence } \\ \text { EGFP fwd } & \text { 5'-AGAACGGCATCAAGGTGAAC-3' } \\ \text { EGFP rev } & \text { 5'-TGCTCAGGTAGTGGTTGTCG-3' } \\ \text { GAPDH Rat fwd } & \text { 5'-AAGGTCATCCCAGAGCTGAA-3' } \\ \text { GAPDH Rat rev } & \text { 5'-AGGAATGGGAGTTGCTGTTG-3' }\end{array}$

\subsubsection{Cell lysis, enrichment and elution of biotinylated proteins}

For cell lysis, samples were thawed on ice disrupted with 15 strokes through a 27 gauge needle and cleared by centrifugation at $13000 \times \mathrm{g}$ for $10 \mathrm{~min}$ at $4{ }^{\circ} \mathrm{C}$. Protein concentration of the supernatant was determined using the Pierce $660 \mathrm{~nm}$ Protein Assay Kit (Thermo Fisher Scientific). For the ratiometric APEX2 proximity labeling approach, lysates of heavy, medium and light labeled NRCMs were mixed in a ratio of $1: 1: 1$ for a total of $250 \mu \mathrm{g}$ of protein. Biotinylated proteins were enriched by an avidin pulldown assay (Thermo Scientific). For that, $80 \mu \mathrm{l}$ of slurry monomeric avidin beads (Thermo Fisher Scientific) were loaded into a spin column and equilibrated twice in $500 \mu$ I RIPA quenching buffer. The supernatant was removed 
by centrifugation at $100 \times \mathrm{g}$ for $30 \mathrm{sec}$ at $4^{\circ} \mathrm{C}$. $250 \mu \mathrm{g}$ of cleared lysate was added to the monomeric avidin and incubated for $1 \mathrm{~h}$ at $4{ }^{\circ} \mathrm{C}$ with rotation. Avidin beads with bound biotinylated proteins were washed twice with $500 \mu$ RIPA quenching buffer, once with $2 \mathrm{M}$ urea washing buffer followed by two washing steps with RIPA quenching buffer. Further, columns were dried for 2 min by centrifugation at $2000 \times$ g. Finally, biotinylated proteins were eluted in $75 \mu$ l elution buffer for $15 \mathrm{~min}$ at room temperature and $15 \mathrm{~min}$ at $70{ }^{\circ} \mathrm{C}$. The elution fraction was collected by centrifugation at $100 \times \mathrm{g}$ for $1 \mathrm{~min}$ at $4{ }^{\circ} \mathrm{C}$ and supplemented with $25 \mu \mathrm{l}$ of $4 \mathrm{x}$ SDS sample buffer. For ratiometric APEX2 proximity labelling in NRCMs, eluted proteins were analyzed by mass spectrometry (LC-MS/MS).

\subsubsection{Transverse aortic constriction (TAC) to generate pressure overload in mice}

To induce aortic pressure overload, the transverse aorta was constricted by a minimally invasive surgery as described previously ${ }^{55}$. In surgery, mice were anesthetized with $1.5 \%$ isoflurane in $\mathrm{O}_{2}$. A horizontal incision at the jugulum was made to display the transverse aorta. For the constriction, a 27 gauge needle was tied against the aorta and bended with a 5-0 nonabsorbable suture. The 27 gauge needle was removed and the skin closed with a 6-0 suture. After surgery, the mice were placed on a heating plate $\left(37^{\circ} \mathrm{C}\right)$ and monitored to full recovery from isoflurane anesthesia. Sham operated mice underwent the same procedure, only without constriction of the transverse aorta. After surgery, buprenorphine $(0.1 \mathrm{mg} / \mathrm{kg})$ and carprofen (5 $\mathrm{mg} / \mathrm{kg}$ ) pain medication was given subcutaneously the following days. During the postoperative recovery, the health status and the body weight was monitored daily. 1 or 4 weeks after TAC operation, hearts were collected for ventricular cardiomyocyte isolation or ventricular tissue sampling.

\subsubsection{Isolation of adult mouse ventricular cardiomyocytes}

Isolation of adult mouse ventricular cardiomyocytes was performed according to Wagner et al. ${ }^{56}$. Briefly, adult mice (between 12 and 14 weeks) were anesthetized with $3 \%$ isoflurane in $\mathrm{O}_{2}$ and sacrificed by cervical dislocation. Hearts were rapidly extracted, and the proximal aorta was connected to a 21 gauge cannula. Using a modified Langendorff perfusion setup ${ }^{57}$, hearts were perfused with nominally $\mathrm{Ca}^{2+}$ free perfusion buffer for 4 min at $37^{\circ} \mathrm{C}$ and a flow rate of $4 \mathrm{~mL} / \mathrm{min}$. Next, perfusion buffer was exchanged to collagenase type II containing perfusion buffer $\left(600 \mathrm{U} / \mathrm{mL}\right.$, Worthington Biochemical Corporation) for $9 \mathrm{~min}$ at $37^{\circ} \mathrm{C}$. The ventricles were separated from the atria, gently minced in $2 \mathrm{~mL}$ collagenase containing perfusion buffer and triturated by $5 \mathrm{~mL}$ pipette to suspend the cells in solution. Digestion was stopped in $3 \mathrm{~mL}$ 
stopping buffer. Isolated ventricular cardiomyocytes (VCM) were carefully washed three times with stopping buffer at room temperature and resuspended before plating on laminin coated cover slides for immunofluorescence staining. For WB analysis and membrane preparations, cells were washed once in $1 \times$ PBS and pelleted by gravity for 8 min at RT directly after isolation. Cell pellets were snap-frozen in liq. $\mathrm{N}_{2}$ and stored at $-80^{\circ} \mathrm{C}$ until further experiments.

\subsubsection{Sample preparation of mouse heart tissue for biochemistry}

For sampling of ventricular tissue, heart extraction and connection to the Langendorff perfusion setup was conducted as described above. Hearts were perfused with isotonic $\mathrm{NaCl}$ buffer with a flow rate of $4 \mathrm{~mL} / \mathrm{min}$ for $2 \mathrm{~min}$ at $37^{\circ} \mathrm{C}$. Atria were separated from ventricles, which were again separated in left and right ventricle under a binocular microscope. Tissues were snapfrozen in liq. $\mathrm{N}_{2}$ and stored at $-80^{\circ} \mathrm{C}$ until further experiments.

\subsubsection{Sample preparation for confocal and STED immunofluorescence imaging}

For immunofluorescence imaging, isolated ventricular cardiomyocytes were plated on glass cover slides ( $\varnothing 18 \mathrm{~mm}$, width $1.5 \mathrm{~mm}$, Menzel) coated with $2 \mu$ laminin $(2 \mathrm{mg} / \mathrm{mL}$ ) in perfusion buffer. After $30 \mathrm{~min}$ of sedimentation, cells were attached to the glass surface. The perfusion buffer was aspirated, and cells were immediately fixed with $4 \%$ paraformaldehyde (PFA, Sigma) in PBS for 5 min at room temperature.

HEK293A cells or NRCM were seeded with a density of $1 \times 10^{5}$ cells on collagen or $2.5 \times 10^{5}$ cells on laminin $(2 \mathrm{mg} / \mathrm{mL}$ ) coated cover slides ( $\varnothing 18 \mathrm{~mm}$, width $1.5 \mathrm{~mm}$, Menzel), respectively. Cells were cultivated in the respective cell culture medium under humidified conditions with $5 \% \mathrm{CO}_{2}$ at $37^{\circ} \mathrm{C}$ (Heracell VIOS 250i, Thermo Fisher). After cultivation, cells were washed once with $1 \times$ PBS and fixed with 4\% PFA for 5 min at room temperature.

For immunostaining of iPSC derived cardiomyocytes (iPSC-CM), cultivated cells were detached using $0.25 \%$ trypsin/EDTA (Thermo Fisher), counted and reseeded on Matrigel coated cover slides ( $\varnothing 18 \mathrm{~mm}$, width $1.5 \mathrm{~mm}$, Menzel) in cardio recovery medium and cultivated for at least 7 days in cardio culture medium under humidified conditions with $5 \% \mathrm{CO}_{2}$ at $37^{\circ} \mathrm{C}$ (Heracell VIOS 250i, Thermo Fisher). For fixation, cells were washed with 1x PBS and fixed in 4\% Roti-Histofix (Carl Roth) for $20 \mathrm{~min}$ at room temperature.

After fixation, cells were permeabilized and blocked in IF blocking buffer for at least $2 \mathrm{~h}$ at room temperature. Primary antibodies were diluted in $500 \mu \mathrm{l}$ blocking buffer and applied to the cells for overnight incubation at $4^{\circ} \mathrm{C}$. The following day, cells were washed twice in blocking buffer. Anti-mouse or anti-rabbit secondary antibodies were diluted 1:1000 in $500 \mu \mathrm{l}$ IF blocking buffer 
and applied overnight at $4{ }^{\circ} \mathrm{C}$ in the dark. For confocal immunofluorescence microscopy, Alexa Fluor 633 and Alexa Fluor 514 conjugated secondary antibodies were used. For STED immunofluorescence microscopy, STAR 635P and STAR580 conjugated secondary antibodies were used. Excess secondary antibodies were washed away twice with $1 \times$ PBS for $5 \mathrm{~min}$ and again for $10 \mathrm{~min}$. The cover slides with the immunostained cells were embedded in mounting medium (ProLong Gold Antifade Mountant, Thermo Fisher) upside down on a glass objective and dried for overnight at room temperature in the dark. The next day, the edges of the cover slides were sealed with clear nail polish.

\subsubsection{Histology of ventricular heart tissue}

For immunofluorescence staining, ventricular tissue from PLNWT and KO mice were perfused for 2 min with isotonic $\mathrm{NaCl}$ buffer and subsequently fixed in 4\% PFA overnight. The fixed tissue was embedded in paraffin, microtome sliced into $4 \mu \mathrm{m}$ thick histological sections and plated on glass objectives. Prior to immunofluorescence staining, the heart sections were deparaffinized and rehydrated gradually (Table 8). Antigens were unmasked using a $10 \mathrm{mM}$ sodium-citrate buffer for $40 \mathrm{~min}$ at $99^{\circ} \mathrm{C}$. For further incubation steps, the heart sections were encircled with an oil pen to allow the application of small volumes. For blocking and permeabilization, samples were incubated with $400 \mu \mathrm{l}$ 4\% BSA in PBS and $0.1 \%$ Triton-X100 for $1 \mathrm{~h}$ at room temperature. Primary antibodies were diluted in $400 \mu \mathrm{l} \%$ BSA in PBS and applied overnight at room temperature. The next day, samples were washed three times for 5 min in 1X PBS and incubated in anti-mouse or anti-rabbit secondary antibodies diluted 1:300 in $400 \mu \mathrm{l} 4 \% \mathrm{BSA}$ in PBS for $2 \mathrm{~h}$ at room temperature. For STED immunofluorescence microscopy, STAR 635P and STAR580 conjugated secondary antibodies were used. Secondary antibodies were removed by washing three times for $5 \mathrm{~min}$ in $1 \mathrm{x}$ PBS. Heart sections were embedded in mounting medium (ProLong Gold Antifade Mountant, Thermo Fisher) on a glass cover slide and dried for overnight at room temperature in the dark. The next day, the edges of the cover slides were sealed with clear nail polish. 
Table 8 Deparaffinization and rehydration of paraffin embedded histological sections

\begin{tabular}{ll} 
Reagent & Time \\
\hline Xylol & $2 \times 7 \mathrm{~min}$ \\
\hline EtOH 100\% & $2 \times 5 \mathrm{~min}$ \\
\hline EtOH 90\% & $2 \times 5 \mathrm{~min}$ \\
\hline EtOH 70\% & $5 \mathrm{~min}$ \\
\hline EtOH 50\% & $5 \mathrm{~min}$ \\
\hline EtOH 30\% & $5 \mathrm{~min}$ \\
\hline $\mathrm{dH}_{2} \mathrm{O}$ & $5 \mathrm{~min}$ \\
\hline
\end{tabular}

\subsubsection{Confocal microscopy}

Confocal images were acquired with a Zeiss LSM 710 and LSM 880 using a Plan-Apochromat $63 \times / 1.40$ ail objective and a pixel size of $50 \times 50 \mathrm{~nm}$. Alexa Fluor 514 and Alexa Fluor 633 excitation was set to $514 \mathrm{~nm}$ and $633 \mathrm{~nm}$ and fluorophore emission was detected between 520-620 nm and 640-740 nm, respectively. Confocal laser power was adjusted to maximize resolution following established protocols ${ }^{56}$. Raw images were processed with Fiji software (https://imagej.net/Fiji) using background subtraction (rolling ball radius: 50 ), local contrast enhancement, and smoothing (Gaussian, sigma 1 pixel).

\subsubsection{Immunofluorescence STED nanoscopy}

STED images were acquired with a Leica TCS SP8 system with a HC PL APO C2S 100x/1.40 oil objective and a pixel size of $16.23 \times 16.23 \mathrm{~nm}$. STAR 635P and STAR 580 fluorophore excitation was set to $635 \mathrm{~nm}$ and $580 \mathrm{~nm}$ and fluorophore emission was detected between 650-700 nm and 600-630 nm, respectively. For STED depletion of STAR 580 and STAR 635P, a $775 \mathrm{~nm}$ laser beam was used. Raw images were processed in Fiji (https://imagej.net/Fiji) using background subtraction (rolling ball radius: 50 ), local contrast enhancement, and smoothing (Gaussian, sigma 1 pixel).

\subsubsection{Membrane enrichment from isolated ventricular cardiomyocytes}

Frozen isolated ventricular cardiomyocytes were thawed on ice and resuspended in detergentfree homogenization buffer $(0.1 \mathrm{~g}$ cells $/ 1 \mathrm{~mL}$ homogenization buffer). The cardiomyocytes were disrupted with a $2 \mathrm{~mL}$ potter homogenizer (RW20 digital, IKA) for 100 strokes at $2000 \mathrm{rpm}$ on ice. The homogenate was centrifuged at $400 \mathrm{xg}$ for $10 \mathrm{~min}$ at $4{ }^{\circ} \mathrm{C}$ (HARAEUS Fresco21 
centrifuge, Thermo Fisher). The resulting pellet was potter homogenized again in $1 \mathrm{~mL}$ homogenization buffer with 50 strokes and repeatedly centrifuged. The cleared lysates were combined and centrifuged at $3000 \mathrm{xg}$ for $10 \mathrm{~min}$ at $4{ }^{\circ} \mathrm{C}$ to remove nuclei and cell debris. The perinuclear fraction (PNF) was differential centrifuged at $9000 \times \mathrm{g}$, at $15000 \times \mathrm{g}$ for $20 \mathrm{~min}$ at $4{ }^{\circ} \mathrm{C}$, or ultracentrifuged at $100000 \times \mathrm{g}$ for $1 \mathrm{~h}$ at $4{ }^{\circ} \mathrm{C}$ (MLS50 swingout rotor; Optima MAX-XP Ultracentrifuge, Beckman Coulter). The resulting pellets were resuspended in homogenization buffer and the protein concentration was determined by Pierce BCA-Protein Assay (Thermo Fisher). For immunoblotting, the collected supernatants and resuspended pellets were prepared with a final $1 x$ NuPAGE LDS sample buffer (4x, Thermo Fisher). For blue native gel electrophoresis, $50 \mu \mathrm{g}$ or $100 \mu \mathrm{g}$ of resuspended membranes were aliquoted and pelleted at $15000 \mathrm{x} g$ for $20 \mathrm{~min}$ or $100000 \mathrm{xg}$ for $1 \mathrm{~h}$ at $4^{\circ} \mathrm{C}$ (MLA-150 fixed rotor, Beckman Coulter).

\subsubsection{Membrane solubilization for blue native gel electrophoresis}

Enriched membrane fractions were solubilized in solubilization buffer $A$ and solubilized with $6 \mathrm{~g}$ per gram protein for $30 \mathrm{~min}$ on ice. Un-solubilized membranes were pelleted with $21000 \mathrm{xg}$ for $20 \mathrm{~min}$ at $4^{\circ} \mathrm{C}$. The supernatant was supplemented with $1: 10(\mathrm{v} / \mathrm{v}) 50 \%$ glycerol and 1:10 $(\mathrm{v} / \mathrm{v})$ coomassie blue solution. $50 \mu \mathrm{g}$ or $100 \mu \mathrm{g}$ of membranes were loaded on blue native gels for gel electrophoresis.

\subsubsection{Blue native gel electrophoresis}

Blue native gel electrophoresis (BN-PAGE) was conducted as published by Wittig, Braun and Schägger ${ }^{58}$. Enriched membranes were solubilized as described above. For BN-PAGE the PROTEAN II xi (Biorad) was used. For the separation of cardiac membranes, a tricine-imidazol gel with a $4-13 \%$ continuous gradient was cast with a gradient mixer $(16 \times 20 \mathrm{~cm})$ and stored overnight at $4{ }^{\circ} \mathrm{C}$ to allow polymerization. The next day, a stacking gel was cast, chamber and gel was assembled, and filled with $1000 \mathrm{~mL}$ anode buffer and $300 \mathrm{~mL}$ cathode buffer B. Solubilized membranes were applied together with a native molecular weight marker (SERVA) and separated at $100 \mathrm{~V}$ for $20 \mathrm{~min}$ followed by $400 \mathrm{~V}$. When the blue running front reached one third of the gel the cathode buffer was change to $B / 10$. The BN gel was further separated at $400 \mathrm{~V}$ until the blue front reached the end. After the run, the BN gel was equilibrated in $\mathrm{dH}_{2} \mathrm{O}$. For complexome profiling single gel lanes were cut in 60 equal slices, each slice further into smaller pieces and transferred into $0.5 \mathrm{~mL}$ reaction tubes with $200 \mu \mathrm{ld} \mathrm{H}_{2} \mathrm{O}$. The gel pieces were subjected to tryptic digestion and mass spectrometry analysis as described below.

For immunoblotting, the BN gel was equilibrated in transfer buffer and proteins were transferred onto a PVDF membrane at $100 \mathrm{~V}$ for $2 \mathrm{~h}$ at $4{ }^{\circ} \mathrm{C}$. After blotting the PVDF membrane 
was destained with pure methanol and subjected to blocking in 5\% milk in TBS-T. The primary and secondary antibody incubations were conducted as described under 'Complexome profiling and nanoLC-MS/MS analysis'.

\subsubsection{D BNE/SDS-PAGE}

Enriched membranes from isolated mouse ventricular cardiomyocytes were prepared as described under 'Membrane solubilization for blue native gel electrophoresis'. Briefly, $50 \mu \mathrm{g}$ of total membranes were solubilized with $6 \mathrm{~g}$ digitonin $/ \mathrm{g}$ protein for $45 \mathrm{~min}$ at $4{ }^{\circ} \mathrm{C}$ and cleared for $20 \mathrm{~min}$ at $20000 \mathrm{xg}$ at $4{ }^{\circ} \mathrm{C}$. The supernatant was supplemented with 1:10 coomassie blue solution and 1:10 [50\%] glycerol. The solubilized membranes were applied on a Native PAGE $3-12 \%$ Bis-Tris Protein gel (Thermo Fisher) and separated at $150 \mathrm{~V}$ for $60 \mathrm{~min}$ in 'dark blue' cathode buffer (Thermo Fisher) and at $250 \mathrm{~V}$ for additional $60 \mathrm{~min}$ using 'light blue' cathode buffer (Thermo Fisher). The electrophoresis was carried out at $4{ }^{\circ} \mathrm{C}$. After the run BN-gel lanes were subjected to immunoblotting or separated in the second dimension on a $4-20 \%$ TrisGlycine gel (2D-well, Thermo Fisher). Before that, the BN-gel lane was equilibrated in 1\% SDS, diluted in $\mathrm{dH}_{2} \mathrm{O}$, for $30 \mathrm{~min}$ at room temperature. The $\mathrm{BN}$-gel lane was cut to size, subjected to the 2D-well, and separated at constant $125 \mathrm{~V}$ for $90 \mathrm{~min}$ at room temperature. After the membranes were separated in the second dimension, the SDS-gel was subjected to immunoblotting on PVDF membranes at $100 \mathrm{~V}$ for $2 \mathrm{~h}$ at $4{ }^{\circ} \mathrm{C}$, as described under 'Immunoblotting'.

\subsubsection{Mass spectrometry}

Mass spectrometry analyses were performed by Dr. Christof Lenz, Prof. Henning Urlaub and the Proteomics Service Unit (University Medical Center Göttingen).

\subsubsection{APEX2 proximity labeling and nanoLC-MS/MS analysis for quantitative mass spectrometry}

Samples were reconstituted in 1x SDS sample buffer and separated on 4-12 \% Bis-Tris minigels (NuPAGE Novex, Thermo Fisher) using half of the gel length. Gels were stained with Coomassie Blue in order to separate individual protein lanes. Each lane was cut into 11 equal pieces. Gel slices were washed, reduced with $10 \mathrm{mM}$ dithiothreitol (DTT, Sigma-Aldrich), alkylated with $55 \mathrm{mM}$ 2-iodoacetamide (Sigma-Aldrich), and trypsin-digested with the (sequencing grade, Promega) overnight. After trypsin digestion, peptide mixtures were extracted, dried in a SpeedVac (Eppendorf), reconstituted in $2 \%$ acetonitrile/ $0.1 \%$ formic acid/ 
(v:v) and prepared for nanoLC-MS/MS as described previously ${ }^{59}$.

For mass spectrometric analysis samples were enriched on a self-packed reversed phaseC18 precolumn and separated on an analytical reversed phase-C18 column using a $30 \mathrm{~min}$ linear gradient of 5-35\% acetonitrile/0.1\% formic acid (v:v) at $300 \mathrm{nl} \mathrm{min}{ }^{-1}$. The eluent was analyzed on a Q Exactive HF-X hybrid quadrupole/orbitrap mass spectrometer (ThermoFisher Scientific, Dreieich, Germany) equipped with a Flexlon nanoSpray source and operated under Excalibur 2.5 software using a data-dependent acquisition method. Each experimental cycle was of the following form: one full MS scan across the 350-1600 m/z range was acquired at a resolution setting of 70,000 FWHM, and AGC target of $1 * 10 \mathrm{e} 6$ and a maximum fill time of 60 ms. Up to the 12 most abundant peptide precursors of charge states 2 to 5 above a $2^{*} 10^{4}$ intensity threshold were then sequentially isolated at 2.0 FWHM isolation width, fragmented with nitrogen at a normalized collision energy setting of $25 \%$, and the resulting product ion spectra recorded at a resolution setting of 17,500 FWHM, and AGC target of 2*10e5 and a maximum fill time of $60 \mathrm{~ms}$. Selected precursor $\mathrm{m} / \mathrm{z}$ values were then excluded for the following $15 \mathrm{~s}$. Two technical replicates per sample were acquired.

\subsubsection{APEX2 proximity labeling data processing}

Raw data were processed using MaxQuant Software version 1.5.7.4 (Max Planck Institute for Biochemistry, Martinsried, Germany). Proteins were identified against a UniProtKB-derived rattus norvegicus protein sequence database (v2018.02, 37830 protein entries) along with a set of common lab contaminants. The search was performed with trypsin as enzyme and iodoacetamide as cysteine blocking agent. Up to two missed tryptic cleavages and methionine oxidation as a variable modification were allowed for. Instrument type 'Orbitrap' was selected to adjust for MS acquisition specifics. The Arginine R10, R6 and Lysine K8, K6 labels including the 'Re-quantify' option were specified for relative protein quantitation. For identification of APEX2 biotinylated proteins, the normalized and $\log _{2}$ transformed ratios of APEX2-PLN versus APEX2-PLN $\Delta$ (1-29) or eGFP were calculated by MaxQuant Software. Only proteins identified in all technical and biological replicates were used for analysis. Positive enriched biotinylated proteins were evaluated for statistical significance $p<0.05$ by one sample z-test using Microsoft Excel software. For visualization, scatter plots of APEX2-PLN / APEX2-PLN $\Delta(1-29)$ ratios t on the $\mathrm{X}$-axis and APEX2-PLN / eGFP ratios on the $\mathrm{Y}$-axis were generated with Prism version 7.03 Software (GraphPad). 


\subsubsection{Gene enrichment analysis}

133 APEX2-PLN enriched proteins were enriched for their biological functions using ToppGene Suite tool ToppFun (default setting: FDR correction, $p$ value cut off at 0.05 and gene limit set between and including 1 and 2000 per pathway) ${ }^{60}$.

\subsubsection{Complexome profiling and nanoLC-MS/MS analysis}

Complexome profiling and mass spectrometry are part of and were performed as published by Alsina et al. ${ }^{61}$. A digitonin solubilized, enriched membrane fraction of isolated cardiomyocytes from PLN WT and KO mice were separated on a blue native $4-13 \%$ gradient gel as described ${ }^{58}$. Gel lanes were cut in 60 equal pieces.

After washing, gel slices were reduced with $10 \mathrm{mM}$ dithiothreitol (DTT, Sigma-Aldrich), alkylated with $55 \mathrm{mM}$ 2-iodoacetamide (Sigma-Aldrich), and trypsin-digested (sequencing grade, Sigma-Aldrich) overnight. After trypsin,digestion, peptide mixtures were then extracted, dried in a SpeedVac (Eppendorf), reconstituted in 2\% acetonitrile/0.05\% TFA acid/ (v:v) and prepared for nanoLC-MS/MS as described previously ${ }^{59}$.

For mass spectrometric analysis samples were enriched on a C18 precolumn (Dionex $5 \mathrm{x}$ $0.3 \mathrm{~mm}$ ID) and separated on an analytical reversed phase-C18 column $(0.075 \mathrm{~mm}$ ID $\times 300$ mm, Reprosil-Pur 120 C18-AQ, $3 \mu \mathrm{m}$, Dr. Maisch) using a 46 min linear gradient of $5-40 \%$ buffer B (80\% acetonitrile/0.08\% formic acid (v:v)) at $300 \mathrm{nl}$ min-1). The eluent was analyzed on a Q Exactive HF-X hybrid quadrupole/orbitrap mass spectrometer (ThermoFisher Scientific, Dreieich, Germany) equipped with a Flexlon nanoSpray source and operated under Xcalibur 4.1.31.9 software using a data-dependent acquisition method. Each experimental cycle was of the following form: one full MS scan across the 350-1600 m/z range was acquired at a resolution setting of 60,000 FWHM, and AGC target of $1 * 10 \mathrm{e} 6$ and a maximum fill time of 50 ms. Up to the 30 most abundant peptide precursors of charge states 2 to 6 above a $2{ }^{*} 10 \mathrm{e} 4$ intensity threshold were then sequentially isolated at 1.4 FWHM isolation width, fragmented with nitrogen at a normalized collision energy setting of $30 \%$, and the resulting product ion spectra recorded at a resolution setting of $15,000 \mathrm{FWHM}$, an AGC target of $2 * 10 \mathrm{e} 5$ and a maximum fill time of $54 \mathrm{~ms}$. Selected precursor $\mathrm{m} / \mathrm{z}$ values were then excluded for the following 20 s. Two technical replicates per sample were acquired.

\subsubsection{Complexome profiling data processing}

All raw data were processed using MaxQuant Software version 1.6.0.1 (Max Planck Institute for Biochemistry, Martinsried, Germany). Proteins were identified against a UniProtKB-derived Mus musculus protein sequence database (v2016.04) along with a set of common lab 
contaminants. The search was performed with trypsin as enzyme and iodoacetamide as cysteine blocking agent. Up to two missed tryptic cleavages and methionine oxidation as a variable modification were allowed for. Instrument type 'Orbitrap' was selected to adjust for MS acquisition specifics. Two technical replicates were measured and averaged, and protein abundance migration profiles were normalized considering multiple profiles, that is, taking into account intensity values from all slices. Each protein migration profile was maximum normalized, over PLN WT and KO, and colored in the given look up table using Microsoft Excel software. Data cluster analysis was performed by cluster 3.0 software ${ }^{62}$ with average linkage and uncentered Pearson correlation.

\subsubsection{Statistical analysis}

Data were represented as mean \pm standard error of the mean (SEM). The unpaired two-tailed students t-test was used, considering a p-value of less than 0.05 as statistically significant. For APEX2 proximity labeling data analysis, the $z$-test was used testing against ' 0 ' and a $p$-value of less than 0.05 was considered as statistically significant. 


\section{Identification of novel phospholamban protein interactions by complexome profiling}

\subsection{Introduction}

Proteins are regulators and effectors involved in all cellular processes. Through protein-protein interactions they can elicit multiple and complex regulatory functions that depend on their interactions, and organization in protein complexes. Depending on the stability and environment of a specific complex, its protein components can be isolated and characterized by classical biochemical methods, i.e. by affinity purification ${ }^{63}$. More difficult is the characterization of rare complexes, mainly based on transient or labile protein-protein interactions. Recently, there have been developed several methods for the analysis of labile protein complexes, i.e. yeast-two-hybrid ${ }^{64}$ or proximity-dependent protein labeling in living cells ${ }^{65,66,67}$. One of these methods is the APEX2 proximity labeling ${ }^{54}$ which will be subject to chapter II of this thesis for the identification of novel PLN interacting proteins in cardiomyocytes. Although these methods are successfully applied ${ }^{68-70,71}$, they rely on genetic expression strategies, which change the endogenous environment and potentially the interaction behavior of a protein of interest. A recently developed method to directly identify the components of labile protein-protein interactions is called complexome profiling ${ }^{72,73,74}$. This method is based on subcellular fractionation and extraction under native conditions, while membranes can be analyzed using non-ionic detergents. The 'complexome' of biological membranes, total cell or tissue homogenates can be fractionated by blue native gel electrophoresis ${ }^{75,76,58}$, which provides sufficient separation in the mass range of $10 \mathrm{kDa}$ to 10 $\mathrm{MDa}^{76}$. For complexome profiling, BNE lanes are cut in 60 (or more) even slices, subjected to tryptic digestion, and analyzed by label-free mass spectrometry (Figure 6). More than 100 proteins per gel slice can be detected and quantified at the same time. Since not every protein will have the same abundance in every gel slice, abundance profiles are generated for all detected proteins and visualized by heatmap (Figure 6). Similar migration profiles can be hierarchically clustered ${ }^{77}$, and well-defined protein clusters or complexes are visualized. 
BN-PAGE Gel slices

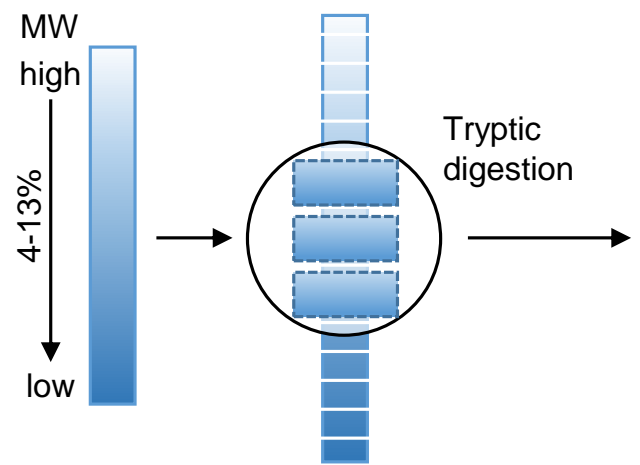

Heat map \& Migration profiles

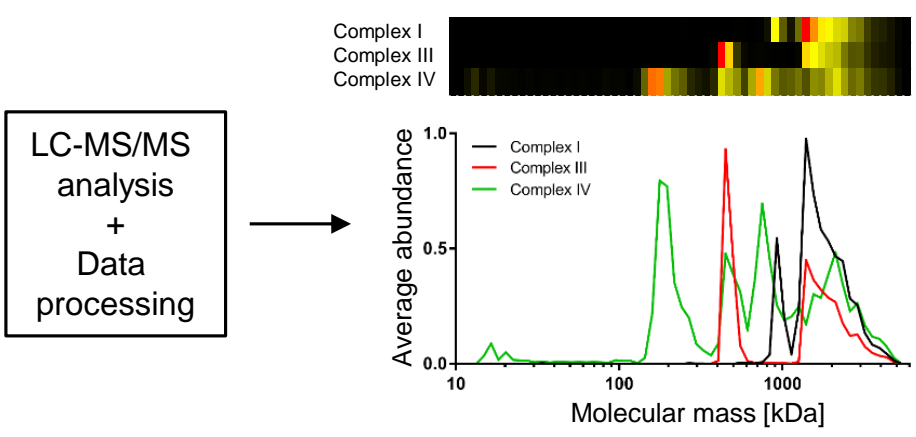

Figure 6 Complexome profiling workflow for cardiac membranes

Isolated membranes from cardiac samples were solubilized and separated by blue native gel electrophoresis (BN-PAGE). Gel lanes are cut in equal slices and subjected to tryptic digestion. Peptides were analyzed by mass spectrometry (LC-MS/MS) and processed using MaxQuant software. Protein intensities are maximum normalized for all slices showing migration profiles as heatmap and line profiles for illustration, exemplary for respiratory chain complex I, III, and IV.

For the analysis of native cardiac protein complexes and potentially novel interaction partners of PLN, total membranes of mouse WT and PLN knockout isolated ventricular cardiomyocytes were subjected to complexome profiling. In contrast to the prevailing models, a conceptually new complex comprised of RyR2 and PLN/SERCA2a has been identified ${ }^{61}$. This work is part of a published manuscript, which identified PPP1R3A as a novel PP1-regulatory subunit within the RyR2 complex in addition to the previously established PLN/SERCA2a complex suggesting a new co-regulatory mechanism.

Also, complexome profiling data revealed by hierarchical cluster analysis the sarcolemmal membrane-associated protein (SLMAP) as a potential novel interacting protein of PLN. SLMAP is a tail-anchored membrane protein initially identified in cardiac membranes ${ }^{78,79}$. The SLMAP gene encodes three major isoforms, which are further transcribed by alternative splicing (Figure 7$)^{78,79}$. SLMAP1 is predominantly expressed in cardiac and slow-twitch muscles, whereas SLMAP3 is expressed ubiquitously, e.g. in heart, brain and skeletal muscle ${ }^{78,79}$. All isoforms commonly share a leucine zipper amphipathic motif involved in dimerization and protein interactions. Furthermore, coiled-coil carboxyl hydrophobic segments are predicted for the cytosolic domain of SLMAPs. Additionally, several putative phosphorylation domains have been identified for different kinases, i.e. casein kinase ${ }{ }^{79}$ or PKC and $\mathrm{PKA}^{78}$. The largest SLMAP3 isoform has a unique N-terminal stretch harboring the forkhead-associated domain (FHA) targeting the protein to centrosomes during embryonic development ${ }^{80}$. All SLMAPs 
express either one of two transmembrane domains (TM1 or TM2) by alternative splicing that may influence subcellular localization ${ }^{81,79}$.

SLMAP has been studied previously in skeletal muscle development and the organization of the excitation-contraction (E-C) coupling apparatus ${ }^{82}$. SLMAP gene mutations have been recently associated with the Brugada syndrome and defective trafficking of the cardiac Nav1.5 channel to the plasma membrane in cardiomyocytes ${ }^{83,84}$. Single SLMAP isoform overexpression in mice showed that SLMAP1 leads to reduced protein expression of RyR2 and SERCA2 $\mathrm{a}^{85}$, and SLMAP3 a regulator of cardiomyocyte contraction is ${ }^{86}$. Still, the precise functional role of SLMAP in the heart needs to be elucidated. This work describes a previously unknown interaction of SLMAP with PLN, the functional implications of which warrant further in-depth investigation given that PLN represents a major phospho-regulatory protein of the ventricles.

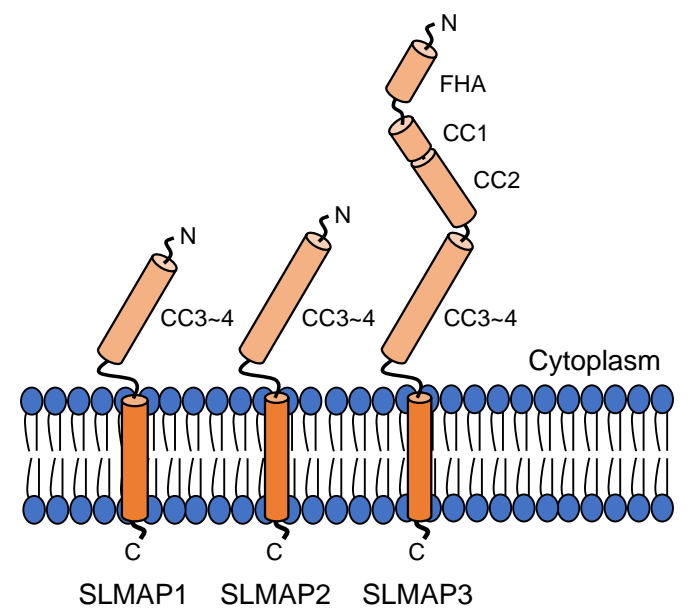

Figure 7 Topology of the sarcolemmal membrane-associated protein SLMAP1-3 isoforms

Three SLMAP isoforms transcribed by alternative splicing are translated into tail-anchored multi-domain membrane proteins. They differ through their coiled-coil cytosolic domains (CC), which facilitate proteinprotein interactions. Additionally, SLMAP3 has a unique N-terminal forkhead-associated domain (FHA). 


\subsection{Results}

\subsubsection{Isolation and solubilization of cardiac membranes for BN-PAGE}

To investigate native protein complexes, membranes from isolated ventricular cardiomyocytes $^{56}$ were enriched. To identify specifically PLN interacting proteins, the enrichment of membranes from the sarcoplasmic reticulum (SR) was intended. Following protocol I (Figure 8A) ${ }^{87}$, a clear separation between the membrane proteins PLN, SERCA2a and CAV3, and the soluble protein GAPDH was observed (Figure 8B). After $15.000 \times \mathrm{g}$ centrifugation, the soluble fraction still contained $S R$ and sarcolemmal $(S L)$ proteins, which lead to protocol II. (Figure $8 \mathrm{~A})$. There, increased speed $(100.000 \times \mathrm{g})$ was used to collect total cardiac membranes (Figure $8 \mathrm{C}$ ). The soluble fraction contained after the centrifugation at $100.000 \times \mathrm{g}$ no detectable SERCA2a and not pelleted pentameric PLN and CAV3. We initially aimed to separate the mitochondria at $9.000 \mathrm{xg}$ and enrich only SR and SL membranes. By immunoblotting, we also detected mitochondrial proteins in the $100.000 \mathrm{xg}$ membrane fraction using VDAC1 as mitochondrial membrane marker. 
A

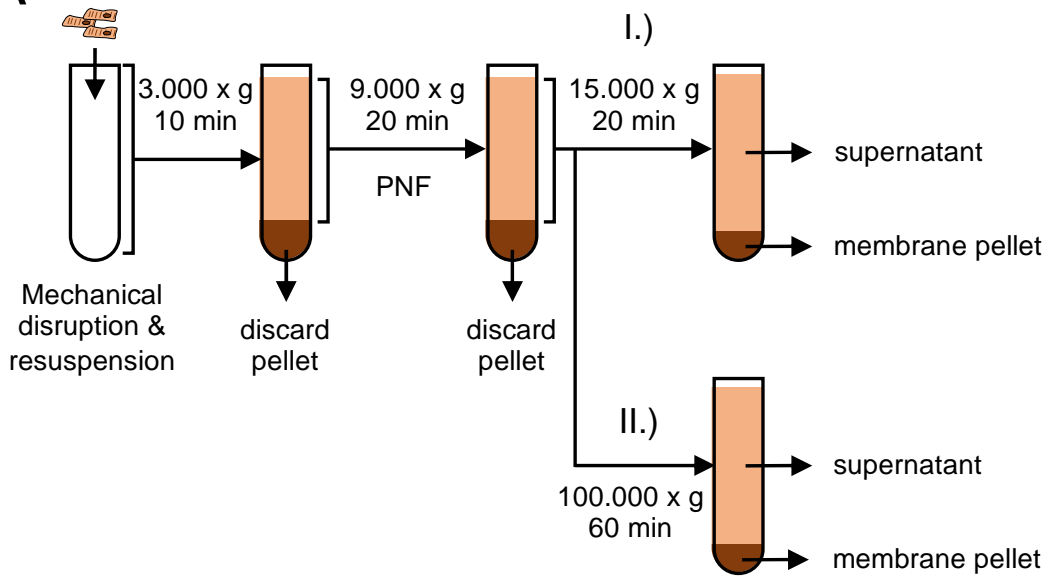

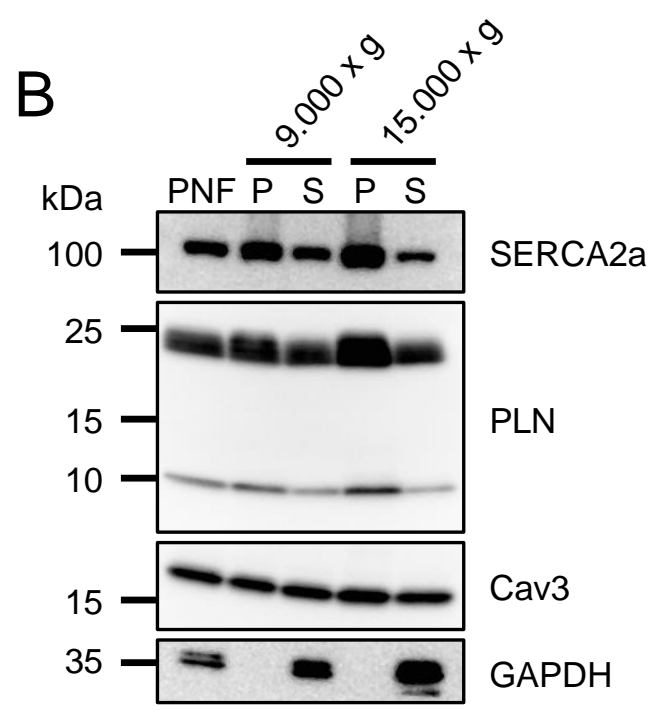

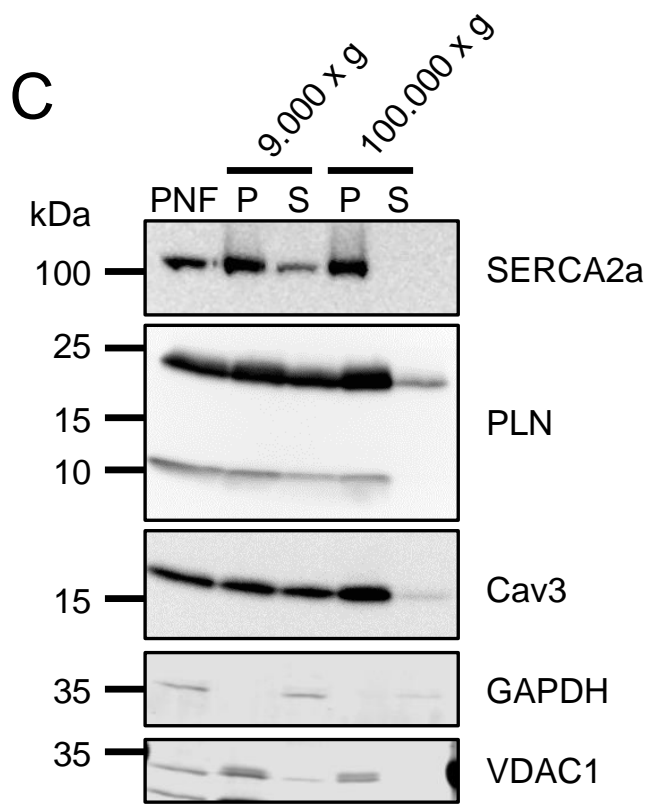

Figure 8 Membrane isolation from mouse ventricular cardiomyocytes

A, Workflow for the separation of sarcoplasmic reticulum membranes by differential centrifugation. After mechanical disruption and resuspension in detergent-free sucrose buffer, cell debris and nuclei were removed at $3.000 \mathrm{xg}$. To remove mitochondria, the peri-nuclear fraction (PNF) was centrifuged at 9.000 $\mathrm{x}$ g. The supernatant was finally centrifuged at I.) $9.000 \mathrm{xg}$ or II.) $100.000 \mathrm{xg}$ to obtain SR membranes. B-C, Immunoblots showing intermediate steps of the enriched membranes by differential centrifugation from isolated ventricular cardiomyocytes following protocol I (B) or II (C).

Limiting by incomplete separation of mitochondrial from SR membranes and loss of SR and $\mathrm{SL}$ membranes at $9.000 \times \mathrm{g}$ (Figure 8B,C), total membrane fractions were used for further analysis (Figure 9A). Cardiomyocytes were lysed in detergent-free sucrose buffer using a potter homogenizer to preserve native protein complexes. In preparation for the blue native 
gel electrophoresis (BN-PAGE), membranes were solubilized (Figure 9B) as follows. To better define the stability and the nature of cardiac protein complexes, two different detergents were tested. Digitonin solubilized membranes showed stronger signals for PLN and SERCA2a after BN-PAGE and immunoblotting compared to n-dodecyl B-D-maltopyranoside (DDM), indicating sufficient solubilization (Figure 9C). Furthermore, PLN and SERCA2a may reside in macromolecular complexes, while only SERCA2a remains both in WT and PLN KO complexes (Figure 9D). Taken together, for the complexome profiling analysis total cardiac membranes of WT and PLN KO ventricular cardiomyocytes were isolated by ultracentrifugation. Digitonin solubilization was found to preserve macromolecular complexes of PLN and SERCA2a. 
A
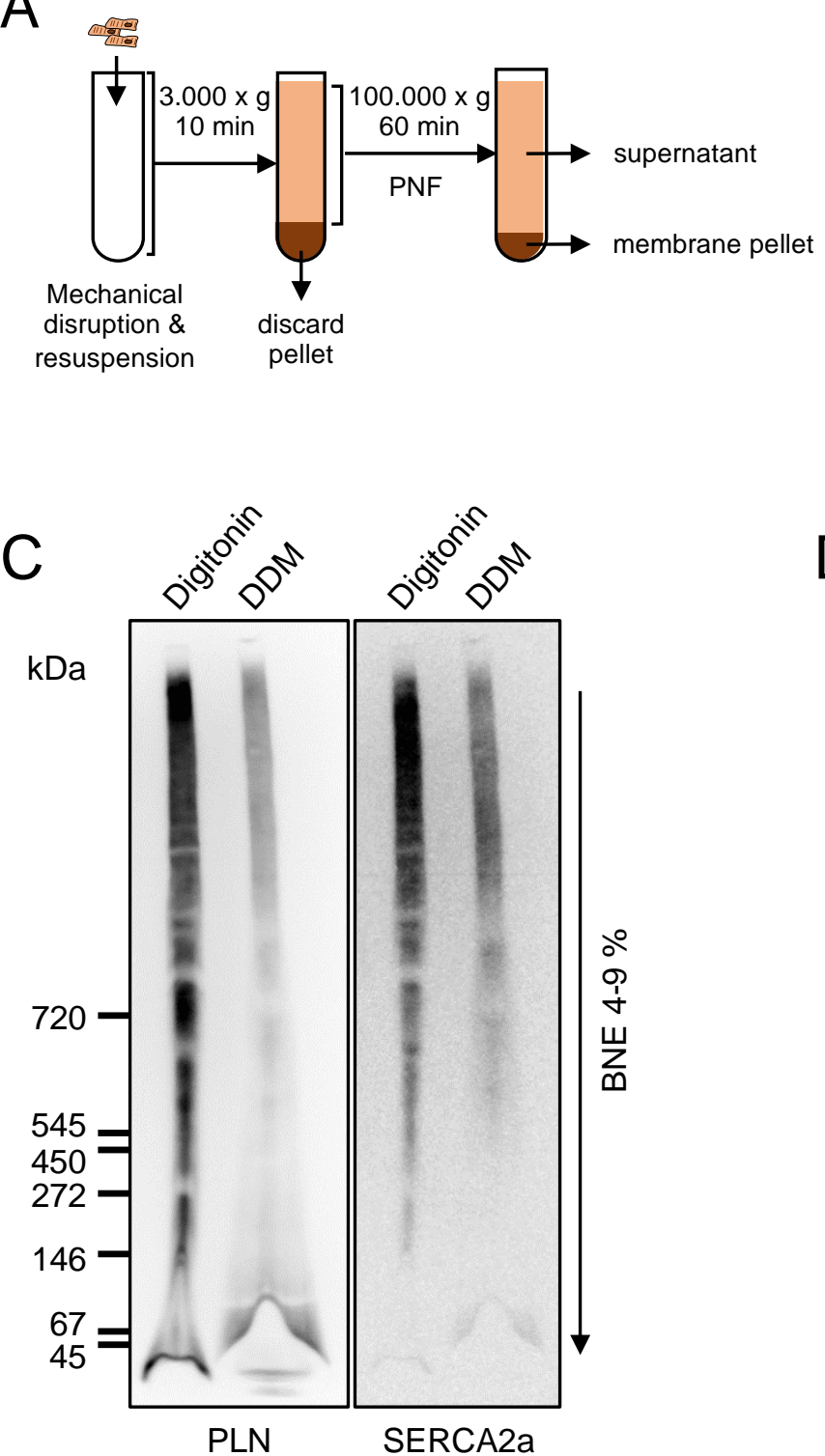

B

membrane

pellet

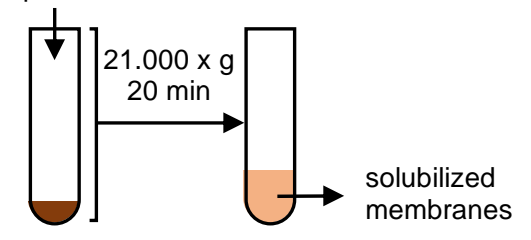

detergent

solubilization

$\mathrm{D}$

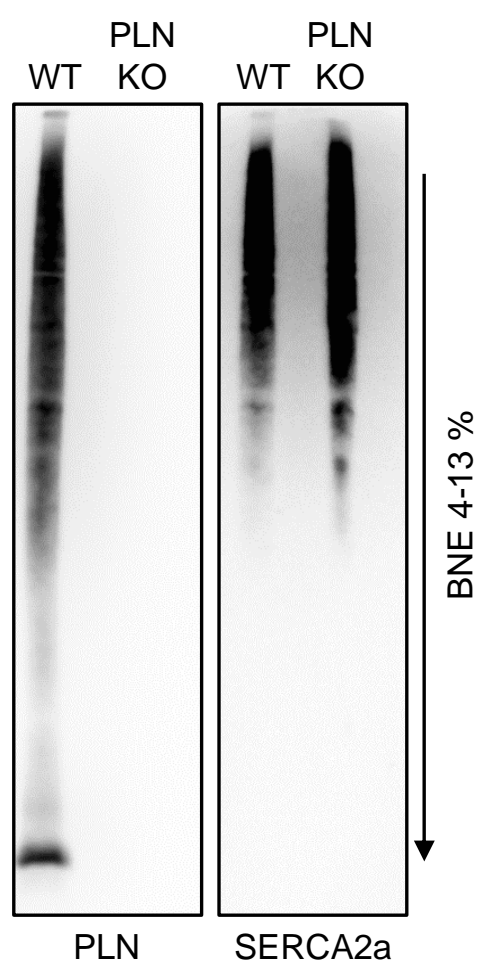

Figure 9 Membrane isolation and solubilization from ventricular cardiomyocytes

A, Workflow of the final membrane isolation protocol for isolated mouse ventricular cardiomyocytes. After mechanical disruption and resuspension in detergent-free sucrose buffer, cell debris and nuclei were removed at $3.000 \mathrm{xg}$. After that, the peri-nuclear fraction $(P N F)$ was centrifuged at $100.000 \times \mathrm{g}$ for $60 \mathrm{~min}$. B, Workflow of the membrane solubilization before BN-PAGE. $100.000 \times \mathrm{g}$ membranes were solubilized with $6 \mathrm{~g}$ digitonin per gram protein. Un-solubilized proteins were removed at $21.000 \mathrm{xg}$. C, Immunoblots of digitonin or n-dodecyl B-D-maltopyranoside (DDM) solubilized ventricular membranes separated by $4-9 \%$ BNE gradient gel showing different signal intensities for PLN and SERCA2a. D, Immunoblots of digitonin solubilized membranes from WT and PLN KO ventricular cardiomyocytes separated by a $4-13 \%$ BNE gradient gel. 


\subsubsection{Complexome profiling analysis of native cardiac membranes}

For complexome profiling, mouse WT and PLN KO ventricular cardiomyocyte (VCM) membranes were digitonin-solubilized and separated by BN-PAGE. Each BN lane was cut in 60 gel slices for in-gel tryptic digestion and subjected to mass spectrometry analysis (LCMS/MS) (Figure 10A). For each detected protein, intensity profiles obtained by MaxQuant software were processed and analyzed. Mass spectrometry sample preparation and data acquisition were carried out by Dr. Christof Lenz, Prof. Henning Urlaub, and the Proteomic Service Unit (University Medical Center Goettingen).

The binding of the anionic dye coomassie to hydrophobic mainly membrane proteins induces a charge shift and allow their migration to the anode. Some but not all soluble proteins have basic amino acid residues and bind coomassie as well ${ }^{88}$. For quality control and mass calibration, soluble and integral membrane proteins, and protein complexes with known molecular weight were assigned to BN-gel slice number of their abundance (Figure 10B,C). This calibration showed a linear separation of soluble and membrane proteins and indicates a good separation of the cardiac membranes. 
A

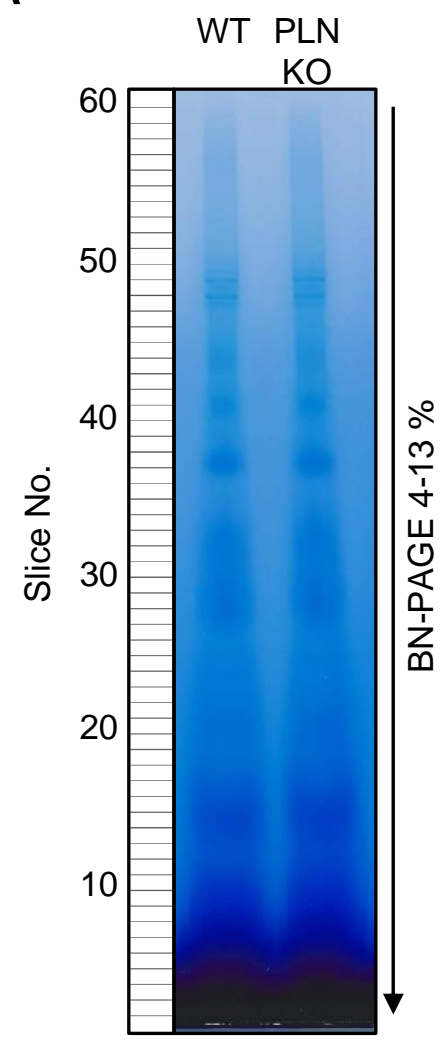

B

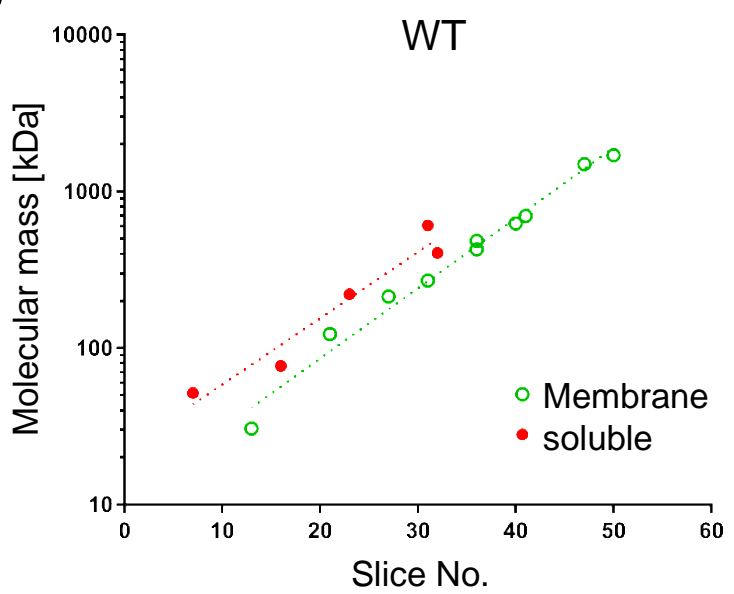

C

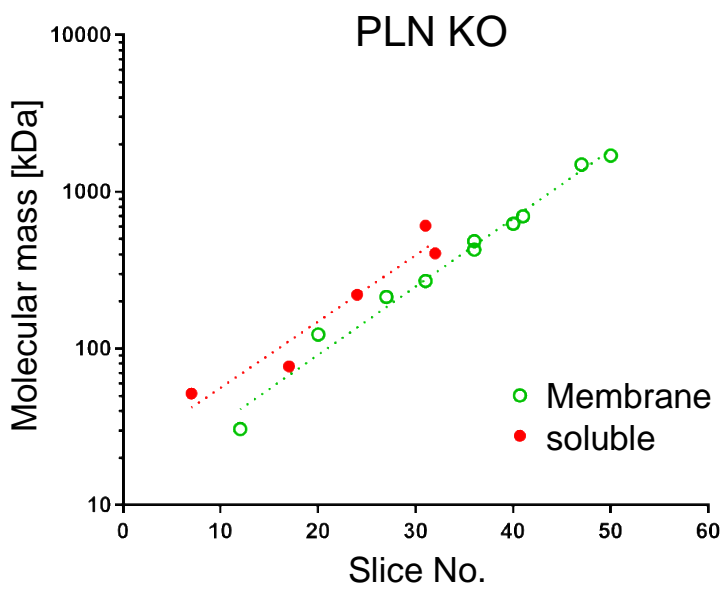

Figure 10 BN-PAGE gel for complexome profiling and mass calibration

A, Blue native gel lanes from WT and PLN KO mouse VCM. Indicated 60 slices were cut and subjected to tryptic in-gel digestion and mass spectrometry. For mass calibration of WT (B) and PLN KO (C), the known molecular weight 88 of soluble and integral membrane proteins and protein complexes were plotted against their slice number. in blue native electrophoresis. Soluble and membrane protein show different migration behavior within the $\mathrm{BN}$-gel based on their hydrophobic surface and affinity for coomassie. Modified from Alsnia et al. ${ }^{61}$

Furthermore, complexome profiling was previously used for the characterization of mitochondrial OXPHOS complexes ${ }^{74,73,89}$. Due to the high abundance of mitochondrial proteins in the total cardiac membrane fraction, mitochondrial protein complexes were used as wellestablished quality control. Heat maps and migration profiles of WT and PLN KO VCM membranes showed the abundance of monomeric complexes as complex II, IV and V. Higher complexes as the dimer of complex III (III $)_{2}$ and multimer of complex IV (IV $\mathrm{n}_{\mathrm{n}}$ as well as supercomplexes with complex I, III and IV were identified. Apparently, PLN KO mitochondrial complexes showed the same distribution as WT suggesting no mitochondrial defects. 


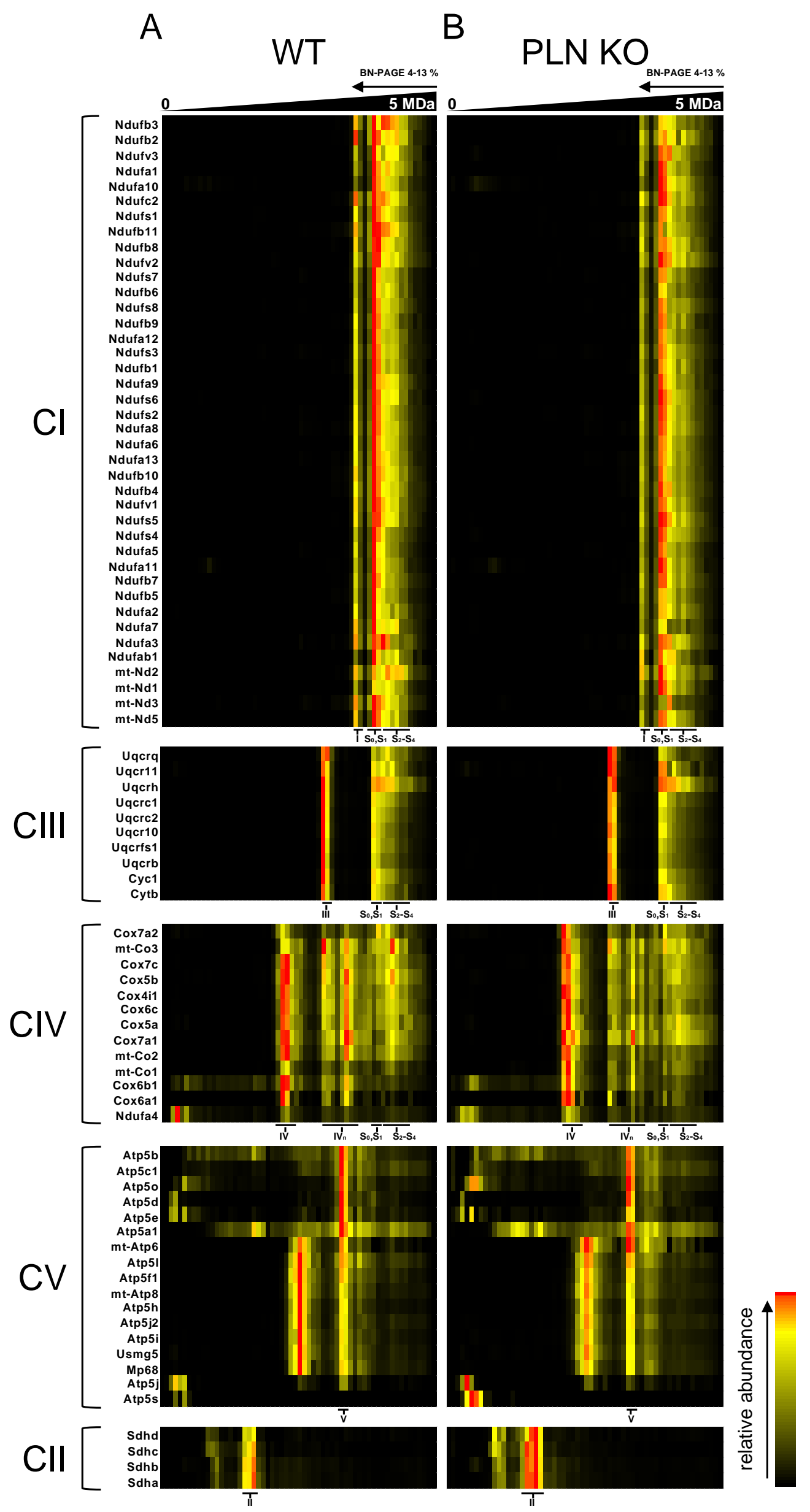


C

WT

BN-PAGE $4-13 \%$
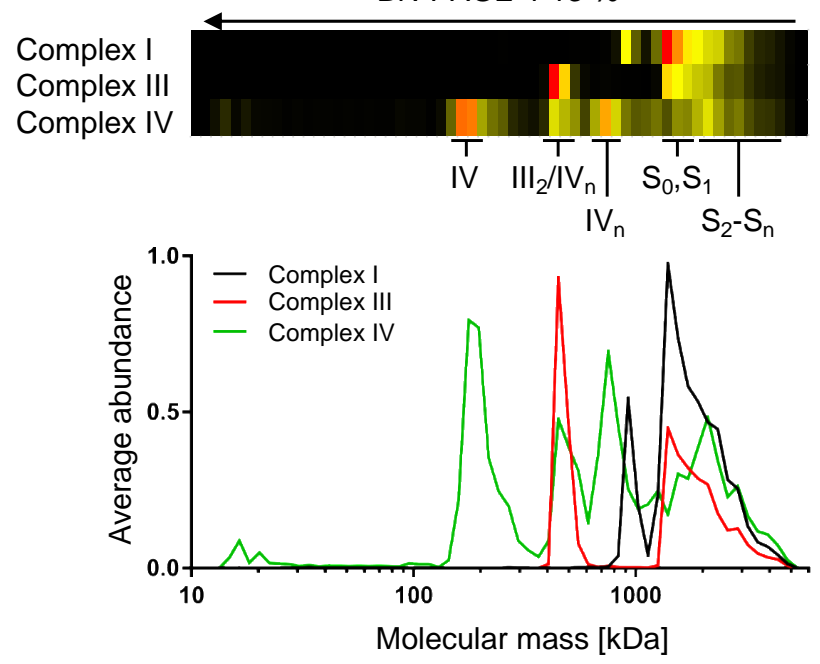

$\mathrm{D}$
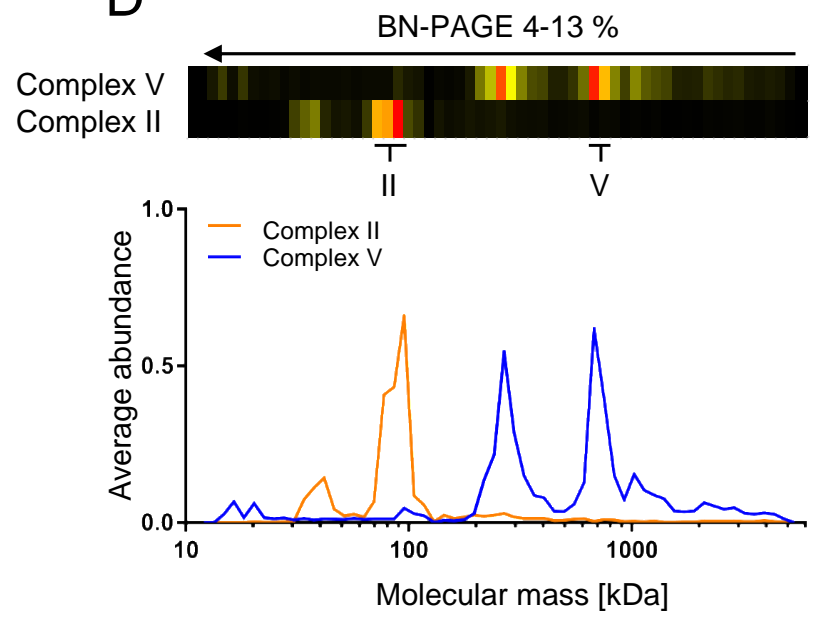

E

\section{PLN KO}

BN-PAGE 4-13\%
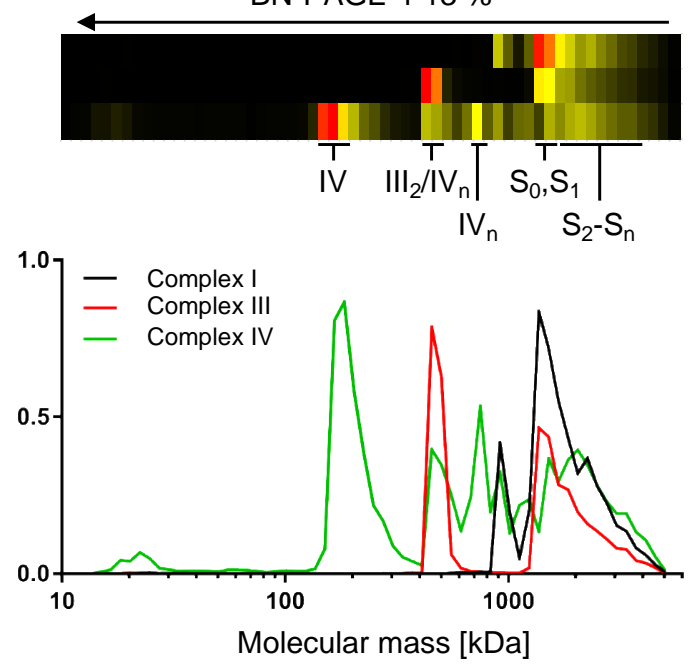

F

BN-PAGE 4-13\%
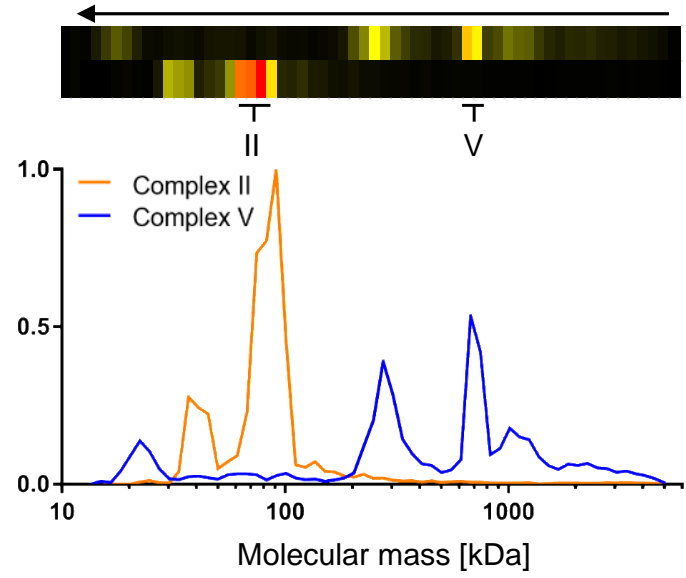

relative abundance

\section{Figure 11 Complexome profiling of OXPHOS complexes}

A+B, Heat maps of OXPHOS complexes in WT and PLN KO VCM were used as methodical quality control and for mass calibration. Respiratory chain complexes are indicated as complex I (I), complex II (II), complex V (V), complex III (III $\mathrm{L}_{2}$, dimer), domplex IV (IV, IV $\mathrm{n}_{\mathrm{n}}$, multimer), and supercomplexes (S$\left.\mathrm{S}_{n}\right)^{74}$. C-F, Average abundance of all OXPHOS subunits presented in heat maps and migration profiles in WT and PLN KO VCM. Modified from Alsnia et al. ${ }^{61}$

Data clustering revealed the $\mathrm{Na}, \mathrm{K}-\mathrm{ATPase} \alpha_{1}-\beta_{1}$ complex $^{90}$ together with its regulator phospholemman (PLM) (Figure 12A) ${ }^{91}$. Remarkably, different states of the Na,K-ATPase complex were identified through a mass shift, namely the active complex $\left(\alpha_{1}-\beta_{1}\right)$ or inhibited 
by PLM binding ( $\alpha_{1}-\beta_{1} /$ PLM) (Figure $12 A$, inset). This example shows the sensitivity and resolution of this method to distinguish between molecular complexes with different functional states. Furthermore, PLN and SERCA2a showed a similar migration pattern and are part of macromolecular complexes with increasing abundance in the high molecular range (Figure 12B). Even PLN monomers and pentamers were captured. Interestingly, SERCA2a complexes were disrupted in PLN KO cardiomyocytes ${ }^{61}$.

A
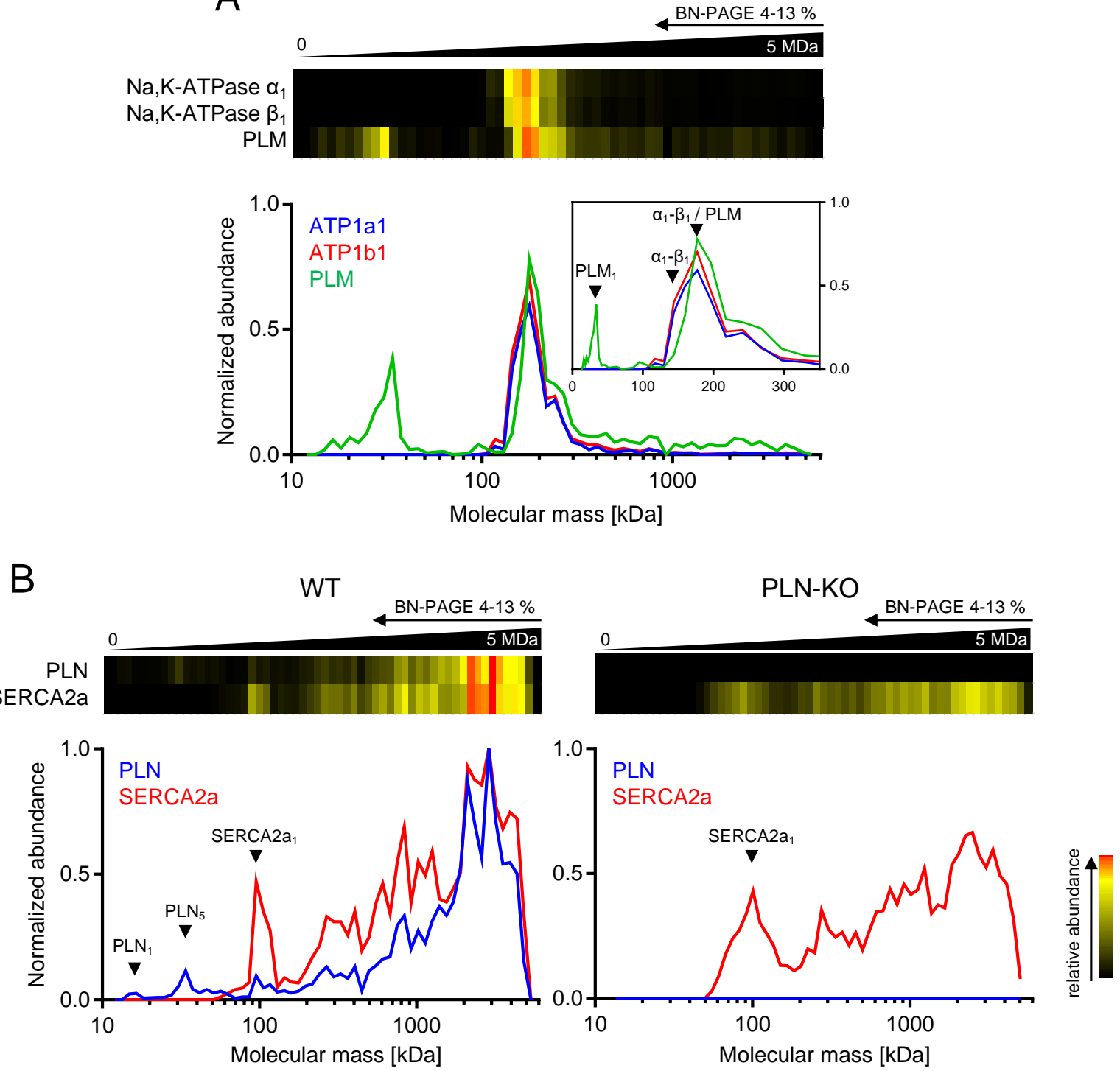

Figure 12 Complexome profiling of mouse ventricular cardiomyocyte membranes

Heat maps representing the complexome profiling data of mouse ventricular cardiomyocytes (VCM). Colors indicate the intensity gradient (look-up-table) by its relative abundance. The same data were plotted as line profiles showing the molecular weight on a logarithmic scale versus normalized protein abundance. A, In WT VCM, Na,K-ATPase subunits $\alpha 1$ and $\beta 1$ showed the same migration pattern, whereas its regulator phospholemman (PLM) showed two main peaks. The inset shows the mass range 
from 0 to $350 \mathrm{kDa}$ were a mass shift of the Na,K-ATPase complex $(\alpha 1-\beta 1)$ due to the binding of PLM can be recognized ( $\alpha 1-\beta 1$ / PLM). B, WT and PLN KO complexome profiling data for PLN and SERCA2a. Mono- and pentameric PLN and monomeric SERCA2a (arrowheads) were detected at the expected molecular mass and showed a similar migration pattern at the high molecular range. In PLN KO, SERCA2a distribution was altered with a reduced signal. Figures were modified from Alsina et al. ${ }^{61}$.

Further analysis showed that protein phosphatases and kinases could be identified by complexome profiling (Figure 13). The catalytic subunits $\alpha$ and $\beta$ of the protein phosphatase 1 (PP1) are abundant in the same gel slice, whereas the regulatory subunit PPP1R3A was found in the high molecular weight range. It is interesting to note that this complex was disrupted in PLN KO cardiomyocytes ${ }^{61}$. Also, the protein kinase A, which phosphorylates PLN $^{92}$ was detected in an inactive tetramer composed of two catalytic $C$ - $\alpha$ subunits and the two regulatory $\mathrm{RI}-\alpha$ and RII- $\alpha$ subunits (Figure 13B,E) ${ }^{93,94}$. Upon cAMP binding ${ }^{93}$, the catalytic subunits are released and active, reflecting the indicated peak at 30-40 kDa (Figure 13B,E). The second kinase which phosphorylates PLN is the calcium/calmodulin-dependent protein kinase 2 (CaMK2). In addition to the predominant cardiac-specific isoform CaMK2 $\delta^{95}$, CaMK2 $\alpha$ was detected. Both isoforms were abundant in macromolecular complexes, similar to PPP1R3A ${ }^{61}$. 
A
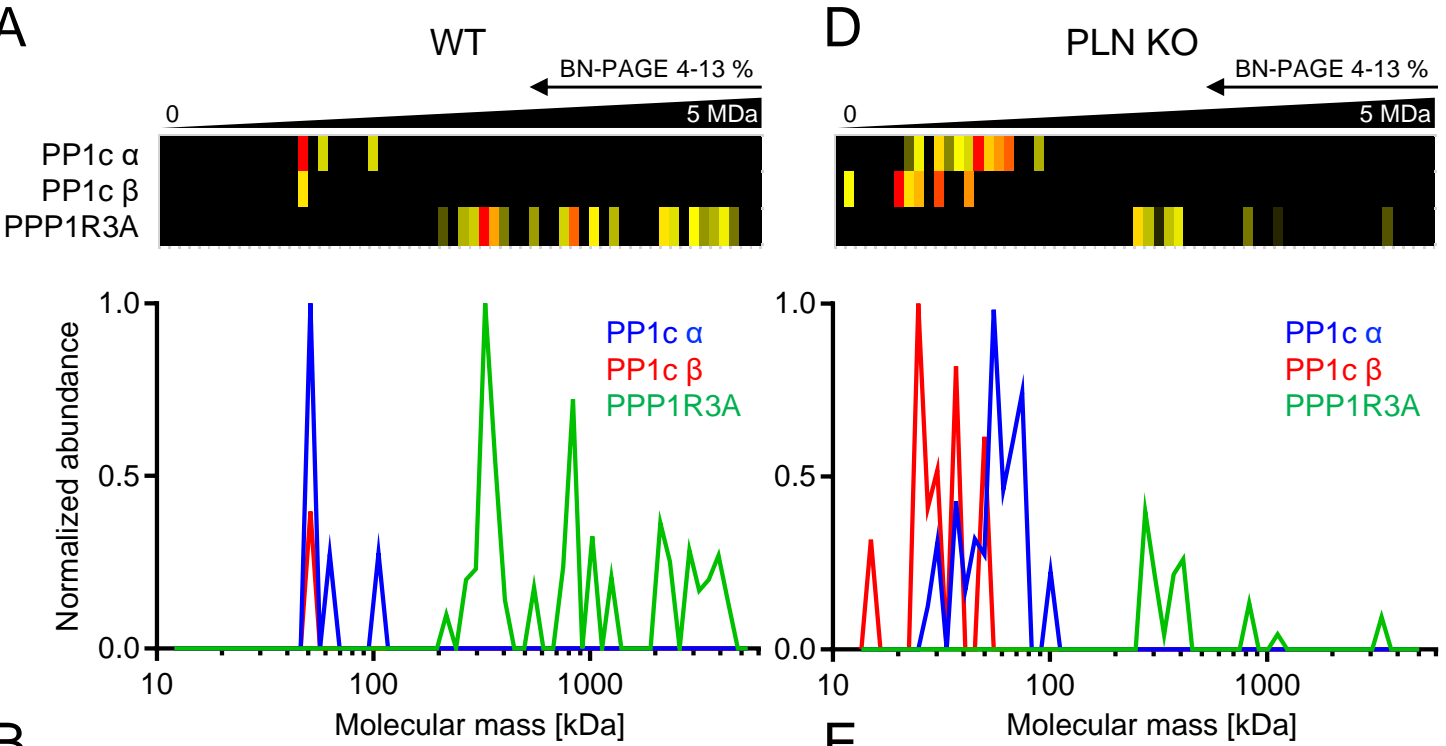

$B$

Molecular mass $[\mathrm{kDa}]$

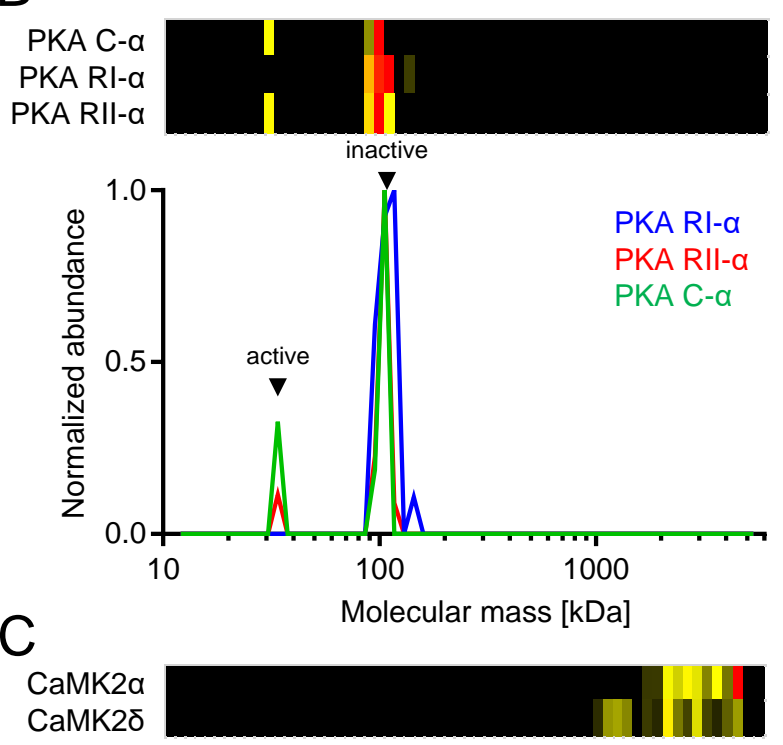

E
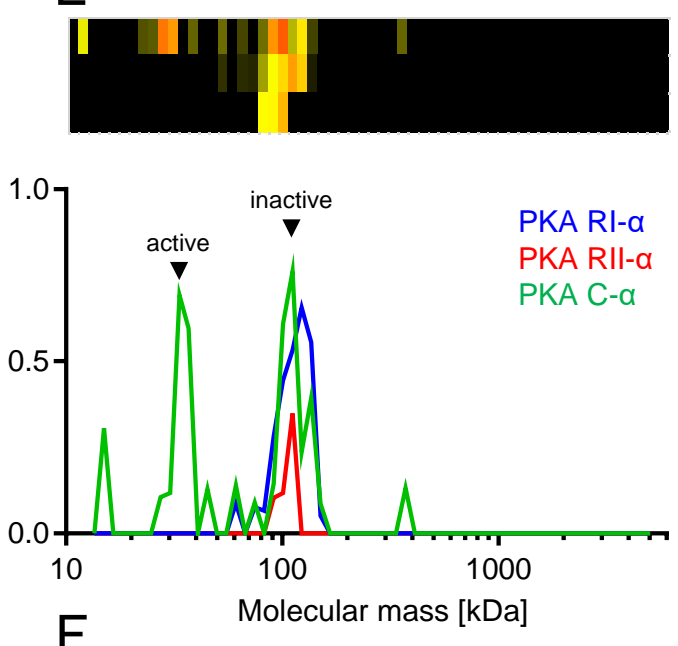

$F$
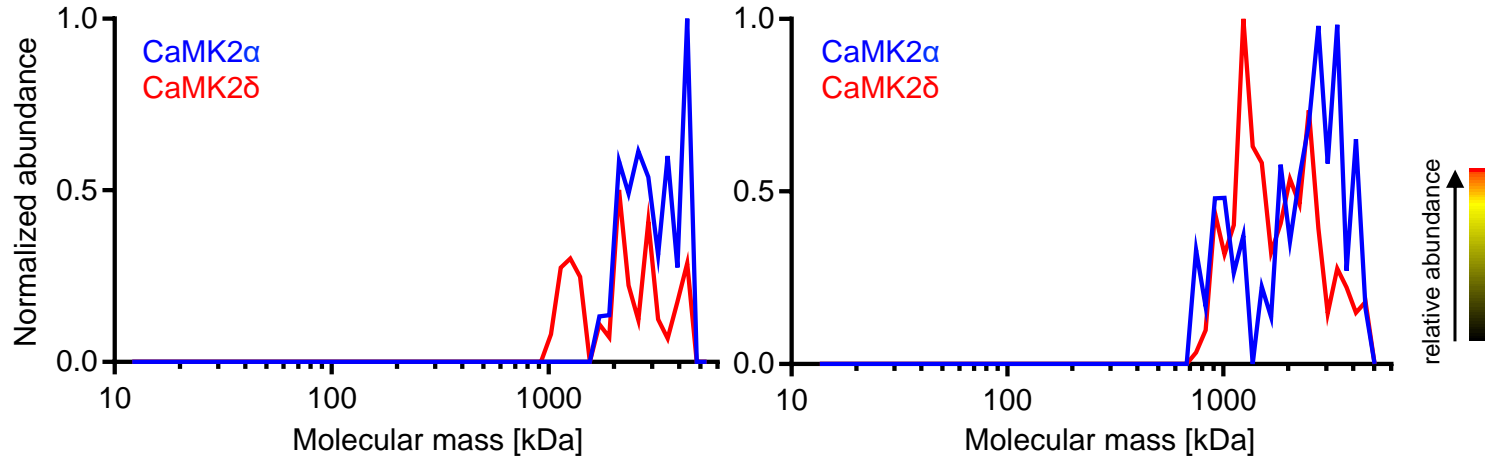

Figure 13 Complexome profiling of kinase and phosphatase distributions

WT and PLN KO complexome profiling data of mouse ventricular cardiomyocytes (VCM). A+D, Protein phosphatase 1 (PP1) catalytic subunits $\alpha$ and $\beta$ show a different migration pattern compared to the regulatory subunit 3A (PPP1R3A). Ablation in PLN resulted in reduced abundance and altered distribution of PPP1R3A in high molecular weight complexes. The figure was modified from Alsina et al. (Fig. 6) ${ }^{61}$. B+E, Protein kinase A (PKA) catalytic subunit alpha (C- $\alpha$ ) migrated as a monomer at $30-40$ 
$\mathrm{kDa}$ (active) and bound as a dimer to the regulatory subunits RI- $\alpha$ and RII- $\alpha$ in a complex at $>100 \mathrm{kDa}$ (inactive). In PLN KO, C- $\alpha$ distribution is altered (E). C+F, Calcium/calmodulin-dependent protein kinase 2 (CaMK2) subunits $\alpha$ and $\delta$ were found in high molecular weight complexes $>1000 \mathrm{kDa}$ and show increased signals in PLN KO.

Additionally, complexome profiling revealed PPP1R3A together with RyR2 and the PLN/SERCA2a in a complex suggested by overlapping migration profiles (Figure 14). Interestingly, these high molecular weight complexes are disrupted in the absence of PLN. This finding is part of the publication by Alsina et al. ('Loss of Protein Phosphatase 1 Regulatory Subunit PPP1R3A Promotes Atrial Fibrillation' Circulation 2019) ${ }^{61}$.

In general, these data show that complexome profiling is a promising application for the analysis of cardiac macromolecular protein complexes. A conceptually new protein complex that includes PLN, SERCA2 and RyR2 have been identified, including PPP1R3A as coregulatory mechanism for $\mathrm{PP} 1$ targeting ${ }^{61}$.

Importantly, hierarchical cluster analysis of the migration profiles revealed sarcolemmal membrane-associated protein (SLMAP) as a potential new interacting protein of PLN, comigrating with the PLN/SERCA2a complex. 

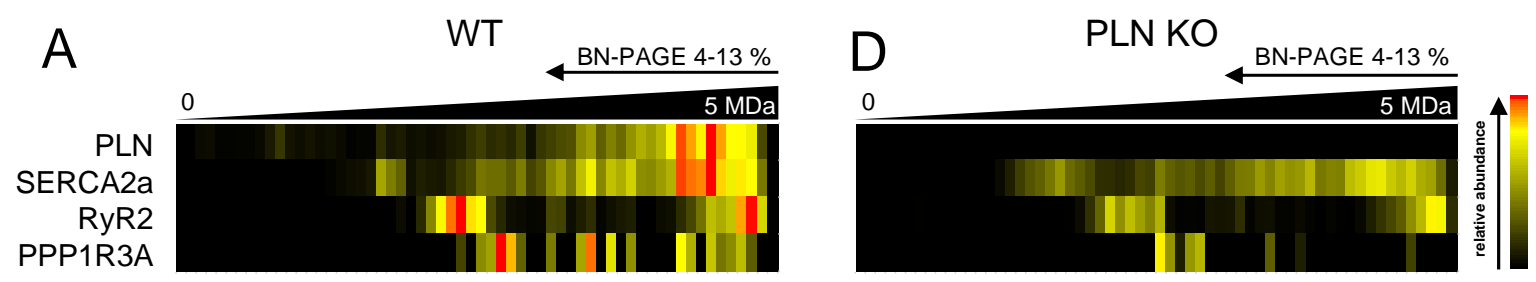

B
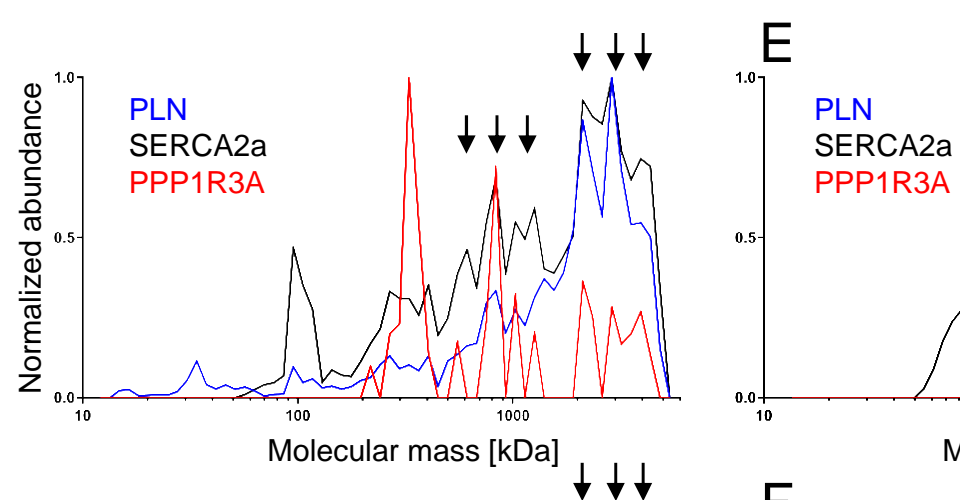

SERCA2a
PPP1R3A

C
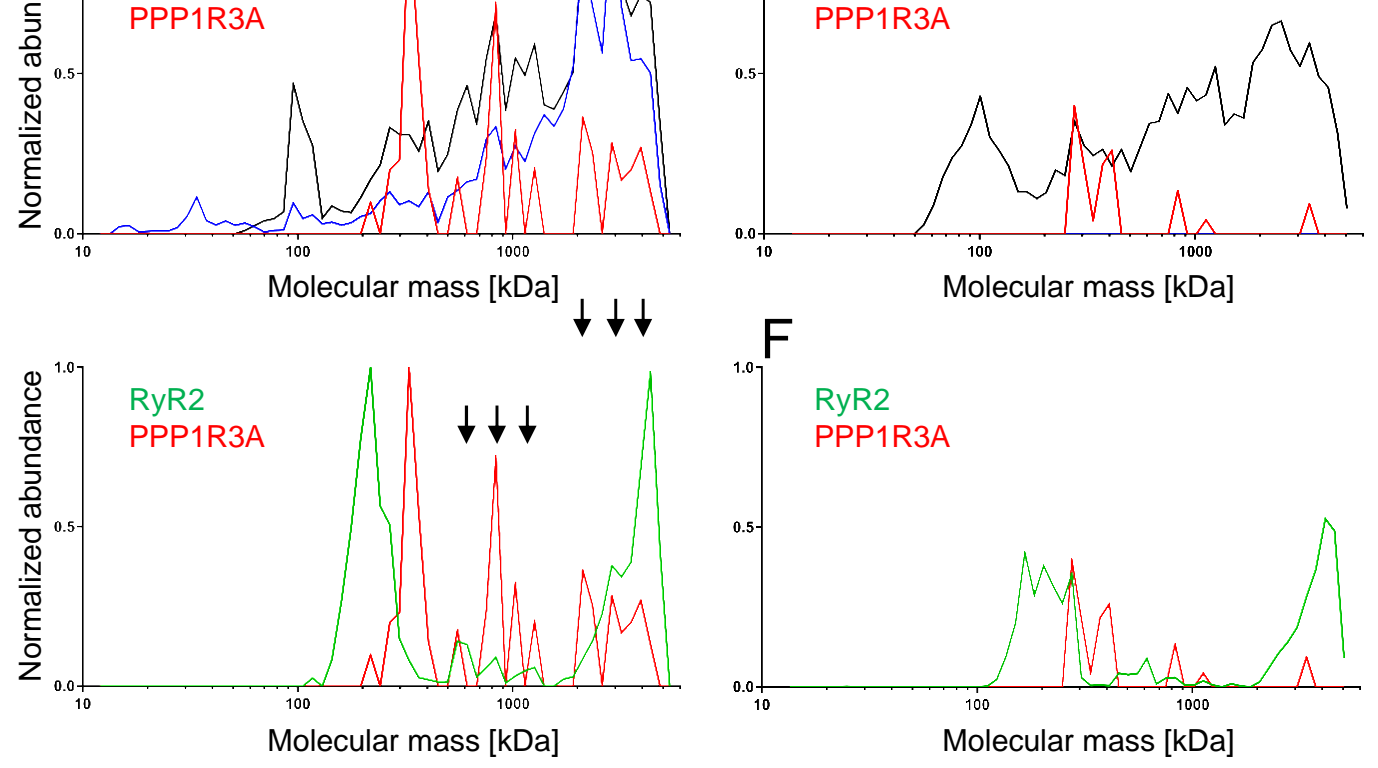

Figure 14 Complexome profiling shows novel ' $\mathrm{SR} \mathrm{Ca}^{2+}$ cycling complex'

WT and PLN KO complexome profiling data of mouse VCM. A+D, Heat maps representing the complexome profiling data for PLN, SERCA2a, RyR2, and PPP1R3a. Colors indicate the intensity gradient of normalized abundance (look-up-table). $\mathbf{B}+\mathbf{E}$, The same data were plotted as line profile for PLN, SERCA2a and PPP1R3A showing the molecular weight versus normalized protein abundance. Arrows indicate the overlap of abundance profiles suggesting a macromolecular complex, also together with RyR2 $(\mathbf{C}+\mathbf{F})$, whereas PLN KO leads to the reduced abundances and the loss of high molecular weight complexes $>800 \mathrm{kDa}$. Modified from Alsnia et al. ${ }^{61}$

\subsubsection{SLMAP forms high molecular weight complexes}

The analysis of native membrane complexes by blue native gel electrophoresis is a state-ofthe-art method to analyze known protein-protein interactions, track changes but also to characterize novel protein complexes. As described in the previous chapter, PPP1R3A was shown to bind to the PLN/SERCA2a complex and the ryanodine receptor 2 channel macromolecular protein assemblies by complexome profiling in line with additional experimental data ${ }^{61}$.

Additionally, hierarchical cluster analysis from mouse ventricular cardiomyocytes revealed that the sarcolemmal membrane-associated protein (SLMAP) comigrates with the PLN/SERCA2a 
complex (Figure 15A). Upon PLN ablation, the SLMAP signals were increased and showed higher similarity to the SERCA2a profile compared to wildtype (Figure 15B). At the same time, SERCA2a distribution was altered and decreased as described before ${ }^{61}$. SLMAP is a tailanchored transmembrane protein ${ }^{78}$, confirming the SLMAP enrichment in membrane fractions of WT and PLN KO ventricular cardiomyocytes (Figure 15C). SLMAP can give rise to several isoforms as a result of alternative splicing ${ }^{78,79}$. In mass spectrometry, only peptides shared by all isoforms are detected the coiled-coil domains 3 and 4 particularly (Figure 7). Because LCMS/MS cannot discriminate between the isoforms, we quantified the abundance of different SLMAP isoforms in high molecular weight complexes by 2D BNE/SDS-PAGE experiments (Figure 15D). Immunoblotting showed SLMAP isoforms in a heteromeric complex at $\sim 200 \mathrm{kDa}$ in the high molecular range (>200 kDa). Taken together, all SLMAP isoforms are abundant in high molecular weight complexes and show a similar migration profile within the BN gel, suggesting a shared macromolecular complex. 

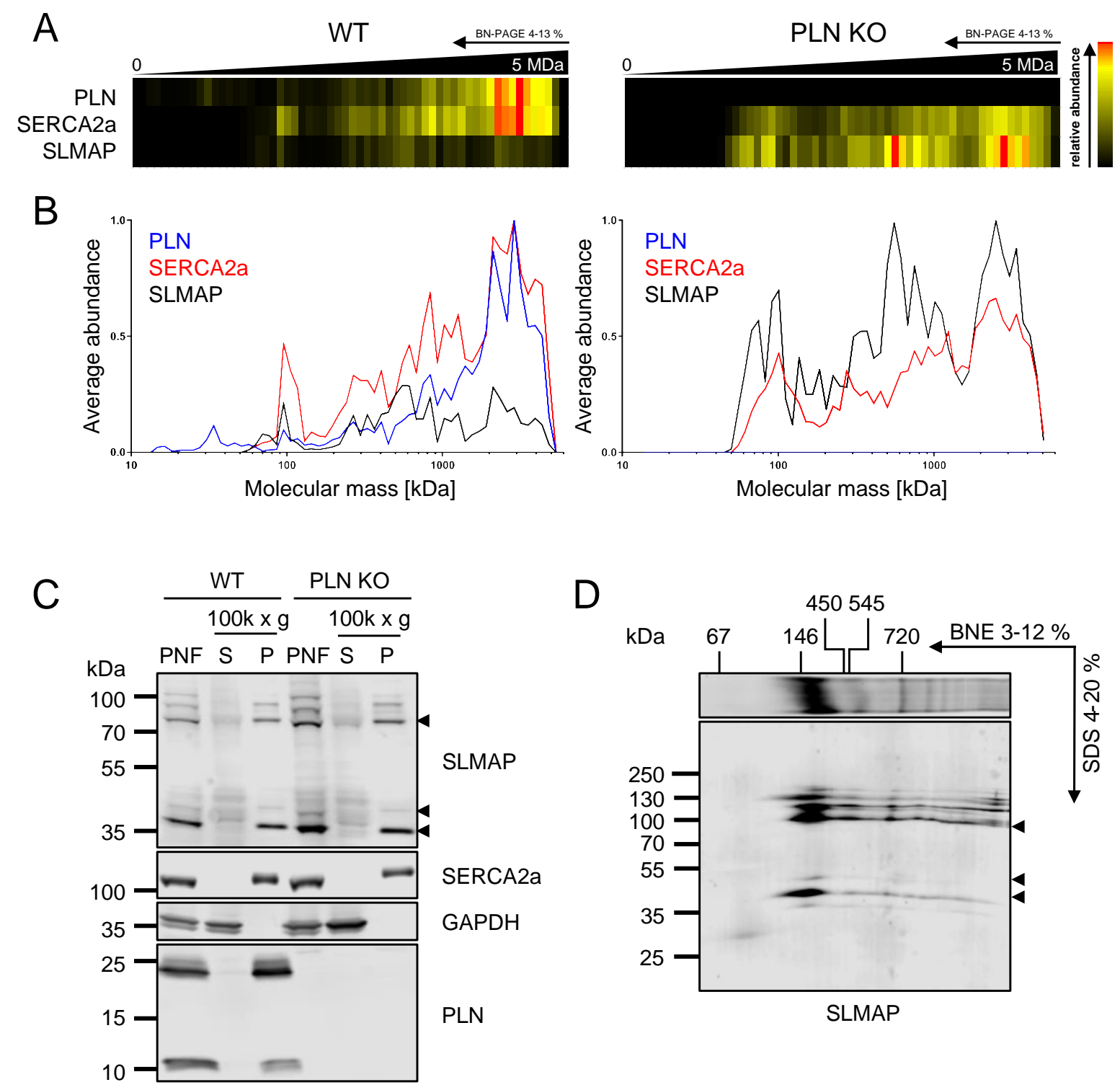

\section{Figure 15 SLMAP isoforms form high molecular weight complexes}

A, Heat maps representing the complexome profiling data for PLN, SERCA2a, and SLMAP of mouse WT and PLN KO ventricular cardiomyocytes (VCM). Colors indicate the intensity gradient of normalized abundance (look-up-table). Membranes were digitonin-solubilized, separated on a 4-13\% blue native (BN) gel and subjected to mass spectrometry (LC-MS/MS). Data were maximum normalized over WT and PLN KO signals. B, The same data were plotted as line profile showing the molecular weight versus normalized protein abundance. In mouse WT VCM, SLMAP showed a similar migration pattern with the PLN/SERCA2a complex. Ablation in PLN KO resulted in an increase of the SLMAP signals compared to WT, whereas the SERCA2a distribution was altered with a reduced signal. C, Detergent-free perinuclear fractions (PNF) of mouse WT and PLN KO ventricular cardiomyocytes were ultracentrifuged at $100.000 \times \mathrm{g}$ and samples separated on a $4-20 \%$ SDS gel. Immunoblot showing enrichment of SLMAP, SERCA2a, and PLN in the membrane fractions (P). GAPDH was used as a cytosolic marker protein enriched in the supernatant (S). Arrowheads indicate the different SLMAP isoforms. D, Immunoblotting of a blue native gradient gel (BNE) was used to identify high molecular weight complexes of SLMAP 
isoforms in mouse WT VCM. For this, enriched membranes were digitonin solubilized, separated in the first dimension by BNE, and in the second dimension by SDS gradient gel under denaturing conditions. SLMAP isoforms form a heteromeric complex at $\sim 200 \mathrm{kDa}$ and are present in higher molecular weight complexes $(>200 \mathrm{kDa})$.

\subsubsection{SLMAP localization and PLN proximity in mouse ventricular cardiomyocytes}

As the localization of SLMAP depends on its transmembrane domain and has been localized to different subcellular compartments ${ }^{81,96}$, we immunolabeled SLMAP for confocal and STED co-immunofluorescence imaging with Caveolin 3 (CAV3) and PLN, respectively. Whereas confocal microscopy showed a transversal striated pattern for SLMAP, STED nanoscopy resolved clearly separated SLAMP and CAV3 signals, i.e. with no signal overlap, in WT and PLN KO mouse ventricular cardiomyocytes (Figure 16A). In contrast, SLMAP signals are immediately adjacent and surrounded by PLN signals indicating localization in the sarcoplasmic reticulum membrane (Figure 16B). 


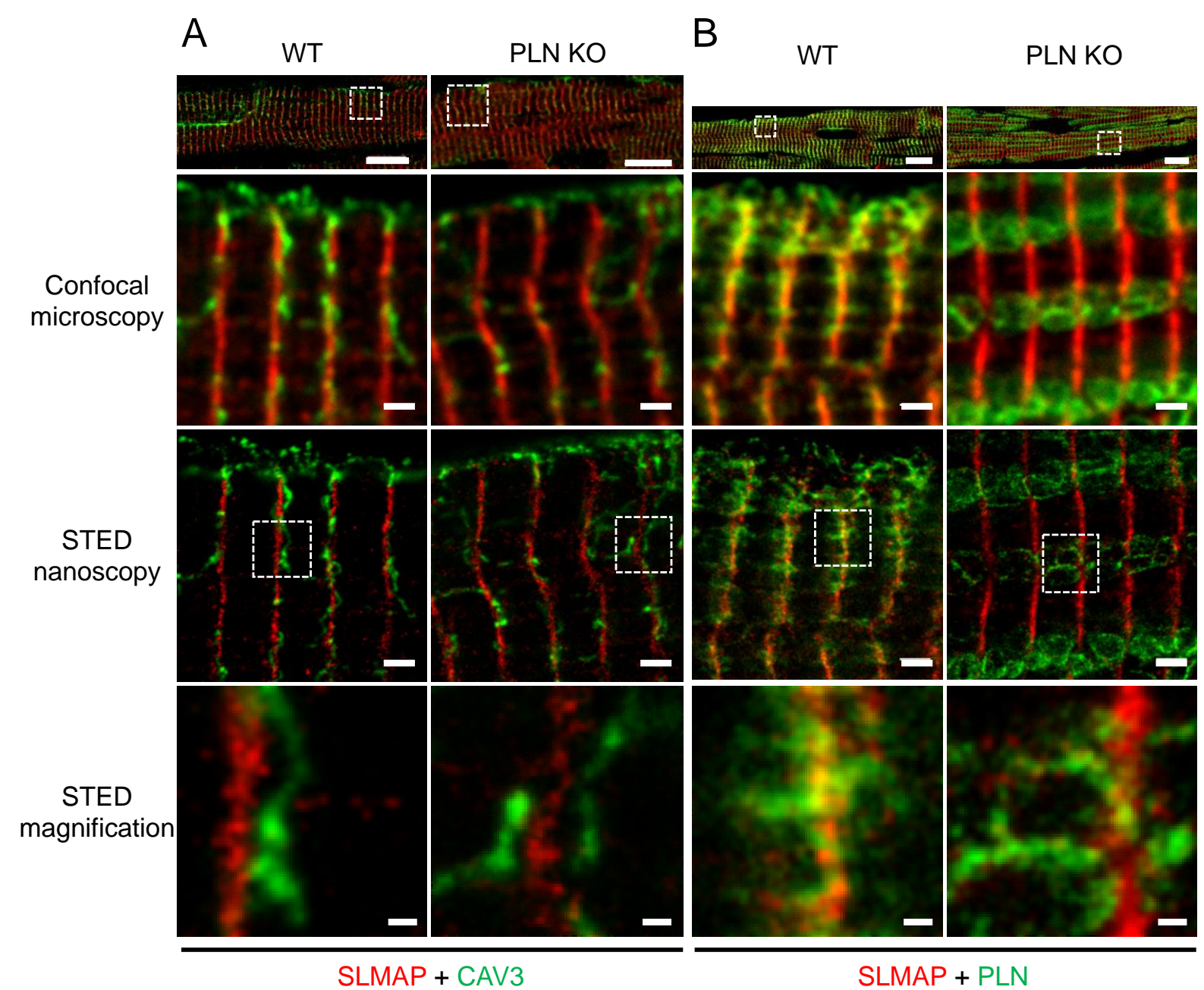

Figure 16 SLMAP shows a transversal striated pattern in mouse ventricular tissue

Confocal and STED co-immunofluorescence imaging was used for mouse WT and PLN KO ventricular tissue from paraffin-embedded histological sections stained with SLMAP and CAV3 or PLN, respectively. Dashed boxes indicate magnified regions where STED superresolution nanoscopy resolved a separated localization of SLMAP and CAV3 (A). In contrast, the SLMAP signals were immediately adjacent to and surrounded in subcellular domains by PLN (B). In PLN KO, the PLN antibody showed signals not present in WT, which were related to unspecific mitochondrial background. Scale bars: top $10 \mu \mathrm{m}$; Confocal microscopy $1 \mu \mathrm{m}$; STED nanoscopy $1 \mu \mathrm{m}$; STED magnification $200 \mathrm{~nm}$.

To explore the hypothesis that SLMAP and PLN can directly interact, we performed coimmunoprecipitation experiments (Figure 17). Interestingly, all three SLMAP isoforms were successfully co-purified with PLN. In summary, these data confirm close proximity of PLN and SLMAP, suggesting a functional complex at the sarcoplasmic reticulum. 


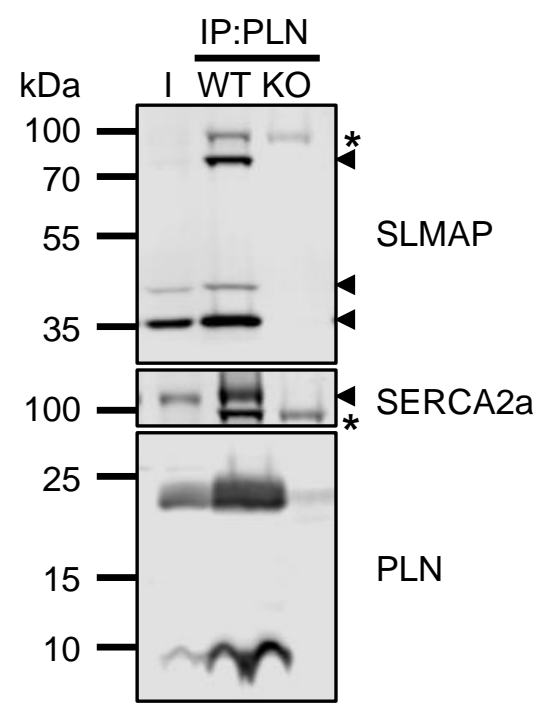

Figure 17 SLMAP is co-purified with PLN by affinity purification

PLN-targeted co-immuno purification (IP) of mouse ventricular tissue (WT), followed by immunoblotting, confirmed the protein-protein interaction between PLN and SLMAP. PLN KO ventricular tissue (KO) was used as a negative control. The previously established PLN-SERCA2a interaction was used as a positive control. Arrowheads indicate the different SLMAP isoforms and SERCA2a. Asterisks indicate IgG heavy chain detected by the secondary antibody. I: Input; $n=3$.

\subsubsection{SLMAP expression is altered in PLN KO mouse hearts and cardiac hypertrophy}

To further explore the SLMAP abundance change observed in PLN KO (Figure 15), we investigated the SLMAP protein expression in ventricular heart tissue by immunoblotting. Quantifications confirmed the complexome profiling data by showing a significant increase in SLMAP1 and SLMAP3 expression in PLN KO ventricles (Figure 18A,B), whereas SERCA2a expression was reduced (Figure 18A,C). RyR2 was not found to change significantly on the protein level. 
A

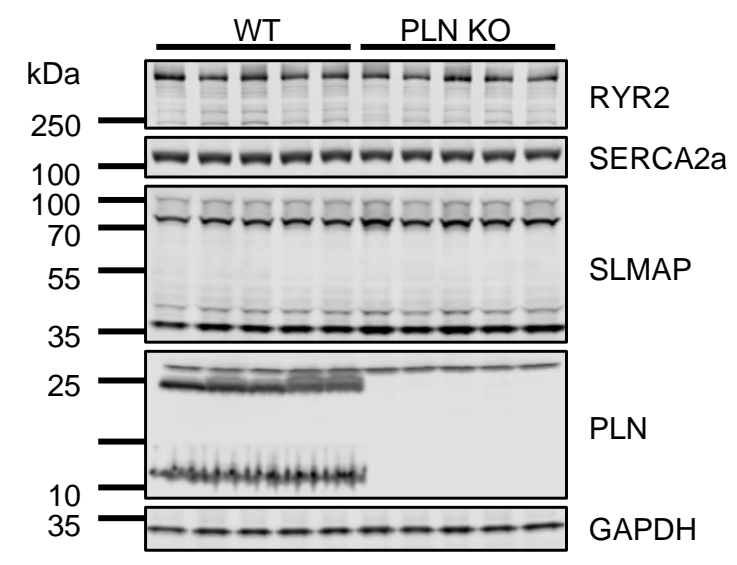

B

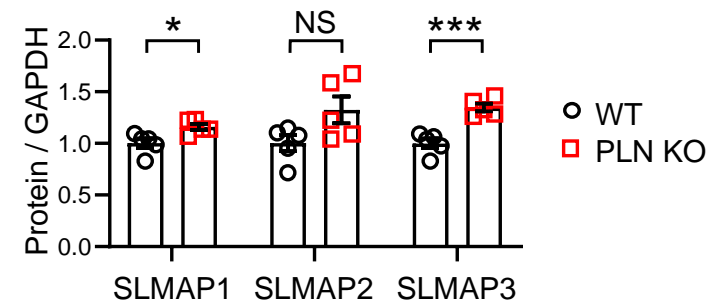

C

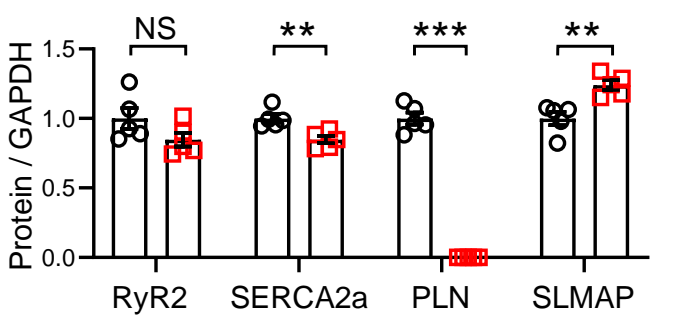

Figure 18 Mouse ventricular tissue expression of SLMAP in WT and PLN KO mouse hearts

A, Immunoblotting of ventricular tissue lysates from PLN wildtype (WT) versus knockout (PLN KO) of adult mice. B, Quantifications of immunoblots normalized to GAPDH show a significant increase of SLMAP1 and SLMAP3 expression and total SLMAP (C) in PLN ablated tissue. In contrast, SERCA2a expression is significantly decreased $(C)$. A representative GAPDH immunoblot is shown, while each blot was normalized to its GAPDH signal. Data represent means and $\pm S E M, n=5$. ${ }^{*} P<0.05$; ${ }^{* *} P<0.01$; ${ }^{* * *} P<0.001$; NS: not significant, Student's t-test.

Not only PLN KO hearts but also hearts during the development of hypertrophy and heart failure underwent molecular changes ${ }^{97,55,98}$. It has been reported that SLMAP3 protein expression is increased in hypertrophic mouse hearts ${ }^{98}$. We hypothesized that all SLMAP isoform expression levels change during heart failure. To test this, we used a model of transaortic constriction (TAC) to study SLMAP expression changes during induced left ventricular hypertrophy in mice.

Immunoblot experiments of WT mice revealed a significant increase of all SLMAP isoform protein expression four weeks after TAC (Figure 19A,B), whereas RyR2 and SERCA2a were significantly decreased (Figure 19A,C). PLN was not changed. Study design and sample preparation were carried out by Dr. Sören Brandenburg (Dept of Cardiology and Pneumology, University Medical Center Göttingen), and tissue lysates were kindly provided for this work. 
A

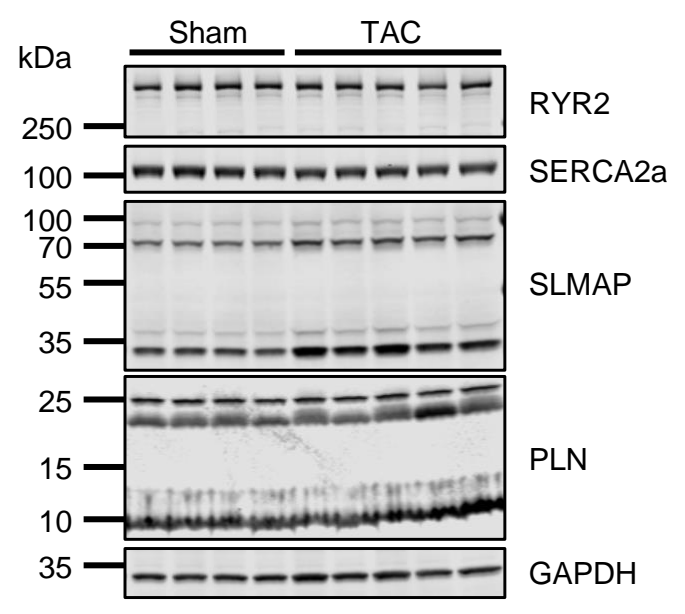

B
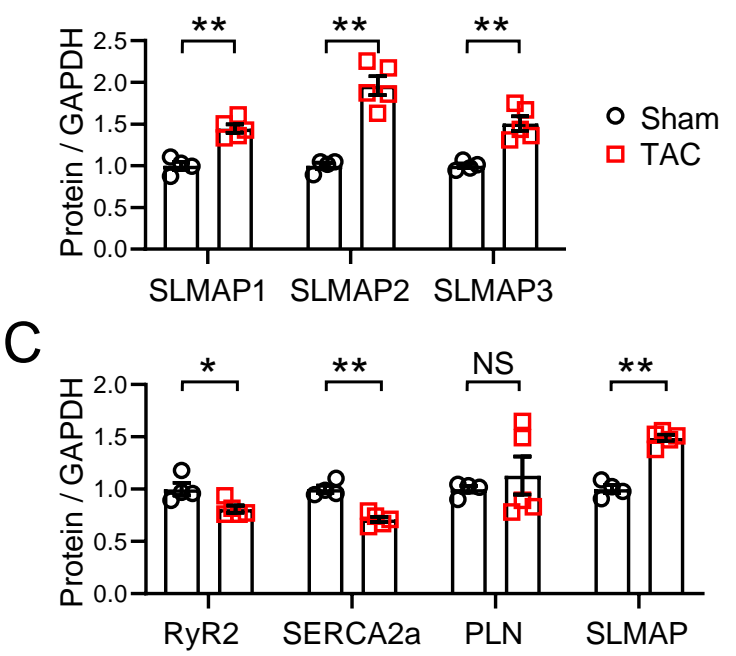

Figure 19 SLMAP protein expression is increased in mouse ventricular tissue after TAC

A, Immunoblots of ventricular tissue lysates of WT mice 4 weeks after TAC intervention. B, Quantification of immunoblots normalized to GAPDH shows a significantly increased expression of all SLMAP isoforms. In contrast, RyR2 and SERCA2a were significantly reduced after TAC. PLN expression was not changed significantly (C). A representative GAPDH immunoblot is shown, while each blot was normalized to its GAPDH signal. Data represent means and $\pm S E M, n=4$ minimum. ${ }^{*} \mathrm{P}<0.05 ;{ }^{* *} \mathrm{P}<0.01 ;{ }^{* * *} \mathrm{P}<0.001$; NS: not significant, Student's t-test.

To investigate how SLMAP protein expression changes in TAC operated PLN KO hearts, we performed these experiments in collaboration with the service unit of the SFB1002 (University Medical Center Göttingen). It has been shown that PLN KO mice can compensate against a sustained TAC intervention similar to WT mice ${ }^{97}$. The TAC operated PLN KO hearts showed a significant increase for SLMAP2 (Figure 20A,B), whereas total SLMAP was un-changed compared to WT sham (Figure 20C). SERCA2a expression was reduced in PLN KO, similar to WT hearts. RyR2 protein expression was not changed. Taken together, these data confirm the increased expression of SLMAP in PLN KO and TAC hearts by RNA microarray and immunoblotting. Importantly of note that the group size $n=2$ of PLN KO after TAC intervention was not sufficient for statistics. 
A

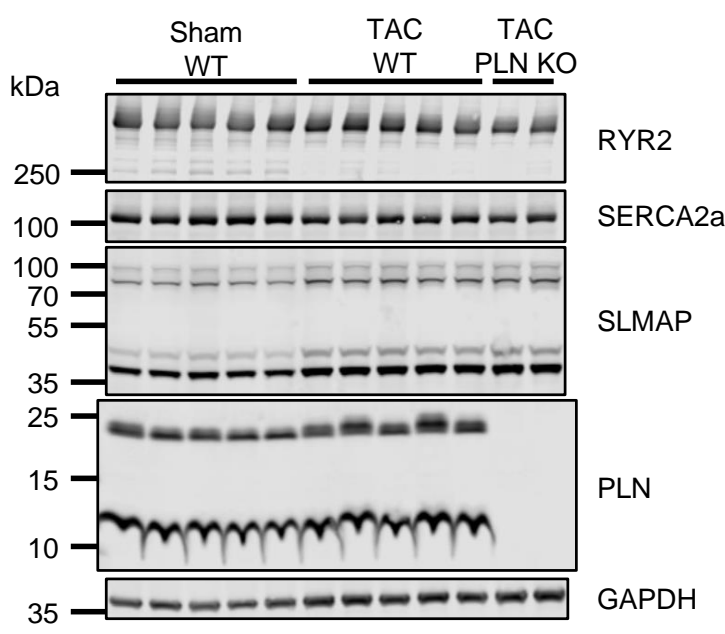

B

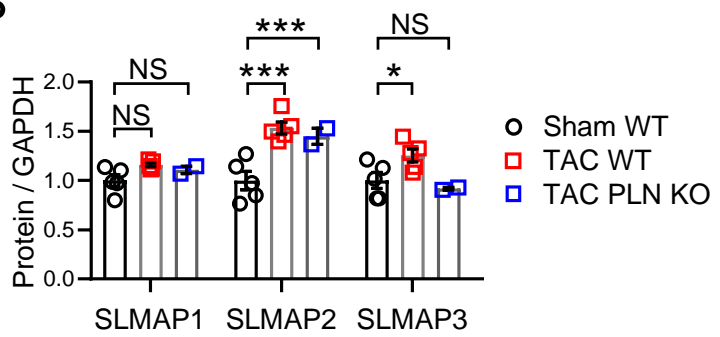

C

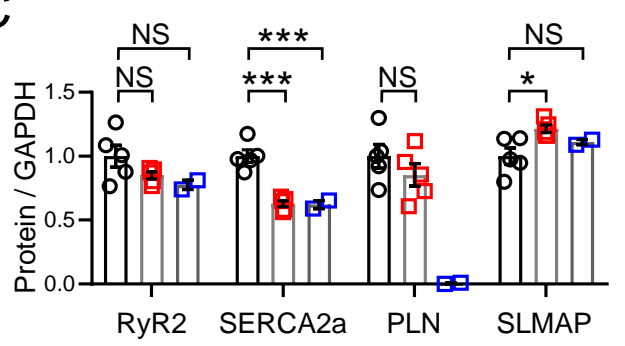

Figure 20 SLMAP protein expression is unchanged in PLN KO ventricular tissue after TAC

A, Immunoblots of ventricular tissue lysates show sham versus TAC 4 weeks after the intervention of WT and PLN KO mice. B, Quantification of immunoblots normalized to GAPDH show significantly increased expression of SLMAP2 and SLMAP3 in WT after TAC. In PLN KO, only SLMAP2 was significantly increased, whereas SLMAP1 and SLMAP3 show no changes compared to sham WT. C, SERCA2a expression was reduced after TAC in WT and PLN KO, whereas RyR2 and PLN expression were not changed significantly. Total SLMAP expression was only significantly increased in WT hearts after TAC. A representative GAPDH immunoblot is shown, while each individual blot was normalized to its GAPDH signal. Data represent means and \pm SEM, $n($ Sham WT $)=5, n(T A C$ WT $)=5, n(T A C$ PLN $K O)=2$, gender mixed mice. ${ }^{*} P<0.05$; ${ }^{* *} P<0.01$; ${ }^{* * *} P<0.001$; NS: not significant, Student's t-test.

\subsubsection{SLMAP in human iPSC-derived cardiomyocytes}

To investigate the expression and localization of SLMAP in iPSC-derived cardiomyocytes we performed immunoblots, confocal and STED superresolution microscopy (Figure 21). Immunoblots showed robust and increasing expression of SLMAP isoforms after 60 and 90 days of differentiation in iPSC-derived cardiomyocytes (iPSC-CM) (Figure 21A). Coimmunofluorescence staining revealed SLMAP signals, adjacent and surrounded by PLN and SERCA2a signals, in a clustered organization (Figure 21B). 

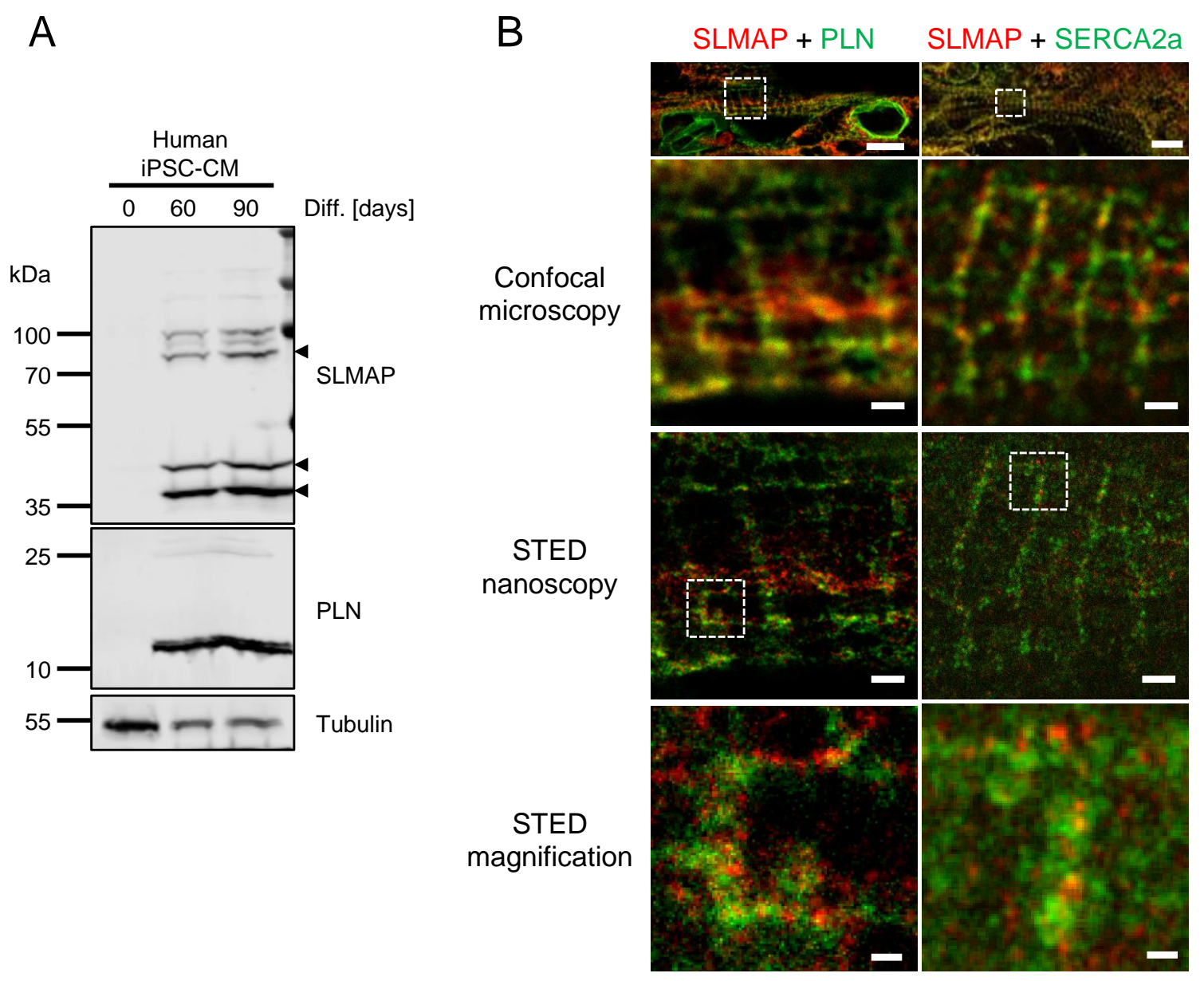

Figure 21 SLMAP is expressed in human iPSC-derived cardiomyocytes

A, Immunoblotting of human iPSC-derived cardiomyocytes (iPSC-CM) after indicated days of differentiation. SLMAP and PLN are robustly expressed upon differentiation. Tubulin was used as an expression marker. Arrowheads indicate SLMAP isoforms. B, Confocal and STED coimmunofluorescence imaging of SLMAP with PLN or SERCA2a in 60 days differentiated human iPSCCM. STED magnifications show clustered signals for SLMAP and overlap partially with PLN or SERCA2a signals. Scale bars: top $10 \mu \mathrm{m}$; Confocal microscopy $1 \mu \mathrm{m}$; STED nanoscopy $1 \mu \mathrm{m}$; STED magnification $200 \mathrm{~nm}$.

Differentiation and cultivation of human iPSC were carried out by Dr. Lukas Cyganek and the Stem Cell Unit (University Medical Center Göttingen).

In summary, SLMAP is sufficiently expressed in human iPSC-CM and will be targeted by CRISPR/Cas9 to generate an SLMAP knockout and study the potentially functional complex of PLN and SLMAP. 


\subsection{Discussion}

The exploration of novel PLN interaction partners is of high importance to understand how it is molecularly regulated and to develop rationales for new therapeutic interventions for patients and animals with heart disease.

This study was designed to identify potential new protein interactions of phospholamban and to explore native protein complexes in cardiac membranes. A recently developed complexome profiling approach ${ }^{72,74,73}$ was applied to study native protein complexes involved in muscle contraction in cardiac membranes from isolated mouse ventricular cardiomyocytes.

Cardiac membranes were isolated under native conditions and separated by blue native gel electrophoresis ${ }^{58}$, followed by mass spectrometry analysis ${ }^{59}$. Abundance profiles showed the migration of each detected protein in the gel and revealed several macromolecular complexes. For quality control, the mitochondrial OXPHOS complexes were analyzed as to their assembly into so-called supercomplexes is well described ${ }^{74,89}$.

As a result, PLN and SERCA2a were found to be abundant in macromolecular complexes, whereas these complexes were altered with reduced abundance upon PLN ablation. Furthermore, a conceptually new macromolecular complex comprised of RyR2 and the PLN/SERCA2a complex has been identified ${ }^{61}$. This complex was accompanied by the regulatory subunit PPP1R3a targeting the protein phosphatase 1 to PLN $^{99}$ and the RyR2 protomers of the tetrameric channel ${ }^{61}$.

Additionally, a potentially new interacting partner of PLN was identified by migration profile clustering, the sarcolemmal membrane-associated protein (SLMAP). Together with STED superresolution microscopy and co-immunoprecipitation experiments, an association of SLMAP and PLN is suggested. Furthermore, in PLN KO heart tissue, SLMAP expression was found to be increased, as well as in a cardiac hypertrophy mouse model after 4 weeks of transaortic constriction. Taken together, a potential role during hypertrophic remodeling and heart failure development was identified for SLMAP. In order to analyze a potential role of SLMAP in intracellular $\mathrm{Ca}^{2+}$-cycling, B-adrenergic stimulation, and contraction, a CRISPR/Cas9 targeted knockout in human induced pluripotent stem cell-derived cardiomyocytes was designed for future analysis.

\subsubsection{Isolation and solubilization of cardiac membranes for BN-PAGE}

The recently developed bottom-up proteomics approach complexome profiling ${ }^{72,74,73}$ was applied to identify PLN protein interactions in cardiac membranes. In order to enrich cardiac SR membranes from isolated ventricular cardiomyocytes, differential centrifugation ${ }^{87}$ was used to separate different cellular compartments and membrane organelles. Sample preparation is 
critical for protein complex stability, retrieval, and gel separation quality ${ }^{100}$. To maintain stable protein complexes, a detergent-free homogenization buffer was used. However, under these conditions, a sufficient SR separation from other cell compartments was not possible. This is not unexpected, as mitochondria are highly abundant in cardiomyocytes ${ }^{101}$, and physical SR/ER-mitochondria contacts known as mitochondria-associated ER membranes (MAM), with physiological roles, are present ${ }^{102}$. Finally, total cardiac membranes were used for complexome profiling, taken into account that the complexity of the sample may lead to more missing peak volume values and reduced dynamic range ${ }^{100}$. As this method was established for mitochondrial complex analysis ${ }^{73,74}$, the respiratory complexes were used as internal standard and quality control.

Furthermore, the choice of detergent has a substantial impact on complex solubilization efficiency, membrane complex representation, and stability ${ }^{103}$. DDM and digitonin are nonionic, relatively mild detergents that may maintain the native association between several proteins and are, thus, widely used for blue native separation and mass spectrometry ${ }^{72,74,73,104,105}$. For each protein of interest and biological starting material, the preservation of protein interactions may vary between detergents. Therefore, cardiac membrane solubilization with digitonin was used, confirming PLN and SERCA2a abundance in high molecular weight complexes.

In order to separate protein complexes from biological membranes, blue native gel electrophoresis was used, reproducing a mass range from $10 \mathrm{kDa}$ to $10 \mathrm{MDa}^{58}$. This wellestablished method allows for separation according to protein complex size rather than charge/mass ratio in the $\mathrm{BN}$ gradient ge ${ }^{58}$. Here, the $\mathrm{BN}$-gel showed a clear separation for solubilized cardiac membrane protein complexes. Of note, there may be differences in gradient gel mixture, BNE running conditions, and cutting of gel pieces. In order to achieve higher reproducibility with a similar resolution in protein complex separation, an alternative approach is size exclusion chromatography followed by mass spectrometry analysis ${ }^{106}$.

\subsubsection{Complexome Profiling analysis of native cardiac membranes}

Complexome profiling has been used successfully for the identification of new protein-protein interactions $^{74,87,89}$ or protein-assemblies and their intermediates ${ }^{89}$. It is also used to identify protein complex changes in patients with mitochondrial diseases ${ }^{107}$. Thus, complexome profiling is a suitable method for the analysis of complexes and protein-protein interactions of multiple proteins at the same time. The analysis of cardiac membranes from PLN WT and KO mouse ventricles revealed unexpected changes in migration profiles and protein abundances in macromolecular complexes. 
For quality control, mass calibration of soluble and membrane proteins established a linear correlation indicating a sufficient separation of proteins within the BN gel. Additionally, mitochondrial respiratory chain complex assemblies were detected as described previously ${ }^{74,89}$ without alterations between PLN WT and KO. This finding is in good agreement with normal heart rates in PLN KO mice with no reported mitochondrial defects ${ }^{36}$.

Analogous to SERCA2a regulation by PLN, the Na,K-ATPase activity is regulated by the muscle-specific micropeptide phospholemman (PLM) ${ }^{91}$. Remarkably, different states of the $\mathrm{Na}$,K-ATPase complex ${ }^{90}$ associated with PLM were captured by mass differences. This example shows the sensitivity, and resolution of this method, to distinguish between molecular complexes with different functional states and may, thus, allow detecting functional changes also in disease and therapeutic intervention models.

Most interestingly, as expected PLN and SERCA2a are tightly associated with increased abundance in the high molecular weight range. This finding is quite different from the observations for Na,K-ATPase and PLM complex distribution, suggesting different behaviors for plasma membrane and SR proteins, as well as a substantial PLN/SERCA2a organization in macromolecular complexes. Germline PLN ablation resulted in major alterations of these complexes, consistent with its functional significance in the heart ${ }^{36}$.

Furthermore, soluble proteins like protein phosphatases and kinases were identified, suggesting associations to membrane proteins. Protein phosphatase 1 (PP1) plays an essential role in calcium signaling and is targeted to RyR2 and PLN in the heart ${ }^{108,99,61}$. Here, not PP1, but its regulatory subunit PPP1R3A was abundant in macromolecular complexes. As the only detected regulatory subunit of PP1, it showed an overlap with RyR2 and the PLN/SERCA2a complexes. Alsina et al. could show that PPP1R3A targets PP1 not only to PLN but additionally to RyR ${ }^{61}$. The finding was supported by complexome profiling and revealed a previously unknown $\mathrm{SR} \mathrm{Ca}^{2+}$ cycling complex comprised of RyR2 and PLN/SERCA2a, and each together with PPP1R3A. It is interesting to note that PLN ablation led to a reduced abundance of RyR2 and SERCA2a and a near total loss of PPP1R3A in the high molecular weight complexes. Together with STED superresolution microscopy, our data may support a new physically interacting and functionally co-regulated signaling nanodomain termed ' $\mathrm{SR} \mathrm{Ca}{ }^{2+}$ cycling complex'61. It remains to be established whether this complex is organized by additional interactions or assembly factors.

Moreover, the cAMP-dependent protein kinase A (PKA), which phosphorylates PLN at Ser$16^{92}$, was detected in its inactive tetrameric and its catalytic active state ${ }^{93,94}$. PKA is targeted to substrates at different subcellular loci by A-kinase anchoring proteins (AKAPs) ${ }^{109,110}$. However, in our data PKA was not detected in macromolecular complexes, and AKAPs were not detected under these experimental conditions. The second kinase which phosphorylates PLN is the calcium/calmodulin-dependent protein kinase 2 (CaMK2). In addition to the 
predominant cardiac isoform $\mathrm{CaMK} 2 \delta^{95}$, CaMK2 $\alpha$ was detected. Both isoforms were abundant in macromolecular complexes, which is consistent with the reported CaMK2 $\delta$ targeting to SR membranes ${ }^{95}$. Additionally, CaMK2 signals at the high molecular weight are overlapping with the PLN/SERCA2a complex, which may present an additional constituent of the described SR $\mathrm{Ca}^{2+}$ cycling complex.

In general, these data show that complexome profiling is a valuable application for the analysis of cardiac macromolecular protein complexes. A conceptually new protein complex that includes PLN, SERCA2, and RyR2 has been identified, including PPP1R3A as a co-regulatory mechanism for PP1 targeting ${ }^{61}$.

\subsubsection{SLMAP proximity to PLN in cardiomyocytes}

In order to identify protein-protein interactions by complexome profiling, hierarchical clustering was applied to group proteins in clusters based on similarities in their migration pattern. Based on this clustering, the sarcolemmal membrane-associated protein (SLMAP) was identified as potentially interacting protein in the PLN/SERCA2a complex. PLN ablation showed a higher abundance of SLMAP in macromolecular complexes and an overall increased signal. Immunoblotting confirmed a significant increase in SLMAP protein expression (SLMAP1, SLMAP3). Interestingly, all SLMAP isoforms were abundant in the macromolecular complexes suggesting a functional role.

SLMAPs are targeted to different subcellular locations depending on their transmembrane tailanchored domain ${ }^{81,96}$. In contrast to earlier reports ${ }^{96}$, SLMAP was not localized in the sarcolemma and the T-tubular system but was present in SR membranes along transversal Zlines in ventricular cardiomyocytes. Surprisingly, neither changes in SLMAP localization nor its cell-wide distribution were apparent in PLN KO cardiomyocytes. In WT cardiomyocytes, SLAMP was detected immediately adjacent to PLN rich SR domains, and coimmunoprecipitation experiments showed co-elution of all SLMAPs. These data suggested a proximity-based local association of SLMAP and PLN, which may contribute to a functional signaling complex in the SR membrane.

\subsubsection{SLMAP expression changes in cardiac hypertrophy}

Cardiac hypertrophy increases the risk for congestive heart failure ${ }^{111}$ and is characterized by reduced SERCA2a expression and thus dysregulated $\mathrm{Ca}^{2+}$ signaling in cardiomyocytes ${ }^{112}$. Here, the $\mathrm{Ca}^{2+}$ transport proteins RyR2 and SERCA2a showed decreased expression, whereas PLN showed no changes 4 weeks after transverse aortic constriction (TAC). In contrast, SLMAP isoforms were significantly increased at protein levels in ventricular 
cardiomyocytes isolated 4 weeks after TAC. This confirms previous findings, showing that SLMAP3 protein expression was significantly increased 30 days after TAC in mice ${ }^{98}$. However, in human dilated cardiomyopathy ventricles, a decreased expression of SLMAP was reported $^{86}$. This finding differs from the increased SLMAP expression we found in mice with induced left ventricular hypertrophy and might be explained by the advanced end-stage DCM post-mortem phenotype of human ventricles.

Additionally, 4 weeks after TAC, only SLMAP2 protein expression was significantly increased in PLN KO hearts compared to Sham WT hearts. However, SLMAP1 and SLMAP3 protein levels in TAC PLN KO were equal to Sham WT. This suggests that induced ventricular hypertrophy in PLN KO may decrease the protein expression of SLMAP1 and SLMAP3 since the basic level of SLMAP in PLN KO hearts is significantly increased compared to WT. These results thus need to be interpreted with caution due to the small group size of PLN KO mice after TAC $(n=2)$.

\subsubsection{SLMAP expression in human iPSC-derived cardiomyocytes}

SLMAP transcriptional expression in human induced pluripotent stem cells was found to significantly increase during cardiomyocyte differentiation (day 12 vs. day 40$)^{113}$. We made similar observations during iPSC-cardiomyocyte differentiation and maturation from day 60 to day 90. The increasing expression of SLMAP during maturation might also affect the localization in iPSC-CM. SLMAP localization was observed as subcellular clustered in 60 days old iPSC-CM. This contrasts with the SLMAP distribution along Z-lines in adult mouse VCMs. Thus, SLMAP localization may change due to maturation and increased expression levels ${ }^{113}$. Importantly, in iPSC-CM, SLMAP was localized in nanometric proximity to PLN and SERCA2a shown by STED nanoscopy (Figure 21).

In summary, SLMAP was identified as a potential interacting protein by migration cluster analysis in complexome profiling. SLMAP was further identified as PLN interacting protein by co-immunoprecipitation and showed a proximal association with PLN in mouse VCM and human iPSC-CM by STED superresolution microscopy. In PLN KO and TAC operated WT mice, SLMAP protein expression was found to be significantly increased.

It remains to be determined if the differently spliced SLMAP TM domains mediate the PLN binding. Furthermore, SLMAP3 has a unique N-terminal FHA domain, which can interact with other proteins in a phospho-dependent manner ${ }^{114}$. Hence, SLMAP3 may also interact with PLN through the FHA domain in a phospho-dependent manner.

Together, these data suggest a proximity-based local association of SLMAP and PLN, which may contribute to a functional signaling complex in the SR membrane. 


\section{$4 \quad$ PLN targeted APEX2 proximity labeling}

\subsection{Introduction}

As mentioned before, protein-protein interactions are involved in all kinds of cellular processes. Standard methods for the identification of protein-protein interactions frequently use in vitro and non-physiological conditions. Recently, new methods have been introduced for the proximity labeling of proteins in living cells. These techniques are based on exogenous enzymes that generate reactive labels that enable protein isolation and identification by mass spectrometry. For example, biotin was exploited as a protein label to isolate with avidin or streptavidin specifically. Once it has been attached covalently, biotinylated proteins can be captured under stringent lysis and wash conditions resulting in a higher purity of isolated proteins.

For proximity-dependent biotin identification (BiolD) ${ }^{66,67}$, a biotin ligase is bound to a baitprotein for proximity labeling. Upon cellular expression, it generates reactive biotin and fuses it to proximal proteins with a labeling period of hours. This results in an accumulation of labeled proteins at a distance of approximately $10 \mathrm{~nm}$ to the bait protein ${ }^{115}$.

A more rapid way to label proximal proteins is the APEX proximity labeling. It can also be applied in living cells and is bound to the bait protein. APEX is an engineered ascorbate peroxidase from soybean, which creates by its enzymatic activity short-lived radicals reacting with tyrosine and other electron-rich amino acids as tryptophan, cysteine, and histidine ${ }^{68}$. The APEX reaction creates not a history of interacting proteins like BioID but rather a snapshot by a short reaction pulse of $1 \mathrm{~min}^{54}$. The improved $2^{\text {nd }}$ generation variant, APEX2, improved its reactivity and can be applied at lower concentrations ${ }^{65}$. Previously, APEX2 was successfully used for mapping proteomes in the intermembrane space in mitochondria ${ }^{68}$, cilia $^{70}$, and also for dynamic approaches of G-protein coupled receptors upon stimulation ${ }^{71}$.

For screening potential novel interaction partners of PLN, a fusion construct of APEX2 and canine phospholamban was designed (Figure 22A,B). To allow for the identification of interaction partners from the cytosol, APEX2 was tagged N-terminally to PLN. A flexible GS linker maintains PLN interactions with endogenous proteins. The N-terminal epitope tag V5 was used for detection in immunoassays. Following the expression with APEX2-PLN and the incubation with biotin-phenol (BP), the cells were exposed to hydrogen peroxide $\left(\mathrm{H}_{2} \mathrm{O}_{2}\right)$ to initiate the enzymatic reaction ${ }^{54}$ (Figure 22C). BP is converted to the short-lived biotin-phenoxyl radical, which covalently labels proteins in nanometric proximity in a range from $<20 \mathrm{~nm}^{116,117,118}$. As spatial sarcoplasmic reticulum targeted control, a truncation of PLN missing amino acids 1 to 29 was used (Figure 22A,B). Hence, only the transmembrane domain of PLN was fused to APEX2, resulting in APEX2-PLN $\Delta(1-29)$. 
This work showed that APEX2-PLN could be targeted to subcellular locations in cardiomyocytes for proximity live labeling. For a ratiometric proteomic approach, SILAC was established for quantification in neonatal rat cardiomyocytes (NRCMs), and potential PLN protein interactors were labeled. This approach has potentially important implications for further understanding of the regulation of $\mathrm{SR} \mathrm{Ca}^{2+}$ uptake in the heart.

A
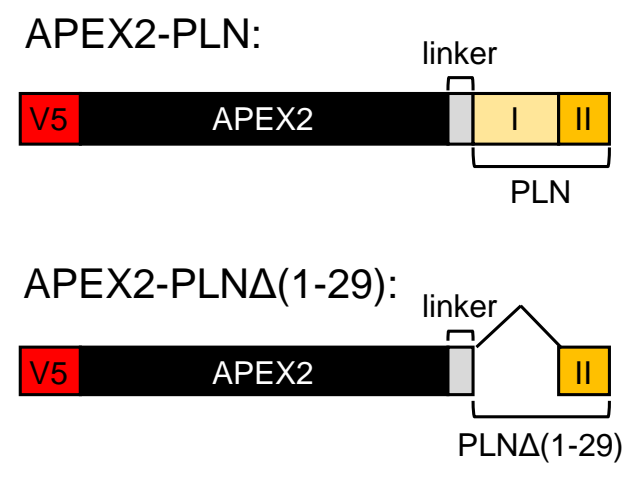

B

\section{APEX2-PLN}
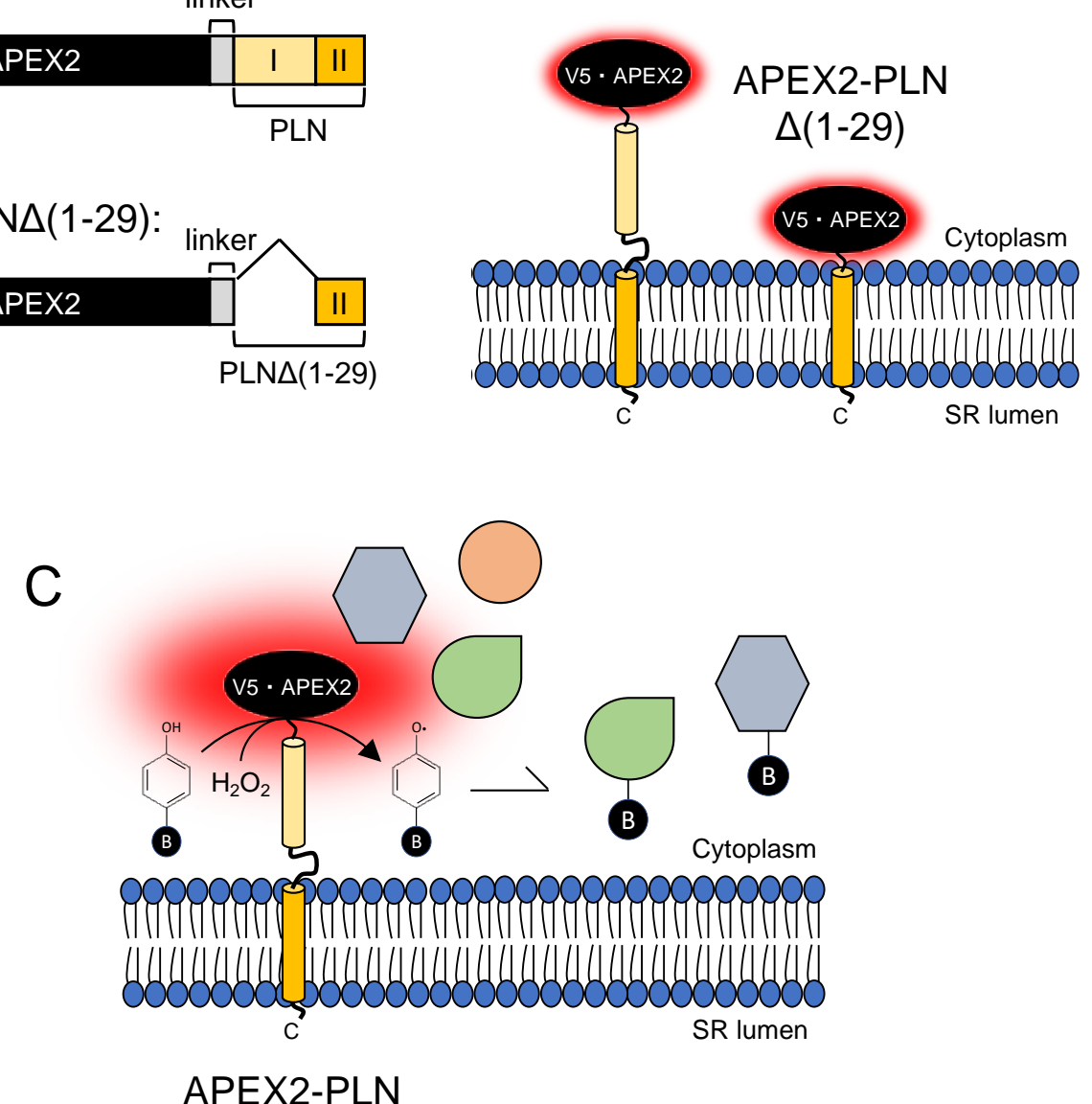

Figure 22 Proximity proteomics of canine phospholamban for the identification of novel interaction partners

A, For proximity proteomics, PLN was tagged N-terminally with the epitope tag V5 and the ascorbate peroxidase APEX2 (APEX2-PLN). APEX2 was separated by a GS linker to enhance flexibility and enable PLN protein interactions. As spatial sarcoplasmic reticulum (SR) targeted control, only the PLN transmembrane domain was $\mathrm{N}$-terminally tagged by V5 and APEX2 (APEX2-PLNA(1-29)). B, APEX2 expression constructs are located in the SR membrane upon expression, while the PLN cytosolic domain and V5-APEX2 face the cytosol. C, Scheme of PLN proximity biotinylation in living cells. APEX2 generates the biotin-phenoxyl radical (red) and covalently labels proteins in close proximity $(<20 \mathrm{~nm})$ with biotin (B). Living cells were incubated with $500 \mu \mathrm{M}$ biotin-phenol (BP) for $30 \mathrm{~min}$, followed by $1 \mathrm{~min}$ exposure of $1 \mathrm{mM} \mathrm{H}_{2} \mathrm{O}_{2}$. 


\subsection{Results}

\subsubsection{APEX2-PLN proximity labeling in HEK293A cells}

To investigate the proximity of PLN by APEX2 proximity labeling, the used APEX2 constructs were tested to confirm the correct cellular localization in HEK293A. Therefore, the cells were plasmid co-transfected with APEX2-PLN or APEX2-PLN $\triangle(1-29)$ and KDEL-Myc expressing plasmid used as an endoplasmic reticulum marker. Confocal co-immunofluorescence imaging showed a robust expression and co-localized with the ER marker in the perinuclear and peripheral ER.

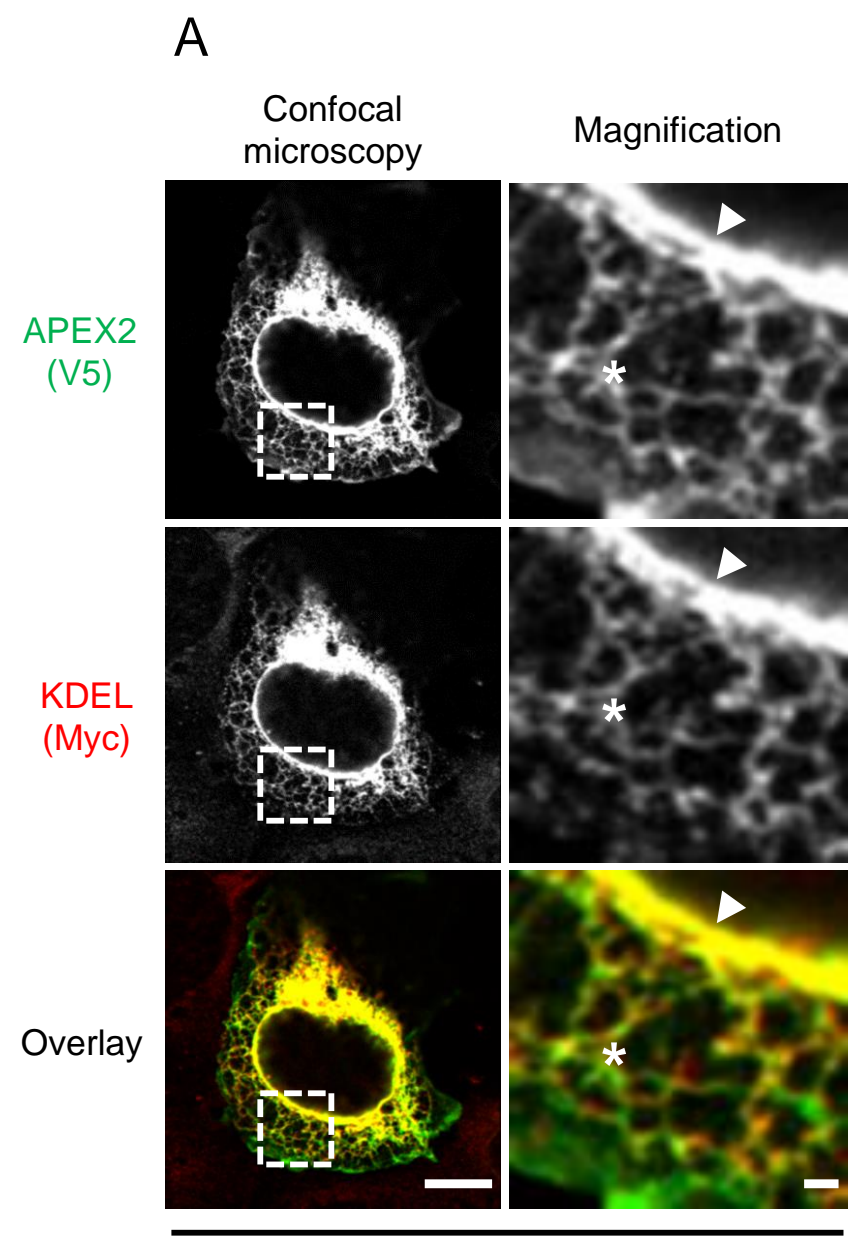

APEX2-PLN
B

Confocal microscopy
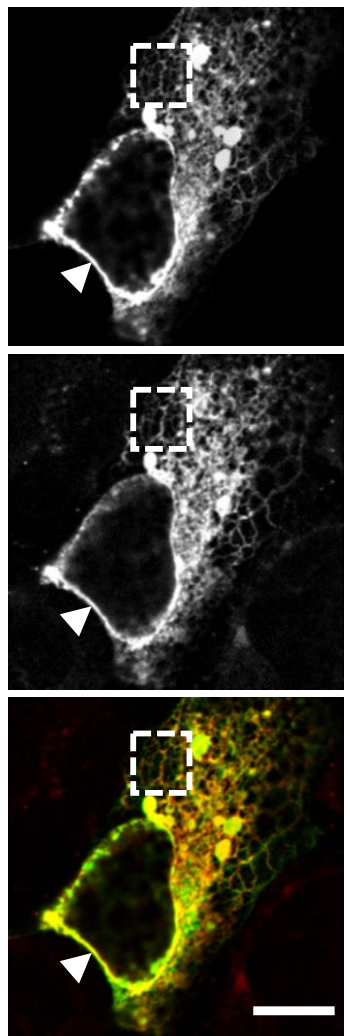

Magnification
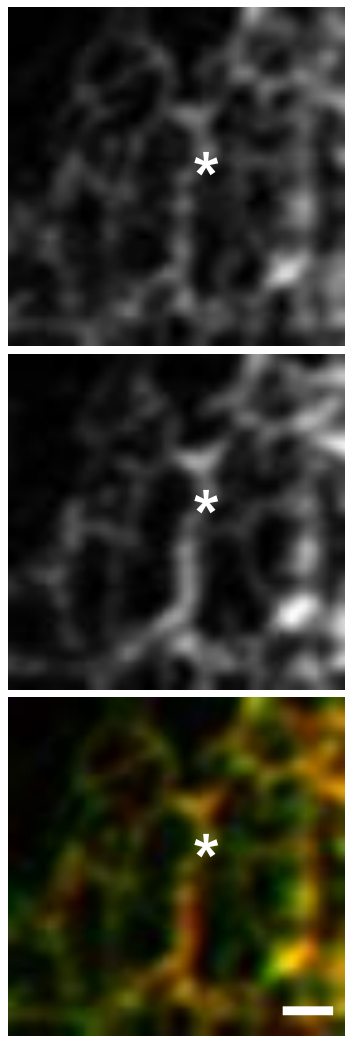

APEX2-PLN $\Delta(1-29)$

Figure 23 Confocal co-immunofluorescence imaging of APEX2-PLN and APEX2-PLNA(1-29) in HEK293A cells

HEK293A cells were co-transfected with either APEX2-PLN (A) or APEX2-PLNA(1-29) (B) and KDELMyc plasmid used as endoplasmic reticulum (ER) marker. After $24 \mathrm{~h}$, samples were prepared and stained for co-immunofluorescence with V5 and Myc antibodies. Confocal imaging (Zeiss LSM 880, 63x/1.4 Oil DIC objective) shows an expression of APEX2 constructs and co-localization with an ER 
marker (KDEL peptide) in the peripheral ER (asterisk) or the perinuclear ER (arrowhead). Dashed boxes indicate areas of magnified views. Scale bars: Confocal microscopy $10 \mu \mathrm{m}$; Magnification $1 \mu \mathrm{m}$.

To test the catalytic APEX2 activity, plasmid transfected HEK293A cells were treated for 30 min with $500 \mu \mathrm{M}$ biotin-phenol at $37{ }^{\circ} \mathrm{C}$ and $1 \mathrm{mM} \mathrm{H}_{2} \mathrm{O}_{2}$ at room temperature (Figure 24). Immunoblots showed induction of biotinylation dependent on APEX2 transfected cells, biotinphenol, and $\mathrm{H}_{2} \mathrm{O}_{2}$. Omitting biotin-phenol or $\mathrm{H}_{2} \mathrm{O}_{2}$ showed only a few endogenously biotinylated proteins.

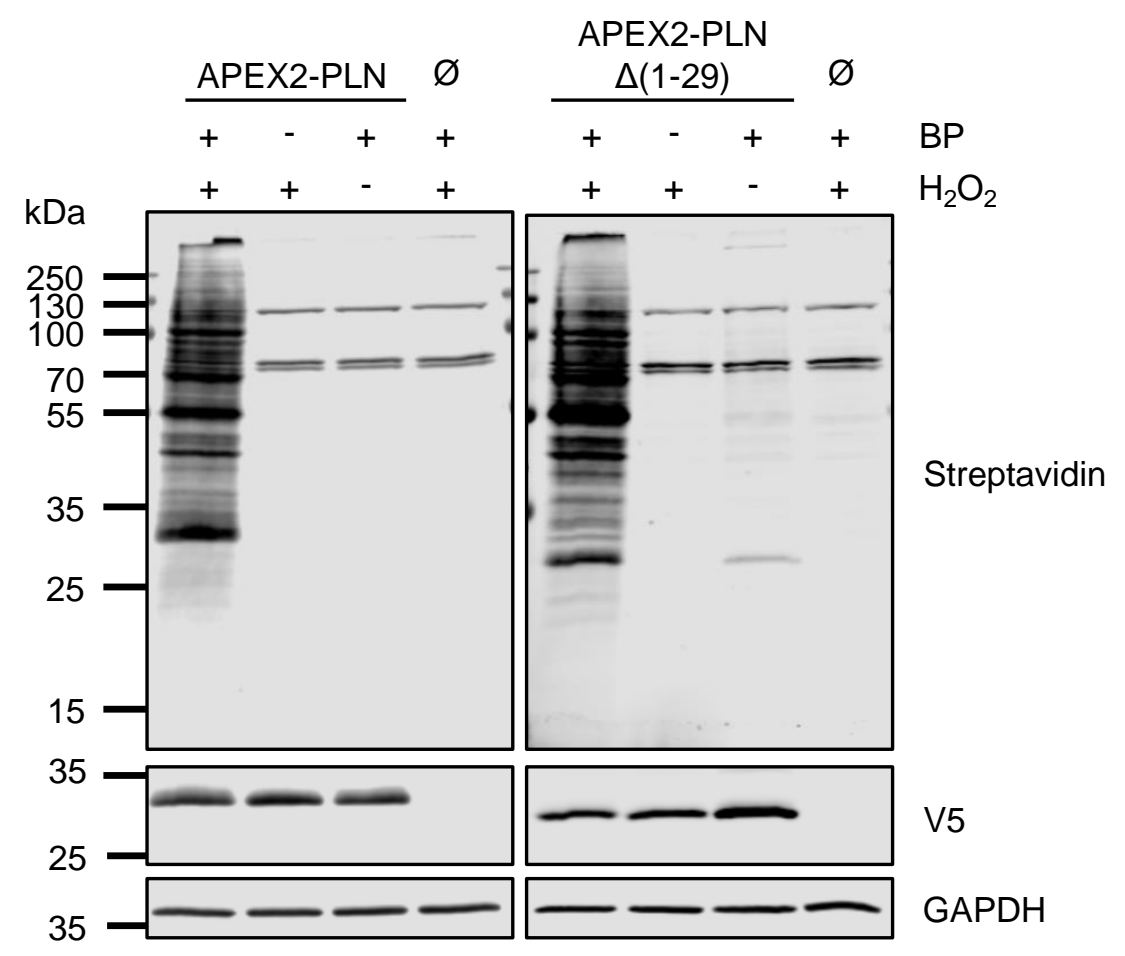

Figure 24 PLN based proximity labeling in transfected HEK293A cells

HEK293A cells were plasmid transfected with APEX2-PLN or APEX2-PLNA(1-29). After $24 \mathrm{~h}$ of expression, proximity labeling was initiated in living cells with $500 \mu \mathrm{M}$ biotin-phenol (BP) for $30 \mathrm{~min}$, followed by $1 \mathrm{mM} \mathrm{H} 2 \mathrm{O} 2$ for $1 \mathrm{~min}$. Biotinylated proteins were visualized by immunoblotting using streptavidin IRDye 680RD. Transfection controls (Ø) and omitting BP or H2O2 (-) show only endogenous biotinylated proteins. V5 antibody shows the expression of APEX2 constructs, GAPDH was used as a loading control.

Next, we tested the enrichment of biotinylated proteins by affinity purification using avidinagarose beads. Varying the elution conditions using biotin and SDS ${ }^{119}$ for different incubation times and temperatures, we found the elution with $2 \mathrm{mM}$ biotin and $2 \%$ SDS for $15 \mathrm{~min}$ at RT and 15 min at $70{ }^{\circ} \mathrm{C}$ as most efficient (Figure 25). 
In general, APEX2-PLN and APEX2-PLNA(1-29) plasmid constructs could be expressed transiently in HEK293A cells. They were localized to the ER as expected and APEX2 was functionally active shown by the biotinylation reaction. The enrichment of biotinylated proteins was achieved by established protocols.

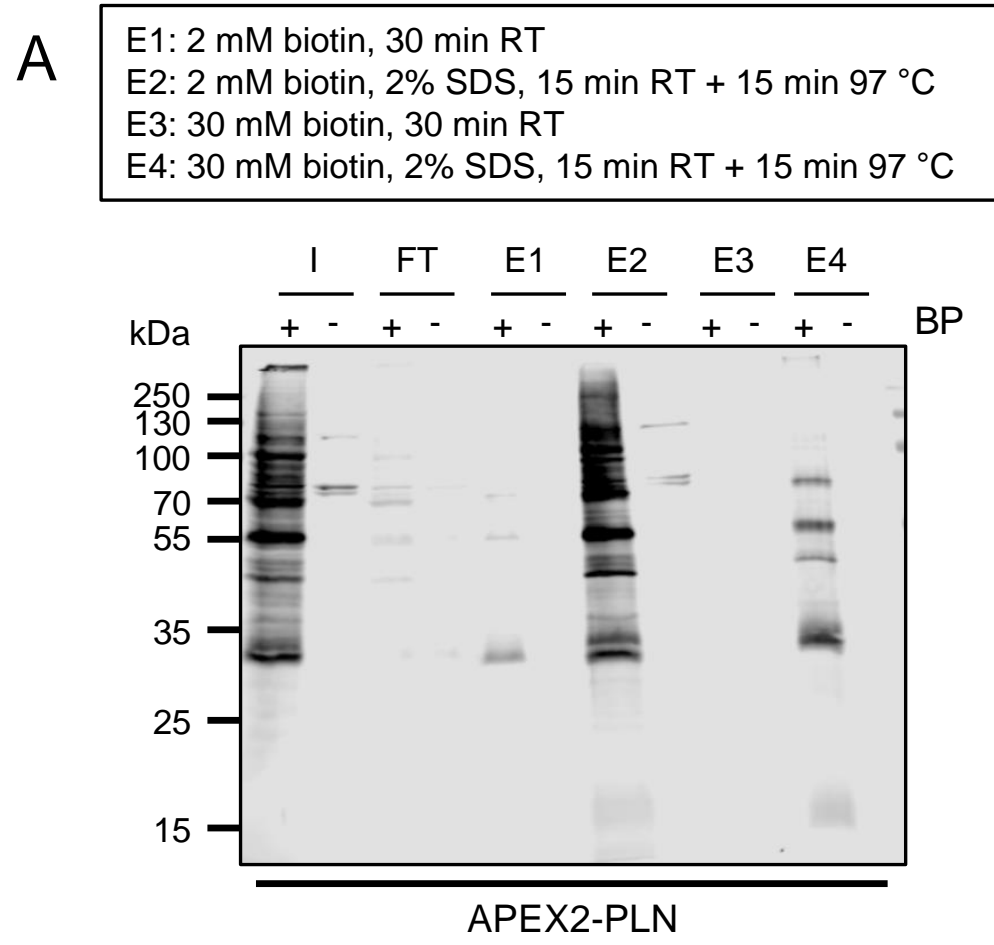

\begin{tabular}{l|l|}
\hline B & E2.1: $2 \mathrm{mM}$ biotin, $2 \% \mathrm{SDS}, 30 \mathrm{~min} \mathrm{RT}$ \\
E2.2: $2 \mathrm{mM}$ biotin, $2 \% \mathrm{SDS}, 15 \mathrm{~min} \mathrm{RT}+15 \mathrm{~min} 70^{\circ} \mathrm{C}$ \\
E2.3: $2 \mathrm{mM}$ biotin, $2 \% \mathrm{SDS}, 15 \mathrm{~min} \mathrm{RT}+15 \mathrm{~min} 97^{\circ} \mathrm{C}$
\end{tabular}

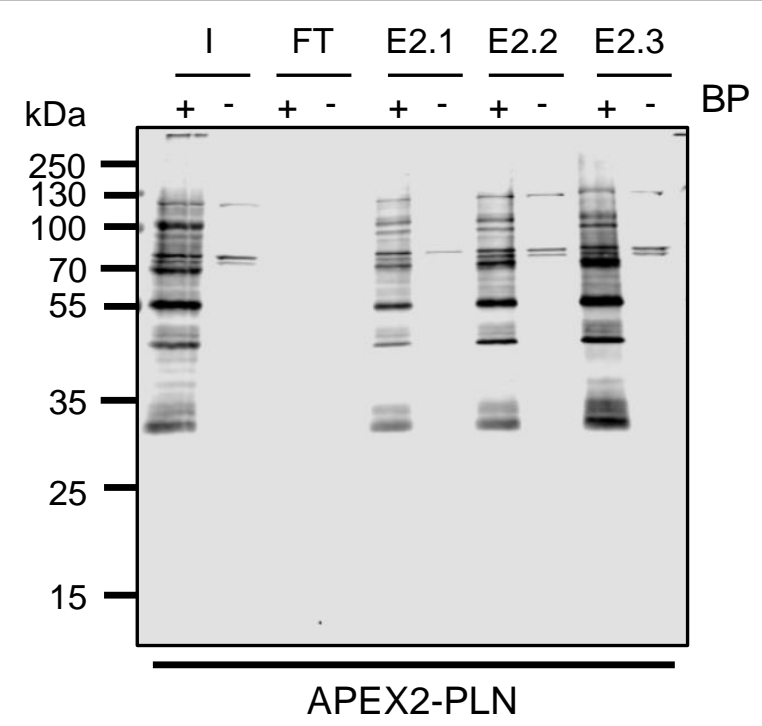

Figure 25 Affinity purification optimization of biotinylated proteins after proximity labeling in HEK293A 
APEX2-PLN plasmid transfected HEK293A cells were treated with (+) or without (-) biotin-phenol (BP) and $\mathrm{H} 2 \mathrm{O} 2$ to induce biotinylation. After cell lysis, biotinylated proteins were enriched by affinity purification with monomeric avidin. A, Different elution conditions were used as indicated, and $2 \mathrm{mM}$ biotin and $2 \%$ SDS (E2) were found to be most efficient. Input (I) and flow-through (FT) were shown representative from experiment E1. B, Elution condition E2 was further optimized, testing different incubation times and temperatures as indicated. Input and flow through were shown representative from experiment E2.1. Biotinylated proteins were detected by immunoblotting using streptavidin IRDye 680RD.

\subsubsection{Neonatal rat cardiomyocyte cell model}

Since PLN is mostly expressed in the heart ${ }^{28,26}$, neonatal rat cardiomyocytes (NRCMs) were used as a cell model for the PLN targeted APEX2 proximity live labeling. NRCMs were isolated fresh from neonatal rat hearts and provide week-long observation periods, spontaneous beating, and maturation of the cell population ${ }^{120}$. To verify the localization of endogenous PLN and SERCA2a, NRCMs were labeled for co-immunofluorescence microscopy. PLN and SERCA2a showed overlapping signals with a reticular structure, whereas PLN showed additionally perinuclear signals confirming previous findings ${ }^{121,122}$ (Figure 26). 


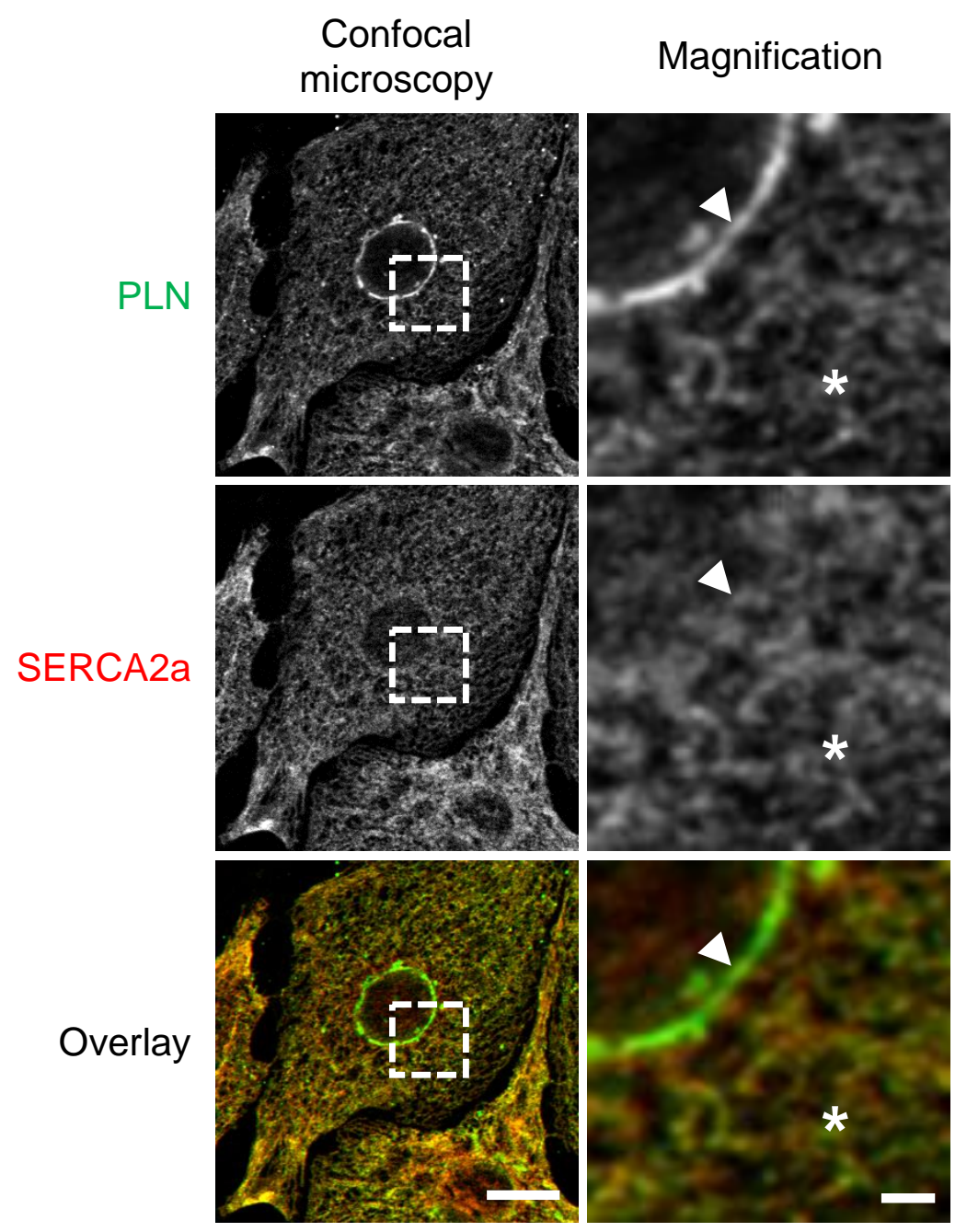

Figure 26 Confocal co-immunofluorescence imaging of endogenous PLN and SERCA2a in NRCMs

NRCMs were prepared for co-immunofluorescence staining after six days of cultivation. Confocal coimmunofluorescence imaging showed robust expression of PLN and SERCA2a and overlapping signals in reticular structures (asterisk). Additionally, PLN showed enriched signals in the perinuclear envelope independent of SERCA2a (arrowhead). Dashed boxes indicate areas of magnified views. Scale bars: Confocal microscopy $10 \mu \mathrm{m}$; Magnification $1 \mu \mathrm{m}$.

First, plasmid transfections using Lipofectamine 3000 were tested to target the APEX2 constructs for expression in NRCMs. Co-immunofluorescence imaging of V5 and endogenous SERCA2a showed peripheral SR localizations for both constructs confirming the expected ER distribution (Figure 27). 


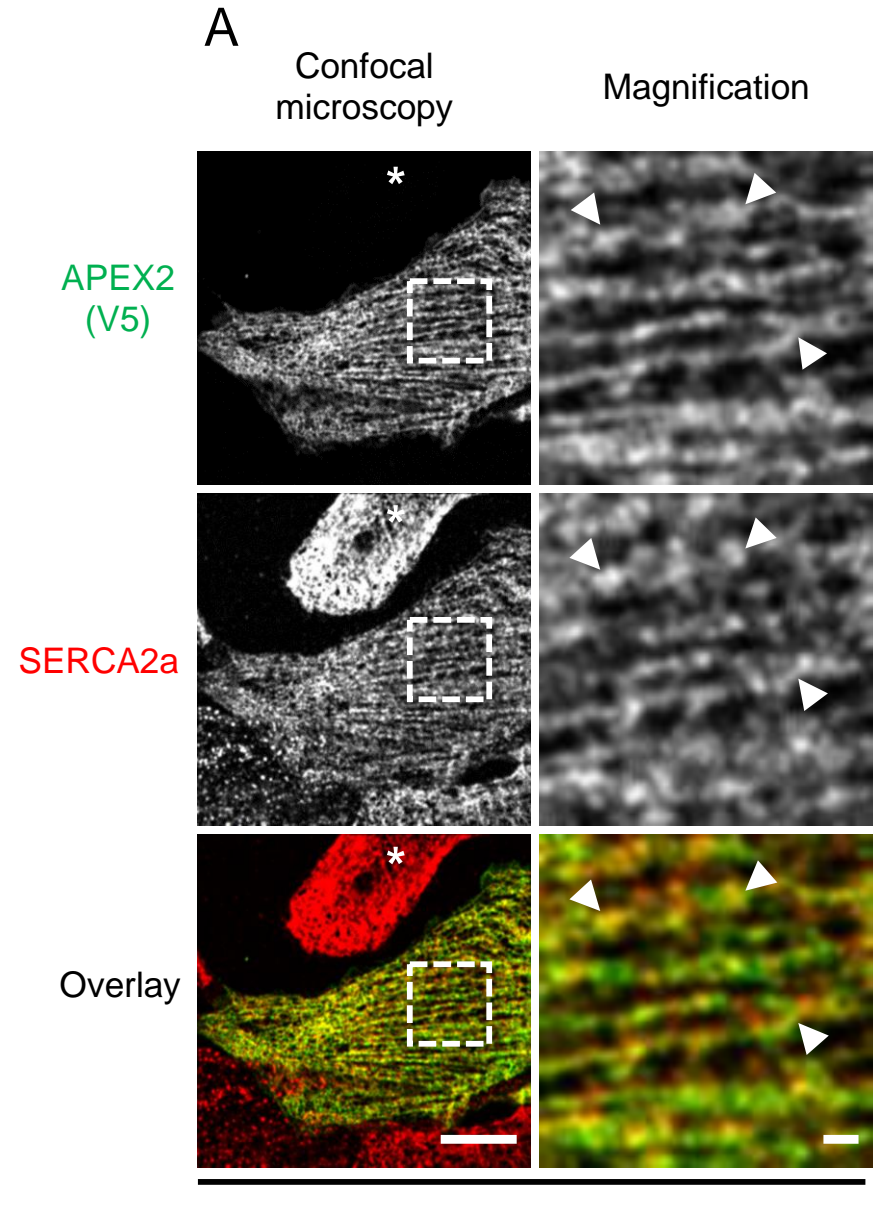

APEX2-PLN
B Confocal
microscopy
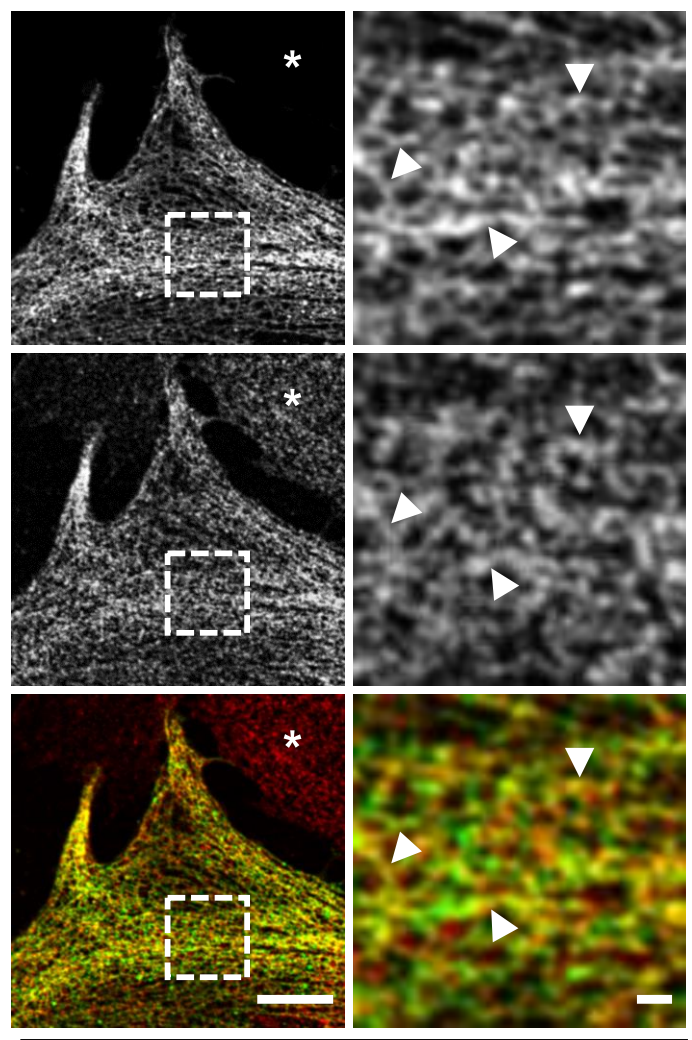

APEX2-PLN $\Delta(1-29)$

Figure 27 Confocal co-immunofluorescence imaging of APEX2-PLN and APEX2-PLNA(1-29) in NRCMs

Six days after cultivation, primary NRCMs were plasmid transfected with APEX2-PLN or APEX2PLNA(1-29). After $24 \mathrm{~h}$ of expression, confocal co-immunofluorescence imaging showed construct expression of APEX2-PLN (A) and APEX2-PLNA(1-29) (B). Co-localization with SERCA2a identified a peripheral SR localization (arrowheads). Asterisks show un-transfected cells without V5 signal proofing antibody specificity. Dashed boxes indicate areas of magnified views. Scale bars: Confocal microscopy $10 \mu \mathrm{m}$; Magnification $1 \mu \mathrm{m}$.

Furthermore, the catalytic APEX2 activity in plasmid transfected NRCMs was tested. NRCMs were treated with $\mathrm{BP}$ and $\mathrm{H}_{2} \mathrm{O}_{2}$ and showed induced biotinylation of proteins detected by labeled streptavidin in immunoblot (Figure 28A). Additionally, V5 signals show oligomeric bands $>250 \mathrm{kDa}$ (Figure 28B).

Taken together, APEX2-PLN and APEX2-PLN $\Delta(1-29)$ plasmid constructs could also be expressed transiently in NRCMs. The APEX2 proteins were localized to the SR similar to the endogenous SERCA2a distribution and showed functional activity by inducing biotinylation of 
proteins. Important to note is that the transfection efficiency using lipofectamine was very low (1-2\%) in NRCMs compared to HEK293A cells (data not shown).

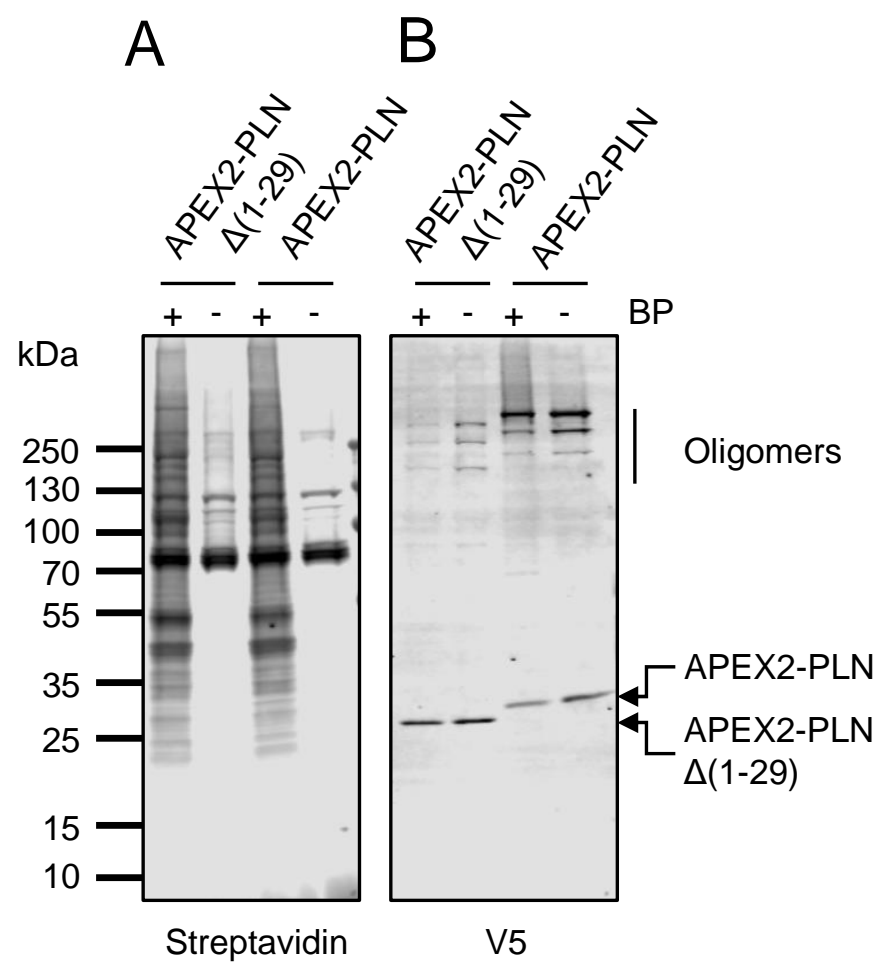

Figure 28 PLN specific proximity labeling in plasmid transfected NRCMs

NRCMs were plasmid transfected with APEX2-PLNA(1-29) or APEX2-PLN. After $24 \mathrm{~h}$ of expression, living cells were treated with (+) or without (-) $500 \mu \mathrm{M}$ biotin-phenol (BP) and $1 \mathrm{mM} \mathrm{H} 2 \mathrm{O} 2$ to induce biotinylation. Immunoblot showed biotinylated proteins by streptavidin IRDye 680RD (A) and V5 antibody expression of APEX2 constructs (B) on the same blot. Omitting BP shows only endogenous biotinylated proteins. V5 tagged APEX2 constructs show monomeric and oligomeric signals (>250 kDa).

\subsubsection{Ratiometric proximity proteomics in NRCMs}

In order to identify quantitatively enriched proteins labeled in the proximity of PLN, the use of a ratiometric labeling strategy was desirable. For this kind of experiment, the stable isotope labeling of amino acids in cell culture (SILAC) is often used as a 'gold standard' $123,68,54$. As Feger et al. reported, NRCMs were SILAC labeled previously for five days, resulting in an isotope abundance of $\sim 40 \%^{124}$. To test the timescale until $95 \%$ of the proteins are SILAC labeled in primary isolated NRCMs, cells were cultivated up to 20 days in radiolabeled amino acids containing medium. NRCMs were lysed at different time points, trypsin digested, and subjected to mass spectrometry. Incorporation of at least $95 \%$ of labeled arginine and lysine was achieved after 13 days of cultivation (Figure 29A). Parallel immunoblot experiments showed a decreased protein expression of PLN and SERCA2a over a cultivation period of 
days (Figure 29B). This experiment was carried out together with Jonas Peper (Department of Cardiology and Pneumology, University Medical Center Göttingen).

Due to decreased protein expression of PLN and SERCA2a during cultivation, the earliest time point of sufficient SILAC incorporation was chosen, namely 13 days.

A

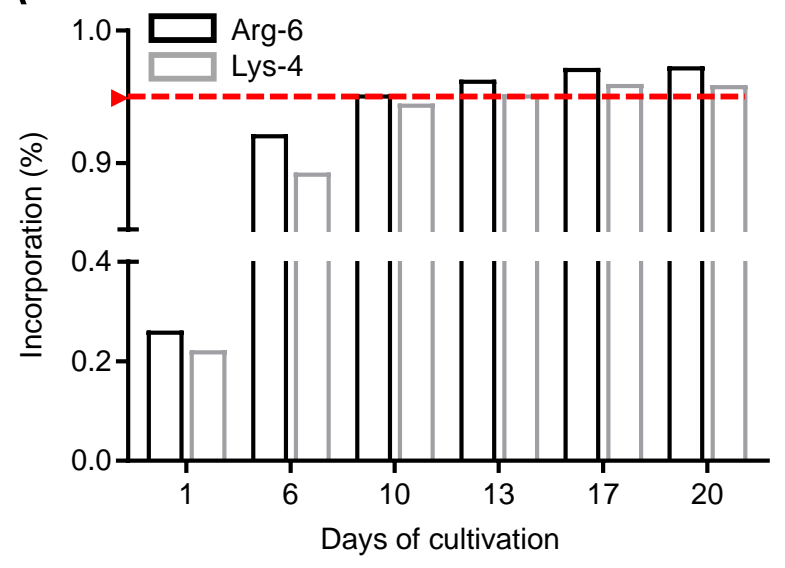

$\mathrm{B}$

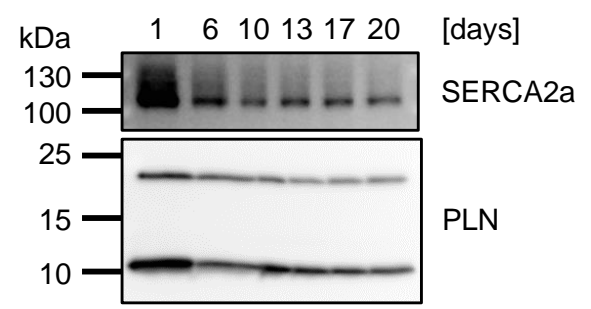

Figure 29 Stable isotope labeling of amino acids in cell culture (SILAC) incorporation in NRCMs NRCMs were cultivated over 20 days in medium containing radiolabeled L-arginine (Arg-6) and L-lysine (Lys-4) and harvested at the indicated time points. A, Mass spectrometry (LC-MS/MS) quantified incorporation of $>95 \%$ after 13 days of cultivation (red line). $n=1$. B, Immunoblot analysis showed a decreased protein expression of PLN and SERCA2a over the cultivation period.

\subsubsection{Adenoviral transduction for proximity labeling in NRCMs}

To achieve a sufficient expression of APEX2-PLN and APEX2-PLN $\Delta(1-29)$ for proximity labeling in NRCMs, APEX2-PLN and APEX2-PLN $\Delta(1-29)$ constructs were cloned into adenoviral expression plasmids for the generation of adenoviral particles (serotype V; Ad5) (Figure 5). Adenoviral particles were custom made by Sirion Biotech (Martinsried, Germany). In order to test the expression and the optimal ratio of adenoviral particles per cell, different concentrations of adenoviral particles were tested, namely multiplicity of infection (MOI). After $48 \mathrm{~h}$ of adenoviral expression, fluorescence imaging showed increased eGFP signals, whereas, at MOI 100, the signal dropped due to increased cell death (Figure 30A). Also, eGFP mRNA levels and protein levels show increased expression with increasing $\mathrm{MOI}$ (Figure 30B,C). Importantly, levels of mRNA and protein expression between APEX2 constructs were comparable, allowing the same $\mathrm{MOI}$ for transduction. The $\mathrm{MOI}$ of 10 was detected as the highest least cytotoxic concentration of adenoviral particles determined by microscopy (data not shown). 

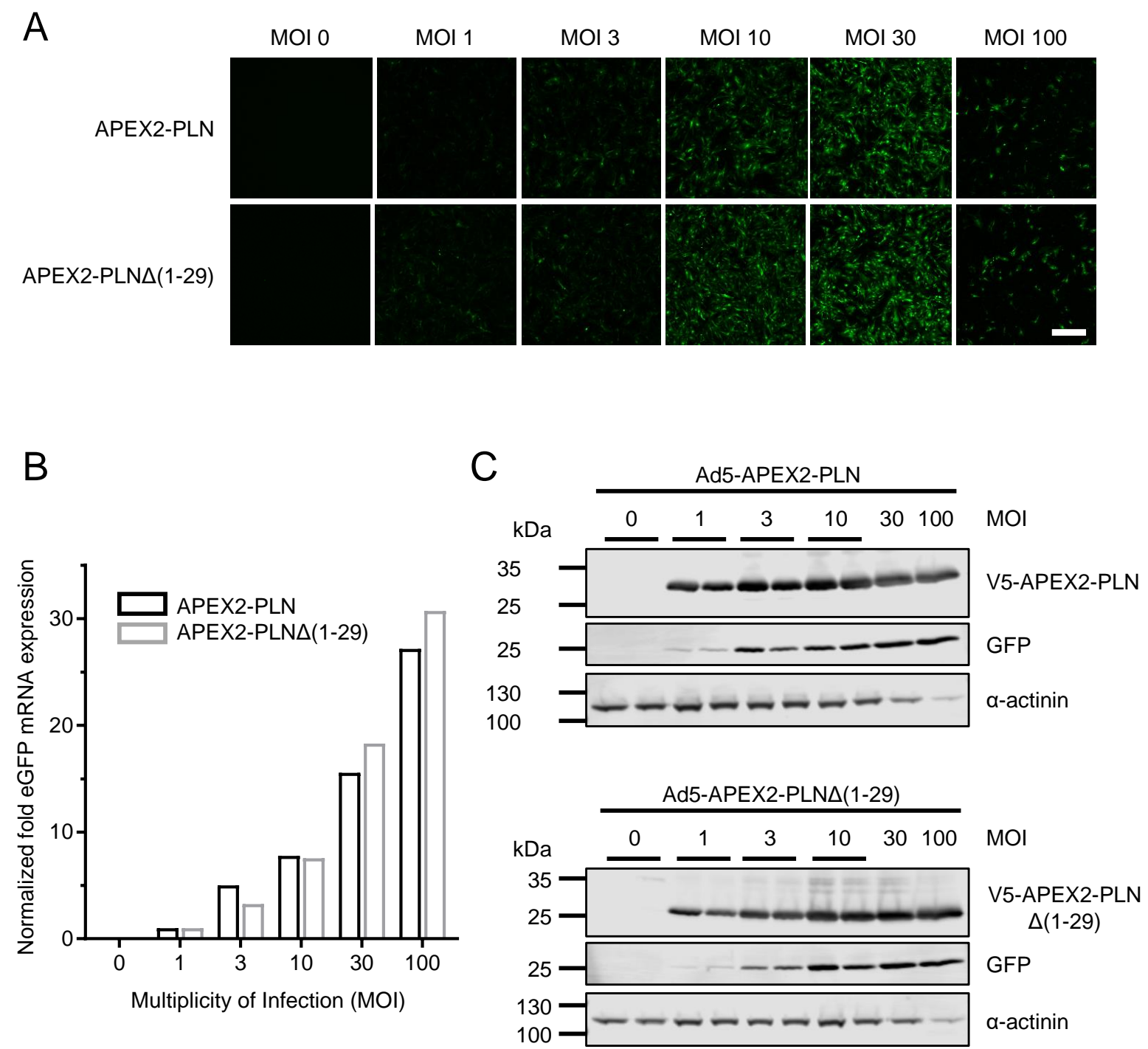

Figure 30 Analysis of adenoviral particle concentrations for NRCM transduction

NRCMs were transduced for $48 \mathrm{~h}$ with different concentrations of adenoviral particles (multiplicity of infection, MOI) to determine the optimal APEX2-PLN and APEX2-PLNA(1-29) expression. A, Fluorescence microscopy monitored eGFP marker protein expression increased with the viral particle concentration (5x objective, AxioVert Zeiss). MOI 100 showed lower eGFP signals due to increased cell death. Scale bar: $500 \mu \mathrm{m}$. B, qRT-PCR experiments determined equal eGFP mRNA expression levels using same adenoviral concentration in APEX2-PLN and APEX2-PLNA(1-29) transduced NRCMs. C, Immunoblots showed increased protein expression of V5-APEX2 constructs and eGFP with increasing $\mathrm{MOI}$ of adenoviral transduced NRCM lysates. $\alpha$-actinin was used as a loading control.

Additionally, confocal co-immunofluorescence imaging confirmed APEX2 construct protein expression in the same cellular compartment as SERCA2a after adenoviral transduction in NRCMs (Figure 31). 
To summarize, adenoviral constructs for APEX2-PLN and APEX2-PLN $\Delta(1-29)$ were successfully targeted into NRCMs and optimized for transduction. Confocal microscopy showed cellular localization similar to SERCA2a signals.

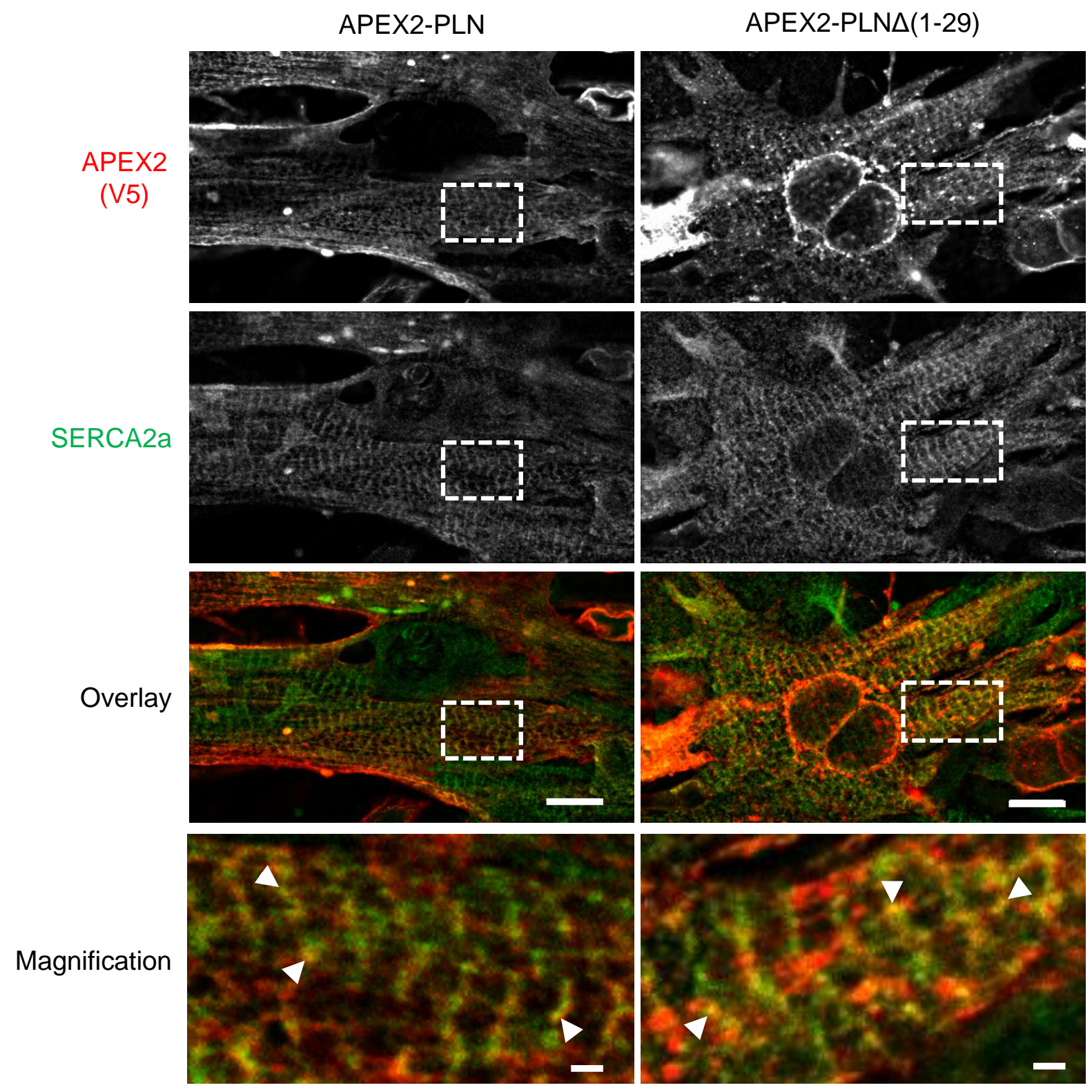

Figure 31 Confocal co-immunofluorescence imaging of adenoviral APEX2-PLN and APEX2-

\section{$\mathrm{PLN} \Delta(1-29)$ expression in NRCMs}

NRCMs were cultured for 15 days and transduced with Ad5-APEX2-PLN or Ad5-APEX2-PLNA(1-29) with MOI 10 for $48 \mathrm{~h}$. Cells were fixed, and adenoviral APEX2 constructs were co-stained for V5 (red) and SERCA2a (green). Confocal microscopy showed a striated distribution of APEX2 constructs and partial overlap with SERCA2a signals (arrowheads). Dashed boxes indicate areas of magnified views. Scale bars: $10 \mu \mathrm{m}$; Magnification $1 \mu \mathrm{m}$. 


\subsubsection{PLN specific ratiometric APEX2 proximity labeling in NRCMs}

For the final experiment, NRCMs were cultivated in total for 15 days in radiolabeled amino acids containing medium, including two days of adenoviral expression using MOI 10 (Figure 32A). After induced biotinylation, APEX2 transduced NRCMs showed increased biotinylation of proteins, whereas the transduction control eGFP showed only endogenously biotinylated proteins (Figure 32B). V5 signals showed a similar expression for both APEX2 proteins.
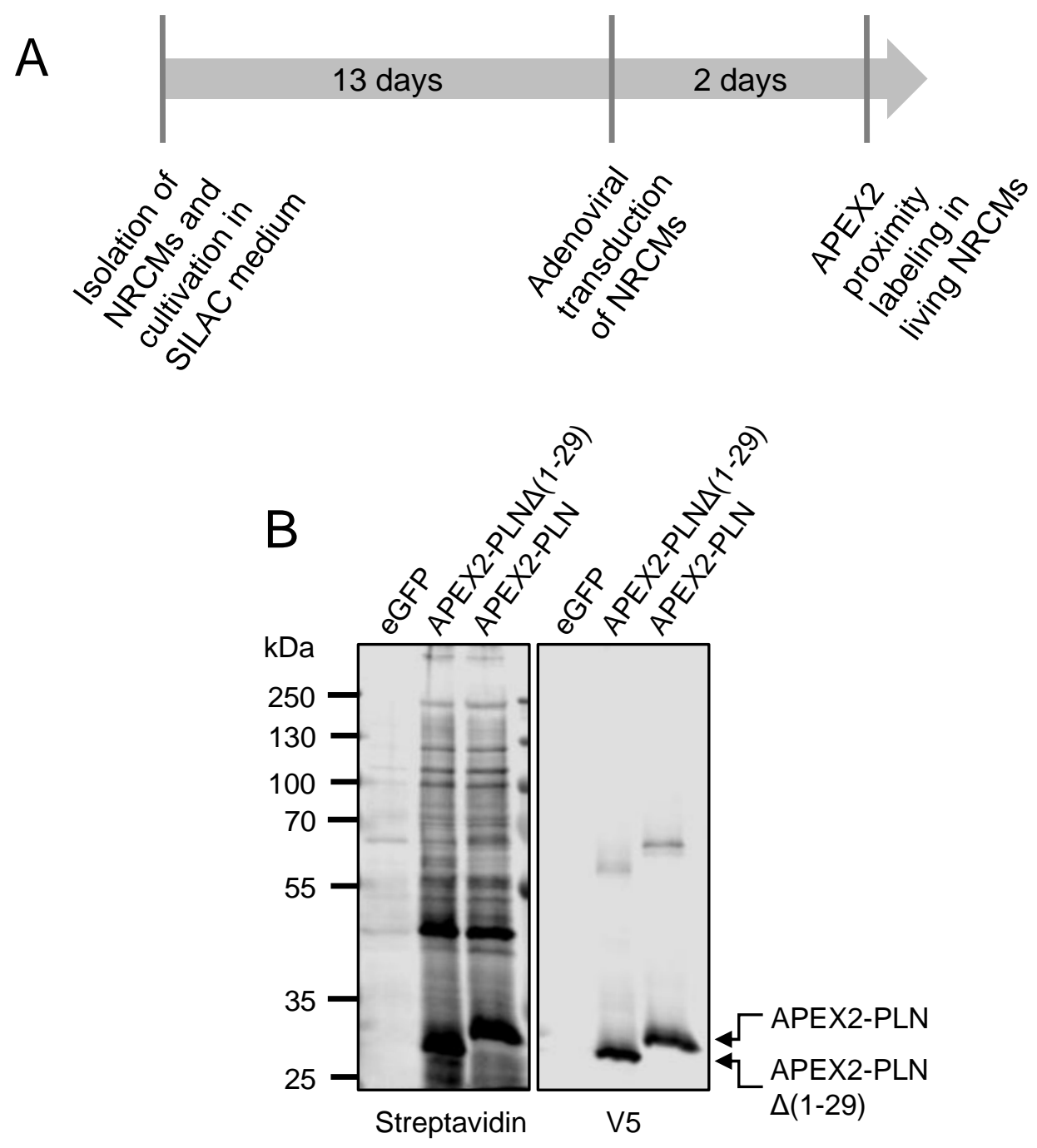

Figure 32 PLN targeted APEX2 proximity labeling in NRCMs for ratiometric proteomics

A, Experimental time scale for ratiometric APEX2 proximity labeling in APEX2-PLN versus APEX2PLNA(1-29) transfected NRCMs. B, Immunoblot analysis showed APEX2 dependent biotinylation of proteins upon biotin-phenol and $\mathrm{H}_{2} \mathrm{O}_{2}$ treatment. $\mathrm{V} 5$ antibody from the same blot showed equal expression of APEX2-PLN and APEX2-PLN $\triangle(1-29)$ proteins. $n=5$.

For ratiometric proteomics, a three-state SILAC experiment was designed. To exclude effects by the labeling medium, a systematic label switch was performed (Figure $33 \mathrm{~A}$ ). In total, five 
experiments were performed of which three experiments were performed in parallel (technical replicates; labeling I, II, and III), followed by two independent experiments (biological replicates, labeling II and III). After the proximity labeling in transduced NRCMs, lysates were tested by mass spectrometry for the incorporation of labeled amino acids resulting in a minimum of $96.5 \%$ incorporation. Next, lysates from each experiment were mixed in a ratio of $1: 1: 1$, and biotinylated proteins were enriched by avidin affinity purification (Figure 33C). The elution fraction of all five experiments was analyzed by ratiometric mass spectrometry (LCMS/MS).

Taken together, isolated NRCMs were successfully radiolabeled and transduced by adenoviruses for five experiments. PLN specific APEX2 proximity labeling was performed in living cells, and afterward, biotinylated proteins were enriched for ratiometric mass spectrometry analysis. 
A

$\overbrace{\text { I }}^{\text {Three-state SILAC experiment }}$

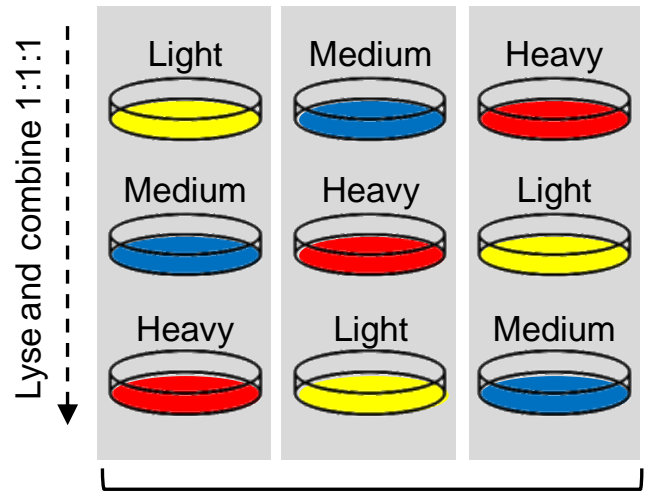

Avidin beads

LC-MS/MS
eGFP

APEX2-PLN $\Delta(1-29)$

APEX2-PLN

B

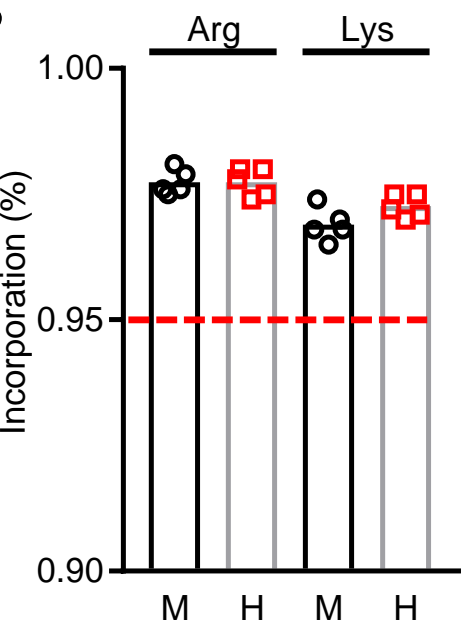

C

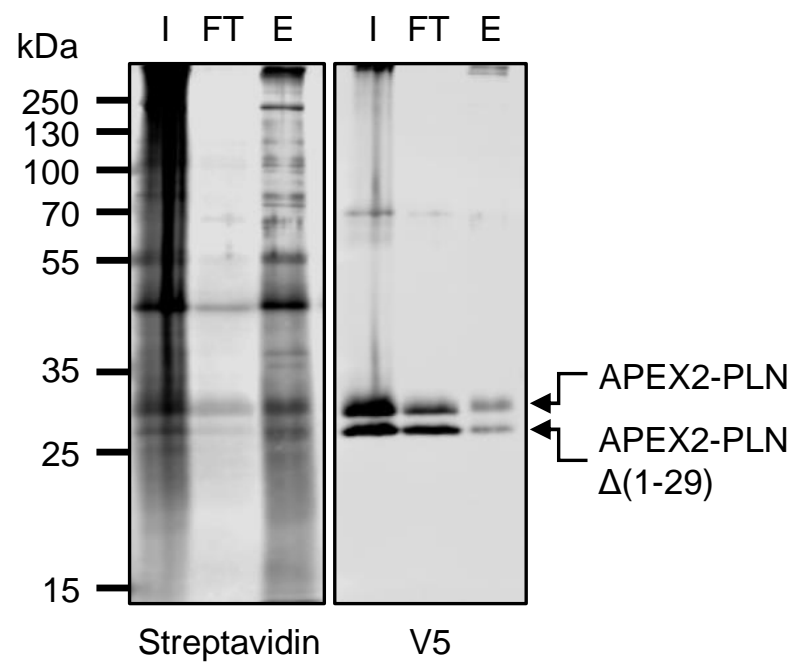

Figure 33 Ratiometric SILAC APEX2 proximity proteomics in NRCMs

A, Workflow for quantitative protein radiolabeling in NRCMs by three-state SILAC experiments with systematic label switch. Light: Arg-0 and Lys-0; Medium: Arg-6 and Lys-4 ; Heavy: Arg-10 and Lys-8. Adenoviral transduction of NRCMs with APEX2-PLN, APEX2-PLNA(1-29), and eGFP was followed by APEX2 proximity labeling and cell lysis. After mixing lysates in a ratio of 1:1:1, biotinylated proteins were enriched using avidin beads and analyzed by mass spectrometry (LC-MS/MS). $n=5$ (technical replicates: I, II, III; biological replicates with the same labeling scheme: II, III). B, Mass spectrometry (LC-MS/MS) quantified $>96.5 \%$ L-arginine (Arg) and L-lysine (Lys) incorporation (red line, 95\%). Of note, the scale starts at 0.90 . C, Lysates of radiolabeled and adenoviral transduced NRCMs were mixed in a ratio of 1:1:1 after induced biotinylation. Immunoblot showed enrichment of biotinylated proteins after affinity purification. The elution fractions of all five experiments were analysed by ratiometric mass spectrometry. I, input; FT, flow through; E, eluate. 


\subsubsection{Analysis of ratiometric PLN targeted APEX2 proteomic data}

Mass spectrometry data were normalized, and ratios of the individually labeled experiments were calculated by MaxQuant software. Logarithmic base $2\left(\log _{2}\right)$ was used as a ratiometric value. Resulted ratios of $\geq 0(\geq 1: 1)$ were APEX2-PLN enriched over the controls APEX2PLN $\Delta$ (1-29) or eGFP. For data analysis, only proteins detected in all five experiments were used (765 proteins). To demonstrate the enrichment of biotinylated proteins for APEX2-PLN, histograms were plotted for $\log _{2}$ ratios of APEX2-PLN / eGFP and APEX2-PLN $\Delta(1-29)$ versus the number of proteins showing a right shifted (ratios $\geq 0$ ) distribution in all five experiments (Figure 34). 


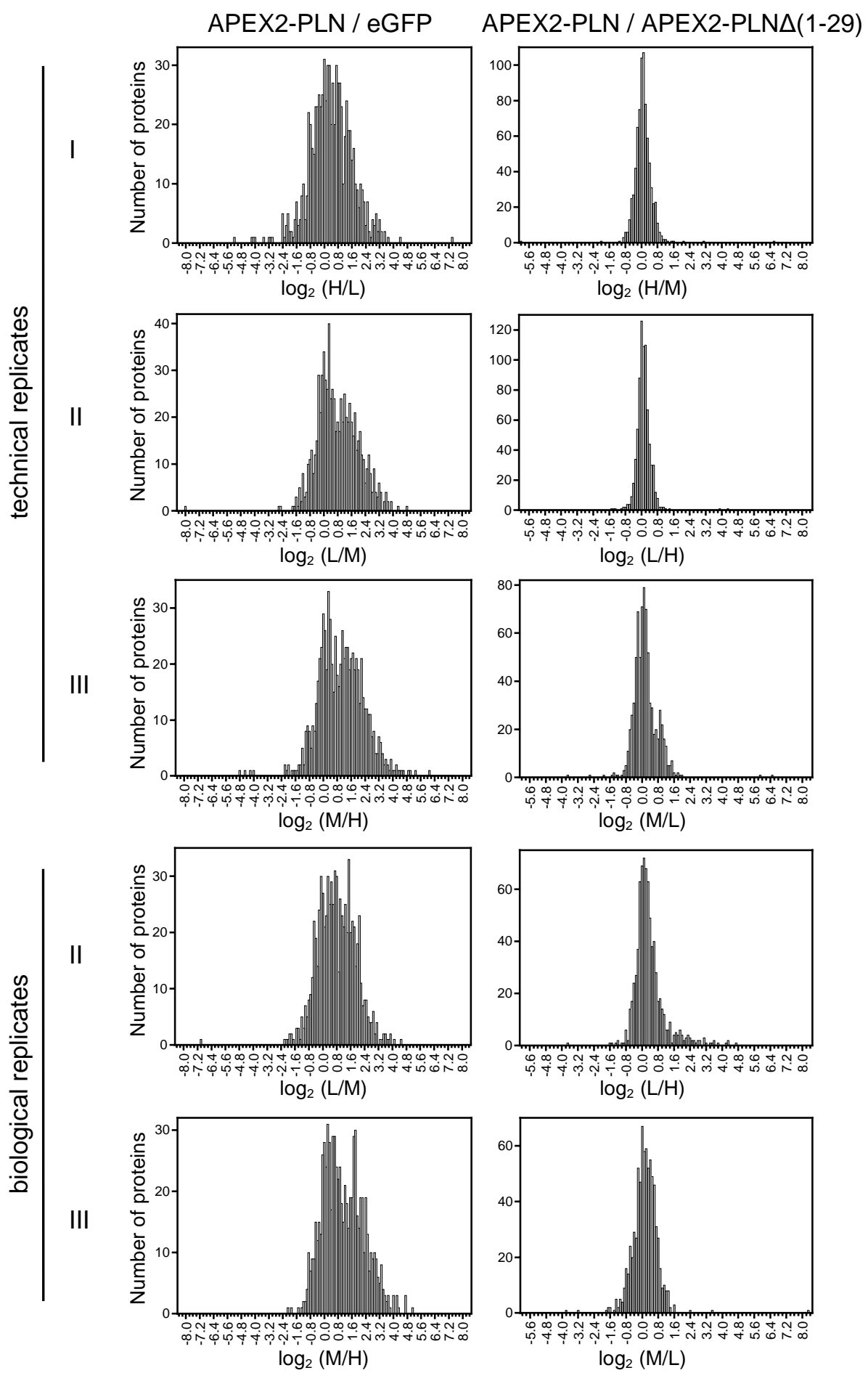

Figure 34 Analysis of ratiometric PLN targeted APEX2 proteomic data

Histograms of normalized $\log _{2}$ ratios APEX2-PLN / eGFP and APEX2-PLN / APEX2-PLN $\Delta(1-29)$ plotted against the number of proteins for all experimental replicates with systematic label switch $(H$, heavy; $M$, medium; L, light). Histograms show broad right-shifted distribution for $\log _{2}$ ratios $>0$. Proteins identified in all five experiments (technical and biological replicates) were analyzed (765 proteins). Bin size $=0.1$. $n=5$. 
Furthermore, the $\log _{2}$ ratios of 765 detected proteins were plotted against their total intensity (Figure 35A,B). PLN was detected on the right end of the distribution (Figure 35A) or isolated from the cloud as the most enriched protein (Figure 35B). SERCA2a, a direct PLN interactor, was detected as one of the most abundant proteins but showed no significant enrichment $\left(\log _{2}\right.$ $<0)$. To assess which proteins were significantly enriched for APEX2-PLN, a statistical z-test was performed, testing against 0 , and resulted in 133 significantly enriched proteins over APEX2-PLN $\triangle(1-29)$ and eGFP (Figure 35C). Phospholamban was significantly detected in both ratios with the highest $\log _{2}$ value. Moreover, PPP1cb and PPP2r1a were identified as known PLN as interacting proteins ${ }^{38,125}$. 
A

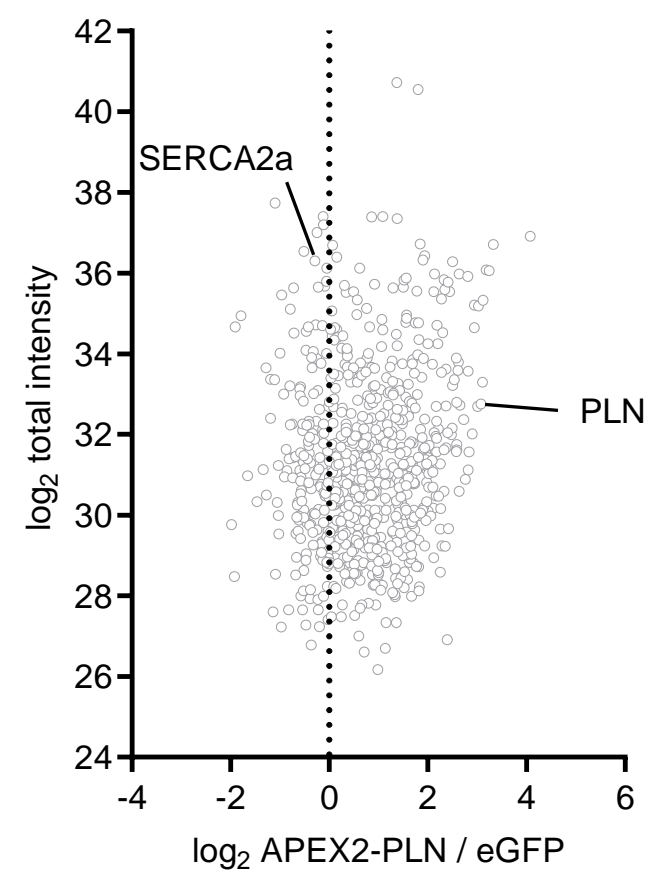

$\mathrm{B}$

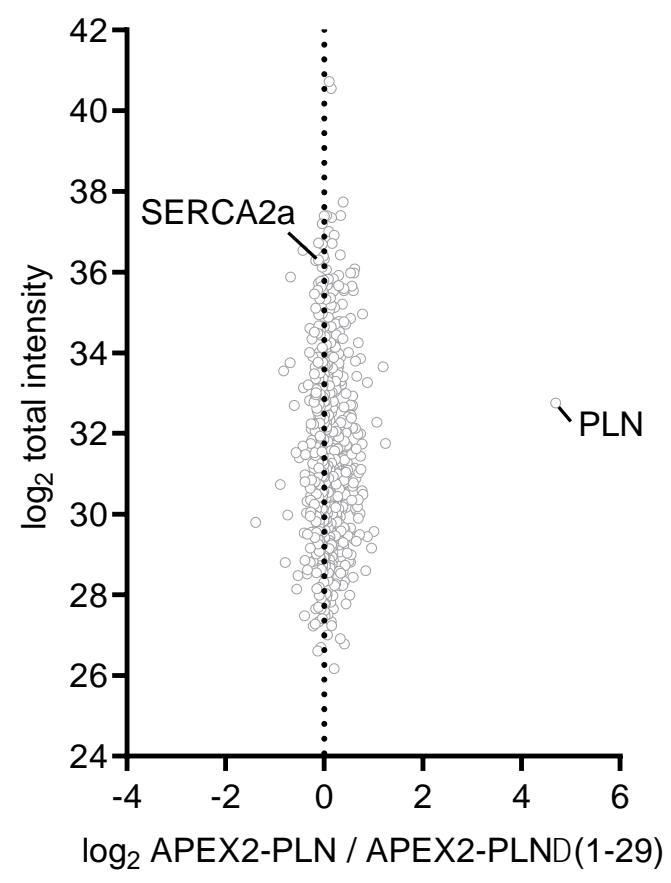

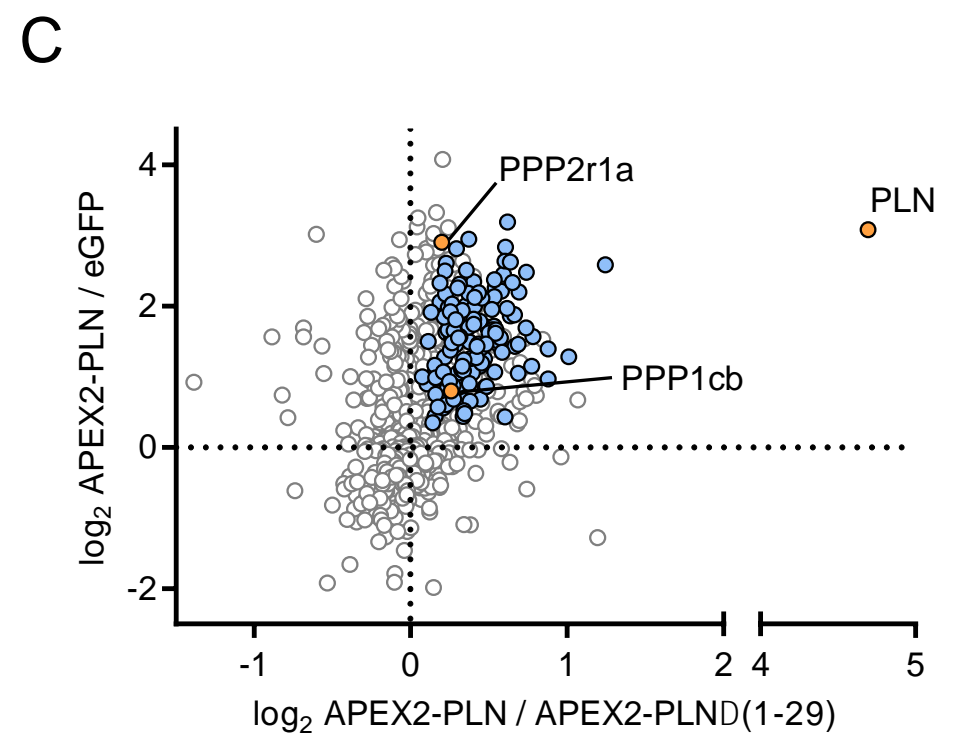

Figure 35 Protein enrichment after APEX2-PLN proximity labeling in NRCMs

Average $\log _{2}$ ratios of APEX2-PLN vs. eGFP (A) or APEX2-PLN $\Delta(1-29)$ (B) plotted against $\log _{2}$ of total intensities and show a right-shifted distribution ( $\log 2>0)$. SERCA2a has been detected as one of the most abundant proteins in all samples. C, Log 2 ratios of APEX2-PLN vs. APEX2-PLN $\Delta(1-29)$ or eGFP show 765 identified proteins (white points) of which 133 has been significantly enriched for APEX2-PLN (blue and orange points). PLN was one of the most enriched proteins. The cytosolic proteins PPP2r1a and PPP1cb are known interaction partners of PLN and were significantly enriched by APEX2-PLN. 
Gene enrichment analysis of the 133 significantly enriched proteins using the online ToppGene Suite tool ToppFun showed significant enrichment of the 14-3-3 protein family (Figure 36A). The top six enriched gene families were listed and visualized as data points plotted for $\log _{2}$ ratios of APEX2-PLN / APEX2-PLN 4 (1-29) and APEX2-PLN / eGFP (Figure 36B). 
14-3-3 proteins ID 1053

Peroxiredoxins ID 953

LIM domain containing ID 1218

Septins ID 732

Troponin complex subunits ID 1219

Small heat shock proteins ID 585

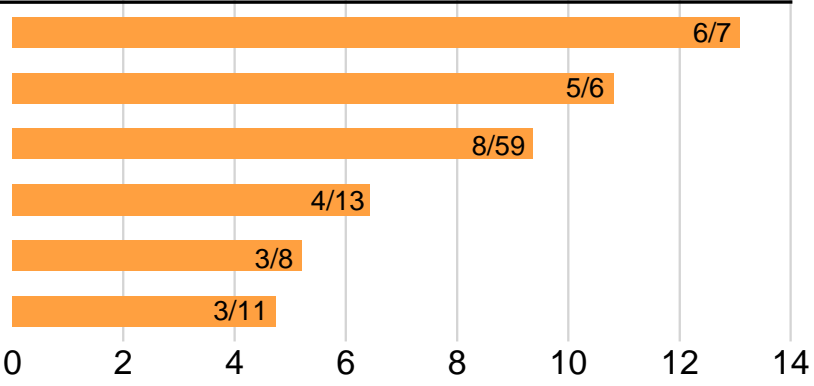

B
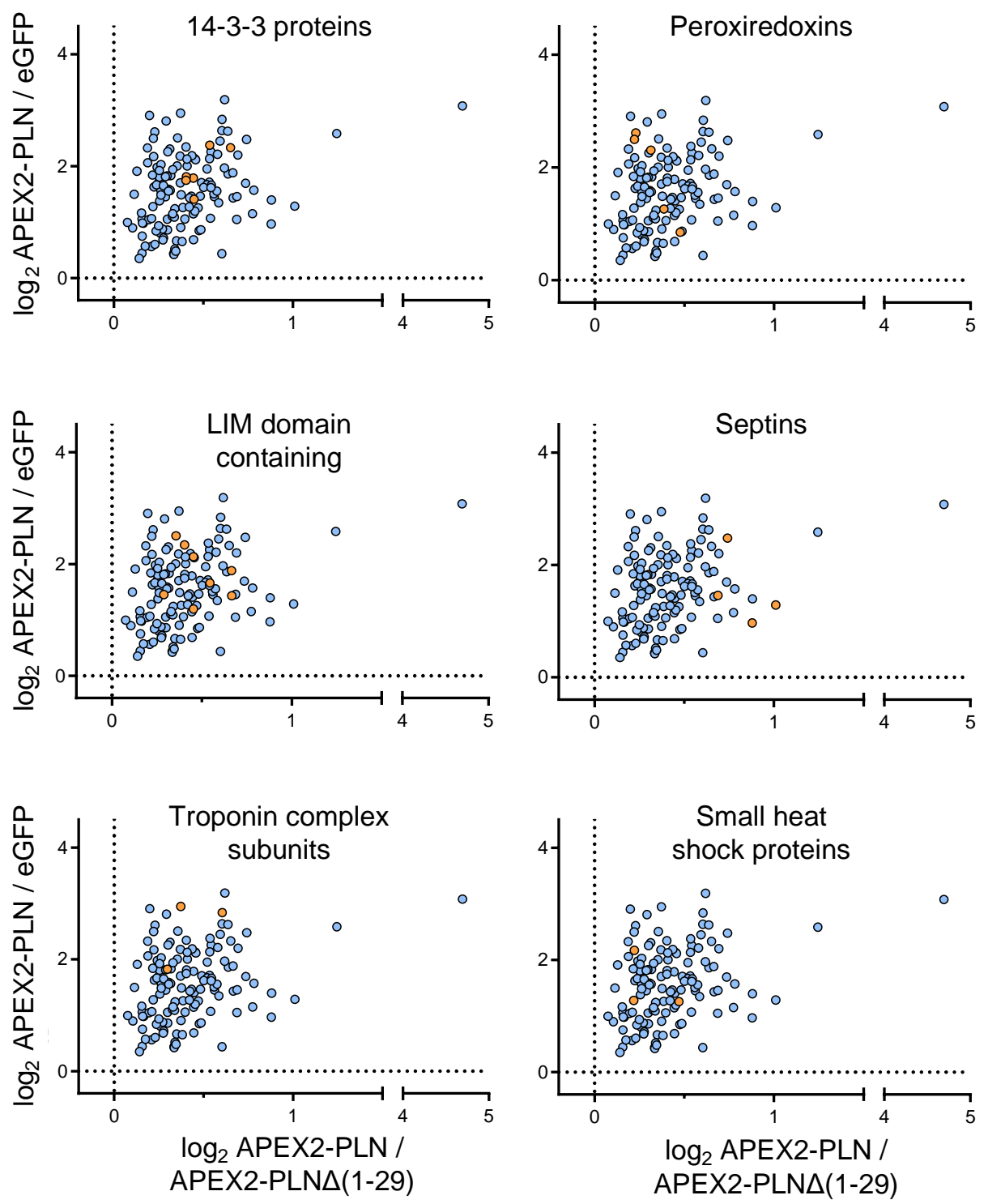

Figure 36 Gene enrichment analysis of APEX2-PLN enriched proteins

A, APEX2-PLN specific proteins were enriched for their biological functions using ToppGene Suite tool ToppFun (default setting: FDR correction, p-value cut off at 0.05 and gene limit set between and including 1 and 2000 per pathway). Bar graphs show the top six enriched gene families based on APEX2-PLN enriched proteins. Numbers in bar graphs indicate the detected proteins of the group. B, 
APEX2-PLN significantly enriched proteins were plotted for $\log _{2}$ ratios of APEX2-PLN / APEX2-PLN $\triangle$ (129) and APEX2-PLN / eGFP. Orange points show the gene family enriched proteins from A. Blue points show all significantly enriched proteins. For detailed protein list see Apendix (Table 9,Table 10). 


\subsection{Discussion}

The exploration of novel PLN interaction partners is of high importance to understand how it is molecularly regulated and to develop rationales for new therapeutic interventions for patients and animals with heart disease.

This study was designed to identify potential new protein interactions of PLN. A proximitybased labeling technique for living primary heart cells was developed and combined with a quantitative proteomic analysis strategy. For live-cell labeling, the engineered ascorbate peroxidase APEX2 was fused N-terminally to PLN. After establishing the expression, localization, and functionality in HEK293A cells, neonatal rat cardiomyocytes were used as the primary model system. The advantages of this NRCM based proteomic labeling system are culture conditions that provide week-long observation periods and spontaneous beating and maturation of the cell population ${ }^{120}$. Additionally, NRCMs can be targeted by DNA vectors for genetic modifications as described previously by others ${ }^{120,126,127}$. Furthermore, a high degree of incorporation of stable isotope-labeled amino acids in cell culture (SILAC) was established for NRCMs. Hence, a ratiometric proteomic analysis was used after PLN targeted APEX2 proximity labeling in living cells. Targeting the cells with adenoviral vectors was used for efficient transduction. The ratiometric APEX2 proximity labeling in NRCMs identified proteins in nanometric proximity to PLN. In particular, 14-3-3 protein isoforms were identified, which function as phosphoadaptor proteins. It was predicted before that PLN contains a target motif for $14-3-3^{128}$. Hence, we hypothesized that 14-3-3 binding protects phospholamban from dephosphorylation, which may prolong the Ca uptake activity by SERCA2a after ß-adrenergic stimulation. This interaction was established in collaboration and is now part of a manuscript in preparation by Julia Menzel et al. (Prof. Dr. Blanche Schwappach, Department of Molecular Biology, University Medical Center, Göttingen).

\subsubsection{PLN targeted APEX2 proximity labeling in HEK293A and NRCM}

It has been shown before that PLN can N-terminally be tagged with a biosensor and is localized to the SERCA2a compartment ${ }^{129}$. Based on the same principle, PLN was N-terminally tagged with the ascorbate peroxidase APEX2 for the identification of novel interaction partners. APEX2 proximity labeling was previously established in several studies to map the mitochondrial intermembrane space ${ }^{68}$, cilia $^{70}$, or the spatiotemporal proximity of G-protein coupled receptors ${ }^{71}$. Whereas BiolD has a biotinylation radius of $10 \mathrm{~nm}^{115}$, APEX2 has a proximate radius of $<20 \mathrm{~nm}^{118}$ and is described as a reactive cloud with a diffusion gradient. Therefore, APEX2 is not only labeling direct interaction partners but additionally proximal proteins. APEX2 has a high enzymatic activity and allows for a short labeling time, i.e. a 
snapshot within $1 \mathrm{~min}$, whereas the labeling with BiolD is designed to as a cumulative approach to identify interactors over hours ${ }^{66}$. However, the treatment of cells with hydrogen peroxide, to initiate the biotinylation reaction by APEX, can cause oxidative stress ${ }^{130}$ or altered protein interactions ${ }^{131}$.

\subsubsection{APEX2-PLN targeting in neonatal rat cardiomyocytes}

After the APEX2-PLN localization and peroxidase function were successfully established in HEK293A cells, NRCMs were used as a primary cardiac cell model. Adult cardiomyocytes show typical alterations in morphology under culturing conditions (within hours) and are generally reported to be difficult to transfect with non-viral approaches ${ }^{120}$. The transfection efficiency in NRCMs using the calcium-phosphate co-precipitation method, however, is also low $(1-2 \%)^{126}$. Low transfection efficiency was confirmed for NRCM transfection by confocal imaging (data not shown). Therefore, adenoviral vectors were used for efficient gene transfer ${ }^{127}$. In order to achieve abundant expression of APEX2-PLN and APEX2-PLN $\Delta$ (1-29) in NRCMs, recombinant adenoviral vectors (serotype V) were generated and amplified (Sirion Biotech, Martinsried).

\subsubsection{Quantitative proteomic analysis of NRCMs}

Quantitative proteomic approaches are essential to determine global proteomic changes in cells, tissue, or organisms. As Mass spectrometry is not quantitative per se, stable isotope labeling by amino acids in cell culture (SILAC) is one relative quantitation method ${ }^{123}$. SILAC uses the incorporation of isotope-labeled amino acids into proteins during cell culturing. Therefore, the culture medium is lacking an essential amino acid, which is supplemented with the isotope-labeled form instead. Ong and Mann reported complete incorporation in NIH 3T3 fibroblast after five cell divisions ${ }^{123}$. Feger et al. used SILAC in NRCMs and reached a relative isotope abundance of $\sim 40 \%$ after $120 \mathrm{~h}^{124}$. However, no additional data became available about the SILAC incorporation in non-proliferating primary NRCMs. Thus, it was systematically tested for up to 20 days. That resulted in isotope-labeled amino acid incorporation of over $>95 \%$ after 13 days (Figure 29). Finally, NRCMs were established as a suitable cell model for APEX2 proximity labeling combined with quantitative proteomics.

\subsubsection{Analysis of ratiometric PLN targeted APEX2 proteomic data}

The calculated $\log _{2}$ ratio of APEX2-PLN enriched proteins against APEX2-PLN $\Delta(1-29)$ or eGFP showed a right-shifted distribution, in particular, values $\log _{2} \geq 0$. As expected, the right 
shift of both ratios differs and shows for eGFP control a higher spectrum compared to the SRtargeted control APEX2-PLN $\Delta$ (1-29). eGFP was used as transduction control and to exclude unspecific enrichment of protein interactions due to affinity purification. APEX2-PLN $\Delta(1-29)$ was used to exclude SR resident proteins as false-positive enriched hits. Additionally, overlapping interactors for both PLN and PLN $\Delta$ (1-29) are expected to be biotinylated to the same degree and show no enrichment $\left(\log _{2}\right.$ ratio $\left.<0\right)$. Therefore, the $\log _{2}$ ratio variance of APEX2-PLN over APEX2-PLN $\Delta$ (1-29) was smaller compared to the $\log _{2}$ ratio variance over eGFP. In order to cover all PLN interactions, an additional soluble cytosolic APEX control will be applied. Against expectation, SERCA2a, a direct PLN interactor, has been detected but was not significantly enriched. That is, while the total abundance for each $\log _{2}$ ratio revealed SERCA2a as one of the most abundant proteins in the samples. Here suggests that biotinylated SERCA2a proteins get lost in the background signals.

Nonetheless, previously established interactions were significantly enriched for PLN: PPP1cb and PP2r1a. The protein phosphatase 1 catalytic subunit $\beta / \delta$ (PPP1cb) is one of four isoforms ${ }^{132}$ and active as a holoenzyme together with one or more regulatory subunits. PP1 accounts for $70 \%$ of PLN phosphatase activity towards the PKA phosphorylation site Ser-16 and CamK2 phosphorylation site Thr- $17^{38}$. Additionally, the protein phosphatase $2 \mathrm{~A}$ scaffolding subunit Aa (PPP2r1a) is one of two constant regulatory subunit isoforms of PP2. Together with the catalytic subunit, they form the core enzyme complex ${ }^{133}$. Moreover, to build the PP2A holoenzyme, the core enzyme binds to one out of nine different regulatory subunits, which facilitates the targeting to distinct and well defined subcellular localizations ${ }^{133}$. Compared to PP1, the PP2A complex accounts for the remaining $30 \%$ of PLN dephosphorylation ${ }^{125}$. Taken together, two phosphatase subunits were correctly labeled in the proximity of PLN in living NRCMs, proving the ability of this PLN-based proximity assay to label transient protein interactions.

\subsubsection{Gene enrichment analysis of APEX2-PLN enriched proteins}

Gene enrichment analysis of 133 significantly enriched proteins revealed several gene families. The enriched gene family with the highest $p$-value was the 14-3-3 protein. 14-3-3 proteins are phosphoadaptor proteins, of which several isoforms are expressed in the heart. Here, six out of seven isoforms were enriched for APEX2-PLN. They bind to proteins that contain phosphorylated Ser/Thr residues within specific sequence motifs ${ }^{134,135}$. Interestingly, an Arg-based PLN motif has been predicted as the mechanism of the 14-3-3 protein interaction $^{128}$. The peptide-binding groove of heterodimeric 14-3-3 proteins is highly conserved, and all 14-3-3 proteins bind to similar peptide motifs ${ }^{135,136}$, suggesting that all isoforms can bind to PLN. While both PLN and SLN function as inhibitory regulators of 
SERCA2a in the heart, only PLN is predicted as 14-3-3 target, when Ser-16 or Thr-17 is phosphorylated. In order to look at other SERCA2a regulating micropeptides ${ }^{26}$, another-regulin (ALN) was predicted to be a 14-3-3 target when Ser-19 is phosphorylated, together with myoregulin (MLN), when Thr-12 is phosphorylated ${ }^{137}$. That suggests that $14-3-3$ interactions with SERCA regulating micropeptides are a general mechanism not restricted to the heart. An important consequence of 14-3-3 binding to PLN will be a protection from dephosphorylation by steric masking, prolonging the increased SERCA2a activity during B-adrenergic stimulation. Next, five out of six peroxiredoxins (Prdx) were significantly enriched through APEX2-PLN labeling. Prdxs represent a diverse family of antioxidant and redox signaling proteins and are highly expressed in the heart ${ }^{138}$. They may confer protection against oxidative stress and are well known to inhibit apoptosis mediated by hydrogen peroxide ${ }^{139} . \mathrm{H}_{2} \mathrm{O}_{2}$ is a substrate of $\operatorname{Prdxs}$ that catalyze its reduction ${ }^{138}$. This reaction is also needed for the oxidation of Prdxs to form heterodimers through inter- and intramolecular disulfide bonds ${ }^{138}$. In the context of the APEX2 proximity labeling initiated with $1 \mathrm{mM} \mathrm{H}_{2} \mathrm{O}_{2}$, Prdxs activity may protect other proteins from $\mathrm{H}_{2} \mathrm{O}_{2}$. Interestingly, Prdxs were enriched over the APEX2-PLN $\Delta(1-29)$ control. That suggests a specific enrichment of Prdx proteins for APEX2-PLN. It has been reported that Prdx oxidation was associated with translocation from the cytosol to the membrane and myofilament-enriched fractions ${ }^{138}$. This mechanism may explain the proximity to PLN and the resulting labeling of Prdxs upon oxidation. Interestingly, Prdx-II protein expression is increased in PLN knockout hearts ${ }^{140}$, and it is hypothesized that it may serve as a cardioprotective mechanism ${ }^{141}$. Additionally, cardiac-specific overexpression of Prdx-II leads to reduced PLN Ser-16 phosphorylation, while total PLN protein levels were unchanged ${ }^{142}$. Hence, it was speculated that Prdx-II may regulate cardiac contractility through altered PLN phosphorylation levels ${ }^{142}$ or may play a role for cardiomyocyte apoptosis ${ }^{141}$.

Along with 14-3-3 proteins and peroxiredoxins, the group of LIM domain containing proteins was enriched for APEX2-PLN. The LIM domain is a specialized double-zinc finger motif defining a large protein family. Some are mainly expressed in mammalian hearts playing a pivotal role in organizing the cytoskeleton, signal transduction, gene expression, cardiomyocyte metabolism, and cell differentiation ${ }^{143}$. LIM domain proteins are thought to function as a protein-protein interaction module, facilitating contacts between proteins of functional complexes ${ }^{144}$. Eight LIM domain containing proteins were enriched by APEX2-PLN. Considering established functions of LIM domain containing proteins, they seem to be proximal rather than directly bound to PLN.

Another group enriched for APEX-PLN is the protein family of septins (SEPT). Septins are GTP binding proteins involved in cytokinesis and other biological processes, including cell polarity, exocytosis, ciliogenesis, phagocytosis, motility, and apoptosis ${ }^{145}$. Septins can assemble into a filamentous protein complex in the cell. They can directly interact with 
membranes, microfilaments, and microtubles ${ }^{145}$. In the developing heart, a significant level of expression has been found for SEPT2, SEPT6, SEPT7, and SEPT9, whereas no detectable expression was found in the adult heart ${ }^{146}$. Interestingly, the PLN interaction partner HAX$1^{45,147}$ was reported to modulate changes in septin signalling ${ }^{148}$. Due to the loss of SEPT expression in the adult heart, there seems to be no physiological role for interactions with PLN in adult cardiomyocytes.

Furthermore, the group of troponin complex subunits was significantly enriched for APEX2PLN. The cardiac troponin consists of three subunits ${ }^{149,150}$, which are troponin C (TnC), troponin I (Tnl), and troponin T (TnT). All three subunits were labeled in the proximity of PLN. $\mathrm{TnC}$ is the $\mathrm{Ca}^{2+}$-binding subunit that inhibits the actomyosin interaction, $\mathrm{Tnl}$ is an inhibitory component of contractile interaction, and $\mathrm{TnT}$ is a tropomyosin-binding component. All three subunits are involved together with myosin, actin, and tropomyosin in the $\mathrm{Ca}^{2+}$-regulated physiological muscle contraction ${ }^{151}$. Interestingly, both $\mathrm{PLN}^{92}$ and $\mathrm{TnI}^{152}$ are targets of PKA in ventricular myocytes and are phosphorylated upon B-adrenergic stimulation. Tnl phosphorylation increases the $\mathrm{Ca}^{2+}$ dissociation from $\mathrm{TnC}^{153}$ and accelerates muscle relaxation, similar to PLN phosphorylation ${ }^{27}$. Taken together, it appears that troponin and PLN are in close proximity rather than direct interactors by sharing the same protein kinase.

Finally, the family of small heat shock proteins was significantly enriched. Small heat shock proteins function as molecular chaperones supporting the assembly, disassembly, stabilization, and transport of intracellular proteins ${ }^{154}$. The small heat shock protein 20 (HSPB6) is known to regulate PLN dephosphorylation ${ }^{43}$. HSPB6 inhibits the enzymatic activity of PP1, which was associated with an increased level of PLN phosphorylation and $\mathrm{SR} \mathrm{Ca}^{2+}$ transport ${ }^{43}$. This finding confirms again the specificity of the PLN targeted APEX2 proximity assay, detecting also secondary interactions.

In summary, a PLN targeted APEX2 proximity labeling assay was established in NRCMs to identify novel candidate interaction partners as a primary hypothesis-generating screen. Neonatal cardiomyocytes were cultivated in SILAC medium as a ratiometric proteomic approach. Additionally, a protocol for adenoviral NRCM transduction was established. Based on three technical and two biological replicates, 133 proteins were significantly enriched following APEX2-PLN labeling in living NRCMs. Among these hits, several protein families have been identified with 14-3-3 proteins as the most significant interaction candidates. Overall, APEX2 proximity labeling was used as a screening tool and combined with additional methods to confirm and study protein-protein interactions. Furthermore, it will be of high interest, which physiological consequences PLN mutations like R14del or R9C will have on the newly identified protein interactions in human cardiomyopathy. 


\section{Conclusion and Outlook}

This study aimed to identify previously unknown PLN protein interactions by using two different unbiased proteomic approaches. First, the complexome profiling analysis of isolated ventricular cardiomyocyte membranes was established and showed known and previously unknown multi-protein complexes. Mitochondrial OXPHOS complexes were used for quality control, and their assembly into supercomplexes could be observed. The Na,K-ATPase was captured in two different states, with and without its regulator phospholemman. Additionally, we could show that PLN and SERCA2a are highly associated and are associated with macromolecular complexes. Importantly, at higher molecular weight, a novel SR $\mathrm{Ca}^{2+}$ cycling complex comprised of the RyR2 calcium release channel, SERCA2a, and each regulatory protein subunits, was identified. This finding was part of the published manuscript by Alsina et al., showing that the regulatory subunit PPP1R3A targets PP1 not only to PLN but also to the RyR2 channels and is part of the SR $\mathrm{Ca}^{2+}$ cycling complex. Interestingly, this complex was altered in PLN-deficient mice showing the abundance of PLN as an important mediator.

Furthermore, hierarchical cluster analysis revealed SR localized SLMAP as a potential interactor of PLN. It was shown that SLMAP is localized adjacent to PLN in mouse VCM by STED microscopy and could be co-eluted with PLN by affinity purification. Remarkably, as proteins involved in $\mathrm{Ca}^{2+}$ handling are downregulated during the progression of heart failure, SLMAP showed increased protein expression in mice four weeks after TAC surgery. Also, PLN-deficient mice showed higher abundance in high molecular weight complexes compared to WT and increased protein expression of SLMAP. Also, during differentiation and maturation of human iPSC towards a cardiac phenotype, SLMAP protein expression increases. This suggests an important role of SLMAP during heart differentiation and remodeling.

Moreover, PLN targeted APEX2 proximity labeling was established in living NRCMs and gave further insights into the interactome of PLN. Gene enrichment analysis identified several proteins and gene families, which were specifically labeled in the proximity of PLN. Six of seven 14-3-3 proteins were significantly enriched in the proximity of PLN, together with peroxiredoxins, Lim domain containing proteins, septins, troponin complex subunits, and small heat shock proteins. 
In summary, two different proteomic approaches revealed previously unknown PLN protein interactions, providing new insights into the interactome of PLN. The identified interactors will be subject to future studies to elucidate their functional role in health and disease. This will provide the development of rationales for new therapeutic interventions for patients and animals with heart disease. 


\section{References}

1. Bers, D. M. Calcium Cycling and Signaling in Cardiac Myocytes. Annu. Rev. Physiol. 70, 23-49 (2008).

2. Fearnley, C. J., Llewelyn Roderick, H. \& Bootman, M. D. Calcium signaling in cardiac myocytes. Cold Spring Harb. Perspect. Biol. 3, (2011).

3. MacLennan, D. H. \& Kranias, E. G. Phospholamban: a crucial regulator of cardiac contractility. Nat. Rev. Mol. Cell Biol. 4, 566-577 (2003).

4. Periasamy, M. \& Kalyanasundaram, A. SERCA pump isoforms: Their role in calcium transport and disease. Muscle Nerve 35, 430-442 (2007).

5. Gianni, D., Chan, J., Gwathmey, J. K., Del Monte, F. \& Hajjar, R. J. SERCA2a in heart failure: Role and therapeutic prospects. Journal of Bioenergetics and Biomembranes vol. 37 375-380 (2005).

6. Martonosi, A. N. \& Pikula, S. The structure of the Ca2+-ATPase of sarcoplasmic reticulum. Acta Biochim. Pol. 50, 337-365 (2003).

7. Gélébart, P., Martin, V., Enouf, J. \& Papp, B. Identification of a new SERCA2 splice variant regulated during monocytic differentiation. Biochem. Biophys. Res. Commun. 303, 676-684 (2003).

8. Dally, S. et al. Ca2+-ATPases in non-failing and failing heart: Evidence for a novel cardiac sarco/endoplasmic reticulum Ca2+-ATPase 2 isoform (SERCA2c). Biochem. J. 395, 249-258 (2006).

9. Dode, L. et al. CDNA cloning, expression and chromosomal localization of the human sarco/endoplasmic reticulum Ca2+ -ATPase 3 gene. Biochem. J. 318, 689-699 (1996).

10. Bobe, R. et al. Identification, expression, function, and localization of a novel (sixth) isoform of the human sarco/endoplasmic reticulum Ca2+ATPase 3 gene. J. Biol. Chem. 279, 24297-24306 (2004).

11. Bublitz, M. et al. Ion pathways in the sarcoplasmic reticulum Ca2+-ATPase. Journal of Biological Chemistry vol. 288 10759-10765 (2013).

12. Toyoshima, C. et al. Crystal structures of the calcium pump and sarcolipin in the Mg 2+bound E1 state. Nature 495, 260-264 (2013).

13. Winther, A. M. L. et al. The sarcolipin-bound calcium pump stabilizes calcium sites exposed to the cytoplasm. Nature 495, 265-269 (2013).

14. Sitsel, A. et al. Structures of the heart specific SERCA2a Ca2+ - ATPase. EMBO J. 38, (2019).

15. Wuytack, F., Raeymaekers, L. \& Missiaen, L. Molecular physiology of the SERCA and SPCA pumps. Cell Calcium (2002) doi:10.1016/S0143416002001847.

16. Toyoshima, C., Nakasako, M., Nomura, H. \& Ogawa, H. Crystal structure of the calcium 
pump of sarcoplasmic reticulum at $2.6 \AA$ resolution. Nature 405, 647-655 (2000).

17. Kühlbrandt, W. Biology, structure and mechanism of P-type ATPases. Nature Reviews Molecular Cell Biology vol. 5 282-295 (2004).

18. Zhihao, L. et al. SERCA2a: a key protein in the Ca2+ cycle of the heart failure. Heart Failure Reviews (2019) doi:10.1007/s10741-019-09873-3.

19. Kranias, E. G. \& Hajjar, R. J. Modulation of cardiac contractility by the phopholamban/SERCA2a regulatome. Circ. Res. 110, 1646-1660 (2012).

20. $\mathrm{He}, \mathrm{H}$. et al. Overexpression of the rat sarcoplasmic reticulum $\mathrm{Ca} 2+\mathrm{ATPase}$ gene in the heart of transgenic mice accelerates calcium transients and cardiac relaxation. $J$. Clin. Invest. 100, 380-389 (1997).

21. Baker, D. L. et al. Targeted overexpression of the sarcoplasmic reticulum Ca2+-ATPase increases cardiac contractility in transgenic mouse hearts. Circ. Res. 83, 1205-1214 (1998).

22. Vetter, R. et al. Transgenic overexpression of the sarcoplasmic reticulum Ca2+ATPase improves reticular Ca2+ handling in normal and diabetic rat hearts. FASEB J.16, 16571659 (2002).

23. Periasamy, M. et al. Impaired cardiac performance in heterozygous mice with a null mutation in the sarco(endo)plasmic reticulum Ca2+-ATPase isoform 2 (SERCA2) gene. J. Biol. Chem. 274, 2556-2562 (1999).

24. Schultz, J. E. J. et al. Accelerated onset of heart failure in mice during pressure overload with chronically decreased SERCA2 calcium pump activity. Am. J. Physiol. Circ. Physiol. 286, H1146-H1153 (2004).

25. Ji, Y. et al. Disruption of a single copy of the SERCA2 gene results in altered Ca2+ homeostasis and cardiomyocyte function. J. Biol. Chem. 275, 38073-38080 (2000).

26. Anderson, D. M. et al. Widespread control of calcium signaling by a family of SERCAinhibiting micropeptides. Sci. Signal. 9, ra119-ra119 (2016).

27. LaRaia, P. J. \& Morkin, E. Adenosine 3',5' monophosphate dependent membrane phosphorylation. A possible mechanism for the control of microsomal calcium transport in heart muscle. Circ. Res. 35, 298-306 (1974).

28. MICHIHIKO TADA, MADELEINE A. KIRCHBERGER, A. A. M. K. Phosphorylation of a 22,000-dalton component of the cardiac sarcoplasmic reticulum by adenosine 3':5'monophosphate-dependent protein kinase. J. Biol. Chem. Vol. 250, 2640-2647 (1975).

29. T Cantilina, Y Sagara, G. I. and L. R. J. Comparative studies of cardiac and skeletal sarcoplasmic reticulum ATPases. Effect of a phospholamban antibody on enzyme activation by Ca2+. J. Biol. Chem. 268, 17018-17025. (1993).

30. Simmerman, H. K. \& Jones, L. R. Phospholamban: protein structure, mechanism of action, and role in cardiac function. Physiol Rev. 78, 921-947 (1998). 
31. Wittmann, T., Lohse, M. J. \& Schmitt, J. P. Phospholamban pentamers attenuate PKAdependent phosphorylation of monomers. J. Mol. Cell. Cardiol. 80, 90-97 (2015).

32. J Fujii, A Zarain-Herzberg, H F Willard, M. T. and D. H. M. Structure of the rabbit phospholamban gene, cloning of the human cDNA, and assignment of the gene to human chromosome 6. J. Biol. Chem. 266, 11669-11675 (1991).

33. MacLennan, D. H., Toyofuku, T. \& Kimura, Y. Sites of regulatory interaction between calcium ATPases and phospholamban. in Alterations of Excitation-Contraction Coupling in the Failing Human Heart 17-24 (Steinkopff, 1998). doi:10.1007/978-3-642-486708_2.

34. Jones, A. D. W. and L. R. Phosphorylation-induced mobility shift in phospholamban in sodium dodecyl sulfate-polyacrylamide gels. Evidence for a protein structure consisting of multiple identical phosphorylatable subunits. J. Biol. Chem. 259, 1834-1841 (1984).

35. Luo, W. et al. Transgenic approaches to define the functional role of dual site phospholamban phosphorylation. J. Biol. Chem. 273, 4734-4739 (1998).

36. Luo, W. et al. Targeted ablation of the phospholamban gene is associated with markedly enhanced myocardial contractility and loss of beta-agonist stimulation. Circ. Res. 75, 401-409 (1994).

37. Dash, R. et al. Interactions between phospholamban and $\beta$-adrenergic drive may lead to cardiomyopathy and early mortality. Circulation 103, 889-896 (2001).

38. Steenaart, N. A. E., Ganim, J. R., Di Salvo, J. \& Kranias, E. G. The phospholamban phosphatase associated with cardiac sarcoplasmic reticulum is a type 1 enzyme. Arch. Biochem. Biophys. (1992) doi:10.1016/0003-9861(92)90359-5.

39. Neumann, J. et al. Increased expression of cardiac phosphatases in patients with endstage heart failure. J. Mol. Cell. Cardiol. 29, 265-272 (1997).

40. Carr, A. N. et al. Type 1 Phosphatase, a Negative Regulator of Cardiac Function. Mol. Cell. Biol. 22, 4124-4135 (2002).

41. Ceulemans, H. \& Bollen, M. Functional Diversity of Protein Phosphatase-1, a Cellular Economizer and Reset Button. Physiological Reviews vol. 84 1-39 (2004).

42. KIRCHHEFER, U. et al. Enhanced cardiac function in mice overexpressing protein phosphatase Inhibitor-2. Cardiovasc. Res. 68, 98-108 (2005).

43. Qian, J. et al. Small heat shock protein 20 interacts with protein phosphatase- 1 and enhances sarcoplasmic reticulum calcium cycling. Circ. Res. 108, 1429-38 (2011).

44. Ren, X.-P. et al. MicroRNA-320 is involved in the regulation of cardiac ischemia/reperfusion injury by targeting heat-shock protein 20. Circulation 119, 23572366 (2009).

45. Vafiadaki, E. et al. Phospholamban Interacts with HAX-1, a Mitochondrial Protein with Anti-apoptotic Function. J. Mol. Biol. 367, 65-79 (2007). 
46. Schmitt, J. P. et al. Dilated cardiomyopathy and heart failure caused by a mutation in phospholamban. Science (80-. ). 299, 1410-1413 (2003).

47. Haghighi, K. et al. The human phospholamban Arg14-deletion mutant localizes to plasma membrane and interacts with the Na/K-ATPase. J. Mol. Cell. Cardiol. 52, 773782 (2012).

48. LIMAS, C. J. et al. Calcium uptake by cardiac sarcoplasmic reticulum in human dilated cardiomyopathy. Cardiovasc. Res. 21, 601-605 (1987).

49. Dash, R., Frank, K. F., Carr, A. N., Moravec, C. S. \& Kranias, E. G. Gender influences on sarcoplasmic reticulum $\mathrm{Ca} 2+-$ handling in failing human myocardium. J. Mol. Cell. Cardiol. 33, 1345-1353 (2001).

50. BARKIHARRINGTON, L. Network integration of the adrenergic system in cardiac hypertrophy. Cardiovasc. Res. 63, 391-402 (2004).

51. Bibb, J. A. et al. Phosphorylation of Protein Phosphatase Inhibitor-1 by Cdk5. J. Biol. Chem. 276, 14490-14497 (2001).

52. Lipskaia, L., Chemaly, E. R., Hadri, L., Lompre, A. M. \& Hajjar, R. J. Sarcoplasmic reticulum Ca2 ATPase as a therapeutic target for heart failure. Expert Opinion on Biological Therapy vol. 10 29-41 (2010).

53. Cyganek, L. et al. Deep phenotyping of human induced pluripotent stem cell-derived atrial and ventricular cardiomyocytes. JCl insight 3, (2018).

54. Hung, V. et al. Spatially resolved proteomic mapping in living cells with the engineered peroxidase APEX2. Nat. Protoc. 11, 456-475 (2016).

55. Toischer, K. et al. Differential cardiac remodeling in preload versus afterload. Circulation 122, 993-1003 (2010).

56. Wagner, E., Brandenburg, S., Kohl, T. \& Lehnart, S. E. Analysis of Tubular Membrane Networks in Cardiac Myocytes from Atria and Ventricles. J. Vis. Exp. 1-19 (2014) doi:10.3791/51823.

57. Janssen, P. M. L. et al. The trabecula culture system: A novel technique to study contractile parameters over a multiday time period. Am. J. Physiol. - Hear. Circ. Physiol. 274, (1998).

58. Wittig, I., Braun, H.-P. \& Schägger, H. Blue native PAGE. Nat. Protoc. 1, 418-428 (2006).

59. Atanassov, I. \& Urlaub, H. Increased proteome coverage by combining PAGE and peptide isoelectric focusing: Comparative study of gel-based separation approaches. Proteomics 13, 2947-2955 (2013).

60. Chen, J., Bardes, E. E., Aronow, B. J. \& Jegga, A. G. ToppGene Suite for gene list enrichment analysis and candidate gene prioritization. Nucleic Acids Res. 37, W305W311 (2009). 
61. Alsina, K. M. et al. Loss of Protein Phosphatase 1 Regulatory Subunit PPP1R3A Promotes Atrial Fibrillation. Circulation 140, 681-693 (2019).

62. de Hoon, M. J. L., Imoto, S., Nolan, J. \& Miyano, S. Open source clustering software. Bioinformatics 20, 1453-1454 (2004).

63. Cuatrecasas, P. Protein purification by affinity chromatography. Derivatizations of agarose and polyacrylamide beads. J. Biol. Chem. 245, 3059-3065 (1970).

64. Young, K. H. Yeast Two-hybrid: So Many Interactions, (in) So Little Time.... Biol. Reprod. 58, 302-311 (1998).

65. Lam, S. S. et al. Directed evolution of APEX2 for electron microscopy and proximity labeling. Nat. Methods 12, 51-54 (2014).

66. Roux, K. J., Kim, D. I., Raida, M. \& Burke, B. A promiscuous biotin ligase fusion protein identifies proximal and interacting proteins in mammalian cells. J. Cell Biol. 196, 801810 (2012).

67. Roux, K. J., Kim, D. I. \& Burke, B. BioID: A Screen for Protein-Protein Interactions. Curr. Protoc. Protein Sci. 74, (2013).

68. Hung, V. et al. Proteomic Mapping of the Human Mitochondrial Intermembrane Space in Live Cells via Ratiometric APEX Tagging. Mol. Cell 55, 332-341 (2014).

69. Hung, V. et al. Proteomic mapping of cytosol-facing outer mitochondrial and ER membranes in living human cells by proximity biotinylation. Elife 6, 1-38 (2017).

70. Mick, D. U. et al. Proteomics of Primary Cilia by Proximity Labeling. Dev. Cell 35, 497512 (2015).

71. Lobingier, B. T. et al. An Approach to Spatiotemporally Resolve Protein Interaction Networks in Living Cells. Cell 169, 350-360.e12 (2017).

72. Wessels, H. J. C. T. et al. LC-MS/MS as an alternative for SDS-PAGE in blue native analysis of protein complexes. Proteomics 9, 4221-4228 (2009).

73. Wessels, H. J. C. T. et al. Analysis of 953 Human Proteins from a Mitochondrial HEK293 Fraction by Complexome Profiling. PLoS One 8, (2013).

74. Heide, H. et al. Complexome profiling identifies TMEM126B as a component of the mitochondrial complex i assembly complex. Cell Metab. 16, 538-549 (2012).

75. Camacho-Carvcajal, M. M., Wollscheid, B., Aebersold, R., Steimle, V. \& Schamel, W. W. A. Two-dimensional Blue Native/SDS gel electrophoresiss of multi-protein complexes from whole cellular lysates: A proteomics approach. Mol. Cell. Proteomics 3, 176-182 (2004).

76. Schägger, H. \& von Jagow, G. Blue native electrophoresis for isolation of membrane protein complexes in enzymatically active form. Anal. Biochem. 199, 223-231 (1991).

77. Giese, H. et al. NOVA: A software to analyze complexome profiling data. Bioinformatics 31, 440-441 (2015). 
78. Wigle, J. T. et al. Molecular cloning, expression, and chromosomal assignment of sarcolemmal-associated proteins. A family of acidic amphipathic alpha-helical proteins associated with the membrane. J. Biol. Chem. 272, 32384-94 (1997).

79. Wielowieyski, P. A. et al. Alternative splicing, expression, and genomic structure of the 3' region of the gene encoding the sarcolemmal-associated proteins (SLAPs) defines a novel class of coiled-coil tail-anchored membrane proteins. J. Biol. Chem. 275, 3847481 (2000).

80. Guzzo, R. M., Sevinc, S., Salih, M. \& Tuana, B. S. A novel isoform of sarcolemmal membrane-associated protein (SLMAP) is a component of the microtubule organizing centre. J. Cell Sci. 117, 2271-81 (2004).

81. Byers, J. T., Guzzo, R. M., Salih, M. \& Tuana, B. S. Hydrophobic profiles of the tail anchors in SLMAP dictate subcellular targeting. BMC Cell Biol. 10, 48 (2009).

82. Guzzo, R. M., Wigle, J., Salih, M., Moore, E. D. \& Tuana, B. S. Regulated expression and temporal induction of the tail-anchored sarcolemmal-membrane-associated protein is critical for myoblast fusion. Biochem. J. 381, 599-608 (2004).

83. Ishikawa, T. et al. A novel disease gene for Brugada syndrome: sarcolemmal membrane-associated protein gene mutations impair intracellular trafficking of hNav1.5. Circ. Arrhythm. Electrophysiol. 5, 1098-107 (2012).

84. Mlynarova, J. et al. SLMAP3 isoform modulates cardiac gene expression and function. PLoS One 14, e0214669 (2019).

85. Nader, M. et al. Tail-anchored membrane protein SLMAP is a novel regulator of cardiac function at the sarcoplasmic reticulum. Am. J. Physiol. Circ. Physiol. 302, H1138-H1145 (2012).

86. Nader, M. et al. SLMAP-3 is downregulated in human dilated ventricles and its overexpression promotes cardiomyocyte response to adrenergic stimuli by increasing intracellular calcium. Can. J. Physiol. Pharmacol. 97, 623-630 (2019).

87. Prior, K. K. et al. The endoplasmic reticulum chaperone calnexin is a NADPH oxidase NOX4 interacting protein. J. Biol. Chem. 291, 7045-7059 (2016).

88. Wittig, I., Beckhaus, T., Wumaier, Z., Karas, M. \& Schägger, H. Mass estimation of native proteins by blue native electrophoresis: principles and practical hints. Mol. Cell. Proteomics 9, 2149-61 (2010).

89. Guerrero-Castillo, S. et al. The Assembly Pathway of Mitochondrial Respiratory Chain Complex I. Cell Metab. 25, 128-139 (2017).

90. Geering, K. The functional role of $\beta$ subunits in oligomeric P-type ATPases. Journal of Bioenergetics and Biomembranes vol. 33 425-438 (2001).

91. Crambert, G., F̈uzesi, M., Garty, H., Karlish, S. \& Geering, K. Phospholemman (FXYD1) associates with $\mathrm{Na}, \mathrm{K}-\mathrm{AT}$ pase and regulates its transport properties. Proc. Natl. Acad. 
Sci. U. S. A. 99, 11476-11481 (2002).

92. Kranias, E. G. Regulation of Ca2+ transport by cyclic 3',5'-AMP-dependent and calciumcalmodulin-dependent phosphorylation of cardiac sarcoplasmic reticulum. BBA - Mol. Cell Res. 844, 193-199 (1985).

93. Brostrom, M. A., Reimann, E. M., Walsh, D. A. \& Krebs, E. G. A cyclic 3',5'-AMPstimulated protein kinase from cardiac muscle. Adv. Enzyme Regul. 8, 191-203 (1970).

94. Reimann, E. M., Brostrom, C. O., Corbin, J. D., King, C. A. \& Krebs, E. G. Separation of regulatory and catalytic subunits of the cyclic 3', 5'-adenosine monophosphatedependent protein kinase(s) of rabbit skeletal muscle. Biochem. Biophys. Res. Commun. 42, 187-194 (1971).

95. Baltas, L. G., Karczewski, P. \& Ernst-Georg, K. The cardiac sarcoplasmic reticulum phospholamban kinase is a distinct $\delta$-CaM kinase isozyme. FEBS Lett. 373, 71-75 (1995).

96. Guzzo, R. M., Salih, M., Moore, E. D. \& Tuana, B. S. Molecular properties of cardiac tail-anchored membrane protein SLMAP are consistent with structural role in arrangement of excitation-contraction coupling apparatus. Am. J. Physiol. Circ. Physiol. 288, H1810-H1819 (2005).

97. Kiriazis, $\mathrm{H}$. et al. Hypertrophy and functional alterations in hyperdynamic phospholamban-knockout mouse hearts under chronic aortic stenosis. Cardiovasc. Res. 53, 372-381 (2002).

98. Prévilon, M. et al. Comparative differential proteomic profiles of nonfailing and failing hearts after in vivo thoracic aortic constriction in mice overexpressing FKBP12.6. Physiol. Rep. 1, (2013).

99. Berrebi-Bertrand, I. et al. Biophysical interaction between phospholamban and protein phosphatase 1 regulatory subunit GM. FEBS Lett. 439, 224-230 (1998).

100. Müller, C. S. et al. High-Resolution Complexome Profiling by Cryoslicing BN-MS Analysis. J. Vis. Exp. (2019) doi:10.3791/60096.

101. Veltri, K. L., Espiritu, M. \& Singh, G. Distinct genomic copy number in mitochondria of different mammalian organs. J. Cell. Physiol. 143, 160-164 (1990).

102. Lopez-Crisosto, C. et al. Sarcoplasmic reticulum-mitochondria communication in cardiovascular pathophysiology. Nature Reviews Cardiology vol. 14 342-360 (2017).

103. Guskov, A. S. and A. An Overview of the Top Ten Detergents Used for Membrane Protein Crystallization. Crystals 7, 197 (2017).

104. Wöhlbrand, L. et al. Analysis of membrane-protein complexes of the marine sulfate reducer Desulfobacula toluolica Tol2 by $1 \mathrm{D}$ blue native-PAGE complexome profiling and 2D blue native-/SDS-PAGE. Proteomics 16, 973-988 (2016).

105. Senkler, J. et al. The mitochondrial complexome of Arabidopsis thaliana. Plant J. 89, 
1079-1092 (2017).

106. Heusel, M. et al. Complex-centric proteome profiling by SEC-SWATH-MS. Mol. Syst. Biol. 15, e8438 (2019).

107. Gardeitchik, T. et al. Bi-allelic Mutations in the Mitochondrial Ribosomal Protein MRPS2 Cause Sensorineural Hearing Loss, Hypoglycemia, and Multiple OXPHOS Complex Deficiencies. Am. J. Hum. Genet. 102, 685-695 (2018).

108. Vafiadaki, E., Arvanitis, D. A., Sanoudou, D., Kranias, E. G. \& Caramelli, D. Identification of a Protein Phosphatase-1/ Phospholamban Complex That Is Regulated by cAMPDependent Phosphorylation. PLoS One 8, (2013).

109. Colledge, M. \& Scott, J. D. AKAPs: From structure to function. Trends in Cell Biology vol. 9 216-221 (1999).

110. Herberg, F. W., Maleszka, A., Eide, T., Vossebein, L. \& Tasken, K. Analysis of A-kinase anchoring protein (AKAP) interaction with protein kinase a (PKA) regulatory subunits: PKA isoform specificity in AKAP binding. J. Mol. Biol. 298, 329-339 (2000).

111. Bers, D. M. Altered cardiac myocyte Ca regulation in heart failure. Physiology vol. 21 380-387 (2006).

112. Marks, A. R. Calcium cycling proteins and heart failure: mechanisms and therapeutics. J. Clin. Invest. 123, 46-52 (2013).

113. Biendarra-Tiegs, S. M. et al. Single-Cell RNA-Sequencing and Optical Electrophysiology of Human Induced Pluripotent Stem Cell-Derived Cardiomyocytes Reveal Discordance Between Cardiac Subtype-Associated Gene Expression Patterns and Electrophysiological Phenotypes. Stem Cells Dev. 28, 659-673 (2019).

114. Tang, Y. et al. Architecture, substructures, and dynamic assembly of STRIPAK complexes in Hippo signaling. Cell Discov. 5, 3 (2019).

115. Kim, D. I. et al. Probing nuclear pore complex architecture with proximity-dependent biotinylation. Proc. Natl. Acad. Sci. 111, E2453-E2461 (2014).

116. Kotani, N. et al. Biochemical visualization of cell surface molecular clustering in living cells. Proc. Natl. Acad. Sci. 105, 7405-7409 (2008).

117. Rhee, H. W. et al. Proteomic mapping of mitochondria in living cells via spatially restricted enzymatic tagging. Science (80-. ). 339, 1328-1331 (2013).

118. Bendayan, M. Worth its weight in gold. Science (80-. ). 291, 1363-1365 (2001).

119. Rybak, J. N., Scheurer, S. B., Neri, D. \& Elia, G. Purification of biotinylated proteins on streptavidin resin: A protocol for quantitative elution. Proteomics 4, 2296-2299 (2004).

120. Louch, W. E., Sheehan, K. A. \& Wolska, B. M. Methods in cardiomyocyte isolation, culture, and gene transfer. Journal of Molecular and Cellular Cardiology vol. 51 288298 (2011).

121. Wu, A. Z. et al. Phospholamban is concentrated in the nuclear envelope of 
cardiomyocytes and involved in perinuclear/nuclear calcium handling. J. Mol. Cell. Cardiol. 100, 1-8 (2016).

122. Hakem Zadeh, F. et al. AKAP6 and phospholamban colocalize and interact in HEK293T cells and primary murine cardiomyocytes. Physiol. Rep. 7, (2019).

123. Ong, S.-E. et al. Stable Isotope Labeling by Amino Acids in Cell Culture, SILAC, as a Simple and Accurate Approach to Expression Proteomics. Mol. Cell. Proteomics 1, 376386 (2002).

124. Feger, B. J. et al. Microgravity induces proteomics changes involved in endoplasmic reticulum stress and mitochondrial protection. Sci. Rep. 6, 34091 (2016).

125. MACDOUGALL, L. K., JONES, L. R. \& COHEN, P. Identification of the major protein phosphatases in mammalian cardiac muscle which dephosphorylate phospholamban. Eur. J. Biochem. 196, 725-734 (1991).

126. Kawai, M. et al. Ral GDP dissociation stimulator and Ral GTPase are involved in myocardial hypertrophy. Hypertens. (Dallas, Tex. 1979) 41, 956-62 (2003).

127. Christensen, G., Gruber, P. J., Wang, Y. \& Chien, K. R. Embryonic and neonatal cardiac gene transfer in vivo. Methods Mol. Biol. 219, 169-178 (2003).

128. Arakel, E. C. et al. Tuning the electrical properties of the heart by differential trafficking of KATP ion channel complexes. J. Cell Sci. 127, 2106-2119 (2014).

129. Sprenger, J. U. et al. In vivo model with targeted cAMP biosensor reveals changes in receptor-microdomain communication in cardiac disease. Nat. Commun. 6, 6965 (2015).

130. Li, T., Zhang, X., Jiang, K., Liu, J. \& Liu, Z. Dural effects of oxidative stress on cardiomyogenesis via Gata4 transcription and protein ubiquitination article. Cell Death Dis. 9, (2018).

131. Fernandez-Caggiano, M. et al. Oxidant-induced interprotein disulfide formation in cardiac protein DJ-1 occurs via an interaction with peroxiredoxin 2. J. Biol. Chem. 291, 10399-10410 (2016).

132. Chiang, D. Y., Heck, A. J. R., Dobrev, D. \& Wehrens, X. H. T. Regulating the regulator: Insights into the cardiac protein phosphatase 1 interactome. J. Mol. Cell. Cardiol. 101, 165-172 (2016).

133. DeGrande, S. T. et al. Molecular mechanisms underlying cardiac protein phosphatase 2A regulation in heart. J. Biol. Chem. 288, 1032-1046 (2013).

134. Yang, $X$. et al. Structural basis for protein-protein interactions in the 14-3-3 protein family. Proc. Natl. Acad. Sci. 103, 17237-17242 (2006).

135. Yaffe, M. B. et al. The structural basis for 14-3-3:phosphopeptide binding specificity. Cell (1997) doi:10.1016/S0092-8674(00)80487-0.

136. Wilkert, E. W., Grant, R. A., Artim, S. C. \& Yaffe, M. B. A structural basis for 14-3-30 
functional specificity. J. Biol. Chem. 280, 18891-18898 (2005).

137. Madeira, F. et al. 14-3-3-Pred: improved methods to predict 14-3-3-binding phosphopeptides. Bioinformatics 31, 2276-2283 (2015).

138. Schröder, E., Brennan, J. P. \& Eaton, P. Cardiac peroxiredoxins undergo complex modifications during cardiac oxidant stress. Am. J. Physiol. Circ. Physiol. 295, H425H433 (2008).

139. Kang, S. W., Rhee, S. G., Chang, T. S., Jeong, W. \& Choi, M. H. 2-Cys peroxiredoxin function in intracellular signal transduction: Therapeutic implications. Trends in Molecular Medicine (2005) doi:10.1016/j.molmed.2005.10.006.

140. Chu, G. et al. Proteomic analysis of hyperdynamic mouse hearts with enhanced sarcoplasmic reticulum calcium cycling. FASEB J. 18, 1725-1727 (2004).

141. Zhao, W. et al. Protection of peroxiredoxin II on oxidative stress-induced cardiomyocyte death and apoptosis. Basic Res. Cardiol. 104, 377-389 (2009).

142. Wen Zhao, Xiaojing Shi, Wenjuan Zhou, Huimin Wang, Xuepeng Geng, Shuang Zhang , Jingjing Wang, Evangelia G Kranias, and H.-M. L. Abstract 276: The Regulatory Effects Of Peroxiredoxin II On Phospholamban Phosphorylation And Cardiac Contractile Function In Vivo | Circulation Research. Circulation Research. 2014;115:A276 https://www.ahajournals.org/doi/abs/10.1161/res.115.suppl_1.276 (2014).

143. Li, A., Ponten, F. \& dos Remedios, C. G. The interactome of LIM domain proteins: The contributions of LIM domain proteins to heart failure and heart development. Proteomics 12, 203-225 (2012).

144. Dawid, I. B., Breen, J. J. \& Toyama, R. LIM domains: multiple roles as adapters and functional modifiers in protein interactions. Trends Genet. 14, 156-62 (1998).

145. Beise, N. \& Trimble, W. Septins at a glance. J. Cell Sci. 124, 4141-4146 (2011).

146. Ahuja, P., Perriard, E., Trimble, W., Perriard, J. C. \& Ehler, E. Probing the role of septins in cardiomyocytes. Exp. Cell Res. 312, 1598-1609 (2006).

147. Bidwell, P. A., Haghighi, K. \& Kranias, E. G. The antiapoptotic protein HAX-1 mediates half of phospholamban's inhibitory activity on calcium cycling and contractility in the heart. (2017) doi:10.1074/jbc.RA117.000128.

148. Balcerak, A. et al. HAX1 impact on collective cell migration, cell adhesion, and cell shape is linked to the regulation of actomyosin contractility. Mol. Biol. Cell 30, 30243036 (2019).

149. Schaub, M. C. \& Perry, S. V. The relaxing protein system of striated muscle. Resolution of the troponin complex into inhibitory and calcium ion-sensitizing factors and their relationship to tropomyosin. Biochem. J. 115, 993-1004 (1969).

150. Ebashi, S., Ohtsuki, I. \& Mihashi, K. Regulatory Proteins of Muscle with Special 
Reference to Troponin. Cold Spring Harb. Symp. Quant. Biol. 37, 215-223 (1973).

151. Ebashi, S. \& Endo, M. Calcium and muscle contraction. Progress in Biophysics and Molecular Biology vol. 18 (1968).

152. Kranias, E. G. \& Solaro, R. J. Phosphorylation of troponin I and phospholamban during catecholamine stimulation of rabbit heart. Nature 298, 182-184 (1982).

153. Li, L., Desantiago, J., Chu, G., Kranias, E. G. \& Bers, D. M. Phosphorylation of phospholamban and troponin $\mathrm{I}$ in $\beta$-adrenergic-induced acceleration of cardiac relaxation. Am. J. Physiol. - Hear. Circ. Physiol. 278, (2000).

154. Dreiza, C. M. et al. The small heat shock protein, HSPB6, in muscle function and disease. Cell Stress and Chaperones vol. 15 1-11 (2010). 


\section{Appendix}

Table 9 APEX2-PLN enriched proteins after PLN targeted proximity labeling

\begin{tabular}{|c|c|c|c|c|c|c|c|}
\hline$\#$ & $\begin{array}{l}\text { Protein } \\
\text { IDs }\end{array}$ & Protein names & $\begin{array}{c}\text { Gene } \\
\text { names }\end{array}$ & $\begin{array}{l}\text { APEX2-PLN } \\
\text { / eGFP } \\
\text { Ratio } \\
\text { normalized } \\
\text { mean }\end{array}$ & $\begin{array}{l}\text { APEX2-PLN/ } \\
\text { APEX2- } \\
\text { PLN } \Delta(1-29) \\
\text { Ratio } \\
\text { normalized } \\
\text { mean }\end{array}$ & $\begin{array}{l}\text { APEX2- } \\
\text { PLN / } \\
\text { eGFP } \\
\text { z-test }\end{array}$ & $\begin{array}{c}\text { APEX2-PLN } \\
\text { APEX2- } \\
\text { PLNA(1-29) } \\
\text { z-test }\end{array}$ \\
\hline 1 & Q9Z269 & $\begin{array}{l}\text { Vesicle-associated membrane } \\
\text { protein-associated protein B }\end{array}$ & Vapb & 2.06 & 0.19 & $9.82 E-37$ & $3.25 \mathrm{E}-15$ \\
\hline 2 & Q01728-7 & Sodium/calcium exchanger 1 & Slc8a1 & 2.26 & 0.54 & $2.50 \mathrm{E}-05$ & $1.07 \mathrm{E}-11$ \\
\hline 3 & P61016 & Cardiac phospholamban & PIn & 3.08 & 4.69 & $9.03 E-23$ & $2.94 \mathrm{E}-10$ \\
\hline 4 & P45592 & Cofilin-1 & Cfl1 & 0.86 & 0.30 & $9.90 \mathrm{E}-36$ & 4.57E-08 \\
\hline 5 & B5DEN4 & L-lactate dehydrogenase & Ldha & 0.44 & 0.16 & $2.70 \mathrm{E}-02$ & 5.73E-07 \\
\hline 6 & A0JPQ4 & $\begin{array}{l}\text { Tripartite motif-containing protein } \\
72\end{array}$ & Trim72 & 1.83 & 0.32 & 4.95E-14 & 2.33E-06 \\
\hline 7 & Q9EPF2 & Cell surface glycoprotein MUC18 & Mcam & 1.05 & 0.69 & 1.75E-09 & 1.31E-05 \\
\hline 8 & F1LYS7 & Sarcoglycan, delta & Sgcd & 2.00 & 0.35 & $6.74 \mathrm{E}-28$ & $2.86 \mathrm{E}-05$ \\
\hline 9 & G3V913 & Heat shock protein beta- 1 & Hspb1 & 1.28 & 0.22 & $6.10 \mathrm{E}-23$ & $2.97 \mathrm{E}-05$ \\
\hline 10 & Q5XFX0 & Transgelin-2 & Tagln2 & 2.21 & 0.58 & $8.74 \mathrm{E}-47$ & $3.27 \mathrm{E}-05$ \\
\hline 11 & Q6P6U2 & $\begin{array}{l}26 \mathrm{~S} \text { protease regulatory subunit } \\
6 \mathrm{~A}\end{array}$ & Psmc3 & 1.72 & 0.25 & 4.32E-14 & 7.12E-05 \\
\hline 12 & Q5XID6 & Sarcoglycan, gamma & Sgcg & 1.98 & 0.24 & $2.02 E-25$ & $1.25 \mathrm{E}-04$ \\
\hline 13 & G3V7U0 & $\begin{array}{l}\text { Cysteine and glycine-rich protein } \\
3\end{array}$ & Csrp3 & 2.34 & 0.40 & $4.22 \mathrm{E}-29$ & 2.30E-04 \\
\hline 14 & F7EMB2 & $\begin{array}{l}\text { RNA transcription, translation } \\
\text { and transport factor }\end{array}$ & $\begin{array}{l}\text { RGD130 } \\
4704\end{array}$ & 1.59 & 0.31 & $9.20 \mathrm{E}-22$ & 2.34E-04 \\
\hline 15 & Q08163 & \begin{tabular}{ll|} 
Adenylyl & cyclase-associated \\
protein 1 &
\end{tabular} & Cap1 & 1.70 & 0.49 & 1.12E-39 & $3.16 \mathrm{E}-04$ \\
\hline 16 & P07943 & Aldose reductase & Akr1b1 & 2.18 & 0.37 & $1.28 \mathrm{E}-10$ & 3.71E-04 \\
\hline 17 & Q8R3Z7 & EH-domain-containing 4 & Ehd4 & 1.86 & 0.64 & $9.15 \mathrm{E}-30$ & $4.16 \mathrm{E}-04$ \\
\hline 18 & P61589 & Transforming protein RhoA & Rhoa & 0.44 & 0.33 & $2.16 \mathrm{E}-08$ & $4.44 \mathrm{E}-04$ \\
\hline 19 & P11762 & Galectin-1 & Lgals1 & 1.15 & 0.44 & $2.53 \mathrm{E}-33$ & $6.90 \mathrm{E}-04$ \\
\hline 20 & P11232 & Thioredoxin & Txn1 & 1.63 & 0.22 & $2.21 \mathrm{E}-07$ & $7.05 \mathrm{E}-04$ \\
\hline 21 & P48500 & Triosephosphate isomerase & Tpi1 & 2.45 & 0.59 & $1.04 \mathrm{E}-06$ & $8.55 \mathrm{E}-04$ \\
\hline 22 & P47875 & $\begin{array}{l}\text { Cysteine and glycine-rich protein } \\
1\end{array}$ & Csrp1 & 1.88 & 0.66 & $1.98 \mathrm{E}-03$ & $1.02 E-03$ \\
\hline
\end{tabular}




\begin{tabular}{|c|c|c|c|c|c|c|c|}
\hline 23 & P27605 & $\begin{array}{l}\text { Hypoxanthine-guanine } \\
\text { phosphoribosyltransferase }\end{array}$ & Hprt1 & 2.00 & 0.41 & $1.75 \mathrm{E}-15$ & 1.06E-03 \\
\hline 24 & $\begin{array}{l}\text { A0A0G2K } \\
1 Y 8\end{array}$ & $\begin{array}{l}\text { Spectrin alpha chain, non- } \\
\text { erythrocytic } 1\end{array}$ & Sptan1 & 1.57 & 0.78 & 2.14E-03 & $1.12 \mathrm{E}-03$ \\
\hline 25 & P23693 & Troponin I, cardiac muscle & Tnni3 & 2.83 & 0.60 & $1.51 \mathrm{E}-28$ & $1.20 \mathrm{E}-03$ \\
\hline 26 & Q9Z2J4 & Nexilin & Nexn & 1.45 & 0.56 & $9.86 \mathrm{E}-10$ & $1.38 \mathrm{E}-03$ \\
\hline 27 & A2RUW1 & Toll-interacting protein & Tollip & 1.71 & 0.53 & 2.30E-03 & $1.46 \mathrm{E}-03$ \\
\hline 28 & P68255 & 14-3-3 protein theta & Ywhaq & 2.37 & 0.54 & $4.76 \mathrm{E}-30$ & $1.68 \mathrm{E}-03$ \\
\hline 29 & G3V7Q7 & $\begin{array}{l}\text { IQ motif containing GTPase } \\
\text { activating protein } 1 \text { (Predicted) }\end{array}$ & lqgap1 & 1.97 & 0.62 & 8.17E-04 & 1.87E-03 \\
\hline 30 & Q08290 & Calponin-1 & Cnn1 & 1.69 & 0.74 & $2.57 \mathrm{E}-61$ & 1.92E-03 \\
\hline 31 & $\begin{array}{l}\text { A0A0G2K } \\
3 Z 9\end{array}$ & Peroxiredoxin-1 & Prdx1 & 2.31 & 0.31 & 1.35E-25 & 2.04E-03 \\
\hline 32 & B2GVB4 & Septin-9 & Sept9 & 1.28 & 1.01 & 2.93E-10 & $2.20 \mathrm{E}-03$ \\
\hline 33 & F1LMC7 & Septin-7 & Sept7 & 2.48 & 0.74 & $5.90 \mathrm{E}-15$ & $2.27 \mathrm{E}-03$ \\
\hline 34 & P97541 & Heat shock protein beta- 6 & Hspb6 & 1.25 & 0.47 & 1.90E-03 & $2.45 \mathrm{E}-03$ \\
\hline 35 & Q4G079 & $\begin{array}{l}\text { Aminoacyl tRNA synthetase } \\
\text { complex-interacting } \\
\text { multifunctional protein } 1\end{array}$ & Aimp1 & 1.80 & 0.25 & 5.57E-12 & $2.60 \mathrm{E}-03$ \\
\hline 36 & D3ZW08 & Adenylosuccinate lyase & Adsl & 1.56 & 0.26 & $4.93 \mathrm{E}-19$ & $2.79 \mathrm{E}-03$ \\
\hline 37 & $\begin{array}{l}\text { A0A0G2K } \\
174\end{array}$ & LIM domain 7 & Lmo7 & 1.45 & 0.29 & 3.88E-03 & 2.92E-03 \\
\hline 38 & Q9Z0V5 & Peroxiredoxin-4 & Prdx4 & 0.85 & 0.48 & $3.23 E-04$ & $3.21 \mathrm{E}-03$ \\
\hline 39 & $\begin{array}{l}\text { A0A0G2J } \\
\text { UL7 }\end{array}$ & Septin-11 & Sept11 & 0.97 & 0.88 & 3.92E-02 & 3.53E-03 \\
\hline 40 & $\begin{array}{l}\text { A0A0G2J } \\
\text { WK7 }\end{array}$ & Transgelin & Tagln & 2.64 & 0.60 & $\begin{array}{c}7.72 \mathrm{E}- \\
101\end{array}$ & 3.67E-03 \\
\hline 41 & P63102 & 14-3-3 protein zeta/delta & Ywhaz & 1.78 & 0.44 & 7.56E-36 & 3.76E-03 \\
\hline 42 & Q2IBC6 & Caveolin & Cav1 & 1.49 & 0.38 & 9.82E-05 & 3.97E-03 \\
\hline 43 & P62142 & $\begin{array}{l}\text { Serine/threonine-protein } \\
\text { phosphatase PP1-beta catalytic } \\
\text { subunit }\end{array}$ & Ppp1cb & 0.80 & 0.26 & 1.42E-07 & 4.03E-03 \\
\hline 44 & Q6P9V1 & Tetraspanin & Cd81 & 1.66 & 0.24 & 2.51E-04 & $4.26 \mathrm{E}-03$ \\
\hline 45 & P40329 & $\begin{array}{l}\text { Arginine--tRNA } \\
\text { cytoplasmic }\end{array}$ & Rars & 1.05 & 0.30 & 6.81E-06 & 4.33E-03 \\
\hline 46 & P37397 & Calponin-3 & Cnn3 & 1.71 & 0.53 & $4.85 \mathrm{E}-33$ & $4.46 \mathrm{E}-03$ \\
\hline 47 & $\begin{array}{l}\text { A0A0G2K } \\
013\end{array}$ & Alpha-actinin-4 & Actn4 & 1.50 & 0.56 & $4.60 \mathrm{E}-14$ & 4.48E-03 \\
\hline
\end{tabular}




\begin{tabular}{|c|c|c|c|c|c|c|c|}
\hline 48 & $\begin{array}{l}\text { AOA0G2K } \\
1 \mathrm{CO}\end{array}$ & Actin-related protein 3 & Actr3 & 1.38 & 0.25 & $1.45 E-24$ & 4.66E-03 \\
\hline 49 & P68511 & 14-3-3 protein eta & Ywhah & 1.41 & 0.45 & $7.52 \mathrm{E}-11$ & $5.05 \mathrm{E}-03$ \\
\hline 50 & Q9ESH1 & $26 \mathrm{~S}$ proteasome subunit S5a & Psmd4 & 0.90 & 0.10 & 4.79E-03 & $5.06 \mathrm{E}-03$ \\
\hline 51 & O35878 & Heat shock protein beta-2 & Hspb2 & 2.17 & 0.22 & $2.28 \mathrm{E}-27$ & $5.31 \mathrm{E}-03$ \\
\hline 52 & Q6T487 & Alpha-actinin-1 & Actn1 & 1.07 & 0.54 & 1.44E-09 & 5.46E-03 \\
\hline 53 & P62963 & Profilin-1 & Pfn1 & 1.70 & 0.37 & $3.63 \mathrm{E}-11$ & $5.48 \mathrm{E}-03$ \\
\hline 54 & Q99MZ8 & LIM and SH3 domain protein 1 & Lasp1 & 1.43 & 0.67 & 4.56E-09 & $5.70 \mathrm{E}-03$ \\
\hline 55 & P85515 & Alpha-centractin & Actr1a & 0.98 & 0.16 & $2.41 \mathrm{E}-06$ & 5.73E-03 \\
\hline 56 & P62260 & 14-3-3 protein epsilon & Ywhae & 1.81 & 0.40 & $1.29 \mathrm{E}-19$ & $5.90 \mathrm{E}-03$ \\
\hline 57 & B4F761 & Monocarboxylate transporter 4 & Slc16a3 & 0.72 & 0.28 & 1.17E-09 & $5.90 \mathrm{E}-03$ \\
\hline 58 & P52944 & PDZ and LIM domain protein 1 & Pdlim1 & 2.13 & 0.45 & $3.25 \mathrm{E}-11$ & $6.05 \mathrm{E}-03$ \\
\hline 59 & Q4PP99 & Cardiac troponin C & Tnnc1 & 1.82 & 0.30 & 8.99E-06 & $6.19 \mathrm{E}-03$ \\
\hline 60 & P05065 & $\begin{array}{l}\text { Fructose-bisphosphate aldolase } \\
\text { A }\end{array}$ & Aldoa & 3.19 & 0.62 & 2.62E-08 & $6.28 \mathrm{E}-03$ \\
\hline 61 & F7EPH4 & Inorganic pyrophosphatase 1 & Ppa1 & 1.06 & 0.40 & $2.09 \mathrm{E}-51$ & $6.64 \mathrm{E}-03$ \\
\hline 62 & P04636 & $\begin{array}{ll}\text { Malate } & \text { dehydrogenase, } \\
\text { mitochondrial } & \end{array}$ & Mdh2 & 1.39 & 0.88 & $1.55 \mathrm{E}-02$ & $6.75 \mathrm{E}-03$ \\
\hline 63 & $\begin{array}{l}\text { A0A0G2JS } \\
\text { H9 }\end{array}$ & Peroxiredoxin-2 & Prdx2 & 2.61 & 0.23 & 1.67E-25 & $6.80 \mathrm{E}-03$ \\
\hline 64 & C0JPT7 & Filamin A & Flna & 2.13 & 0.53 & $3.18 \mathrm{E}-04$ & $6.84 \mathrm{E}-03$ \\
\hline 65 & D3ZVQ0 & $\begin{array}{l}\text { Ubiquitin } \quad \text { carboxyl-terminal } \\
\text { hydrolase }\end{array}$ & Usp5 & 1.10 & 0.33 & 2.05E-06 & $7.12 \mathrm{E}-03$ \\
\hline 66 & P38983 & $40 \mathrm{~S}$ ribosomal protein $\mathrm{SA}$ & Rpsa & 1.06 & 0.16 & $3.73 E-03$ & $7.27 \mathrm{E}-03$ \\
\hline 67 & P61983 & 14-3-3 protein gamma & Ywhag & 1.75 & 0.40 & $1.57 \mathrm{E}-13$ & 7.74E-03 \\
\hline 68 & Q5XI34 & $\begin{array}{l}\text { Protein phosphatase } 2 \text { (Formerly } \\
2 A) \text {, regulatory subunit } A(P R 65), \\
\text { alpha isoform }\end{array}$ & Ppp2r1a & 2.91 & 0.20 & $4.78 \mathrm{E}-03$ & 7.83E-03 \\
\hline 69 & P52481 & $\begin{array}{l}\text { Adenylyl } \quad \text { cyclase-associated } \\
\text { protein } 2\end{array}$ & Cap2 & 1.57 & 0.32 & 1.36E-36 & 8.36E-03 \\
\hline 70 & Q66H61 & Glutamine--tRNA ligase & $\begin{array}{l}\text { RGD156 } \\
2301\end{array}$ & 0.99 & 0.08 & 6.67E-12 & 8.57E-03 \\
\hline 71 & Q91Y81 & Septin-2 & Sept2 & 1.46 & 0.69 & 1.81E-06 & $8.94 \mathrm{E}-03$ \\
\hline 72 & Q5RKI5 & FLII, actin-remodeling protein & Flii & 1.65 & 0.28 & 1.95E-02 & $9.23 \mathrm{E}-03$ \\
\hline 73 & Q32PX2 & $\begin{array}{l}\text { Aminoacyl tRNA synthase } \\
\text { complex-interacting } \\
\text { multifunctional protein } 2\end{array}$ & Aimp2 & 1.84 & 0.22 & 3.03E-31 & $9.26 \mathrm{E}-03$ \\
\hline
\end{tabular}




\begin{tabular}{|c|c|c|c|c|c|c|c|}
\hline 74 & |F1M978 & | Inositol monophosphatase 1 & Impa1 & 1.67 & 0.52 & $1.58 \mathrm{E}-07$ & 9.73E-03 \\
\hline 75 & $\begin{array}{l}\text { A0A0G2JS } \\
\text { S8 }\end{array}$ & Peroxiredoxin-5, mitochondrial & Prdx5 & 2.50 & 0.22 & $3.10 \mathrm{E}-11$ & 9.73E-03 \\
\hline 76 & Q99J82 & Integrin-linked protein kinase & Ilk & 2.11 & 0.46 & $3.10 \mathrm{E}-20$ & $1.02 \mathrm{E}-02$ \\
\hline 77 & P63170 & Dynein light chain 1, cytoplasmic & Dynll1 & 2.03 & 0.26 & $2.70 \mathrm{E}-19$ & 1.09E-02 \\
\hline 78 & G3V7I0 & $\begin{array}{l}\text { Thioredoxin-dependent peroxide } \\
\text { reductase, mitochondrial }\end{array}$ & Prdx3 & 1.26 & 0.39 & $1.14 \mathrm{E}-07$ & 1.13E-02 \\
\hline 79 & A9YUA5 & Troponin T, cardiac muscle & Tnnt2 & 2.95 & 0.37 & $\begin{array}{c}7.90 \mathrm{E}- \\
105\end{array}$ & $1.21 \mathrm{E}-02$ \\
\hline 80 & P14668 & Annexin A5 & Anxa5 & 2.62 & 0.64 & 3.32E-13 & $1.23 \mathrm{E}-02$ \\
\hline 81 & P97536 & $\begin{array}{ll}\text { Cullin-associated } & \text { NEDD8- } \\
\text { dissociated protein } 1 & \end{array}$ & Cand1 & 0.66 & 0.35 & 4.69E-03 & 1.27E-02 \\
\hline 82 & G3V8B0 & Myosin-7 & Myh7 & 1.94 & 0.33 & 7.89E-04 & $1.29 \mathrm{E}-02$ \\
\hline 83 & $\begin{array}{l}\text { A0A140TA } \\
\text { A4 }\end{array}$ & $\begin{array}{l}\text { Programmed cell death 6- } \\
\text { interacting protein }\end{array}$ & Pdcd6ip & 1.04 & 0.19 & 1.33E-05 & $1.31 \mathrm{E}-02$ \\
\hline 84 & Q6AYF2 & LIM and cysteine-rich domains 1 & Lmcd1 & 1.19 & 0.45 & $1.59 \mathrm{E}-14$ & $1.36 \mathrm{E}-02$ \\
\hline 85 & G3V852 & $\begin{array}{l}\text { RCG55135, isoform CRA_b } \\
\text { (Talin 1) }\end{array}$ & TIn1 & 2.19 & 0.44 & 7.25E-04 & $1.42 \mathrm{E}-02$ \\
\hline 86 & F1LZX9 & Integrin subunit alpha V & Itgav & 1.55 & 0.58 & $8.32 E-08$ & $1.45 \mathrm{E}-02$ \\
\hline 87 & R9PXU3 & $\begin{array}{l}\text { Protein kinase } \mathrm{C} \text { and casein } \\
\text { kinase substrate in neurons } 2 \\
\text { protein }\end{array}$ & Pacsin2 & 1.62 & 0.50 & $2.22 \mathrm{E}-12$ & $1.53 \mathrm{E}-02$ \\
\hline 88 & D4A7U1 & Zyxin & Zyx & 1.66 & 0.54 & $2.57 \mathrm{E}-23$ & 1.63E-02 \\
\hline 89 & Q5VLR5 & BWK4 & Erp44 & 0.88 & 0.27 & $1.01 \mathrm{E}-05$ & $1.84 \mathrm{E}-02$ \\
\hline 90 & Q6RUV5 & $\begin{array}{l}\text { Ras-related C3 botulinum toxin } \\
\text { substrate } 1\end{array}$ & Rac1 & 0.56 & 0.21 & $3.86 \mathrm{E}-14$ & 1.93E-02 \\
\hline 91 & P31399 & $\begin{array}{l}\text { ATP synthase subunit } \mathrm{d} \text {, } \\
\text { mitochondrial }\end{array}$ & Atp5h & 1.46 & 0.43 & 3.11E-06 & 1.96E-02 \\
\hline 92 & Q5XIM9 & T-complex protein 1 subunit beta & Cct2 & 1.27 & 0.42 & $2.17 \mathrm{E}-38$ & $1.98 \mathrm{E}-02$ \\
\hline 93 & Q9Z270 & $\begin{array}{l}\text { Vesicle-associated membrane } \\
\text { protein-associated protein A }\end{array}$ & Vapa & 1.91 & 0.13 & $3.02 E-25$ & 2.10E-02 \\
\hline 94 & D3ZZZ9 & $\begin{array}{l}\text { Catenin (Cadherin associated } \\
\text { protein), delta } 1 \text { (Predicted) }\end{array}$ & Ctnnd1 & 0.68 & 0.45 & 3.74E-02 & 2.11E-02 \\
\hline 95 & BOBNA7 & $\begin{array}{l}\text { Eukaryotic translation initiation } \\
\text { factor } 3 \text { subunit I }\end{array}$ & Eif3i & 0.84 & 0.24 & $1.84 \mathrm{E}-03$ & 2.19E-02 \\
\hline 96 & G3V6S0 & Spectrin beta chain & Sptbn1 & 2.20 & 0.69 & $3.00 \mathrm{E}-05$ & $2.31 \mathrm{E}-02$ \\
\hline 97 & M0R3V7 & Sodium/calcium exchanger 1 & Slc8a1 & 1.15 & 0.77 & $4.29 \mathrm{E}-04$ & $2.40 \mathrm{E}-02$ \\
\hline 98 & P07335 & Creatine kinase B-type & Ckb & 1.62 & 0.54 & $4.86 \mathrm{E}-03$ & $2.41 \mathrm{E}-02$ \\
\hline
\end{tabular}




\begin{tabular}{|c|c|c|c|c|c|c|c|}
\hline 99 & $\begin{array}{l}\text { A0A0G2K } \\
\text { 4D0 }\end{array}$ & Ankyrin-3 & Ank3 & 1.46 & 0.48 & 8.05E-03 & $2.50 \mathrm{E}-02$ \\
\hline 100 & P63259 & Actin, cytoplasmic 2 & Actg1 & 1.09 & 0.34 & $2.32 \mathrm{E}-06$ & $2.54 \mathrm{E}-02$ \\
\hline 101 & Q4FZT9 & $\begin{array}{l}26 \mathrm{~S} \text { proteasome non-ATPase } \\
\text { regulatory subunit } 2\end{array}$ & Psmd2 & 0.75 & 0.16 & 8.12E-09 & 2.59E-02 \\
\hline 102 & D4A6X1 & Dysferlin (Predicted) & Dysf & 1.48 & 0.26 & $2.29 \mathrm{E}-03$ & $2.63 E-02$ \\
\hline 103 & D3ZRX9 & Calponin & Cnn2 & 1.43 & 0.42 & 7.59E-06 & 2.65E-02 \\
\hline 104 & Q07936 & Annexin A2 & Anxa2 & 2.12 & 0.41 & $3.17 \mathrm{E}-10$ & $2.77 \mathrm{E}-02$ \\
\hline 105 & $\begin{array}{l}\text { A0A0H2U } \\
\mathrm{HR} 7\end{array}$ & Filamin-C & Flnc & 2.33 & 0.19 & 1.07E-03 & 2.87E-02 \\
\hline 106 & Q9QZA6 & CD151 antigen & Cd151 & 1.92 & 0.25 & $2.78 \mathrm{E}-06$ & $2.90 \mathrm{E}-02$ \\
\hline 107 & P52555 & $\begin{array}{l}\text { Endoplasmic reticulum resident } \\
\text { protein } 29\end{array}$ & Erp29 & 0.65 & 0.38 & $3.24 \mathrm{E}-05$ & 2.94E-02 \\
\hline 108 & P60711 & Actin, cytoplasmic 1 & Actb & 1.24 & 0.34 & $9.18 \mathrm{E}-15$ & $2.97 \mathrm{E}-02$ \\
\hline 109 & D4ACB8 & $\begin{array}{llll}\text { Chaperonin } & \text { subunit } & 8 & \text { (Theta) } \\
\text { (Predicted) } & & & \\
\end{array}$ & Cct8 & 1.50 & 0.11 & 2.51E-39 & 3.00E-02 \\
\hline 110 & Q5EBB0 & 14-3-3 sigma & Sfn & 2.33 & 0.65 & 1.16E-06 & $3.27 \mathrm{E}-02$ \\
\hline 111 & $\begin{array}{l}\text { A0A0G2J } \\
\text { UX5 }\end{array}$ & $\begin{array}{l}\text { Transcriptional activator protein } \\
\text { Pur-beta }\end{array}$ & Purb & 1.16 & 0.15 & $1.00 \mathrm{E}-11$ & 3.32E-02 \\
\hline 112 & P26772 & $\begin{array}{l}10 \mathrm{kDa} \text { heat shock protein, } \\
\text { mitochondrial }\end{array}$ & Hspe1 & 2.58 & 1.24 & 5.27E-07 & $3.40 \mathrm{E}-02$ \\
\hline 113 & R9PXU6 & Vinculin & $\mathrm{Vcl}$ & 1.55 & 0.31 & 1.10E-02 & $3.43 E-02$ \\
\hline 114 & G3V936 & Citrate synthase & Cs & 0.91 & 0.37 & 3.01E-02 & $3.50 \mathrm{E}-02$ \\
\hline 115 & $\begin{array}{l}\text { A0A0G2K } \\
9 A 2\end{array}$ & $\begin{array}{l}\text { Actin-related protein } 2 / 3 \text { complex } \\
\text { subunit } 2\end{array}$ & Arpc2 & 0.98 & 0.16 & $3.21 E-15$ & 3.71E-02 \\
\hline 116 & Q6P7A7 & $\begin{array}{l}\text { Dolichyl- } \\
\text { diphosphooligosaccharide-- } \\
\text { protein } \quad \text { glycosyltransferase } \\
\text { subunit } 1\end{array}$ & Rpn1 & 0.35 & 0.14 & $4.28 \mathrm{E}-02$ & 3.76E-02 \\
\hline 117 & G3V9E3 & Non-muscle caldesmon & Cald1 & 0.87 & 0.49 & $9.61 \mathrm{E}-04$ & $3.82 \mathrm{E}-02$ \\
\hline 118 & $\begin{array}{l}\text { A0A0G2K } \\
\text { AQ5 }\end{array}$ & Myozenin 2 (Predicted) & Myoz2 & 2.26 & 0.30 & $1.50 \mathrm{E}-34$ & 3.91E-02 \\
\hline 119 & $\begin{array}{l}\text { A0A0G2JS } \\
M 3\end{array}$ & PDZ and LIM domain protein 3 & Pdlim3 & 2.51 & 0.36 & 7.04E-07 & 3.92E-02 \\
\hline 120 & G3V6S3 & Calumenin & Calu & 0.60 & 0.23 & 1.09E-02 & $3.93 E-02$ \\
\hline 121 & G3V7Y3 & $\begin{array}{l}\text { ATP synthase subunit delta, } \\
\text { mitochondrial }\end{array}$ & Atp5d & 0.43 & 0.60 & 1.76E-02 & 3.99E-02 \\
\hline 122 & Q9ESN0 & Protein Niban & Fam129a & 0.68 & 0.27 & 9.99E-03 & 4.10E-02 \\
\hline
\end{tabular}




\begin{tabular}{|c|c|c|c|c|c|c|c|}
\hline 123 & | D3ZGE6 & Src substrate cortactin & Cttn & 1.15 & 0.33 & 6.07E-04 & 4.13E-02 \\
\hline 124 & P14408-2 & $\begin{array}{ll}\text { Fumarate } & \text { hydratase, } \\
\text { mitochondrial } & \end{array}$ & $\mathrm{Fh}$ & 0.52 & 0.34 & 1.74E-02 & 4.17E-02 \\
\hline 125 & D3ZG43 & \begin{tabular}{ll} 
NADH & \multicolumn{2}{r}{ dehydrogenase } \\
(Ubiquinone) & Fe-S protein 3 \\
(Predicted) &
\end{tabular} & Ndufs3 & 1.95 & 0.52 & 2.45E-06 & 4.26E-02 \\
\hline 126 & $\begin{array}{l}\text { A0A0G2K } \\
542\end{array}$ & $\begin{array}{l}\text { UTP--glucose-1-phosphate } \\
\text { uridylyltransferase }\end{array}$ & Ugp2 & 1.81 & 0.29 & 1.99E-57 & 4.29E-02 \\
\hline 127 & Q6AYD5 & $\begin{array}{l}\text { Eukaryotic peptide chain release } \\
\text { factor GTP-binding subunit } \\
\text { ERF3B-like }\end{array}$ & Gspt1 & 0.57 & 0.17 & 4.78E-02 & 4.45E-02 \\
\hline 128 & P18163 & $\begin{array}{l}\text { Long-chain-fatty-acid--CoA } \\
\text { ligase } 1\end{array}$ & Acsl1 & 0.42 & 0.34 & 4.69E-02 & 4.50E-02 \\
\hline 129 & G3V8A5 & $\begin{array}{l}\text { Vacuolar protein } \\
\text { associated protein } 35\end{array}$ & Vps35 & 0.48 & 0.35 & $1.52 \mathrm{E}-06$ & 4.70E-02 \\
\hline 130 & P82995 & Heat shock protein HSP 90-alpha & $\begin{array}{l}\text { Hsp90aa } \\
1\end{array}$ & 1.07 & 0.21 & 2.55E-38 & 4.77E-02 \\
\hline 131 & Q6IMZ3 & Annexin & Anxa6 & 2.81 & 0.29 & 2.95E-08 & $4.81 \mathrm{E}-02$ \\
\hline 132 & Q07009 & Calpain-2 catalytic subunit & Capn2 & 0.93 & 0.25 & $1.78 \mathrm{E}-06$ & $4.92 \mathrm{E}-02$ \\
\hline 133 & Q63362 & $\begin{array}{l}\text { NADH } \quad \text { dehydrogenase } \\
\text { [ubiquinone] } 1 \text { alpha subcomplex } \\
\text { subunit } 5\end{array}$ & Ndufa5 & 1.34 & 0.59 & 8.52E-04 & 4.93E-02 \\
\hline
\end{tabular}


Table 10 Gene family enriched proteins after PLN targeted APEX2 proximity labeling in NRCM 14-3-3 proteins

\begin{tabular}{|c|c|c|c|c|c|c|c|}
\hline \# & $\begin{array}{l}\text { Protein } \\
\text { IDs }\end{array}$ & Protein names & $\begin{array}{l}\text { Gene } \\
\text { names }\end{array}$ & $\begin{array}{c}\text { APEX2-PLN / } \\
\text { eGFP } \\
\text { Ratio } \\
\text { normalized } \\
\text { mean }\end{array}$ & \begin{tabular}{|} 
APEX2-PLN / \\
APEX2- \\
PLN $\Delta(1-29)$ \\
Ratio \\
normalized \\
mean
\end{tabular} & $\begin{array}{c}\text { APEX2- } \\
\text { PLN / } \\
\text { eGFP } \\
\text { z-test }\end{array}$ & $\begin{array}{l}\text { APEX2-PLN } \\
\text { APEX2- } \\
\text { PLN } \Delta(1-29) \\
\text { z-test }\end{array}$ \\
\hline 28 & P68255 & 14-3-3 protein theta & Ywhaq & 2.37 & 0.54 & 4.76E-30 & $1.68 \mathrm{E}-03$ \\
\hline 41 & P63102 & 14-3-3 protein zeta/delta & Ywhaz & 1.78 & 0.44 & 7.56E-36 & 3.76E-03 \\
\hline 49 & P68511 & 14-3-3 protein eta & Ywhah & 1.41 & 0.45 & $7.52 \mathrm{E}-11$ & 5.05E-03 \\
\hline 56 & P62260 & 14-3-3 protein epsilon & Ywhae & 1.81 & 0.40 & $1.29 \mathrm{E}-19$ & $5.90 \mathrm{E}-03$ \\
\hline 67 & P61983 & 14-3-3 protein gamma & Ywhag & 1.75 & 0.40 & $1.57 \mathrm{E}-13$ & 7.74E-03 \\
\hline 110 & Q5EBB0 & 14-3-3 sigma & Sfn & 2.33 & 0.65 & 1.16E-06 & $3.27 E-02$ \\
\hline
\end{tabular}

\section{Peroxiredoxins}

\begin{tabular}{|l|l|l|l|c|c|c|c|}
\hline$\#$ & $\begin{array}{l}\text { Protein } \\
\text { IDs }\end{array}$ & Protein names & Gene & $\begin{array}{c}\text { APEX2-PLN } / \\
\text { eGFP } \\
\text { Ratio } \\
\text { normalized } \\
\text { mean }\end{array}$ & $\begin{array}{c}\text { APEX2-PLN } / \\
\text { APEX2- } \\
\text { PLNA(1-29) } \\
\text { Ratio } \\
\text { normalized } \\
\text { mean }\end{array}$ & $\begin{array}{c}\text { APEX2- } \\
\text { PLN/ } \\
\text { eGFP } \\
\text { z-test }\end{array}$ & $\begin{array}{c}\text { APEX2-PLN } / \\
\text { APEX2- } \\
\text { PLNA(1-29) } \\
\text { z-test }\end{array}$ \\
\hline 31 & $\begin{array}{l}\text { A0A0G2K } \\
3 Z 9\end{array}$ & Peroxiredoxin-1 & Prdx1 & 2.31 & 0.31 & $1.35 \mathrm{E}-25$ & $2.04 \mathrm{E}-03$ \\
\hline 38 & Q9Z0V5 & Peroxiredoxin-4 & Prdx4 & 0.85 & 0.48 & $3.23 \mathrm{E}-04$ & $3.21 \mathrm{E}-03$ \\
\hline 63 & $\begin{array}{l}\text { A0A0G2JS } \\
\text { H9 }\end{array}$ & Peroxiredoxin-2 & Prdx2 & 2.61 & 0.23 & $1.67 \mathrm{E}-25$ & $6.80 \mathrm{E}-03$ \\
\hline 75 & $\begin{array}{l}\text { A0A0G2JS } \\
\text { S8 }\end{array}$ & Peroxiredoxin-5, mitochondrial & Prdx5 & 2.50 & 0.22 & $3.10 \mathrm{E}-11$ & $9.73 \mathrm{E}-03$ \\
\hline 78 & G3V7I0 & $\begin{array}{l}\text { Thioredoxin-dependent peroxide } \\
\text { reductase, mitochondrial }\end{array}$ & Prdx3 & 1.26 & 0.39 & $1.14 \mathrm{E}-07$ & $1.13 \mathrm{E}-02$ \\
\hline
\end{tabular}


LIM domain containing

\begin{tabular}{|l|l|l|l|c|c|c|c|}
\hline$\#$ & $\begin{array}{l}\text { Protein } \\
\text { IDs }\end{array}$ & Protein names & $\begin{array}{l}\text { Gene } \\
\text { names }\end{array}$ & $\begin{array}{c}\text { APEX2-PLN / } \\
\text { eGFP } \\
\text { Ratio } \\
\text { normalized } \\
\text { mean }\end{array}$ & $\begin{array}{c}\text { APEX2-PLN } / \\
\text { APEX2- } \\
\text { PLNA(1-29) } \\
\text { Ratio } \\
\text { normalized } \\
\text { mean }\end{array}$ & $\begin{array}{c}\text { APEX2- } \\
\text { PLN / } \\
\text { eGFP } \\
\text { z-test }\end{array}$ & $\begin{array}{c}\text { APEX2-PLN } / \\
\text { APEX2- } \\
\text { PLNA(1-29) } \\
\text { z-test }\end{array}$ \\
\hline 13 & G3V7U0 & $\begin{array}{l}\text { Cysteine and glycine-rich protein } \\
3\end{array}$ & Csrp3 & 2.34 & 0.40 & $4.22 \mathrm{E}-29$ & $2.30 \mathrm{E}-04$ \\
\hline 22 & P47875 & $\begin{array}{l}\text { Cysteine and glycine-rich protein } \\
1\end{array}$ & Csrp1 & 1.88 & 0.66 & $1.98 \mathrm{E}-03$ & $1.02 \mathrm{E}-03$ \\
\hline 37 & $\begin{array}{l}\text { A0A0G2K } \\
174\end{array}$ & LIM domain 7 & Lmo7 & 1.45 & 0.29 & $3.88 \mathrm{E}-03$ & $2.92 \mathrm{E}-03$ \\
\hline 54 & Q99MZ8 & LIM and SH3 domain protein 1 & Lasp1 & 1.43 & 0.67 & $4.56 \mathrm{E}-09$ & $5.70 \mathrm{E}-03$ \\
\hline 58 & P52944 & PDZ and LIM domain protein 1 & Pdlim1 & 2.13 & 0.45 & $3.25 \mathrm{E}-11$ & $6.05 \mathrm{E}-03$ \\
\hline 84 & Q6AYF2 & LIM and cysteine-rich domains 1 & Lmcd1 & 1.19 & 0.45 & $1.59 \mathrm{E}-14$ & $1.36 \mathrm{E}-02$ \\
\hline 88 & D4A7U1 & Zyxin & Zyx & 1.66 & 0.54 & $2.57 \mathrm{E}-23$ & $1.63 \mathrm{E}-02$ \\
\hline 119 & $\begin{array}{l}\text { A0A0G2JS } \\
\text { M3 }\end{array}$ & PDZ and LIM domain protein 3 & Pdlim3 & 2.51 & 0.36 & $7.04 \mathrm{E}-07$ & $3.92 \mathrm{E}-02$ \\
\hline
\end{tabular}

\section{Septins}

\begin{tabular}{|l|l|l|l|l|c|c|c|}
\hline$\#$ & $\begin{array}{l}\text { Protein } \\
\text { IDs }\end{array}$ & Protein names & Gene & $\begin{array}{c}\text { APEX2-PLN / } \\
\text { eGFP } \\
\text { Ratio } \\
\text { normalized } \\
\text { mean }\end{array}$ & $\begin{array}{c}\text { APEX2-PLN / } \\
\text { APEX2- } \\
\text { PLNA(1-29) } \\
\text { Ratio } \\
\text { normalized } \\
\text { mean }\end{array}$ & $\begin{array}{c}\text { APEX2- } \\
\text { PLN / } \\
\text { eGFP } \\
\text { z-test }\end{array}$ & $\begin{array}{c}\text { APEX2-PLN / } \\
\text { APEX2- } \\
\text { PLNA(1-29) } \\
\text { z-test }\end{array}$ \\
\hline 32 & B2GVB4 & Septin-9 & Sept9 & 1.2837 & 1.0102 & $2.93 \mathrm{E}-10$ & $2.20 \mathrm{E}-03$ \\
\hline 33 & F1LMC7 & Septin-7 & Sept7 & 2.4773 & 0.7412 & $5.90 \mathrm{E}-15$ & $2.27 \mathrm{E}-03$ \\
\hline 39 & $\begin{array}{l}\text { A0A0G2J } \\
\text { UL7 }\end{array}$ & Septin-11 & Sept11 & 0.9675 & 0.8788 & $3.92 \mathrm{E}-02$ & $3.53 \mathrm{E}-03$ \\
\hline 71 & Q91Y81 & Septin-2 & Sept2 & 1.4562 & 0.6876 & $1.81 \mathrm{E}-06$ & $8.94 \mathrm{E}-03$ \\
\hline
\end{tabular}


Troponin complex subunits

\begin{tabular}{|l|l|l|l|c|c|c|c|}
\hline$\#$ & $\begin{array}{l}\text { Protein } \\
\text { IDs }\end{array}$ & Protein names & Gene & $\begin{array}{c}\text { APEX2-PLN / } \\
\text { eGFP } \\
\text { Ratio } \\
\text { normalized } \\
\text { mean }\end{array}$ & $\begin{array}{c}\text { APEX2-PLN } / \\
\text { APEX2- } \\
\text { PLNA(1-29) } \\
\text { Ratio } \\
\text { normalized } \\
\text { mean }\end{array}$ & $\begin{array}{c}\text { APEX2- } \\
\text { PLN / } \\
\text { eGFP } \\
\text { z-test }\end{array}$ & $\begin{array}{c}\text { APEX2-PLN } / \\
\text { APEX2- } \\
\text { PLNA(1-29) } \\
\text { z-test }\end{array}$ \\
\hline 25 & P23693 & Troponin I, cardiac muscle & Tnni3 & 2.83 & 0.60 & $1.51 \mathrm{E}-28$ & $1.20 \mathrm{E}-03$ \\
\hline 59 & Q4PP99 & Cardiac troponin C & Tnnc1 & 1.82 & 0.30 & $8.99 \mathrm{E}-06$ & $6.19 \mathrm{E}-03$ \\
\hline 79 & A9YUA5 & Troponin T, cardiac muscle & Tnnt2 & 2.95 & 0.37 & $\begin{array}{c}7.90 \mathrm{E}- \\
105\end{array}$ & $1.21 \mathrm{E}-02$ \\
\hline 94 & D3ZZZ9 & $\begin{array}{l}\text { Catenin (Cadherin associated } \\
\text { protein), delta 1 (Predicted) }\end{array}$ & Ctnnd1 & 0.68 & 0.45 & $3.74 \mathrm{E}-02$ & $2.11 \mathrm{E}-02$ \\
\hline
\end{tabular}

\section{Small heat shock proteins}

\begin{tabular}{|l|l|l|l|c|c|c|c|}
\hline$\#$ & $\begin{array}{l}\text { Protein } \\
\text { IDs }\end{array}$ & Protein names & Gene & $\begin{array}{c}\text { APEX2-PLN / } \\
\text { eGFP } \\
\text { Ratio } \\
\text { normalized } \\
\text { mean }\end{array}$ & $\begin{array}{c}\text { APEX2-PLN / } \\
\text { APEX2- } \\
\text { PLNA(1-29) } \\
\text { Ratio } \\
\text { normalized } \\
\text { mean }\end{array}$ & $\begin{array}{c}\text { APEX2- } \\
\text { PLN / } \\
\text { eGFP } \\
\text { z-test }\end{array}$ & $\begin{array}{c}\text { APEX2-PLN / } \\
\text { APEX2- } \\
\text { PLNA(1-29) } \\
\text { z-test }\end{array}$ \\
\hline 9 & G3V913 & Heat shock protein beta-1 & Hspb1 & 1.28 & 0.22 & $6.10 \mathrm{E}-23$ & $2.97 \mathrm{E}-05$ \\
\hline 34 & P97541 & Heat shock protein beta-6 & Hspb6 & 1.25 & 0.47 & $1.90 \mathrm{E}-03$ & $2.45 \mathrm{E}-03$ \\
\hline 51 & 035878 & Heat shock protein beta-2 & Hspb2 & 2.17 & 0.22 & $2.28 \mathrm{E}-27$ & $5.31 \mathrm{E}-03$ \\
\hline
\end{tabular}

